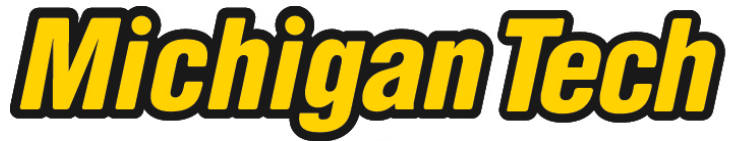 \\ Michigan Technological University Create the Future Digital Commons @ Michigan Tech
}

2014

\section{UTILIZING DIELECTROPHORESIS TO DETERMINE THE PHYSIOLOGICAL DIFFERENCES OF EUKARYOTIC CELLS}

Tayloria Nicole Gail Adams

Michigan Technological University

Follow this and additional works at: https://digitalcommons.mtu.edu/etds

Part of the Biomedical Engineering and Bioengineering Commons, and the Chemical Engineering Commons

Copyright 2014 Tayloria Nicole Gail Adams

\section{Recommended Citation}

Adams, Tayloria Nicole Gail, "UTILIZING DIELECTROPHORESIS TO DETERMINE THE PHYSIOLOGICAL DIFFERENCES OF EUKARYOTIC CELLS", Dissertation, Michigan Technological University, 2014.

https://doi.org/10.37099/mtu.dc.etds/855

Follow this and additional works at: https://digitalcommons.mtu.edu/etds

Part of the Biomedical Engineering and Bioengineering Commons, and the Chemical Engineering Commons 


\title{
UTILIZING DIELECTROPHORESIS TO DETERMINE THE PHYSIOLOGICAL DIFFERENCES OF EUKARYOTIC CELLS
}

By

Tayloria Nicole Gail Adams

\begin{abstract}
A DISSERTATION
Submitted in partial fulfillment of the requirements for the degree of DOCTOR OF PHILOSOPHY

In Chemical Engineering
\end{abstract}

MICHIGAN TECHNOLOGICAL UNIVERSITY

2014

(C) 2014 Tayloria N.G. Adams 
This dissertation has been approved in partial fulfillment of the requirements for the Degree of DOCTOR OF PHILOSOPHY in Chemical Engineering.

Department of Chemical Engineering

Dissertation Advisor: Dr. Adrienne Minerick

Committee Member: Dr. Caryn Heldt

Committee Member: Dr. Feng Zhao

Committee Member: $\quad$ Dr. Chang K. Choi

Department Chair: $\quad$ Dr. S. Komar Kawatra 
In loving memory of Jonah Adams Jr.

A father is respected because he gives his children leadership.

Appreciated because he gives his children care.

Valued because he gives his children time.

Loved because he gives his children the one thing they treasure most himself.

--Anonymous 


\section{TABLE OF CONTENTS}

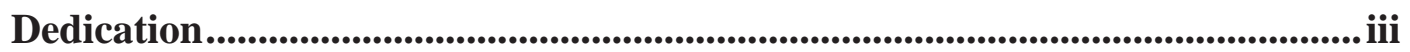

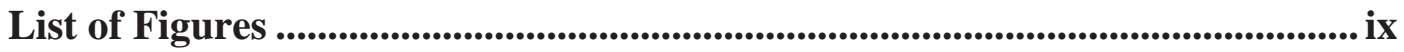

List of Tables ........................................................................................................ xvii

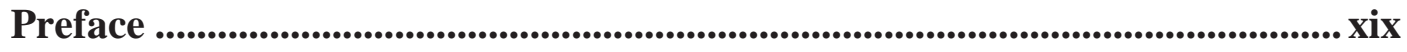

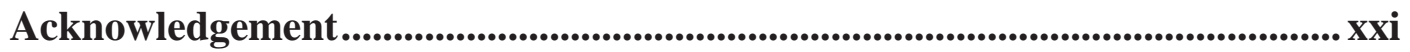

List of Publications ................................................................................................. xxiii

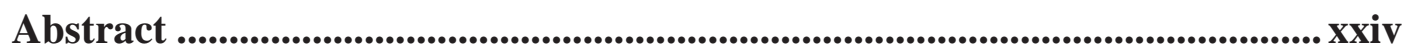

Chapter 1: Introduction ...................................................................................... 1

1.1 Motivation: Stem Cell Therapy and Diabetes Mellitus ................................. 1

1.2 Successful hMSC Therapies and Insulin Producing MSCs............................ 5

1.3 Dielectrophoresis and Electrokinetics .................................................... 8

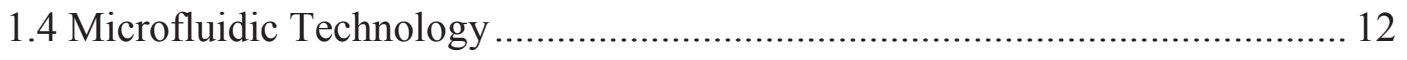

1.5 Dielectrophoretic Microfluidic Devices and Diabetes Treatment ................... 13

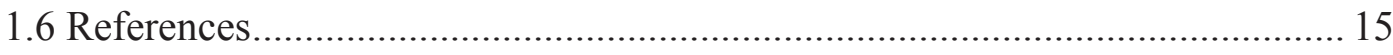


2.1 Key Properties of Human Mesenchymal Stem Cells ..................................... 24

2.1.1 hMSC Self-Renewal, Differentiation Potential and Trophic Activity........ 25

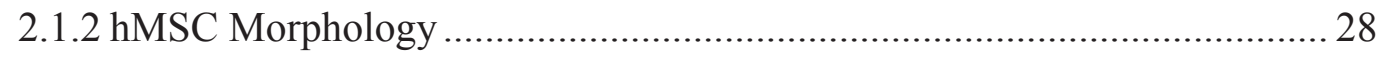

2.1.3 Elastin-Like Polypeptide Polyethyleneimine........................................... 29

2.1.4 hMSCs Surface Biosurface Markers........................................................ 29

2.1.5 hMSCs Mechanical Properties............................................................ 33

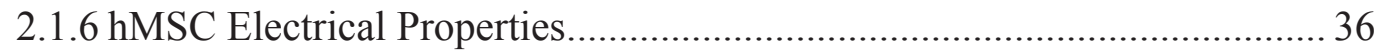

2.2 Human Mesenchymal Stem Cells Separation Technology ............................ 38

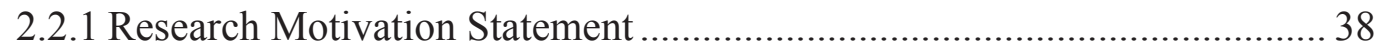

2.2.2 Fluorescent and Magnetic Activated Cell Sorting for Cell Separation....... 39

2.2.3 Density Centrifugation and Membrane Filtration................................... 44

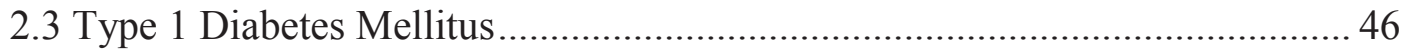

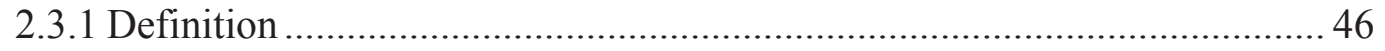

2.3.2 hMSCs as a Type 1 Diabetes Treatment Option.................................... 50

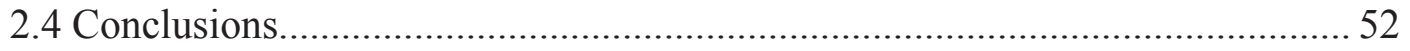

2.4.1 Dielectrophoresis for hMSC Separation ........................................... 53

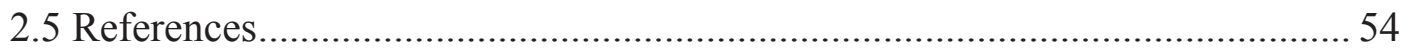

Chapter 3: Dielectrophoresis Literature Review................................................... 72

3.1 Brief Overview: hMSC Separation Shortcomings and Research Objectives ... 72 


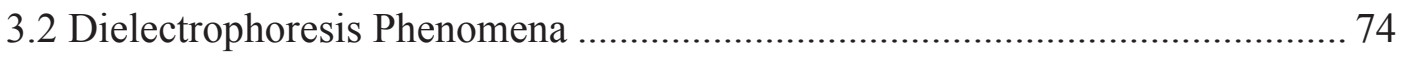

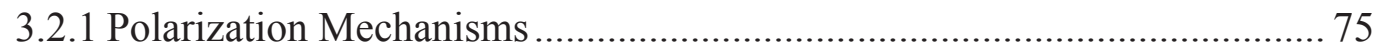

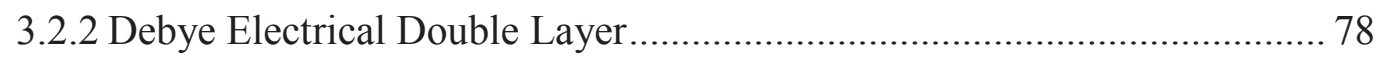

3.3 Dielectrophoretic Particle Polarization Model ................................................ 79

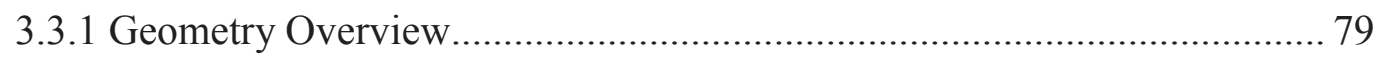

3.3.2 Homogeneous Sphere DEP Polarization Model.......................................... 79

3.3.3 Core-Shell Spherical DEP Polarization Model........................................... 83

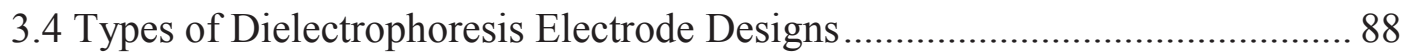

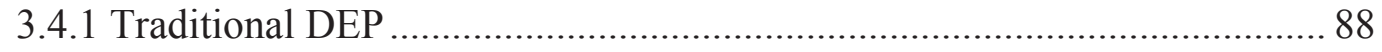

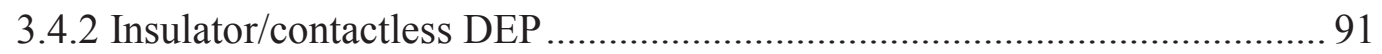

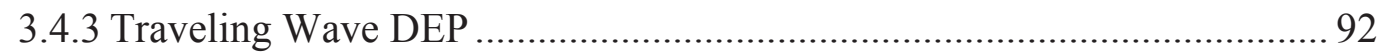

3.5 Microdevice Dielectrophoretic Applications................................................. 95

3.5.1 General Applications and Stem Cell Studies............................................. 95

3.5.2 DEP Studies to Determine Cell Membrane Capacitance ……….............. 100

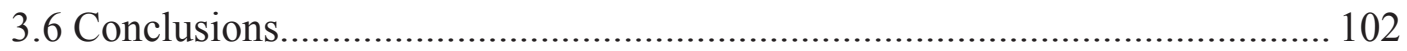

Chapter 4: Characterizing the Dielectric Properties of Human Mesenchymal Stem Cells and the Effects of Charged Elastin-like Polypeptide Copolymer

Treatment ........................................................................................................................... 112

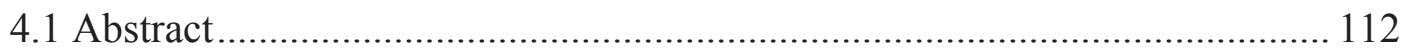

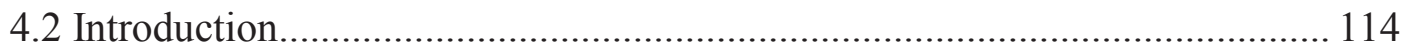

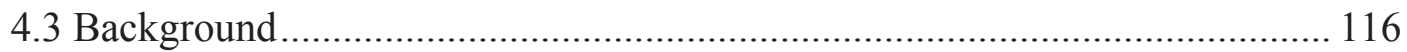


4.4 Materials and Methods

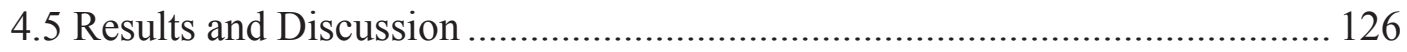

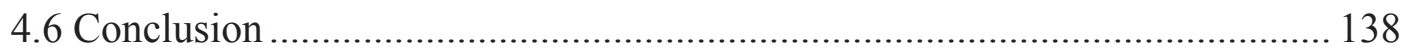

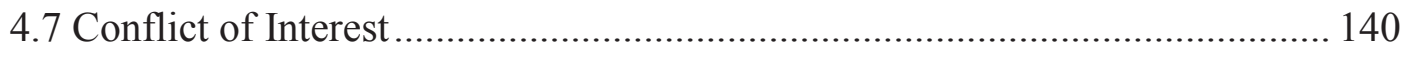

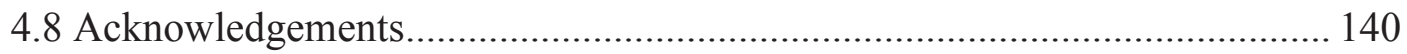

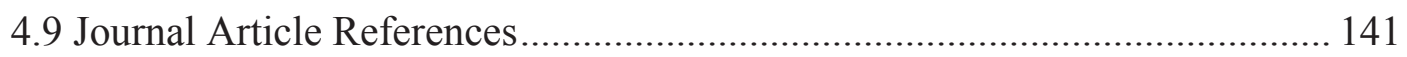

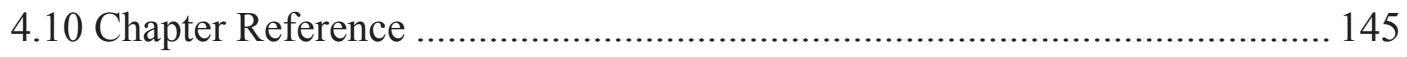

Chapter 5: Frequency Sweep Rate Dependence on the Dielectrophoretic Response of Polystyrene Beads and Red Blood Cells ............................................ 146

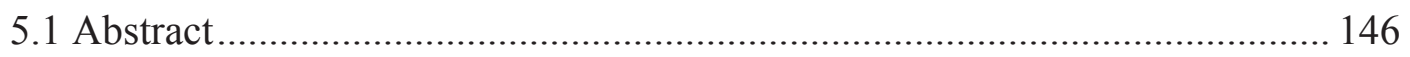

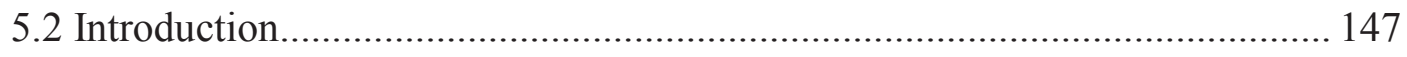

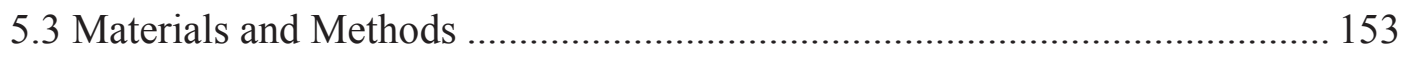

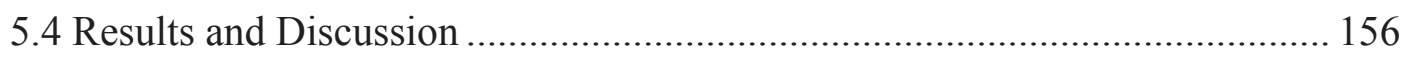

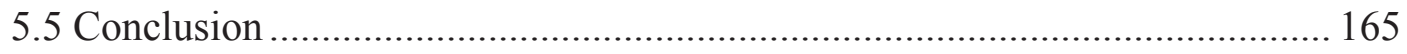

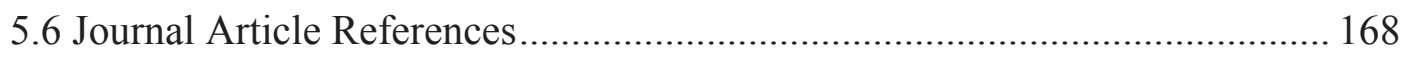

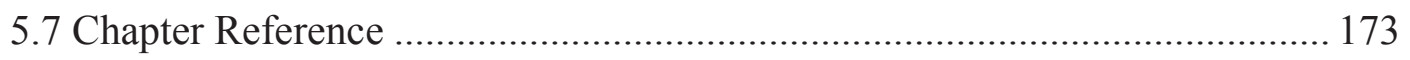


Chapter 6 Conclusions: Knowledge Gained from the Dielectrophoretic Study of hMSCs for Type 1 Diabetes .......................................................................... 174

6.1 Summary of Human Mesenchymal Stem Cells, Type 1 Diabetes, and Dielectrophoretic Background Knowledge .................................................... 174

6.1.1 hMSC Dielectrophoretic Characterization - Objective 1 ....................... 176

6.1.2 hMSC Dielectric Properties Modeled - Objective 2 ............................. 178

6.1.3 Frequency Sweep Rate Dielectrophoretic Experimental Method ............ 181

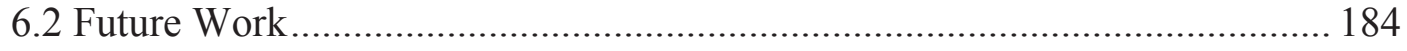

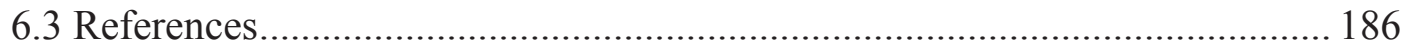

Appendix A: A Tunable Microfluidic Device for Drug Delivery ........................... 193

Appendix B: Copyright Permission Documentation................................................ 243 


\section{List of Figures}

Figure 1.1: Flow diagrams outlining the steps necessary to achieve hMSC isolation using (a) density gradient centrifugation with FACS or MACs and (b) the presently

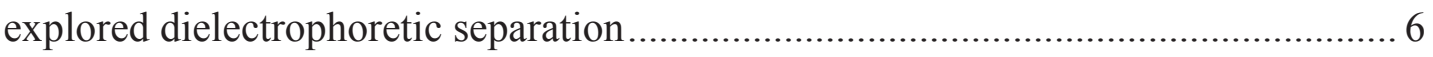

Figure 1.2: Nonuniform dielectrophoretic particle polarization ............................ 10

Figure 2.1: Important properties of hMSCs (a) trophic activity, (b) self-renewal, and (c) differentiation potential $[2,18,23]$ 28

Figure 2.2: hMSCs morphological subpopulations (a) rapidly self-renewing, star-like shape, (b) elongated fibroblastic-like spindle shaped cells, and (c) slowly-replicating flattened cells [26]

Figure 2.3: Illustration of differentiation surface proteins present within one hMSC population $[33,34]$ 32

Figure 2.4: Impedance measurements for (a) undifferentiated hMSCs (red) and osteocyte differentiated hMSCs (blue) (yellow is a nonrelated mikrozoid liquid 
treatment to induce cell necrosis), and (b) undifferentiated hMSCs (labeled control) and adipocyte differentiated hMSCs (labeled differentiation) $[55,56]$ 37

Figure 2.5: (a) Schematic of Fluorescent-Activated Cell Sorting and (b) illustrates labeling of two hMSCs progenitor cells [64-65]

Figure 2.6: (a) Schematic of Magnetic-Activated Cell Sorting and (b) cell labeled with magnetic beads, (c) unlabeled cell [74-76].

Figure 2.7: (a) Schematic of density gradient centrifugation [86] and, (b) electron micrograph of nonwoven rayon and polyethylene with hMSCs attached [87] 45

Figure 2.8: Schematic of Type 1 Diabetes (a) normal functioning pancreas and (b) diminished pancreas function, beta cells destruction $[89,90]$.

Figure 3.1: Schematic of negative DEP and positive DEP a cell will experience in an

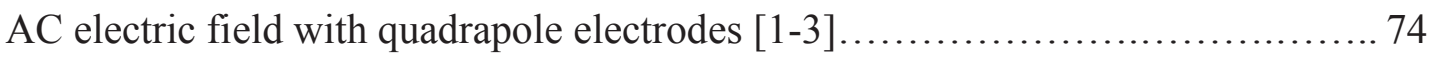

Figure 3.2: Force exerted upon a small dipole by an electric field [4]. 77 
Figure 3.3: Homogeneous sphere DEP polarization models for the $f_{C M}$ of hMSCs in

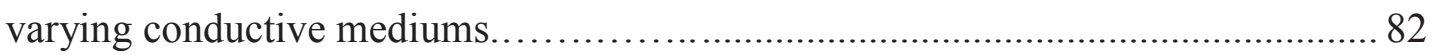

Figure 3.4: Schematic of core-shell spherical cell and equivalent sphere with the coreshell spherical DEP polarization model for hMSCs $f_{C M}$ in varied conductive

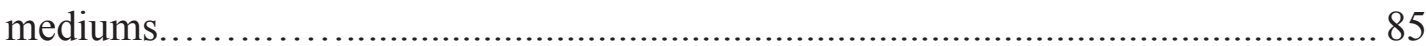

Figure 3.5: Effect of membrane size difference on hMSCs $f_{C M}$ directly correlates to

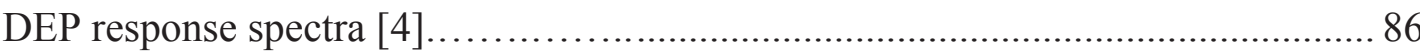

Figure 3.6: Common traditional DEP electrode designs (a) interdigitated, (b) castellated, (c) curved, (d) quadrapole. Each device design is used for cell trapping based on cell dielectric properties [12] 89

Figure 3.7: Varied insulating posts design inside iDEP microdevices. (a) rounded triangluar [27], (b) circular [33], (c) elongated [32], and (d) triangular [30].............. 
Figure 3.8: twDEP separation of jurkat cells from S. cervisiae and Lactobacillus casei bacteria. Jurkat cells were separated by flowing along the twDEP field gradient with $\mathrm{nDEP}$ force and the bacteria cells were adhered to the electrodes with pDEP force 94

Figure 3.9: Shows the cell sorting microdevice and corresponding SEM images of

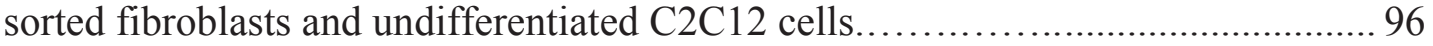

Figure 3.10: Real image of the parallel electrode microfluidic device used to examine

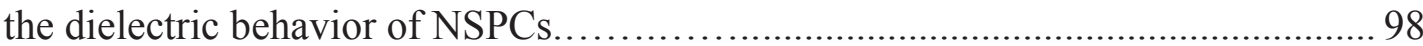

Figure 3.11: Percent trappings results for NSPCs, neurons, and astrocytes. Each cell type has distinct percentage of cells trapped at particular frequencies, which is an indication of unique dielectric properties among each cell type.....................99

Figure 4.1: (a) hMSCs cartooned to emphasize the differing cell membrane biosurface markers and thus properties. After ELP-PEI treatment, cartooned spheroidal morphology. (b) Quadrapole microdevice with $200 \mu \mathrm{m}$ spaced Ti-Au electrodes used for DEP experiments. (c) hMSCs in device with field off, and the DEP response observed with field on. At a single frequency, both $\mathrm{nDEP}$ and $\mathrm{pDEP}$ were observed 
due to differing membrane biosurface markers within an hMSCs

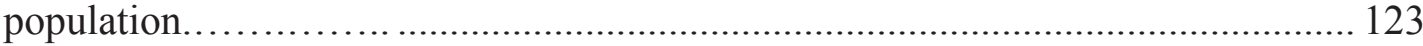

Figure 4.2: (a) Microscope images of untreated hMSCs DEP response with the field off and at $0.010,1.0$, and $10 \mathrm{MHz}$ after $90 \mathrm{~s}$ in $0.10 \mathrm{~S} / \mathrm{m}$ dextrose solution. At 0.010MHz, hMSCs only display nDEP and display both nDEP and pDEP at $10 \mathrm{MHz}$. (b) Table of total cells for each image. (c) DEP responses tabulated as $\%$ cell response $\left(R_{c}\right)$ into a stacked column chart. nDEP dominates at $0.010 \mathrm{MHz}$ and at $10 \mathrm{MHz} \mathrm{pDEP}$ is $79 \%$ dominant.

Figure 4.3: (a) Percent cell response $R_{c}$ for untreated hMSCs in $0.030 \mathrm{~S} / \mathrm{m}$ dextrose solution. (b) $R_{c}$ for untreated hMSCs in $0.10 \mathrm{~S} / \mathrm{m}$ dextrose solution. For both conductivities, nDEP dominates lower frequencies while pDEP dominates higher frequencies. (c) Data from a and b translated into DEP response spectra for untreated hMSCs at $0.030 \mathrm{~S} / \mathrm{m}$ and $0.10 \mathrm{~S} / \mathrm{m}$. This format best illustrates $0.030 \mathrm{~S} / \mathrm{m} f_{x o}$ estimated as $0.62 \mathrm{MHz}$ and $0.10 \mathrm{~S} / \mathrm{m} f_{x o}$ estimated as $1.3 \mathrm{MHz}$

Figure 4.4: (a) Untreated hMSC DEP responses at 0.030 and $0.10 \mathrm{~S} / \mathrm{m}$ compared to the core-shell spherical model. (b) hMSC membrane capacitance and (c) membrane permittivity based on experimentally determined cross-over frequency........... 130 
Figure 4.5: Untreated hMSCs (first row) compared to ELP-PEI treated hMSC spheroidal morphology (second row), both in $0.10 \mathrm{~S} / \mathrm{m}$ dextrose solution. (a) untreated hMSCs in cell culture flask, (b) treated hMSCs after $24 \mathrm{hrs}$ in cell culture flask, (c) untreated and (d) treated hMSCs in microdevice with field off where black regions are the quadrapole electrodes. Untreated and treated hMSCs at $10 \mathrm{Vpp}$ and $0.010 \mathrm{MHz}(\mathrm{e}$ and f), 1.0MHz (g and h), and 10MHz (i and j). (k) DEP response spectra of untreated and ELP-PEI treated hMSCs in $0.10 \mathrm{~S} / \mathrm{m}$. Untreated cells transition from $\mathrm{nDEP}$ to pDEP at $\sim 1.3 \mathrm{MHz}$, while treated cells predominantly exhibit nDEP behaviors and formed spheroidal aggregates. 132

Figure 4.6: Key parameter optimizations for the core-shell spherical DEP polarization model for ELP-PEI treated hMSCs at $0.10 \mathrm{~S} / \mathrm{m}$. (a) Decreases from literature values in membrane permittivity better capture ELP-PEI treated hMSCs nDEP behaviors. (b) Increases or decreases in membrane conductivity do not contribute to the experimentally observed nDEP. (c) Decreases in cytoplasm permittivity also do not contribute. (d) Decreases in cytoplasm conductivity may contribute to the nDEP behavior with $\sigma_{\text {cyto }}=6.3 \times 10^{-3} \mathrm{~S} / \mathrm{m}$ fitting well. (e) Model prediction for both untreated and treated hMSCs at $0.10 \mathrm{~S} / \mathrm{m}$ using all optimal parameters for each. Agreement between the model and the data is quite good................................................ 134 
Figure 5.1: (a) Dielectric relaxation mechanism for PS beads showing cases when i) particle polarization occurs at a static frequency, ii) $\tau_{\mathrm{MW}}$ is shorter than the slow frequency sweep rate $\left(\tau_{\Delta \mathrm{FS}}\right)$ allowing the bead interface time to polarize in response to the nonuniform $\mathrm{AC}$ field, and iii) $\tau_{\mathrm{MW}}$ is longer than the $\tau_{\Delta \mathrm{FS}}$ for fast frequency sweep rates and the bead interface does not have time to fully polarize. (b) Schematic of the quadrapole electrodes micro patterned onto a glass slide, and (c) microdevice with PDMS fluidic layer bonded above the quadrapole electrodes silver epoxies to copper leads. 152

Figure 5.2: (a) nDEP behavior of $6 \mu \mathrm{m}$ PS beads suspended in E-pure $\mathrm{H}_{2} \mathrm{O} 2.5 \times 10^{-4}$ $\mathrm{S} / \mathrm{m}$ and $250 \mathrm{~V}_{\mathrm{pp}} / \mathrm{cm} 0.0063,0.056$ and $0.17 \mathrm{MHz} / \mathrm{s}$ sweep rates from $0.010 \mathrm{MHz}$ to 1.0 MHz. (b) Raw intensity profile of PS beads in the center $\mathrm{nDEP}$ region (boxes shown at $0.20 \mathrm{MHz}$ ) at $0.0063 \mathrm{MHz} / \mathrm{s}$ sweep rate. Inset is a calibration of intensity per bead. (c) Clausius-Mossotti factor for the PS beads from $0.010 \mathrm{MHz}$ to $2.0 \mathrm{MHz}$ at three conductivities of $2.5 \times 10^{-4}, 1.0 \times 10^{-3}$, and $1.0 \mathrm{~S} / \mathrm{m}$. PS bead assembly at slower frequency sweep rates track static frequency responses while $0.056 \mathrm{MHz} / \mathrm{s}$ illustrates transitional behavior and frequency sweeps above $0.17 \mathrm{MHz} / \mathrm{s}$ substantially lag the true static frequency DEP responses....................................................... 157

Figure 5.3: (a) $6 \mu \mathrm{m}$ PS beads nDEP intensity profiles for $0.00080,0.0063$, and 0.056 $\mathrm{MHz} / \mathrm{s}$ and static steady state (SS) measurements (black diamonds). Intensity analysis 
captures bead assembly to the quadrapole center with transient and SS regions. The slowest frequency sweep rate of $0.00080 \mathrm{MHz} / \mathrm{s}$ best predicts the static DEP responses. (b) Bead assembly profiles for $0.0063(\mathrm{n}=8)$ and $0.17 \mathrm{MHz} / \mathrm{s}(\mathrm{n}=7)$ with $95 \%$ confidence upper and lower limits shown as dashed lines. (c) Transient slope comparison for static frequencies $(0 \mathrm{MHz} / \mathrm{s})$ as well as frequency sweeps. (d) Comparison of static frequency and frequency sweep PS bead velocities from 0 to 50s. $0.00080 \mathrm{MHz} / \mathrm{s}$ results are consistently similar to the static frequency results.

Figure 5.4: (a) nDEP behavior of RBCs suspended in $0.1 \mathrm{~S} / \mathrm{m}$ dextrose buffer and $250 \mathrm{~V} / \mathrm{cm}$ at $0.00080 \mathrm{MHz} / \mathrm{s}, 0.0063 \mathrm{MHz} / \mathrm{s}$ and $0.056 \mathrm{MHz} / \mathrm{s}$ sweep rates from 0.010 $\mathrm{MHz}$ to $0.50 \mathrm{MHz}$. (b) RBCs nDEP intensity profiles for $0.00080,0.0063$, and 0.056 $\mathrm{MHz} / \mathrm{s}$ and static measurements. (c) 0.00080 and $0.056 \mathrm{MHz} / \mathrm{s} \mathrm{RBC}$ assembly profiles $\mathrm{n}=8$, with $95 \%$ confidence interval upper and lower limits shown as dashed

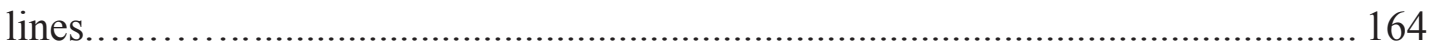




\section{List of Tables}

Table 2.1: Summary of the hMSCs immunophenotyping [15, 30-33]....

31

Table 2.2: Elasticity and Viscoelasticity of hMSCs with spherical and spread star-like morphologies [35] 34

Table 3.1: High and low frequency examination of the Clausius-Mossotti factor, $f_{C M}$

Table 3.2: Summary of electrode configuration applications

90

Table 3.3: Summary of cell membrane capacitance studies using DEP. 101

Table 4.1: Summary of literature and optimized parameters for core-shell spherical DEP polarization model for untreated and treated $\mathrm{hMSCs}$ in $0.030 \mathrm{~S} / \mathrm{m}$ and $0.10 \mathrm{~S} / \mathrm{m}$

Table 6.1: Cell membrane capacitance comparison between hMSCs and other cell systems. Neural stem/progenitor cells (NSPCSs) membrane capacitance was xvii 
characterized based on its progenitor cells neurogenic progenitor (NP) and astrogenic progenitor (AP) [52]. Human mesenchymal stem cells (hMSCs) membrane capacitance was characterized by work completed in this dissertation [53]. CaLH3, H357, OSCC1, DOK are oral squamous cell carcinomas characterized into three subpopulations based on cell adhesion: rapid adherent cells (RAC), middle adherent cells (MAC) and late adherent cells (LAC), which correlated to the cells tumorigenic capabilities [47]. Adipose-derived stem cells membrane capacitance was characterized based on subpopulations determined by differentiation (osteoblasts), and progenitor cells (adipogenic and osteogenic) [48] 180 


\section{Preface}

The subject of this dissertation is dielectrophoresis and human mesenchymal stem cells (hMSCs) for cell separation and type 1 diabetes treatment. Dielectrophoresis is a unique characterization technology with a variety of utilities explicitly identified within the dissertation. hMSCs are a specialized source of cells readily acquired from bone-marrow, and have been explored as a therapeutic option for type 1 diabetes (research motivation).

The aim of this work is to provide sufficient background information on hMSCs to illustrate their utility in the medical field, introduce DEP as an advantageous separation technique to purify hMSCs after extraction from bone-marrow, and define a new DEP data collection technique.

Many individuals have made valuable contributions to the preparations of the chapters found within this dissertation. I would like to give a special thanks to my advisor Dr. Adrienne Minerick for all of her constructive feedback and editing of each chapter in this dissertation. She provided critical feedback with data analysis and interpretation for Chapters 4 and 5, and she is co-author on the papers published in Chapters 4 and 5. I would like to graciously thank Dr. Feng Zhao for providing me with the human mesenchymal stem cells used to collect all data in Chapter 4, and for her co-authorship of Chapter 4. I would like to thank my collaborators from the University of Mississippi Medical Center, Dr. Amol Janorkar and Paul Turner for providing me with generous amounts of elastin-like polypeptide polyethyleneimine 
copolymer used to collect data for the human mesenchymal stem cell treatments. I would also like to thank them for their review, data interpretation, and co-authorship on Chapter 4. I would also like to thank Dr. Kaela Leonard for her assistance with Chapter 5. She collected and analyzed the data for the red blood cell DEP response as well as, reviewed and co-authored Chapter 5.

I would like to thank Chungja Yang, John Gress, and Nick Wimmer, and Dr. Adrienne Minerick for their contributions to the book chapter A Tunable Microfluidic

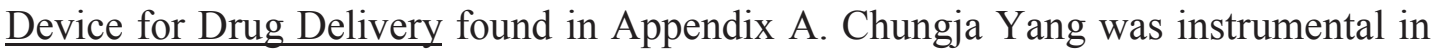
the COMSOL simulations presented throughout the chapter. She and I co-authored the book chapter equally. I would like to thank Nick Wimmer for the concept of using microneedles as a drug delivery option and preparing the initial writings on this topic. And I would like to thank John Gress, for his idea on a feedback loop as a method to monitor the concentration of drugs delivered. I would like to thank Dr. Adrienne Minerick for her guidance and assistance with the development of our drug delivery idea. Her insight, excitement and recognizing the potential in our idea is what led to it be published as a book chapter. Dr. Minerick helped write and edit the book chapter. Each person mentioned was instrumental in preparing Chapter 4, 5, and Appendix A as manuscripts and a book chapter. I wrote and edited Chapters 1-6 in this dissertation, and I am first author on the publications in Chapters 4 and 5. I collected and analyzed all of the human mesenchymal stem cell data in Chapter 4, and collected and analyzed the polystyrene bead data in Chapter 5. I share equal co-authorship with Chungja Yang on the book chapter found in Appendix A. 


\section{ACKNOWLEDGEMENTS}

First and foremost I would like to thank God for blessing me to reach this point

in my career. For all things are possible through Christ who strengthens me. I would like to thank my advisor Dr. Adrienne Minerick along with my committee members Dr. Caryn Heldt, Dr. Feng Zhao, and Dr. Chang Choi. Your knowledge, insight, and guidance throughout my research and graduate school career has been very helpful.

I would like to thank the King-Chavez-Parks State of Michigan Fellowship, MTU's Doctoral Finishing Fellowship, MTU Graduate School Dean's Fellowship, and the Anderson Scholarship for providing funding for this work.

I would like to thank my friends Carnell Hunter, Renee Oats, Kabongo Ngandu, Ashli Fueri, Gabriel Agbrouche, Sheraaron Hurt, Juanika Dildy, and Priscilla Griffin. Your advice and encouragement over the years has been irreplaceable. I would like to thank my research colleagues and friends Chungja Yang, Ran An, Zhichao Wang, Hwi Yong Lee, Dr. Kaela Leonard, Hongyu Xie, Jeana Dillion, and Maryam Khaksari. Your research advice and jokes have really made my time at MTU enjoyable. I can't wait to see what the future has in store for all of us!

I would like to thank the Center for Diversity and Inclusion (CDI), Shezwae Fleming, Kellie Raffaelli, Renee Wells, Karen Wade, Robert Richards, and Amy Mensch, your dedication to helping graduate students has been wonderful. CDI became my Michigan Tech family, I will miss you all! 
My family holds a special place in my heart; I would like to thank my sisters Dawn Adams and Monica Sprowls, you both have been a great support system. I would like to thank my brothers Jonah Adams III and Brandon Sprowls; whenever I was awake past 3am I knew I could call or text my little brother for great conversation, thanks Jonah! I would like to thank my mother, Gloria Adams, you're my biggest cheerleader and you have always encouraged me to strive, work hard, and do more. I love you with all of my heart. I would like to thank my grandmother, Linda Adams, for being a solid rock for me and always knowing what to say when I called. We are namesakes and I will always cherish that. And to my father, who passed away far too soon, thank you for providing me with the opportunities to reach this moment in my life. Gone 11 years, but you are always close to me.

Finally, I would like to give the biggest thank you to my daughter, Aiyanna Adams. She has been my biggest inspiration. I love you! 


\section{List of Publications}

(1) Adams, T.N.G., Turner, P., Zhao, F., Janorkar, A., and Minerick, A.R., Characterizing the Dielectric Properties of Human Mesenchymal Stem Cells and the Effects of Elastin-like Polypeptide Polyethyleneimine Polymer Treatment, Biomicrofluidics, 8, 054109, 2014.

(2) Adams, T.N.G., Leonard, K.M., and Minerick, A.R., Frequency Sweep Rate Dependence on the Dielectrophoretic Response of Polystyrene Beads and Red Blood Cells, Biomicrofluidics, 7, 064114, 2013.

(3) Minerick, A., Collins, J., Leonard, K., and Adams, T.N.G., Methods and Systems for Identifying a Particle Using Dielectrophoresis, International Patent Application No. PCT/US2014/059332, filed October 2014.

(4) Adams, T., Yang, C., Gress, J., Wimmer, N., and Minerick, A.R., A Tunable Microfluidic Device for Drug Delivery, Advances in Microfluidics, InTech Open Science, 2012.

(5) Adams, T.N.G., Olson, T., King, J., and Keith, J., In-Plane Thermal Conductivity Modeling of Carbon Filled Liquid Crystal Polymer Based Resins, Journal of Polymer Composites, 32, 147-157, 2011. 


\begin{abstract}
Type 1 diabetes affects over 108,000 children, and this number is steadily increasing. Current insulin therapies help manage the disease but are not a cure. Over a child's lifetime they can develop kidney disease, blindness, cardiovascular disease and many other issues due to the complications of type 1 diabetes. This autoimmune disease destroys beta cells located in the pancreas, which are used to regulate glucose levels in the body. Because there is no cure and many children are affected by the disease there is a need for alternative therapeutic options that can lead to a cure.
\end{abstract}

Human mesenchymal stem cells (hMSCs) are an important cell source for stem cell therapeutics due to their differentiation capacity, self-renewal, and trophic activity. hMSCs are readily available in the bone marrow, and act as an internal repair system within the body, and they have been shown to differentiate into insulin producing cells. However, after isolation hMSCs are a heterogeneous cell population, which requires secondary processing. To resolve the heterogeneity issue hMSCs are separated using fluorescent- and magnetic-activate cell sorting with antigen labeling. This techniques are efficient but reduce cell viability after separation due to the cell labeling. Therefore, to make hMSCs more readily available for type 1 diabetes therapeutics, they should be separated without diminishing there functional capabilities. Dielectrophoresis is an alternative separation technique that has the capability to separated hMSCs.

xxiv 
This dissertation uses dielectrophoresis to characterize the dielectric properties of hMSCs. The goal is to use hMSCs dielectric signature as a separation criteria rather than the antigen labeling implemented with FACS and MACS. DEP has been used to characterize other cell systems, and is a viable separation technique for hMSCs. 


\section{Chapter 1 Introduction}

Stem cell therapy has gained popularity in medical research especially as a therapeutic option for chronic disease treatment. This chapter will overview the main topic of this dissertation, which is utilizing dielectrophoretic technology to purify human mesenchymal stem cells for type 1 diabetes treatment. The sections to follow will motivate the work with statistics on the prevalence of type 1 diabetes in the U.S. and world, briefly describe human mesenchymal stem cells differentiation toward insulin producing cells, dielectrophoresis, microfluidics, and the combination of the technologies.

\subsection{Motivation: Stem Cell Therapy and Diabetes Mellitus}

Heart disease, stroke, and diabetes mellitus are chronic diseases that 27.3 million Americans (or $8.7 \%$ U.S. population) live with and these diseases claim 800,000 lives each year [1-4]. Many chronic diseases are treatable by diet and exercise $[5,6]$ while others like type 1 diabetes $(0.8 \%$ of all diabetes mellitus cases $)$ require therapeutic options [7]. Type 1 diabetes, mostly diagnosed in children, is a result of the pancreas not producing insulin and thus requiring insulin therapeutics [8]. This means that young children, through their entire life, inject themselves with insulin multiple times a day, making the disease difficult to manage. More than 108,000 children between 0 -14 years have type 1 diabetes with 16,000 new cases diagnosed each year [9]. In 2007, it cost $\sim \$ 116$ billion to treat diabetes, and as children become adults complications include heart disease, stroke, blindness, kidney 
disease, hypertension, amputations, dental disease, and pregnancy complications [10]. Insulin therapy is in place as a management tool, but does not solve the main issue of beta cell destruction in type 1 diabetes. This necessitates the development of treatment options that can potentially cure type 1 diabetes rather than just intermittently managing it. The International Diabetes Federations tracks the shows the prevalence of diabetes in adults and children worldwide. For the western hemisphere, diabetes in most prevalent in Mexico with $>12 \%$ of the population affected. The U.S. and Brazil come in second with 9-12\% of the population affected. It is projected that 592 million people will suffer from diabetes in 2035 compared to 382 million people in 2013 [9], (see http://www.idf.org/diabetesatlas ).

Human mesenchymal stem cells (hMSCs) are an interesting cell source to researchers because of their regenerative $[11,12]$ and immunological properties [13]. hMSCs are exciting in medical research because they can serve as an internal repair system in the body. Upon injury, signals are sent to hMSCs and they migrate to those specific injured areas, differentiate, and promote healing [12, 14]. hMSCs are unspecialized cells isolated primarily from bone-marrow [15] demonstrating significant properties such as high differentiation capacity (adipocytes, chondrocytes, osteoblasts, etc.) $[14,16,17]$, self-renew [18, 19], and secretion of bioactive molecules (trophic activity) $[12,13,20,21]$. Trophic activity is significant to hMSCs function because signals are sent to surrounding cells and trigger them to perform specific roles such as tissue regeneration $[20,21]$. 
One problem associated with hMSC bone-marrow isolation is that they are obtained as a heterogeneous mixture $[22,23]$. In order for hMSCs to be used as an effective therapeutic, they must first be purified. For therapeutic treatments, obtaining hMSCs is a multistep process. Following bone-marrow isolation, hMSCs are centrifuged via density gradient solution (step 1), adhered to a plastic cell culture dish (step 2), and separated using trypsinization (step 3) [24]. This method is inefficient and time consuming taking days to complete [25], so other separation techniques have been employed. Fluorescent- and magnetic-activated cell sorting (FACS and MACS) use unique cell-surface recognition elements to tag target cells. This cell labeling alters cellular function, which is not desirable in therapeutics. Cell culture purification, FACS, and MACS approaches all require expensive raw materials and are labor intensive [25-27].

Other drawbacks with hMSCs are that their natural inherent biological functions are not well understood, and within one population hMSCs, cells express different membrane surface proteins [28]. Currently there is not one unique marker (surface proteins, differentiation pathway, plasticity in cell culture, etc.) that distinguishes hMSCs from other cell populations [22, 29] or identify their disposition to differentiate to specific cell types. The minimum requirements established by the International Society for Cellular Therapy to name a cell population hMSCs are: (1) plastic adherence (generic cellular property [14]); (2) positive expression of biosurface markers CD105, CD73, and CD90; (3) negative expression for CD34, 
CD45, CD11a, CD19, CD14, and HLA-DR; and (4) adipogenic, osteogenic, and chondrogenic differential potential $[17,30]$.

Additionally, hMSCs morphology varies creating subpopulations [31], which adds another dimension to overcome for effective purification. From this point forward, purification and separation terminology will be used interchangeably. According to Haasters et al., hMSCs can be assigned three morphological subpopulations: (1) rapidly self-renewing cells, triangular or star-like shape; (2) elongated, fibroblastic-like, spindle-shaped cells; and (3) slowly-replicating, large cuboidal or flattened cells [31]. These subpopulations are dependent on cytoskeleton, cell adhesion, and active pathways that directly determine cell fate and differentiation [31]. Others have looked at hMSCs viscoelastic properties, the tendency to exhibit viscous and elastic characteristics under deformation, because it plays an important role in a cell's biological response [32]. In the study completed by Darling et al., the elasticity and viscoelasticity of two distinct spherical and spread star-like hMSC morphologies were examined. The elasticity was found to be $2.5 \mathrm{kPa}$ for spherical and $3.2 \mathrm{kPa}$ for spread star-like hMSCs, while the viscoelasticity of the spherical morphology was $0.47 \mathrm{kPa}$ the spread star-like was $2.2 \mathrm{kPa}$. These results show how hMSC morphology changes subpopulation properties.

Elastin-like polypeptide (ELP) polyethyleneimine (PEI) is a positively charged water soluble block copolymer that directs cells morphology, and can be used to remove variations in cellular population/subpopulations [33]. ELP is a mammalian elastin made from amino acids including valine, proline, and glycine; it 
has previously been used in drug delivery and tissue engineering applications [34, 35]. Recent studies have shown that ELP-PEI successfully induces spheroid formation of rat hepatocytes [36]. The potential benefit of using ELP-PEI is to eliminate variations in hMSCs morphology as discussed in Chapter 4 [37]. Despite the shortcomings of currently available separation technologies, hMSCs therapeutics have demonstrated their healing potential.

\subsection{Successful hMSC Therapies and Insulin Producing MSCs}

The purpose of this section is to contextualize the power of using hMSCs for disease treatment. Medical researchers have focused on hMSCs impact on chronic diseases suffered by children. An 11-year-old and 2-year-old were both treated with hMSCs [30] for dilated cardiomyopathy, heart muscle disease in which the left ventricle becomes enlarged and cannot sufficiently pump blood to the body [38]. For the 11-year-old, $6 \mathrm{~mL}$ of autologous, meaning derived from the child's own tissue, therapeutic solution containing $4.8 \times 10^{6}$ cells $/ \mathrm{mL}$ of hMSCs were transplanted into the child using a catheter. hMSCs were transplanted into the proximal left main coronary artery and cardiac function improved from IV (initial) to II [30]. The 2-year-old's treatment differed slightly, a coronary balloon dilation catheter was placed in the left anterior artery, and low pressure was used for inflation. 270 million bone-marrow derived hMSCs were autologously transplanted into the child by an intracoronary bolus injection. The left ventricular injection fraction improved heart function from $24 \%$ to $45 \% 6$ months after the hMSC therapy 
[30]. These studies illustrate that hMSC therapies have been successful in children, and it is key to point out that a large number of cells were used for treatment, so extensive cell culture magnification and purification were conducted.

\section{(a) 6 Step Process}

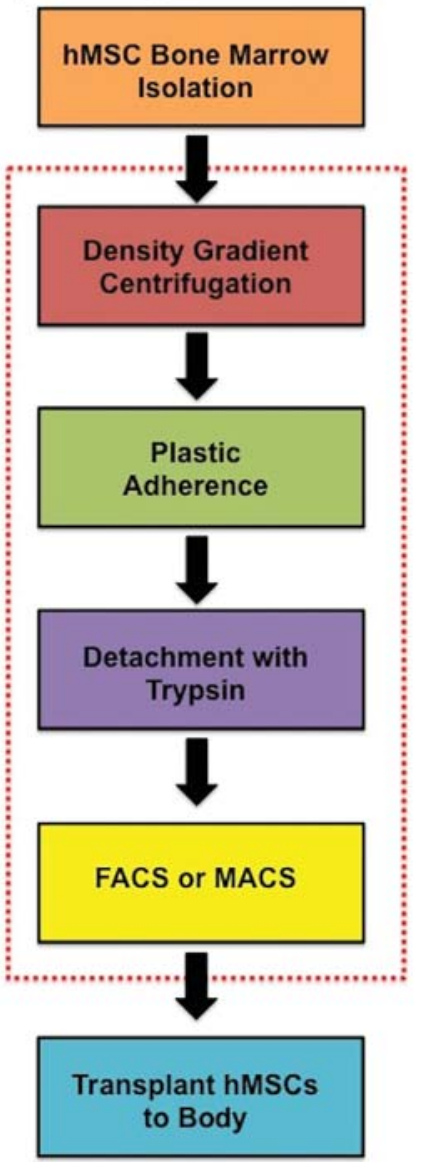

\section{(b) 3 Step Process}

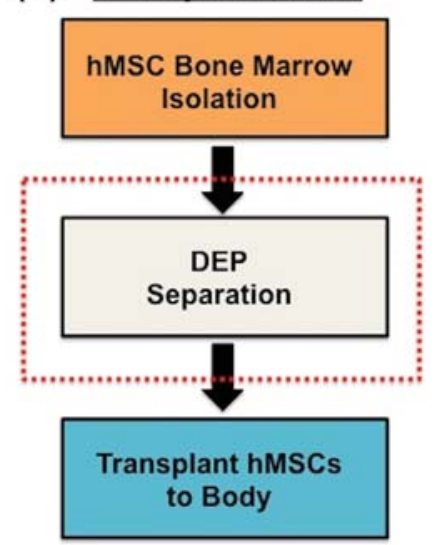

Figure 1.1 Flow diagrams outlining the steps necessary to achieve hMSC isolation using (a) density centrifugation with FACS or MACS and (b) the presently explored dielectrophoretic separation. 
Researchers from Tehran University in Iran have examined hMSCs differentiation into insulin producing cells, which would directly benefit children afflicted with type 1 diabetes. Their approach was to direct adipose-derived hMSCs toward insulin producing cells in a three-step cell culture protocol. In each step, the cell culture media was enhanced with glucose, alcohol, vitamin B3, and an insulin promoter peptide, exendin-4. The cells were treated with this enhanced media over the course of 24 days. Verification of insulin producing hMSCs was completed using dithizone staining, RT-PCR, immunochemistry, and morphology evaluation [39]. This work is important because it adds scientific evidence that hMSCs can potentially be used as a therapeutic option for diabetes treatment. A full review of hMSCs and their potential medical impact is in Chapter 2. Although hMSCs are promising in diabetes treatment, the current separation methods are labor and resource intensive, and have been shown to reduce viability such that extremely large concentrations of cells are required for effective therapies [40].

In summary, hMSCs have the potential to be used in clinical applications for disease treatment, but new methods for cell separation are needed to selectively isolate hMSCs from diverse cell populations. Therefore, the objective of this body of work is to develop a first generation technology for label-free, one-step, rapid hMSCs separation and purification without altering cellular function or reducing cell viability. This work outlines a new separation technique for improved hMSCs treatment efficacy that couples dielectrophoresis (DEP) and microfluidics to more efficiently separate hMSC populations. Additionally, this work developed a new 
DEP data collection approach by frequency sweeping which enables an automated data analysis method. These tools can work in tandem in a noninvasive manner to interrogate the exterior and interior cell structure and then cause translational motion harness-able for separations. These technologies also reduce the number of steps needed to achieve hMSC separation, as shown in Figure 1.1.

\subsection{Dielectrophoresis and Electrokinetics}

Dielectrophoresis (DEP) is a subset of a larger phenomenon called electrokinetics, which uses electrical energy as a driving force to induce particle motion [41]. DEP is polarization of neutral or charged particles or cells due to spatial non-uniformities in applied alternating current (AC) or direct current (DC) electric fields. For this work, DEP can function as a spatial separation technique that has potential to overcome the shortcomings of density gradient centrifugation, FACS, and MACS and could provide a rapid electrical signature for hMSCs. DEP technologies enable a variety of particle polarizations and manipulations with nonuniform AC electric fields on microchips $[42,43]$ to study cell systems such as red blood cells [44, 45], cancer cells [46, 47], white blood cells [48], and yeasts cells $[49,50]$. The advantages to coupling DEP with microfluidics are microliter sample

size, quick analysis ( $\sim$ minutes to achieve results), little sample preparation, and minimal waste production. Disadvantages are that extended electric field exposure times ( $>5$ mins) can negatively affect cell properties and viability [51]. 
DEP, also known as dielectric dispersion, is unique because in $\mathrm{AC}$ electric fields at specific frequencies, dipoles can be induced in or around particles and the permittivity of that particle changes with frequency [41, 52]. At low frequencies, this permittivity change is attributed to the polarization at the interface of the suspended particle, and for hMSCs this interface is equivalent to its membrane [42, 52]. At higher frequencies, the dielectric dispersion is influenced by the internal composition and structure of the particle; for hMSCs, cytoplasm properties play a critical role [42, $52,53]$. This frequency-dependent phenomenon allows for biological cell systems, such as hMSCs to be optimized for property-specific separations.

DEP utilizes nonuniform electric fields for cell movement based on the polarizability and dielectric properties (permittivity and conductivity) of their membrane, cytoplasm, and organelles [52]. Cells have distinct dielectric dispersions that can be used as an identification tool for cell purification, as depicted in Figure 1.2. A cell's complex permittivity is frequency dependent and characterized by the $\alpha$, $\beta$, and $\gamma$ dielectric dispersion regions $\left(\omega_{\alpha}<\omega_{\beta}<\omega_{\gamma}\right)$ [37]. At radio frequencies in the $\beta$-region, $0.10-10 \mathrm{MHz}$, the dielectric dispersion of cells is most heavily affected by their membrane; high frequencies penetrate a cell's surface and interrogates the internal structure [54]. Therefore, a variety of information can be obtained about a cell population, especially surface biomarkers, in the $\beta$-region. 


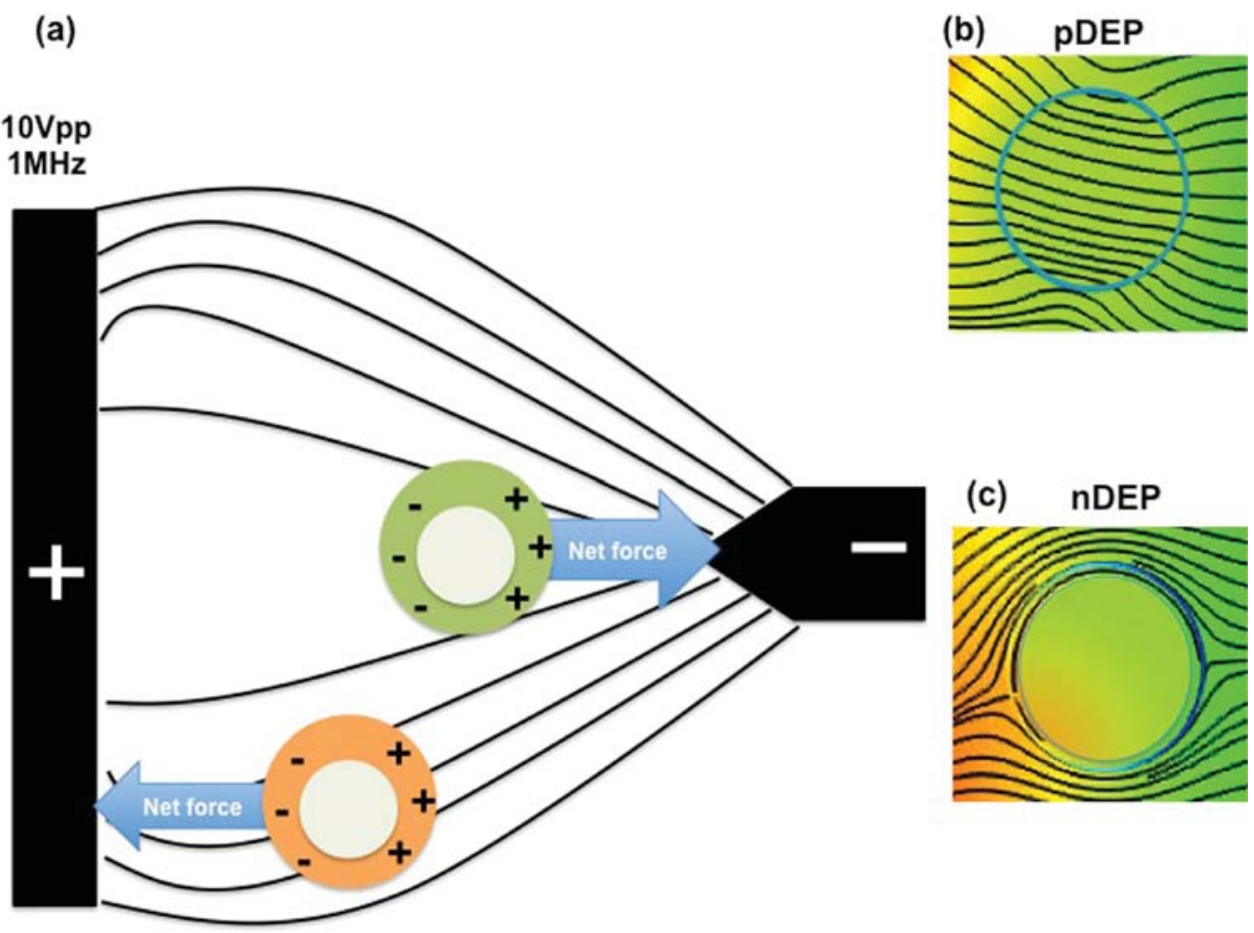

Figure 1.2 Nonuniform dielectrophoretic particle polarization. Cells with different membrane (crudely illustrated) and internal properties have different polarizations; as a consequence, spatial particle separations can be achieved.

In the $\beta$-region, Maxwell-Wagner interfacial polarizations dominate the overall cell polarization phenomena. Based on permittivity and conductivity, polarized cells will exhibit either positive DEP (pDEP) force Figure $1.2 b$, whereby cells move up the electric field gradient to high electric field gradient, or negative DEP (nDEP) force Figure 1.2c whereby cells are repelled from areas of high electric field density to move down the electric field gradient $[53,55]$. This cell motion in the spatially non-uniform electric field is defined by the Clausius-Mossotti factor, $\mathrm{f}_{\mathrm{CM}}$, for core-shell spherical particles $[52,53,56]$. 


$$
\begin{aligned}
& f_{C M}=\frac{\widetilde{\varepsilon}_{\text {cell }}-\widetilde{\varepsilon}_{\text {med }}}{\widetilde{\varepsilon}_{\text {cell }}+2 \widetilde{\varepsilon}_{\text {med }}}, \\
& \widetilde{\varepsilon}_{i}=\varepsilon_{i}+\frac{\sigma_{i}}{j \omega},
\end{aligned}
$$

where $\tilde{\boldsymbol{\varepsilon}}_{\boldsymbol{i}}$ is the complex permittivity of the cell $(\mathrm{i}=$ cell) and of the medium $(\mathrm{i}=$ med), which are dependent on the conductivity $(\sigma)$, permittivity $(\varepsilon)$, and angular frequency $(\boldsymbol{\omega})$ [53]. $f_{C M}$ is dependent on the complex permittivity of the cell and the medium, equation 2. If a cell experiences pDEP, then $f_{C M}$ (equation 1) is positive indicating the cell is more polarizable than the suspending medium, $\widetilde{\varepsilon}_{\text {cell }}>\widetilde{\varepsilon}_{\text {med }}$, and the cell moves toward areas of high electric field density [53]. For nDEP, the $f_{C M}$ is negative and the cell is less polarizable than the suspending medium, $\widetilde{\varepsilon}_{\text {med }}>\widetilde{\varepsilon}_{\text {cell }}$, and the cell moves towards areas of low electric field density [53]. When $f_{C M}$ is zero, known as the cross-over frequency [52], cells transition from experiencing nDEP to $\mathrm{pDEP}$ or pDEP to nDEP and demonstrate little or no motion in the electric field. This cross-over frequency is an important component of a cell's DEP spectra because initial estimates of cell dielectric properties can be estimated from this number. Equation 1 assumes a homogeneous, spherical particle, but more complex cell structures can be modeled for DEP polarizations by changing the shape and cell layers. A full review of DEP with modeling is in Chapter 3. 


\subsection{Microfluidic Technology}

Microfluidics is the manipulation of fluids and aqueous suspensions on the microscale [57]; devices can have many different components such as channels, chambers, reaction chambers, pumps, multiple layers, and electrodes. For this project, electrodes are utilized to induce cell motion as a means to simply and directly examine biological cell properties [57, 58]. Microfluidic systems are easily mounted on glass microscope slides or other optically transparent platforms using polydimethylsiloxane (PDMS) elastomeric castings of microscale features [59]. Microfluidics can be used for biosensors [60], cell separation [61], cell lysis [62], protein analysis [63], bacteria detection [64], and droplet formation [65]. Microfluidics can also be used to mimic organs for drug therapy optimization [66]. The overlap of these techniques with biological cell analysis enables new dimensions bioprocessing, characterizations, and separations. Each of these example processes can be performed on individual microdevices or combined together into one device to achieve multistep bioprocessing. Devices such as these are referred to as lab-on-achip and are increasingly more prevalent due to reduced costs and user-friendly operation. Lab-on-a-chip microdevices have already been developed to achieve cellbased assays like cell migration, proliferation and cell-to-cell signaling [58], and could be extended to quickly and effectively separate hMSCs.

The strategic combination of dielectrophoresis and microfluidic technology is a powerful tool to study cellular behavior because the microdevice can facilitate beneficial micro-environments for the cells. Other advantages to microdevices that 
incorporate dielectrophoretic phenomena are precise control on applied external or chemical forces, no mechanical parts necessary, small reagent consumption, rapid response time, reduced risk of sample contamination, higher reliability, reproducibility, low cost and the ability to run multistep processes in series or parallel on one device [16]. Many microdevices are being developed that incorporate both electrokinetic and microfluidic technologies that enable property measurements of specific biological cell systems and stem cells are an underexplored area in this field. Thus, this work explores the utility of electrokinetic and microfluidic technology as a solution to the challenges with current hMSC cell population purifications.

\subsection{Dielectrophoretic Microfluidic Devices and Diabetes Treatment}

To summarize, hMSCs are a potential therapeutic treatment for diabetes mellitus. The main challenge associated with hMSCs and other types of stem cells is the lack of a rapid, simple method to purify desired subpopulations from heterogeneous cell samples without altering cell function or reducing cell viability [40]. Also, hMSCs are a newly explored cell system so explorative studies are underway to understand their diverse functions within the body. Further, a database has not yet been developed to correlate cell surface biomarkers to differentiation behaviors or cell types. A microfluidic device utilizing DEP to discern differences in cell dielectric properties (and thus surface biomarker expression) has the potential to resolve the purification problems associated with hMSCs. 
Therefore the objectives of this body of work is to:

Objective 1: Characterize the dielectrophoretic response of hMSCs to determine the DEP spectra including the cross-over frequency.

Objective 1a: Standardize hMSCs morphology with ELP-PEI to reduce size-dependent DEP variations. Characterize the DEP spectra of ELP-PEI treated hMSCs, then compare to native hMSCs in order to identify conditions for cell separations. Subpopulation variations are expected to be based on molecular level expression.

Objective 2: From the experimentally derived DEP spectra obtained in Objective 1, model the dielectric properties of human mesenchymal stem cells using MATLAB and the Core-Shell Spherical DEP Polarization models. Use these models to calculate the dielectric properties of hMSCs.

Objective 2a: Correlate these properties with known phenotypical molecular expressions of hMSCs.

Objective 2b: Compare dielectric properties with other cell systems.

Objective 3: Develop a new frequency sweep rate data collection technique for rapid compilation of the frequency dependent DEP spectrum. Optimize sweep rate parameters with polystyrene beads and verify on red blood cells.

The two themes of this dissertation are to establish the dielectric signature of the novel hMSCs biological system and develop an alternative DEP experimental characterization and ultimately separation technique. The next chapters are literature 
reviews on hMSCs (Chapter 2) and dielectrophoresis (Chapter 3). The DEP spectra for hMSCs were determined and hMSCs $\varepsilon_{\text {membrane, }} \sigma_{\text {membrane, }}$ and $\mathrm{C}_{\text {membrane }}$ (dielectric properties) were quantified in Chapter 4. ELP-PEI was used to standardize hMSCs morphology and the effects of the polymer treatment were also explored in Chapter 4. This portion of the work demonstrates the first steps necessary toward the development of a continuous cell sorting microdevice. Chapter 5 demonstrates the development of a new rapid DEP data collection technique with semi-automated data analysis tested on polystyrene beads and red blood cells; a patent disclosure has been filed on this work, with the full patent application to be submitted in July 2014 . Finally, conclusions and a summary of the main findings of this work are summarized in Chapter 6. A side project not central to the theme of this dissertation was completed and published as a book chapter - this is included as Appendix A.

\subsection{References}

1. Center for Disease Control: Heart Disease Facts. [cited 2014 January]; Available from: http://www.cdc.gov/heartdisease/facts.htm.

2. Center for Disease Control: Stroke facts. [cited 2014 January]; Available from: http://www.cdc.gov/stroke/facts.htm.

3. Center for Disease Control: Diabetes research and statistics. [cited 2014 $\begin{array}{lll}\text { January]; } & \text { Available }\end{array}$ http://www.cdc.gov/diabetes/consumer/research.htm. 
4. American Diabetes Association: Fast facts data and statistics about diabetes. [cited 2014 January]; from: http://professional.diabetes.org/admin/UserFiles/0 - Sean/FastFacts March 2013.pdf.

5. O'Hagan, C., G. De Vito, and C.A.G. Boreham, Exercising prescription in the treatment of type 2 diabetes mellitus current practices, existing guidelines and future directions. Sports Medicine, 2013. 43: p. 39-49.

6. Diabetes. 2013 [cited 2014 March 23]; Available from: http://www.who.int/mediacentre/factsheets/fs312/en/.

7. Tonelli, M. and C. Wanner, Lipid management in chronic kidney disease: synopsis of the kidney disease: improving global outcomes 2013 clinical practice guidelines. Annals of Internal Medicine, 2014. 160: p. 182.

8. Lima, S.M.F., et al., Diabetes mellitus and inflammatory pulpal and periapical disease: a review. International Endodontic Journal, 2013. 46: p. 700-709.

9. Federation, I.D. IDF diabetes atlas. 2013 [cited 2014 March 25]; 6:[Available from: http://www.idf.org/diabetesatlas.

10. National Diabetes Statistics, 2011. 2013 [cited 2014 January 15]; Available from: http://diabetes.niddk.nih.gov/dm/pubs/statistics/ - fast.

11. Kolios, G. and Y. Moodley, Introduction to stem cells and regenerative medicine. Respiration, 2013. 85: p. 3-10. 
12. Caplan, A., Adult mesenchymal stem cells for tissue engineering versus regenerative medicine. Journal of Cellular Physiology, 2007. 213: p. 341347.

13. Caplan, A. and D. Correa, The MSC: An injury drugstore. Cell Stem Cell, 2011. 9: p. 11-15.

14. Stewart, M.C. and A.A. Stewart, Mesenchymal stem cells: characteristics, sources, and mechanisms of action. Veterinary Clinics of North America: Equine Practice, 2011. 27: p. 243-261.

15. Bobis, S., D. Jarocha, and M. Majka, Mesenchymal stem cells: characteristics and clinical applications. Folia Histochemica Et Cytobiologica, 2006. 44: p. 215-230.

16. Wu, H.W., C.C. Lin, and G.B. Lee, Stem cells in microfluidics. Biomicrofluidics, 2011. 5: p. 013401.

17. Salem, H.K. and C. Thiemermann, Mesenchymal stromal cells: current understanding and clinical status. Stem Cells, 2010. 28: p. 585-596.

18. Fuchs, E. and T. Chen, A matter of life and death: self-renewal in stem cells. European Molecular Biology Organization, 2013. 14: p. 39-48.

19. He, S., D. Nakada, and S.J. Morrison, Mechanisms of stem cell self-renewal. Annual Review of Cell and Development Biology, 2009. 25: p. 377-406.

20. Caplan, A. and J.E. Dennis, Mesenchymal stem cells as trophic mediators. Journal of Cellular Biochemistry, 2006. 98: p. 1076-1084. 
21. Wu, L., et al., Trophic effects of mesenchymal stem cells in chondrocyte cocultures are independent of culture conditions and cell sources. Tissue Engineering: Part A, 2012. 18: p. 1542-1551.

22. Pevsner, F., M., S. Levin, and D. Zipori, The origins of mesenchymal stromal cell heterogeneity Stem Cell Review and Reports, 2011. 7: p. 560-568.

23. Kolf, C.M., E. Cho, and R.S. Tuan, Biology of adult mesenchymal stem cells: regulation of niche, self-renewal and differentiation. Arthritis Research \& Therapy, 2007. 9: p. 204.

24. Williams, A.R. and J.M. Hare, Mesenchymal stem cells: biology,pathophysiology, translational findings, and therapeutic implications for cardiac disease. Circulation Research, 2011. 109: p. 923940.

25. Gonzalez-Gonzalez, M., et al., Current strategies and challenges for the purification of stem cells. Journal of Chemical Technology and Biotechnology, 2012. 87: p. 2-10.

26. Flanagan, L.A., et al., Unique dielectric properties distinguish stem cells and their differentiated progeny. Stem Cells, 2008. 26: p. 656-665.

27. Vykoukal, J., et al., Enrichment of putative stem cells from adipose tissue using dielectrophoretic field-flow fractionation. Lab on a Chip, 2008. 8: p. 1386-1393.

28. Parekkadan, B. and J.M. Milwid, Mesenchymal stem cells as therapeutics. Annual Review of Biomedical Engineering 2010. 12: p. 87-117. 
29. Mosna, F., L. Sensebe, and M. Krampera, Human bone marrow and adipose tissue mesenchymal stem cells: a user's guide. Stem Cells and Development, 2010. 19: p. 1449-1469.

30. Zheng, G.P., et al., Mesenchymal stem cells in the treatment of pediatric diseases. World Journal Pediatrics, 2013. 9: p. 197-211.

31. Haasters, F., et al., Morphological and immunocytochemical characteristics indicate the yield of early progenitors and represent a quality control for human mesenchymal stem cell culturing. Journal of Anatomy, 2009. 214: p. 759-767.

32. Darling, E.M., et al., Viscoelastic properties of human mesenchymallyderived stem cells and primary osteoblasts, chondrocytes, and adipocytes. Journal of Biomechanics, 2008. 41: p. 454-464.

33. Osborne, J.L., R. Farmer, and K.A. Woodhouse, Self-assembled elastin-like polypeptide particles. Acta Biomaterialia, 2008. 4: p. 49-57.

34. Janorkar, A.V., et al., The use of elastin-like polypeptide-polyelectrolyte complexes to control hepatocyte morphology and function in vitro. Biomaterials, 2008. 29: p. 625-632.

35. Turner, P.A., et al., A surface-tethered spheroid model for functional evaluation of 3T3-L1 adipocytes. Biotechnology and Bioengineering 2014. 111: p. 174-183.

36. Janorkar, A.V., et al., Use of three-dimensional spheroids of hepatocytederived reporter cells to study the effects of intracellular fat accumulation 
and subsequent cytokine exposure. Biotechnology and Bioengineering, 2011. 108: p. 1171-1180.

37. Adams, T.N.G., K.M. Leonard, and A.R. Minerick, Frequency sweep rate dependence on the dielectrophoretic response of polysytrene beads and red blood cells Biomicrofluidics, 2013. 7: p. 064114.

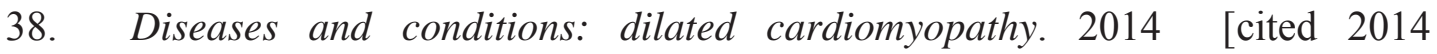
February 15]; Available from: http://www.mayoclinic.org/diseasesconditions/dilated-cardiomyopathy/basics/definition/con-20032887.

39. Moshtagh, P.R., S. Hojati Emami, and A.M. Sharifi, Differentiation of human adipose-derived mesenchymal stem cell into insulin-producing cells: an in vitro study. Journal of Physiology and Biochemistry 2013. 69: p. 451-458.

40. Posel, C., et al., Density gradient centrifugation compromises bone marrow mononuclear cell yield. PLOS One, 2012. 7: p. e50293.

41. Delgado, A.V., et al., Measure and intepretation of electrokinetic phenomena. Journal of Colloid and Interface Science, 2007. 309: p. 194-224.

42. Pethig, R., et al., Dielectrophoresis: A review of applications for stem cell research. Journal of Biomedicine and Biotechnology, 2010: p. 182581.

43. Zhang, C., et al., Dielectrophoresis for manipulation of micro/nano particles in microfluidic systems. Analytical and Bioanalytical Chemistry, 2010. 396: p. $401-420$. 
44. Leonard, K.M. and A.R. Minerick, Explorations of ABO-Rh antigen expressions on erythrocyte dielectrophoresis: Changes in cross-over frequency. Electrophoresis, 2011. 32(18): p. 2512-2522.

45. Srivastava, S.K., et al., Dielectrophoretic characterization of erythrocytes: Positive ABO blood types. Electrophoresis, 2008. 29(24): p. 5033-5046.

46. Salmanzadeh, A., et al., Dielectrophoretic differentiation of mouse ovarian surface epithelial cells, macrophages, and fibroblasts using contactless dielectrophoresis. Biomicrofluidics, 2012. 6(2): p. 13.

47. Salmanzadeh, A., et al., Isolation of prostate tumor initiating cells (TICs) through their dielectrophoretic signature. Lab on a Chip, 2012. 12(1): p. 182189.

48. Khoshmanesh, K., et al., Dielectrophoretic platforms for bio-microfluidic systems. Biosensors \& Bioelectronics, 2011. 26(5): p. 1800-1814.

49. Patel, S., et al., Microfluidic separation of live and dead yeast cells using reservoir-based dielectrophoresis. Biomicrofluidics, 2012. 6: p. 034102.

50. Razak, M.A.A., et al., Efficient dielectrophoretic cell enrichment using a dielectrophoresis-well based system. Biomicrofluidics, 2013. 7: p. 064110.

51. Lu, J., et al., Advancing practical usage of microtechnology: a study of the functional consequences of dielectrophoresis on neural stem cells. Integrative Biology, 2012. 4: p. 1223-1236.

52. Pethig, R., Review Article-Dielectrophoresis: Status of the theory, technology, and applications. Biomicrofluidics, 2010. 4(2): p. 35. 
53. Gagnon, Z.R., Cellular dielectrophoresis: Applications to the characterization, manipulation, separation and patterning of cells. Electrophoresis, 2011. 32(18): p. 2466-2487.

54. Martinsen, O.G., S. Grimnes, and H.P. Schwan, Interface phenomena and dielectric properties of biological tissue. Encyclopedia of Surface and Colloid Science, 2002.

55. Hawkins, B.G., et al., Automated dielectrophoresis characterization of mycobacterium smegmatis. Analytical Chemistry, 2011. 83: p. 3507-3515.

56. Demircan, Y., E. Ozgur, and H. Kulah, Dielectrophoresis: applications and future outlook in point of care. Electrophoresis, 2013. 34: p. 1008-1027.

57. Streets, A.M. and Y. HUang, Chip in a lab: microfluidics for next generation life science research. Biomicrofluidics, 2013. 7: p. 011302.

58. Kovarik, M.L., et al., Micro total analysis systems: fundamental advances and applications in the laboratory, clinic, and field. Analytical Chemistry, 2012. 85: p. 451-472.

59. McDonald, J.C. and G.M. Whitesides, Poly(dimethysiloxane) as a material for fabricating microfluidc devices. Accounts of Chemical Research, 2002. 35: p. 491-499.

60. Xiong, M., et al., Glucose microfluidic biosensor based on reversible enzyme immoblization on phottopatterend stimuli-responsive polymer. Biosensors \& Bioelectronics, 2013. 50: p. 229-234. 
61. Tan, S.J., et al., A microfluidic device to sort cells based on dynamic response to a stimulus. PLOS One, 2013. 8: p. e78261.

62. Watkins, N.N., et al., Microfluidic CD4(+) and CD8(+) T lymphocyte counters for point-of-care HIV diagnostics using whole blood. Science Translational Medicine, 2013. 5.

63. Peterson, V.M., et al., Ascites analysis by microfluidic chip allows tumor-cell profiling. Proceedings of the National Academy of Sciences of the United States of America, 2013. 110: p. E4978-E4986.

64. Jing, W.W., et al., Microfluidic device for efficient airborne bacteria capture and enrichment. Analytical Chemistry, 2013. 85: p. 5255-5262.

65. Lee, D.H. and J.K. Park, Reduction in microparticle adsorption using lateral interconnection method in a PDMS-based microfluidic device. Electrophoresis, 2013. 34: p. 3119-3125.

66. Sei, Y., et al., Engineering living systems on chips: from cells to humans on chips. Microfluidics Nanofluidics, 2014. 


\section{${ }^{1}$ Chapter 2 Human Mesenchymal Stem Cells and Type 1}

\section{Diabetes}

\subsection{Key Properties of Human Mesenchymal Stem Cells}

Stem cells are distinct unspecialized cells classified as embryonic or somatic (adult) and are of interest due to their ability to differentiate into specific cell types [1-3]. Embryonic stem cells are derived from embryos and somatic stem cells (mesenchymal, hematopoietic, neural, epithelial, and skin) are derived from other cells in the body excluding gametes. Stem cells are characterized by their differentiation potential with totipotent cells, being capable of differentiated into all cell types; pluripotent stem cells being capable of differentiating into almost all cells types; human embryonic stems cells most strongly demonstrate pluripotency. Somatic stem cells are multipotent meaning they only differentiate into closely related families of cells. Human mesenchymal stem cells (hMSCs) are somatic and ideal to work with because they are readily obtained from bone marrow and they have the ability to self-renew while undergoing differentiation [2]. hMSCs are an abundant source of cells found in various locations in the body including the umbilical cord [4-6], adipose tissue $[4,5,7,8]$, synovium $[5,7,9,10]$, periosteum $[5$, $11,12]$, and dental pulp $[5,13,14]$. hMSCs have the potential to differentiate into osteoblasts (bone cells), adipocytes (fat cells), chondrocytes (cartilage cells),

\footnotetext{
${ }^{1}$ The material contained in this chapter will be submitted for publication.
} 
astrocytes (neural cells), myoblasts (muscle cells), and Langerhans islets (pancreatic cells) based on environmental promoters $[2,13,14]$.

Presently there is not a database of unique biosurface markers that distinctly defines hMSCs or their progeny $[9,15,19-22]$, which is necessary for cell separation and purification to occur after sample isolation from the body. As a result, researchers have minimal understanding of the biological mechanisms that hMSCs display in therapeutics. Samples of hMSCs are isolated from bone marrow as heterogeneous mixtures [15]. In order for hMSCs to be highly efficient in cell therapy, isolated cells need to be purified. Therefore, new technology is needed that can accurately characterize hMSCs subpopulations and virility as well as reveal more information about their cellular function and biological mechanisms. Before proceeding to the technology for separating hMSCs, this chapter will review hMSCs key physical properties, elastin-like polypeptide polyethyleneimine (a morphology standardizing agent), current hMSC separation technologies, type 1 diabetes background, and set the foundation for dielectrophoresis as an alternative and superior separation technology.

\subsection{1 hMSC Self-Renewal, Differentiation Potential And Trophic} Activity

hMSCs are characterized by three key properties: (1) ability to self-renew, (2) trophic activity, and (3) differentiation potential, all of which are critical to their 
therapeutic potential. Figure 2.1 illustrates the three key properties of hMSCs and each is discussed separately in the following paragraphs.

Self-renewal is the process that hMSCs go through to create new stem cells [16]. Specifically, during self-renewal hMSCs divide to generate daughter cells with the same developmental potential as the mother cell. This property is not unique to stem cells, many cell types have similar self-renewal capabilities. There are two mechanisms for hMSC self-renewal division, symmetric and asymmetric [17]. During mitosis, the internal organelles of cells are equally parsed between two daughter cells to achieve symmetric division. The daughter cells have identical characteristics to the mother cell. In contrast, asymmetric division is the unequal parsing of cellular internal organelles between two daughter cells, resulting in cellular polarization [18]. The daughter cells display different characteristics from the mother cell. hMSCs continually undergo self-renewal [18]; differentiation into other cell types can concurrently occur.

Potency describes stem cell's ability to differentiate into other cell types. Cells are typically classified into one of three important levels of potency: totipotent, cells develop into any cell type; pluripotent, cells can form most cell types with the exception of placental tissues; and multipotent, cells can differentiate into many cells types, i.e. totipotent $>$ pluripotent $>$ multipotent [2]. The more potent a cell is, the more versatile and valuable it is for medical treatments. hMSCs are multipotent and differentiate into adipocytes, osteoblast, chondrocytes, and many other cells types [2]. For stem therapeutics, hMSCs can be selectively differentiated into specific cell 
types using growth factors. Controlling and subsequently purifying hMSC-derived cell types enables tailored autologous hMSCs disease treatment, are from and used to treat the same individual, disease treatments [3]. For example, osteogenic differentiation of hMSCs is accomplished by supplementing cell culture media with dexamethasone, ascorbic acid, and $\beta$-glycerolphosphate [19]. Adipocytes have been produced by treating hMSCs cell culture media with insulin, dexamethasone, isobutyl methyl xanthine, and rosiglitazone $[20,21]$. There is a different protocol utilized to direct hMSCs differentiation toward specific cell types. This type of cell manipulation can and has been implemented to make hMSCs an attractive cell source for diabetes mellitus treatment, as discussed in section 2.3.

The third key property of hMSCs is trophic activity, which is their ability to migrate to sites of injured tissues or to participate in response to injury by secreting growth factors [22]. Some examples of growth factors that hMSCs secrete are granulocyte colony-stimulating factor (G-CSF), a glycoprotein that stimulates bone marrow to produce granulocytes and stem cells and release them into the blood; leukemia inhibitory factor (LIF), a protein that influences cell growth; and transforming growth factor-beta 2 (TGF- $\beta 2$ ), a protein that performs many cellular functions [23-25]. Figure 2.1 illustrates the three key properties of hMSCs. 
(a)

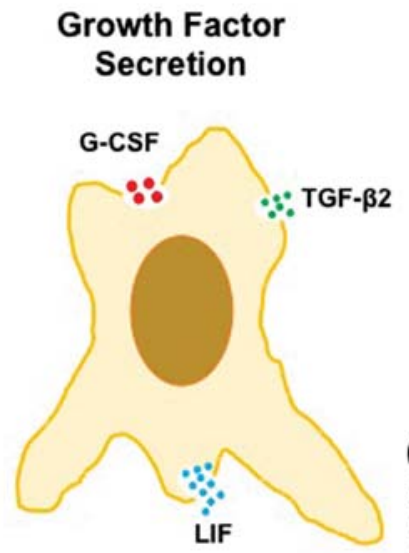

(b) Symmetric Division

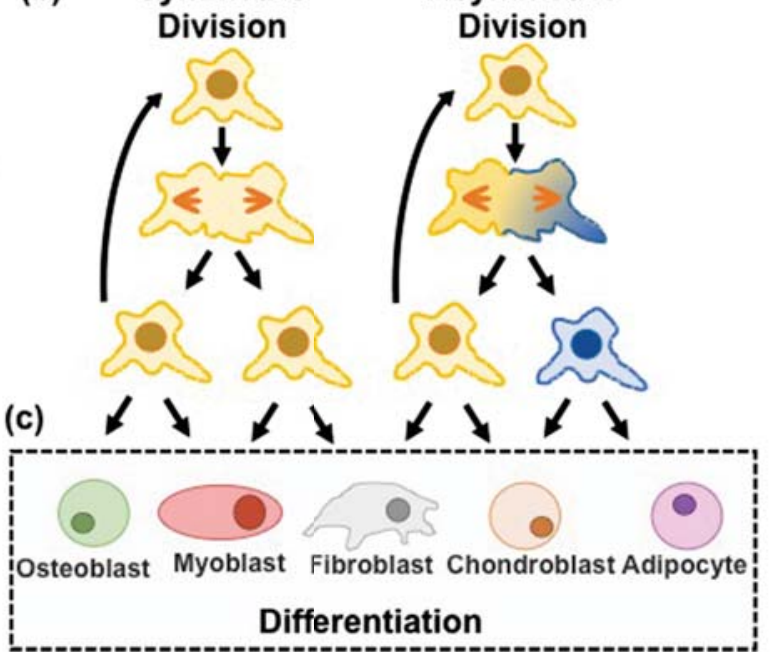

Figure 2.1 Important properties of hMSCs (a) trophic activity, (b) self-renewal, and (c) differentiation potential. Adapted from [2, 18, 23].

\subsection{2 hMSC Morphology}

hMSC morphology must be carefully considered when designing a microdevice to employ dielectrophoretic cell purification. According to Haasters et al., hMSCs can be assigned three morphological subpopulations: (a) rapidly selfrenewing cells, triangular or star-like shape; (b) elongated, fibroblastic-like, spindleshaped cells; and (c) slowly-replicating, large cuboidal or flattened cells [26]. Figure 2.2 shows microscope images of these subpopulations. The observed morphology is dependent on the cytoskeleton, cell adhesion, and active pathways; therefore the cell's morphology reveals information about cell fate and differentiation [26]. 


\subsubsection{Elastin-Like Polypeptide Polyethyleneimine}

To reduce variation in hMSCS morphology, elastin-like polypeptide (ELP) polyethyleneimine (PEI) was explored, and this cell shape variation reduction will assist with hMSCs separation. ELP-PEI is a postively charged block copolymer that directs cell morphology to a spheroidal shape. ELP is synthesized from amino acids valine, proline, and glycine, and has been previously used to produce $\mathrm{H} 35$ rat hepatocyte spheroids. ELP-PEI has also been used in studies to determine the affects of free fatty acids and cytokines in 2D and 3D rat hepatoma cell cultures [27, 28]. The affects of ELP-PEI on hMSCs are described in Chapter 4.
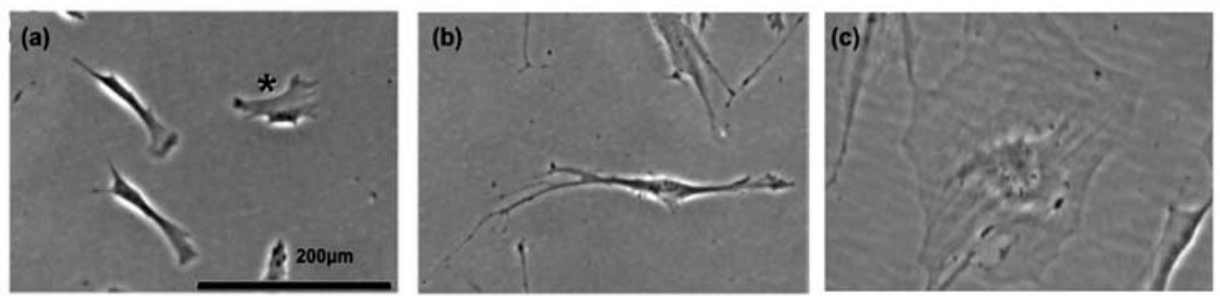

Figure 2.2 hMSCs morphological subpopulations (a) rapidly self-renewing, star like shape, (b) elongated fibroblastic-like spindle shaped cells, and (c) slowly-replicating flattened cells. Reprinted with permission from [26].

\subsection{4 hMSCs Surface Biosurface Markers}

Additionally, hMSCs can be characterized by a variety of surface proteins, which are major determiners of the cell phenotype, or the expressed individual cell characteristics. Scientists have extensively studied hMSCs phenotypes, but scientific 
publications do not provide a consistent catalog of surface proteins expressed on hMSCs surfaces. Currently, there is not a panel of unique surface proteins capable of characterizing hMSCs or distinguishing them from other cells. Flow cytometry has been used as a means to determine the phenotype of hMSCs using CD surface proteins. CD stands for cluster of differentiation and is the standard system by which newly discovered surface proteins are named [29].

Karaoz et al., conducted immunophenotyping on hMSCs using fluorescent tags for the following surface proteins CD3, CD4, CD5, CD7, CD8, CD10, CD11b, CD13, CD14, CD15, CD19, CD29, CD34, CD44, CD45, CD71, CD73, CD90, CD106, CD117, CD123, CD146, CD166, HLA-DR, HLA-ABC, and HLA-G. The results of this immunophenotyping yielded that hMSCs positively expressed CD10, CD13, CD29, CD44, CD73, CD90, CD106, CD146, CD166, and HLA-A,B,C. The hMSCs did not express the other 18 surface proteins examined (CD3, CD4, CD5, CD7, CD8, CD11b, CD14, CD15, CD19, CD34, CD45, CD71, CD90, CD106, CD117, CD123, HLA-DR, and HLA-G). Other researchers have done similar studies looking at the phenotype of hMSCs and Table 1 summarizes their findings. The proteins summarized in Table 1 are those that researchers had prior knowledge of and are not meant to be all-inclusive. The major conclusions from Table 1 are that hMSCs have a diverse phenotype with varying numbers and types of proteins expressed on their cell membrane surface. Figure 2.3 cartoons this phenotype diversity in hMSCs within one population; all cells do not exhibit the same surface proteins creating subpopulations. Further, a different technology is needed that can 
provide an alternative measure to distinguish hMSCs from other cells. Since there is not one unique surface protein, or group of proteins for hMSCs, it is advantageous to explore opportunities with dielectrophoresis that utilizes an electrical signature versus a protein signature.

Table 2.1 Summary of the hMSCs immunophenotyping [15, 30-33].

\begin{tabular}{llc}
\hline Function & Marker & hMSCs \\
\hline Cell Adhesion Molecule & $\begin{array}{l}\text { CD44, CD54, CD106, } \\
\text { CD146, CD90 }\end{array}$ & + \\
\hline $\begin{array}{l}\text { Bone Metabolism/ } \\
\text { Mineralization }\end{array}$ & $\begin{array}{l}\text { Osteonectin, } \\
\text { Osteopontin, } \\
\text { Osteoprotegerin, } \\
\text { Osteocalcin }\end{array}$ & - \\
Bone Mineralization & Biglycan & \\
Structural Protein of & $\begin{array}{l}\text { Procollagen-1, Collagen-I, } \\
\text { Cartilage }\end{array}$ & $\begin{array}{l}\text { Collagen-III, Collagen-V, } \\
\text { Collagen-VI }\end{array}$ \\
Structural Protein of & Collagen-II, Collagen-IX, & - \\
Cartilage & Collagen-X, Aggrecan, & \\
Glycoprotein & Decorin & \\
Integrin & CD9, CD59, CD161 & + \\
Leukocyte Antigen & CD29, CD73 & + \\
Anti-adhesive Molecule & HLA-A, HLA-B, HLA-C & + \\
Activated Leukocyte & Veriscan & - \\
CALLA & CD166 & + \\
TGF- $\beta$ & CD10 & + \\
RIKO-5 Recognition & CD105 & + \\
Aminopeptidase Recognition & CD55 & + \\
\hline
\end{tabular}




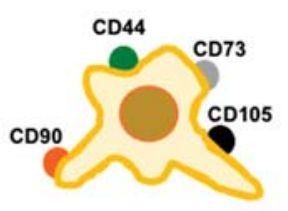

Subpopulation 1

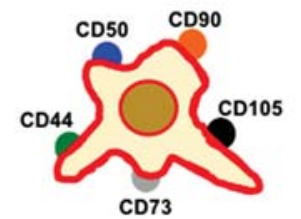

Subpopulation 2

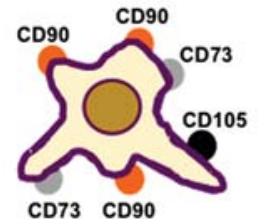

Subpopulation 3

Figure 2.3 Illustration of differentiation surface proteins present within one hMSCs population $[33,34]$.

At this point, the three main properties of hMSCs that make them ideal candidates for stem cell therapeutics, differentiation capacity, self-renewal, and trophic activity have been described. However, there is not one single or combination of unique physical hMSC properties that make them easily identifiable after bone marrow isolation. To achieve this goal, the International Society for Cellular Therapy put in place minimum requirements to label a cell population as hMSCs, which are [1,34]:

1) Plastic adherence,

2) Positive expression of CD105, CD73, and CD90,

3) Negative expression of CD34, CD45, CD11a, CD19, CD14, and HLA-DR,

4) Adipogenic, osteogenic, and chondrogenic differentiation potential.

The plastic adherence is a general property common in many cells and the specific surface proteins listed overlap with other cells, like hematopoietic stem cells. Further, it isn't possible to identify the propensity of individual cells toward the specialized cell type. Therefore, a more descriptive property would be beneficial for 
hMSC characterization and a separation tool to achieve quantification of the property would empower researchers to tailor cell populations for therapies.

The currently known mechanical and electrical properties of hMSCs are discussed in the following section. Knowledge of these key properties is critical for any hMSCs purification and identification efforts.

\subsection{5 hMSCs Mechanical Properties}

Mechanical properties typically measured on cells include elasticity, viscoelasticity, and strength (Young's modulus, tensile, compression, and stiffness). Thus far, hMSCs mechanical property studies have been dominated by elastic, viscoelastic, and Young's modulus measurements. These properties are exploited for separation and are important for therapeutic considerations. Elasticity is the measure of a cells tendency to return to its original form after deformation, similarly in viscoelasticity cells exhibit viscous and elastic characteristics under deformation [35]. In reviews [36-40], it's been concluded that the viscoelastic behavior of biological cells plays an important role in biophysical and biological responses to stimuli.

The goal of outlining the viscoelastic properties here is to demonstrate that hMSCs have unique physical properties that are useful for characterization and lends support to hMSCs having unique electrical markers. In the study completed by Darling et al. [35], the elasticity and viscoelasticity of two hMSC morphologies were examined: spherical and spread star-like. Spherical hMSCs deformation was 
measured at the cell's center, and the spread-like hMSCs deformation was measured over the cell's nucleus. The spread star-like hMSCs studied here are similar to those in Figure 2.2a, and the results are summarized in Table 2.2.

Table 2.2 Elasticity and Viscoelasticity of hMSCs with spherical and spread star-like morphologies [35].

\begin{tabular}{|c|c|c|c|c|}
\hline Morphology & Cell Type & $\mathrm{n}$ & Elasticity $(\mathrm{kPa})$ & Viscoelasticity (kPa) \\
\hline Spherical & hMSC & 53 & $2.5 \pm 1.8$ & $0.47 \pm 0.52$ \\
\hline Spread Star-like & hMSC & 67 & $3.2 \pm 2.2$ & $2.2 \pm 1.9$ \\
\hline
\end{tabular}

From Table 2.2, the elasticity for each hMSC morphology tested was a similar order of magnitude, $2.5 \mathrm{kPa}$ (spherical) versus $3.2 \mathrm{kPa}$ (spread star-like). However, the viscoelasticity changed by an order of magnitude, from $0.47 \mathrm{kPa}$ (spherical) to $2.2 \mathrm{kPa}$ (spread star-like). Titushkin et al. [41] corroborated hMSC spread star-like morphology with atomic force microscope indentation, and found the Young's Modulus to be $3.2 \mathrm{kPa}$.

Just as growth factors can be added to cell culture media to direct hMSCs differentiation, Kim at al.[42], found that hMSC populations are sensitive to mechanical vibrations. Stimulation at $30 \mathrm{~Hz}$ and $0.3 \mathrm{~g}$ of acceleration induces osteogenic differentiation. This mechanical vibration increased the alkaline phosphatase activity and calcium depositions, which are consistent with osteoblast differentiation. Similarly, Subramony et al. [43], coupled nanofiber alignment with 
tensile loading to induce fibroblastic differentiation. Controlling hMSCs differentiation using mechanical or other cues after purification is a powerful tool that can be utilized in hMSC therapeutics.

Lastly, supporting evidence by Alexopoulos et al. [44] and Guilak and Mow [45], illustrate that the mechanical properties of cells can affect their physical interactions with surrounding extracellular matrices (ECM). ECM is a complex arrangement of polysaccharides and proteins secreted by cells for structural support [29]. The interaction of hMSC derivatives with ECM is an important consideration as these cells move toward therapeutic applications.

These results show that mechanical properties may serve as a basis of characterization for cell subpopulations without necessitating cell tagging. The disadvantage to using elasticity measurements is that they may serve as a mechanical cue that triggers differentiation. Thus, mechanical characterizations can be classified as a more invasive tool. Further, it is necessary to perform individual cell mechanical property measurements in order to identify subpopulations prior to separation. The tools to conduct these individual cell measurements followed by separation do not currently exist. In slight contrast, electrical measurements can determine similar properties as mechanical, but are much more amenable to being conducted on individual cells. 


\subsection{6 hMSCs Electrical Properties}

The measurement of hMSC electrical properties, and their link to physical properties has included impedance measurements. In the last 20 years, minimal literature has been published on hMSCs electrical properties. For example, a search of 'human mesenchymal stem cells electrical properties' in Web of Science yields only 24 articles; only two articles directly measure hMSCs electrical characteristics. The remaining articles relevant to hMSCs can be categorized in the following manner: biocompatible materials (applications in scaffolds, nanofilms, nanotubes, polyimide electrodes) [46-50], electrical stimulation (to monitor membrane mechanics and calcium dynamics) [51-53], and hMSC differentiated progeny impedance measurements [54-56]. The most relevant publications to this dissertation involve impedance measurements of the hMSCs and are more reviewed in this section.

Impedance is a measure of the opposition to AC current flow in a circuit. When cells or cell suspensions are embedded into the circuit, information can be acquired about cell function and morphology. Hildebrandt et al., used impedance as an hMSC osteogenic differentiation indicator, and found that undifferentiated hMSCs and osteocytes have different impedance values, as reproduced in Figure 2.4a. The impedance of undifferentiated hMSCs is particularly relevant to this research; hMSC suspensions in cell culture media (roughly $1.7 \mathrm{~S} / \mathrm{m}$ ) yield impedance values of 7.5-7.9 $\times 10^{4} \Omega$ between $0.63-1.3 \mathrm{MHz}$ [55]. Cho et al., also used impedance

to monitor hMSC differentiation but was interested in adipocytes rather than 
osteocytes. Cho's work corroborates Hildebrandt's by also demonstrating an increase in impedance for hMSCs derived adipocytes as reproduced in Figure 2.4b; average hMSC impedance was $350 \Omega$ [54]. It's important to note that while these studies quantify an electrical property of hMSCs, that property is not translatable to cell separations. Impedance is a sensor tool, but does not induce any motion so is not amenable to use as a separation technique. These experimentally determined impedance values are correlated to hMSCs membrane capacitance in Chapter 4 as a comparison to our own hMSC experimental work.
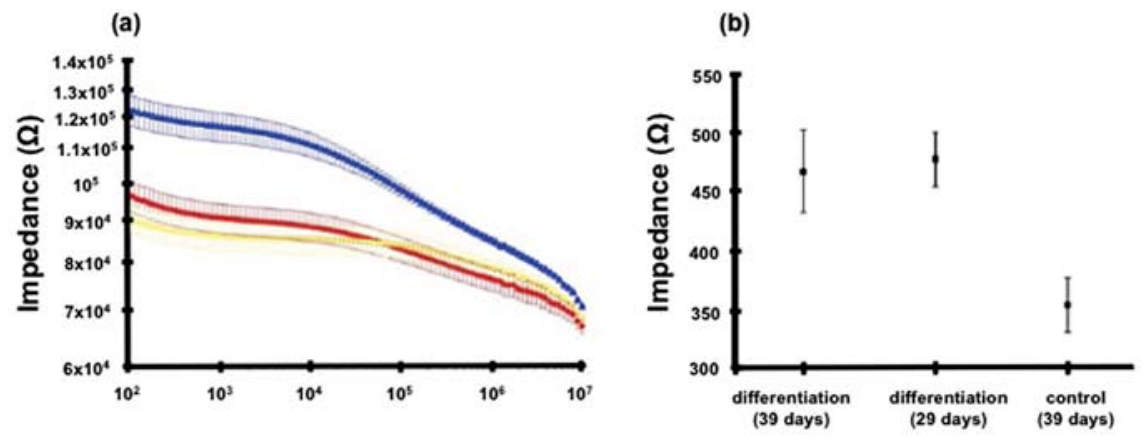

Figure 2.4 Impedance measurements for (a) [55] undifferentiated hMSCs (red) and osteocyte differentiated hMSCs (blue) (yellow is a nonrelated mikrozid liquid treatment to induce cell necrosis), and (b) [54] undifferentiated hMSCs (labeled control) and adipocyte differentiated hMSCs (labeled differentiation). Impedance increases in differentiated cells indicating differing morphology and physiological properties from the mother cells. Reprint permission requested from $[54,55]$. 
This review has thus far been restricted to hMSCs. However, other electrical measurements have been performed on other stem cell lines. Stem cell dielectrophoretic measurements are organized into Chapter 3, section 3.5 for continuity purposes.

In summary, there are no direct measurements of hMSCs membrane capacitance and permittivity, which are key properties for designing cell separations. This dissertation research seeks to fill this gap in knowledge by quantifying hMSCs membrane capacitance and permittivity using dielectrophoresis, a noninvasive electrical tool capable of cell translation and thus cell population sorting. Further, a polymeric membrane treatment, ELP-PEI is utilized to reduce hMSCs morphology variations in order to better isolate the contributions from mechanical and electrical properties and thus yield a more efficient hMSC purification technique for type 1 diabetes therapeutics.

\subsection{Human Mesenchymal Stem Cells Separation Technology}

\subsubsection{Research Motivation Statement}

hMSCs therapeutics are especially important for the continued advancement of medicine due to their differentiation capacity, self-renewal, and trophic activity. Highly purified hMSC samples are necessary for therapies to be effective and efficient. Thus, separation after bone marrow isolation is critical and has relied upon flow cytometry and centrifugation. The following subsections describe current technologies that are used to separate hMSCs from heterogeneous cell populations. 
Current technology disadvantages leave room for improvement and provide a motivation to explore other separation techniques.

Current hMSCs separation techniques can be generalized into two categories: affinity-based and physical-based. Affinity-based techniques utilize hMSCs biological properties, like membrane surface proteins (CD's), to selectively purify the cells. Technologies that implement this separation scheme are fluorescentactivated cell sorting (FACS) and magnetic-activated cell sorting (MACS). Physical-based techniques, like density gradient centrifugation, utilize hMSCs size and plastic adherence to achieve separations.

\subsubsection{Fluorescent and Magnetic Activated Cell Sorting for Cell}

\section{Separations}

Fluorescent-activated cell sorting (FACS) and magnetic-activated cell sorting (MACS) are separation techniques employed after hMSC extraction from the body and cell culture to then achieve purification. FACS uses unique cell-surface antigens as recognition elements to tag target cells. This cell labeling alters cellular function, which is not desirable for medical applications [57]. More specifically, FACS is a specialized type of flow cytometry that uses a laser beam to capture emergent light from passing cells. Based on the signal, fluidics are used to direct and sort cells into channels. The data collected reports information on cell size, complexity, phenotype, and health [58]. FACS has six main components: fluidic, laser, optic, detector, electronic and computer system. 
In a typical hMSC separation, cells are first labeled by incubating them with fluorescent conjugated antibodies (CD34, CD45, CD73, CD105 and CD166; conjugated with phycoerythrin or fluorescein isothiocyanate) [59] and then transported through the fluidic system for single cell laser analysis (for accurate data collection) via mechanical vibrations and hydrodynamic focusing [60]. The individual cells pass through the laser causing light to scattering in two ways: forward light scatter, where light is sent in the forward direction as it hits a cell; or side light scatter, where light is sent at a $90^{\circ}$ angle from the cell. Side scatter is caused by cell granularity and structural complexity [61]. Forward and side light scatter provide a one-dimensional and two-dimensional graphical representation of cell population, respectively [62]. Separate detectors translate the scattered light intensity into a voltage pulse, which is proportional to cell size. In the final step, hMSC FACS separations involve directing the cells into channels based on the scattered light signal. The fluidic stream containing the laser-interrogated cells flows past electrodes that selectively activate to induce cell electrophoretic motion so the cells can be selectively collected in separate tubes. FACS enables multiparametric analysis of hMSCs [63-65]. Figure 2.5 illustrates the FACS system.

There are some disadvantages associated with using FACS for separation including low throughput of 5000-70,000cells/s [58], high cost $(\$ 75,000+)$ [66], large electronic system (Becton Dickson FACSJazz, 125lbs, 21x20x20in) [67], skilled technician necessary for operation [60], sample contamination risk (due to sample preparation) [66], processing time (3-6hrs) [58], altered cell function/viability 
from antibody tagging [68]. Nevertheless, this method is one of the best available and has been used to successfully separate hMSCs by many researchers [69-73].

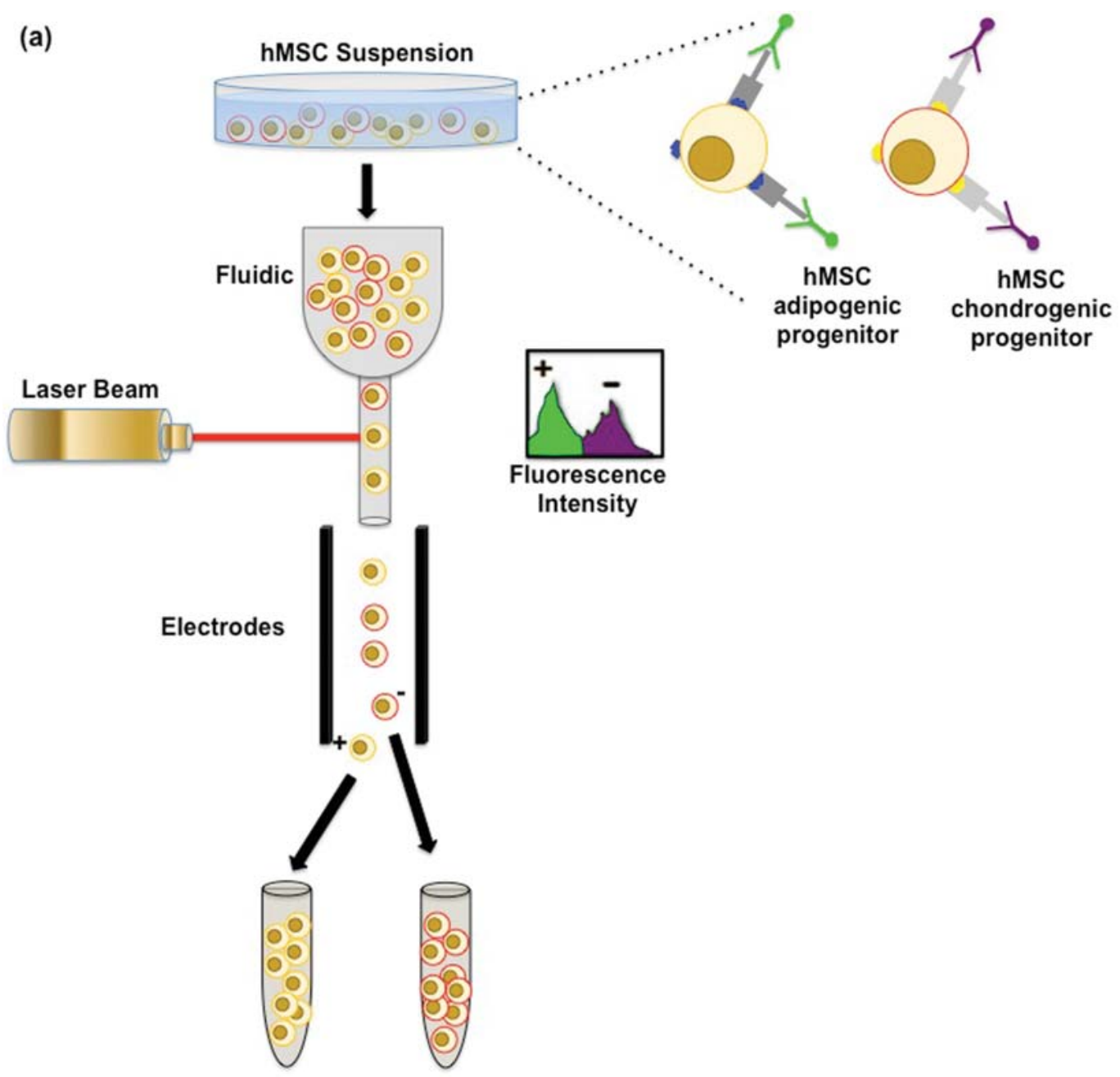

Figure 2.5 (a) Schematic of Fluorescent-Activated Cell Sorting and (b) illustrates labeling of two hMSCs progenitor cells. Adapted from [63-65]. Cells of interest are labeled with antigens and separated based on charge. 
MACS uses the same separation principles as FACS whereby target cells are labeled with antibodies (anti-immunoglobulin, anti-biotin, streptavidin, or antifluorochrome) conjugated with magnetic beads approximately $50 \mathrm{~nm}$ in size [74]. The cells are labeled through incubation that lasts approximately 30 mins [75]. After incubation, the cell suspension goes through a steel column placed in a magnetic field that impacts the magnetic beads bound to the cell surfaces. Cells are either positively or negatively selected based on the magnetic beads attached to the cell surface antibodies. Labeled cells stay in the column while the magnetic field is applied [76]. With the magnetic field on, the nonlabeled (unattached) cells pass through the column and are collected in a test tube; once the magnetic field is turned off, the labeled cells are eluted from the column and collected in a separate test tube $[74,75]$. Figure 2.6 shows a schematic of the MACS system.

The advantages to using MACS are high throughput [76], easy use [58], and lower cost than FACS [60]. The disadvantages associated with this separation technique are cell labeling alters cell function [57] and long process time [77]. Despite the drawbacks, MACS has been widely implemented as a separation method for hMSCs population control [78-82].

To summarize, FACS and MACS can achieve hMSC separation based on biosurface marker membrane expression; this approach is referred to as immunophenotyping [58]. FACS and MACS are widely used because there is not a better alternative. The disadvantages associated with them, namely cost, throughput and altered cell function, are widely acknowledged and underscore the need for 
improvements in cell separation. Microfluidic devices utilizing dielectrophoresis are technologies with the ability to discern cell types without any chemical labeling and without altering cell function [57]. These tools are explored within this work to improve hMSC, motivated in part by the success of other cell separations for clinical applications [83]. Before discussing dielectrophoresis in detail (Chapter 3), other separation techniques that do not incorporate cell antibody labeling are reviewed.

(a)

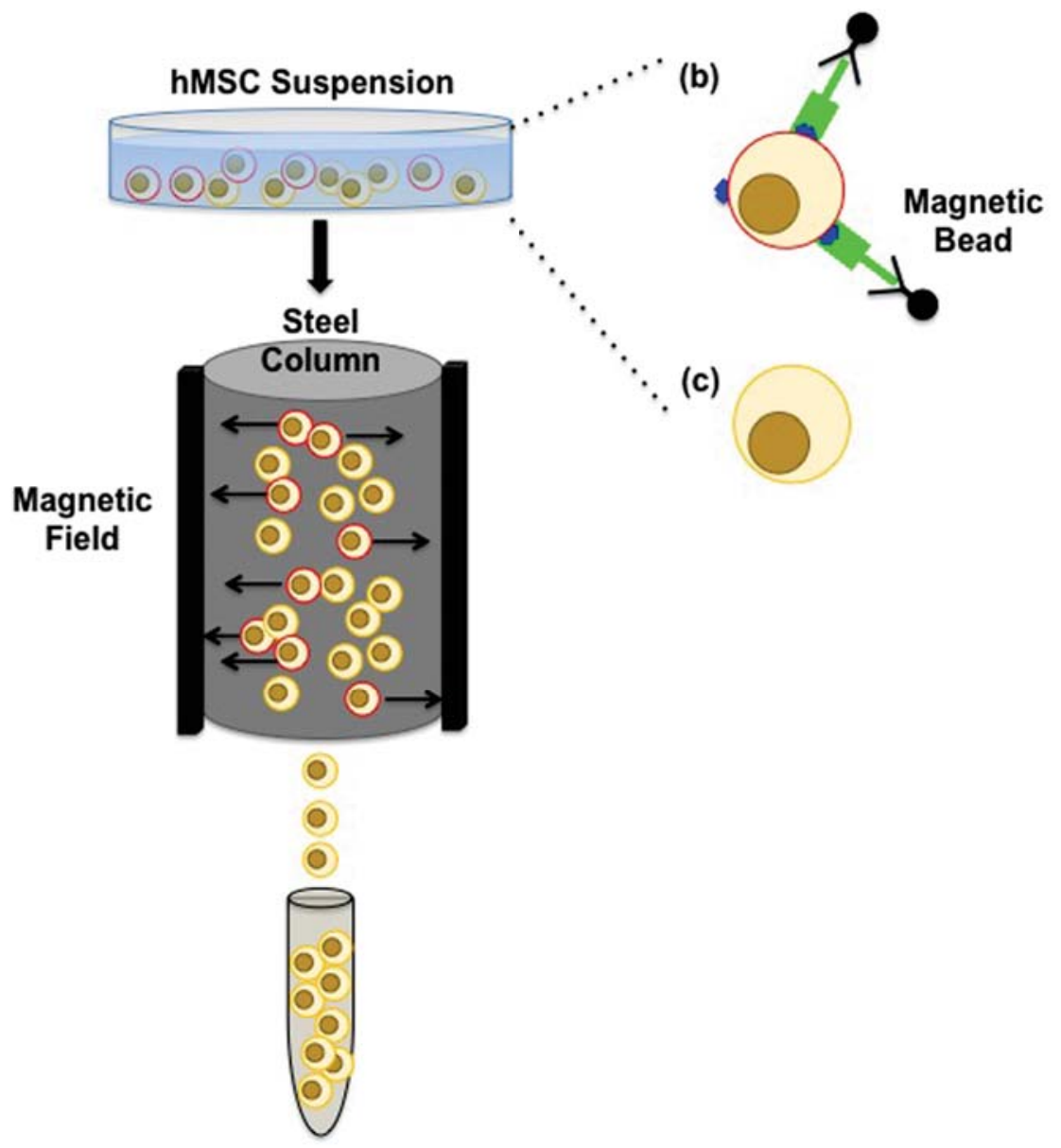

Figure 2.6 (a) Schematic of Magnetic-Activated Cell Sorting, and (b) cell labeled with magnetic beads, (c) unlabeled cell. Adapted from [74-76]. Target cells are 
labeled with antigen conjugated magnetic beads and with magnetic field on labeled cells are retained inside column, unlabeled cells pass through.

\subsubsection{Density Centrifugation and Membrane Filtration}

Density centrifugation is another separation technique employed to extract select hMSCs from a heterogeneous cell population. This method separates hMSCs from other cells based on their cellular density. A centrifuge tube is prepared by layering Percoll or Ficoll-Paque solutions from high density at the tube bottom to low density at the top, producing a gradient. After the gradient is poured, an hMSC suspension is placed in the centrifuge tube on top of the Percoll and centrifuged. During centrifugation, hMSCs migrate through the gradient until each cell reaches its isopycnic position [58], the position where the solution density equals the cell density. Increasing the number of density gradient layers increases separation efficiency [84].

Disadvantages with this method include: a) fluctuations in $\mathrm{pH}$ and osmolarity of density gradient solutions, which affect cell viability (changes may kill or reduce

cell efficacy) [58], b) density gradient preparation is laborious and tedious (layering in centrifuge tube) [85], and c) low selectivity (this is mainly employed as a preparative step before other separation methods are employed) [60].

Another physical based separation technique that has been used for hMSC separation is membrane filtration. This technique has been employed due to its fast processing time, easy use, and low cost. Separations are achieved based on cell size 
and affinity for adherence to a membrane surface $[8,86]$, as illustrated in Figure 2.7a. A membrane composed of rayon and polyethylene has been successfully used to filter hMSCs from mononuclear cells [87] as reproduced in Figure 2.7b.

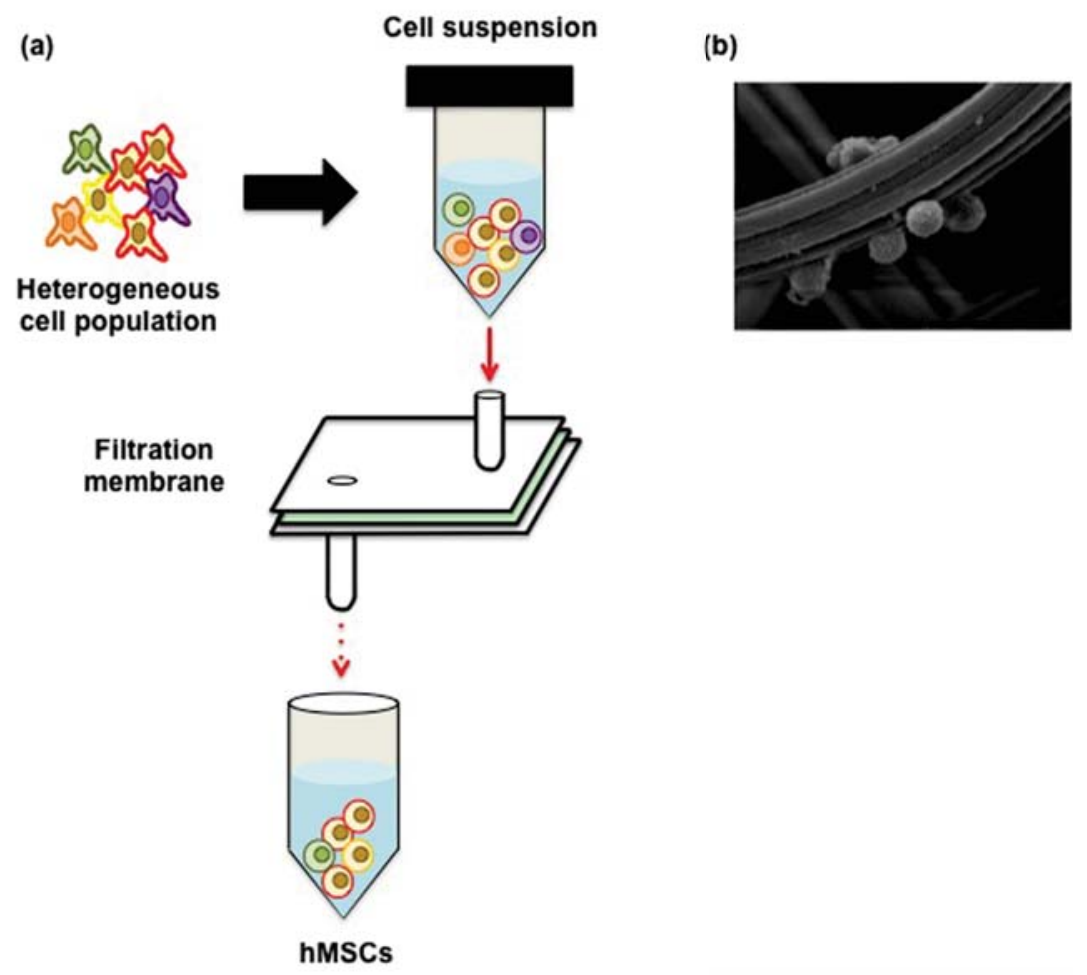

Figure 2.7 (a) Schematic of density gradient centrifugation [86] and (b) electron micrograph of nonwoven rayon and polyethylene with hMSCs attached. Permission requested for reuse [87].

The main objective of each of these technologies is to enrich hMSCs after bone marrow isolation. Each of the separation methods mentioned above have disadvantages that reduce efficiency in processing hMSCs for therapeutics, thus 
leaving room for improvement in hMSCs separation. Therefore, dielectrophoretic (DEP) microdevices are explored in this research as an alternative separation method. A review of DEP microdevice systems is given in Chapter 3 before presenting the experimental results of this dissertation (Chapter 5 and 6). In conclusion, the technology employed for hMSCs purification needs to be dynamic for scalability for higher throughputs and achieve high resolution for clinical therapeutic applications (one application is reviewed next), which require high concentrations of pure hMSCs.

\subsection{Type 1 Diabetes Mellitus}

\subsubsection{Definition}

Globally, there are 16,000 new cases of type 1 diabetes diagnosed each year [88]. Type 1 diabetes mellitus is a chronic autoimmune disease that diminishes the body's ability to produce the insulin hormone [89], as a resultant of beta cell destruction in the pancreas [90] as illustrated in Figure 2.8. Autoimmune diseases are

ones where the immune system perceives the body's own cells as foreign and destroys them [91]. The beta cells are specialized cells in the body that produce insulin in direct proportion to glucose in the blood stream [92]. The mechanism responsible for this autoimmune destruction of beta cells is currently unknown, but it leads to absolute insulin deficiency mediated by the immune system or it may be idiopathic [89]. This autoimmune destruction of beta cells in Type I is very different from Type II diabetes where food and body habits disrupt the balance and slowly 
wear out the functionality of the beta cells. hMSC therapies for autoimmune diseases are effective because they can replace destroyed cells and sometimes avoid being recognized as foreign by the immune system.

At the center stage of this disease is insulin production. The pancreas, a gland behind the stomach, secretes insulin into the blood stream and the insulin controls the amount of glucose in the blood via metabolic reaction pathways. Glucose is necessary for cells and tissues through the body to get the energy they need; an easily metabolized source of energy for the cells come from glucose consumed through food (bread, pasta, milk, rice, etc.). In type 1 diabetes, there is not enough insulin present to metabolize and transport the glucose from the blood into cells therefore, blood glucose concentrations increase causing complications [93].

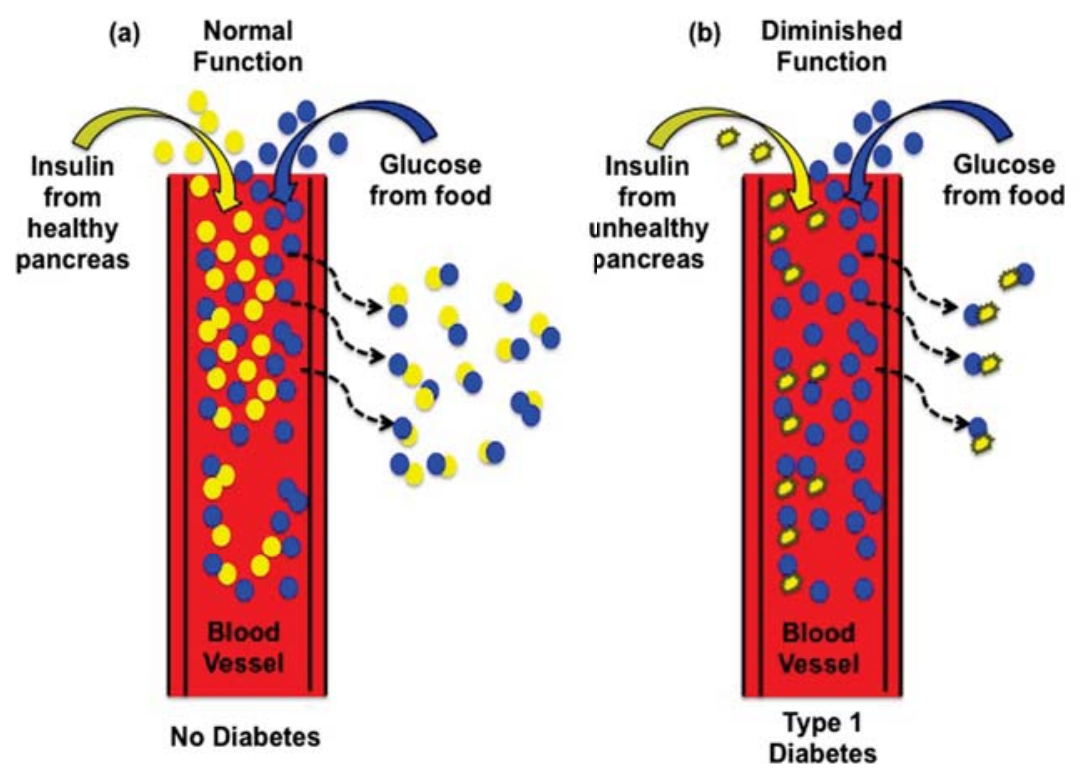

Figure 2.8 Schematic of Type 1 Diabetes (a) normal functioning pancreas and (b) diminished pancreas function, beta cells destruction. Beta cells within the pancreas 
do not produce the insulin necessary to reduce glucose concentrations in blood [89, 90].

Although the mechanism is unknown, researchers have tried to determine genetic predispositions to type 1 diabetes. $80 \%$ of children with IPEX (immune dysregulation, polyendocrinopathy, enteropathy, X-linked syndrome), characterized by a mutation in the Foxp3 transcription factor (protein involved in regulating the immune system [94]), develop type 1 diabetes [95-97]. Another gene mutation example is with autoimmune polyendoocrine syndrome type 1, qualified by a mutation in the AIRE transcription factor, resulting in $20 \%$ of children with this disease developing type 1 diabetes [98]. Other gene mutations that have shown susceptibility to type 1 diabetes are HLA (human leukocyte antigen) [99], INS (insulin) [100], PTPN22 (protein tyrosine phosphatase, non-receptor type 22) [101], IL2RA (interleukin-2 receptor alpha chain) [102], and CTLA-4 (cytotoxic Tlymphocyte antigen 4) [103]; all of these genes are associated with the function of the immune system.

Environmental factors have also been explored to understand more about type 1 diabetes. Data suggests that viral infections may precipitate type 1 diabetes, and correlations have been made between coxsackievirus (CVB4) [104], a virus that lives in the digestive tract and is a subset enterovirus. Increased amounts of neutralizing antibodies as a result of CVB4 were found in patients newly diagnosed with type 1 diabetes [105], and was confirmed with PCR (polymer chain reaction) 
genetic matching [106]. Yoon et al. [107], demonstrated with mouse subjects that CVB4 can infect beta cells causing insulitis and diabetes. In addition, Yoon also isolated CVB4 from a child with recent type 1 diabetes onset [108]. A contrasting study looked at the Pribilof Islands in Alaska after a CVB4 epidemic, and found that type 1 diabetes incidence was similar between infected and uninfected individuals [109], suggesting no connection between the diseases. Testing in the Finnish population provided positive links between individuals with enterovirus and type 1 diabetes $[110,111]$; this is corroboration in Figure 1.1 where incidence in Finland is greater than $24 \%$. Other viral infections linked to type 1 diabetes are rotavirus [112], cytomegalovirus [113, 114], parvovirus [115], and encephalomyocarditis [116], although all of these still need to be confirmed in larger patient populations [117].

Evidence has also shown that antibiotic and probiotic consumption may alter the gastrointestinal tract microbiota creating imbalance, which may lead to type 1 diabetes depending on intestinal composition [118]. It has also been found in the Sardinian population, that mycobacterium avium subspecies paratuberculosis, shed in infected cow's milk, was present in type 1 diabetes patients confirmed with blood tests [119]. In conclusion, there is growing evidence that links gene mutations, viruses, and bacteria to autoimmune disruption that causes type 1 diabetes. These factors and others require more exploration.

Although concrete factors that contribute to types 1 diabetes are still under investigation, diagnosis is well understood and completed via blood glucose assessments. A person with type 1 diabetes is characterized as having a casual blood 
glucose level $>200 \mathrm{mg} / \mathrm{dL}$, fasting blood glucose level $\geq 126 \mathrm{mg} / \mathrm{dL}$, or blood glucose level $>200 \mathrm{mg} / \mathrm{dL} 2$ hours after eating [89]. Currently, type 1 diabetes management requires insulin pens, shots, or pumps $[120,121]$. Type 1 diabetes complications include cardiovascular disease [122], neuropathy (nerve damage) [123], nephropathy (kidney damage) [124], retinopathy (eye damage) [125, 126], osteoporosis (bone degradation) [127], hearing impairment [128], and high-risk pregnancies [129].

\subsection{2 hMSCs as a Type 1 Diabetes Treatment Option}

Currently, there is no cure for type 1 diabetes, and the various complications necessitate the need for a curative therapeutic option, which is much more than simply an insulin management strategy. An effective cure will preserve remaining beta cells [130], and protect newly produced beta cells from autoimmune destruction in the pancreas [34]. Demonstration of this beta cell replacement therapy concept has been achieved through human pancreas transplantation in type 1 diabetes patients, which reestablished their insulin-independency [130]. However, this method cannot be widely implemented for all type 1 diabetes patients due to the number organs necessary to cure all patients. Further, organ transplant approaches are extremely invasive, autoimmune rejection of transplanted organs must be carefully managed to avoid rejection or death and combined leads to exorbitant medical bills. Thus, researchers have explored hMSCs as a much less invasive therapeutic option for beta cell replacement therapy. 
hMSCs were transplanted into diabetic immunodeficient mice following which the mice's beta cell numbers and insulin levels increased [131, 132]. Another animal model conducted by Ho et al., induced mice with diabetes using streptozocin to kill the beta cells and then subsequently treated them with multiple high MSC (non-human) concentrated injections. Multiple MSC injections were used because blood glucose homeostasis was not established after a single injection. Multiple injections gradually stabilized blood glucose to normal levels [133].

Others have employed chemical based methods and found that hMSCs differentiate into insulin producing cells [134-136], and those cells are able to release insulin in a glucose-dependent manner [137]. MSC differentiation toward insulin producing cells is mediated with additives in the cell culture procedure. Hisanga et al. [138], used activin A and betacellulin to direct murine (rat) MSCs differentiation; insulin producing cells were detected within 14 days of additive addition. Genetic modification with pancreatic duodenal homeobox-1 has also been used to differentiate hMSCs toward insulin producing cells $[139,140]$.

In summary, there are multiple methods, chemical and genetic modification, in place to differentiate hMSCs toward insulin producing cells for type 1 diabetes therapeutics. Additionally, hMSCs without directed differentiation toward insulin producing cells have also been used to improve diabetic condition, thus implicating hMSCs differentiate into insulin producing cells without external stimulus inside the body. These methods all indicate that a type 1 diabetes cure with hMSCs is possible, and present evidence that efficient cell purification is necessary for such therapeutic 
interventions. This provides exciting motivation to implement nondamaging dielectrophoretic microdevice-based separations for hMSC purification.

\subsection{Conclusions}

hMSCs hold high promise as a therapeutic treatment option for type 1 diabetes due to their differentiation ability, replenishing capacity, and their trophic activity. They have been shown to differentiate into insulin producing cells through a variety of methods: chemical, genetic, and undifferentiated. hMSCs are easily obtained from bone marrow as a heterogeneous population and therefore require separation before utilization in type 1 diabetes therapeutics. Within a single hMSC population, there are a variety of progenitor cells present (adipogenic-progenitor, osteogenic-progenitor, chondrogenic-progenitor, etc.) and these cells have a propensity to differentiate toward a specific cell type. Additionally, hMSCs are not well characterized because there is not a unique set of biosurface markers available that distinguishes hMSCs from other cell populations. To compensate, current methodologies utilize density centrifugation paired with either FACS or MACS to achieve hMSC subpopulation separations. These methods are disadvantageous because of operation time, high cost, and cell antigen labeling which has been shown to alter cell function. These multistep separation processes are not ideal for the scaleup required by clinical applications; multiple hMSCs transplantations of millions of cells are needed for effective therapeutic treatments. Therefore, this research seeks to 
solve the following issues associated with hMSCs separations and ultimately advance type 1 diabetes hMSC therapeutics:

1) No unique set of biosurface markers currently exists to distinguish hMSCs from other cell populations; mappings of CD proteins overlap with other cell systems. Instead, minimum requirements have been established by the International Society for Cellular Therapy.

2) Morphology variations within hMSCs populations make highly selective separations difficult.

3) FACS and MACS are expensive, low throughput, and timeconsuming separation techniques that alter cellular function.

\subsubsection{Dielectrophoresis for hMSC Separation}

Dielectrophoresis (DEP) is the separation technology explored in this research to resolve issues 1-3 associated with hMSC cell purification. DEP may be able to recognize a dielectric marker via cell membrane capacitance measurements making it possible to discern hMSCs from other cell populations. DEP is a quick, noninvasive separation method, with the potential to be low cost, and does not alter or damage cells [141]. Chapter 3 provides a review of DEP and the cell separations this technology has been able to accomplish. 


\subsection{References}

1. Salem, H.K. and C. Thiemermann, Mesenchymal stromal cells: current understanding and clinical status. Stem Cells, 2010. 28: p. 585-596.

2. Wu, H.W., C.C. Lin, and G.B. Lee, Stem cells in microfluidics. Biomicrofluidics, 2011. 5: p. 013401.

3. Spencer, N.D., J.M. Gimble, and M.J. Lopez, Mesenchymal stromal cells: past, present, and future. Veterinary Surgery, 2011. 40: p. 129-139.

4. Sarugaser, R., et al., Human mesenchymal stem cells self-renew and differentiate according to a deterministic hierarchy. PLOS One, 2009. 4: p. e6498.

5. Kim, Y.H., et al., Characterization of different subpoulations from bonemarrow-derived mesenchymal stromal cells by alkaline phosphatase expression. Stem Cells and Development, 2012. 21: p. 2958-2968.

6. Jang, Y.K., et al., Optimization of the therapeutic efficacy of human umbilical cord blood-mesenchymal stromal cells in an NSG mouse xenograft model of graft-versus-host disease. Cytotherapy, 2014. 16: p. 298-308.

7. Pelttari, K., E. Steck, and W. Richter, The use of mesenchymal stem cells for chondrogenesis. Injury-International Journal of the Care of the Injured, 2008. 39: p. S58-S65.

8. $\quad \mathrm{Wu}, \mathrm{C} . \mathrm{H} .$, et al., The isolation and differentiation of human adipose-derived stem cells using membrane filtration. Biomaterials, 2012. 33: p. 8228-8239. 
9. Pei, M., F. He, and G. Vunjak-Novakovic, Synovium-derived stem cell-based chondrogenesis. Differentiation, 2008. 76: p. 1044-1056.

10. Morito, T., et al., Synovial fluid-derived mesenchymal stem cells increase after intra-articular ligament injury in humans. Rheumatology, 2008. 47: p. $1137-1143$.

11. Wang, X., et al., Role of mesenchymal stem cells in bone regeneration and fracture repair: a review. International Orthopaedics, 2013. 37: p. 24912498.

12. Evans, S.F., et al., Solid-supported lipid bilayer to drive stem cell fate and tissue architecture using periosteum derived progenitor cells. Biomaterials, 2013. 34: p. 1878-1887.

13. Tirino, V., et al., Methods for the identification, charaterization and banking of human DPSCs: current strategies and perspectives. Stem Cell Review and Reports, 2011. 7: p. 608-615.

14. Ji, E.H., et al., Viability of pulp stromal cells in cryopreserved deciduous teeth. Cell and Tissue Banking, 2014. 15: p. 67-74.

15. Kolf, C.M., E. Cho, and R.S. Tuan, Biology of adult mesenchymal stem cells: regulation of niche, self-renewal and differentiation. Arthritis Research \& Therapy, 2007. 9: p. 204.

16. Lodi, D., T. Iannitti, and B. Palmieri, Stem cells in clinical practice: applications and warnings. Journal of Experimental and Clinical Cancer Research, 2011. 30. 
17. He, S., D. Nakada, and S.J. Morrison, Mechanisms of stem cell self-renewal. Annual Review of Cell and Development Biology, 2009. 25: p. 377-406.

18. Fuchs, E. and T. Chen, A matter of life and death: self-renewal in stem cells. European Molecular Biology Organization, 2013. 14: p. 39-48.

19. Halvorsen, Y.D.C., et al., Extracellular matrix mineralization and osteoblast gene expression by human adipose tissue-derived stromal cells. Tissue Engineering, 2001. 7: p. 729-741.

20. Pittenger, M.F., et al., Multilineage potential of adult human mesenchymal stem cells Science, 1999. 284: p. 143-147.

21. Vallee, M., J.F. Cote, and J. Fradette, Adipose-tissue engineering: taking advantage of the properties of human adipose-derived stem/stromal cells. Pathologie Biologie, 2009. 57: p. 309-317.

22. Caplan, A. and J.E. Dennis, Mesenchymal stem cells as trophic mediators. Journal of Cellular Biochemistry, 2006. 98: p. 1076-1084.

23. Caplan, A., Adult mesenchymal stem cells for tissue engineering versus regenerative medicine. Journal of Cellular Physiology, 2007. 213: p. 341347.

24. Aghajanova, L., Leukemia inhibitory factro and human embryo implantation Annals of the New York Academy of Sciences, 2004. 1034: p. 176-183.

25. Parham, P., The Immune System. 3rd ed. 2009, New York: Garland Science.

26. Haasters, F., et al., Morphological and immunocytochemical characteristics indicate the yield of early progenitors and represent a quality control for 
human mesenchymal stem cell culturing. Journal of Anatomy, 2009. 214: p. 759-767.

27. Turner, P.A., et al., A surface-tethered spheroid model for functional evaluation of 3T3-L1 adipocytes. Biotechnology and Bioengineering 2014. 111: p. 174-183.

28. Janorkar, A.V., et al., Use of three-dimensional spheroids of hepatocytederived reporter cells to study the effects of intracellular fat accumulation and subsequent cytokine exposure. Biotechnology and Bioengineering, 2011. 108: p. 1171-1180.

29. Alberts, B., et al., Molecular Biology of The Cell. 5 ed. 2008, New York: Garland Science.

30. Hilfiker, A., et al., Mesenchymal stem cells and progenitor cells in connective tissue engineering and regenerative medicine: is there a future for transplantation? Langenbeck's Archives of Surgery, 2011. 396: p. 489-497.

31. Izadpanah, R., et al., Biologic properties of mesenchymal stem cells derived from bone marrow and adipose tissue. Journal of Cellular Biochemistry, 2006. 99: p. 1285-1297.

32. Karaoz, E., et al., A comprehensive characteristization study of human bone marrow MSCs with an emphasis on molecular and ultrastructural properties Journal of Cellular Physiology, 2010. 226: p. 1367-1382. 
33. Pountos, I., et al., Mesenchymal stem cell tissue engineering: techniques for isolation, expansion and application. Injury-International Journal of the Care of the Injured, 2007. 38S4: p. S23-S33.

34. Zheng, G.P., et al., Mesenchymal stem cells in the treatment of pediatric diseases. World Journal Pediatrics, 2013. 9: p. 197-211.

35. Darling, E.M., et al., Viscoelastic properties of human mesenchymallyderived stem cells and primary osteoblasts, chondrocytes, and adipocytes. Journal of Biomechanics, 2008. 41: p. 454-464.

36. Costa, K.D., Single-cell elastography: probing for disease with the atomic force microscope. Disease Markers, 2003. 19: p. 139-154.

37. Guilak, F., The deformation behavior and viscoelastic properties of chondrocytes in articular cartilage. Biorheology, 2000. 37: p. 27-44.

38. Huang, H.D., R.D. Kamm, and R.T. Lee, Cell mechanics and mechanotransduction: pathways, probes, and physiology. American Journal of Physiology-Cell Physiology, 2004. 287: p. C1-C11.

39. Ingber, D.E., Mechanobiology and diseases of mechanotransduction. Annals of Medicine, 2003. 35: p. 564-577.

40. Zhu, C., G. Bao, and N. Wang, Cell mechanics: mechanical response, cell adhesion, and molecular deformation. Annual Review of Biomedical Engineering, 2000. 2: p. 189-226.

41. Mathieu, P.S. and E.G. Loboa, Cytoskeletal and focal adhesion influences on mesenchymal stem cell shape, mechanical properties, and differentiation 
down osteogenic, adipogenic, and chondrogenic pathways. Tissue Engineering: Part B, 2012. 18: p. 436-444.

42. Kim, I.S., et al., Human mesenchymal stromal cells are mechanosenstive to vibration stimuli. Journal of Dental Research, 2012. 91: p. 1135-1140.

43. Subramony, S.D., et al., The guidance of stem cell differentiation by substrate alignment and mechanical stimulation. Biomaterials, 2013. 34: p. 1942-1953.

44. Alexopoulos, L.G., L.A. Setton, and F. Guilak, The biomechanical role of the chondrocyte pericellular matrix in articular cartilage Acta Biomaterialia, 2005. 1: p. 317-325.

45. Guilak, F. and V.C. Mow, The mechanical environment of the chondrocyte: a biphasic finite element model of cell-matrix interactions in articular cartilage. Journal of Biomechanics, 2000. 33: p. 1663-1673.

46. Damaraju, S.M., et al., Structural changes in PVDF fibers due to electrospinning and its effecrts on biological function Biomedical materials, 2013. 8(4): p. 045007.

47. Shahini, A., et al., 3D conductive nanocomposite scaffold for bone tissue engineering. International Journal of Nanomedicine, 2014. 9: p. 167-181.

48. Ku, S., M. Lee, and C.B. Park, Carbon-based nanomaterials for tissue engineering. Advance Healthcare Materials, 2013. 2(2): p. 244-260.

49. Singh, N., et al., Ionic liquids-based processing electrically conducting chitin nanocomposite scaffolds for stem cell growth. Green Chemistry, 2013. 15(5): p. 1192-1202. 
50. Prabhakaran, M.P., L. Ghasemi-Mobarakeh, and S. Ramakrishna, Electrospun composite nanofibers for tissue regeneration. Journal of Nanoscience and Nanotechnology, 2011. 11(4): p. 3039-3057.

51. Balint, R., N.J. Cassidy, and S.H. Cartmell, Electrical stimulation: a novel tool for tissue engineering. Tissue Engineering Part B-Reviews, 2013. 19(1): p. $48-57$.

52. Titushkin, I. and M. Cho, Regulation of cell cytoskeleton and membrane mechanics by electric field: role of linker proteins. Biophysical Journal, 2009. 96(2): p. 717-728.

53. Sun, S., et al., Physical manipulation of calcium oscillations facilitates osteodifferentiation of human mesenchymal stem cells. FASEB Journal, 2007. 21(7): p. 1472-1480.

54. Cho, S., E. Gorjup, and H. Thielecke, Chip-based time-continuous monitoring of toxic effects on stem cell differentiation. Annals of AnatomyAnatomischer Anzeiger, 2009. 191(1): p. 145-152.

55. Hildebrandt, C., et al., Detection of the osteogenic differentiation of mesenchymal stem cells in $2 D$ and $3 D$ cultures by electrochemical impedance spectroscopy. Journal of Biotechnology, 2010. 148(1): p. 83-90.

56. Wenisch, S., et al., Immunochemical, ultrastructural and electrophysiological investigations of bone-derived stem cells in the course of neuronal differentiation. Bone, 2006. 38(6): p. 911-921. 
57. Flanagan, L.A., et al., Unique dielectric properties distinguish stem cells and their differentiated progeny. Stem Cells, 2008. 26: p. 656-665.

58. Gonzalez-Gonzalez, M., et al., Current strategies and challenges for the purification of stem cells. Journal of Chemical Technology and Biotechnology, 2012. 87: p. 2-10.

59. De Becker, A., et al., Migration of culture-expanded human mesenchymal stem cells through bone marrow endothellum is regulated by matric metalloproteinase-2 and tissue inhibitor of metalloproteinase-3. Haematologica-The Hematology Journal, 2007. 92(4): p. 440-449.

60. Diogo, M.M., C. Lobato de Silva, and J.M.S. Cabral, Separation technologies for stem cell bioprocessing. Biotechnology and Bioengineering, 2012. 109: p. 2699-2709.

61. Wilkerson, M.J., Principles and applications of flow cytometry and cell sorting in companion animal medicine. Veterinary Clinics of North America - Small Animal Practice, 2012. 42(1): p. 53.

62. Herzenberg, L.A., et al., Interpreting flow cytometry data: a guide for the perplexed Nature Immunology, 2006. 7(7): p. 681-685.

63. Greve, B., et al., Flow cytometry in cancer stem cells analysis and separation Cytometry Part A, 2012. 81A: p. 284-293.

64. Pappas, D. and K. Wang, Cellular separations: a review of new challenges in analytical chemistry. Analytica Chimica Acta, 2007. 601: p. 26-35. 
65. Herzenberg, L.A., et al., The history and future of the fluorescence activated cell sorter and flow cytometry: a view from stanford. Clinical Chemistry, 2002. 48: p. 1819-1827.

66. Hempel, C.M., K. Sugino, and S.B. Nelson, A manual method for the purification of fluorescently labeled neurons from the mammalian brain. Nature Protocols, 2007. 2(1): p. 2924-2929.

67. Dickson, B. BD FACSJazz Technical Specifications. 2013 [cited 2014 May $24]$ Available from: http://www.bdbiosciences.com/documents/BD_FACSJazz_TechSpecs.pdf.

68. Fernandes, T.G., et al., Stem cell bioprocessing for regenerative medicine. Journal of Chemical Technology and Biotechnology, 2014. 89: p. 34-47.

69. Beitzel, K., et al., Properties of biologic scaffolds and their response to mesenchymal stem cells. Arthroscopy - The Journal of Arthroscopic and Related Surgery, 2014. 30(3): p. 289-298.

70. Choudhery, M.S., et al., Cryopreservation of whole adipose tissue for future use in regenerative medicine. Journal of Surgical Research, 2014. 187(1): p. 24-35.

71. Russell, K.C., et al., Cell-surface expression of neuron-glial antigen 2 (NG2) and melanoma cell adhesion molecule (CD146) in heterogeneous cultures of marrow-derived mesenchymal stem cells Tissue Engineering Part A, 2013. 19(19-20): p. 2253-2266. 
72. Leyva-Leyva, M., et al., Characterization of mesenchymal stem cell subpopulations from human amniotic membrane with dissimilar osteoblastic potential. Stem Cells and Development, 2013. 22(8): p. 1275-1287.

73. Divya, M.S., et al., Umbilical cord blood-derived mesenchymal stem cells consist of a unique population of progenitors co-expressing mesenchymal stem cell and neuronal markers capable of instantaneous neuronal differentiation. Stem Cell Research \& Therapy, 2012. 3: p. 57.

74. Palmon, A., et al., High-efficiency immunomagnetic isolation fo solid tissueoriginated integrin-expressing adult stem cells Methods, 2012. 56: p. 305309.

75. Cizkova, D., et al., Enrichment of rat oligodendrocyte progenitor cells by magnetic cell sorting JOurnal of Neuroscience Methods, 2009. 184: p. 88-94.

76. Rodrigues, G.M.C., et al., Intergrated platform for production and purification of human pluripotent stem cell-derived neural precusors. Stem Cell Review and Reports, 2014. 10: p. 151-161.

77. Vykoukal, J., et al., Enrichment of putative stem cells from adipose tissue using dielectrophoretic field-flow fractionation. Lab on a Chip, 2008. 8: p. 1386-1393.

78. Lu, X., et al., Mesenchymal stem cells from CD34-human umblical cord blood. Transfusion Medicine, 2010. 20: p. 178-184.

79. Bakondi, B. and J.L. Spees, Human CD133-derived bone marrow stromal cells establish ectopic hematopoietic microenvironments in immunodeficient 
mice. Biochemical and Biophysical Research Communications, 2010. 400: p. $212-218$.

80. Masuda, H., et al., A novel marker of human endometrial mesenchymal stemlike cells. Cell Transplantation, 2012. 21: p. 2201-2214.

81. Ahrari, I., et al., CD271 enrichment does not help isolating mesenchymal stromal cells from G-CSF-mobilized peripheral blood. Molecular Biology, 2013. 47: p. 685-691.

82. Iso, Y., et al., Priming with ligands secreted by human stromal progenitor cells promotes grafts of cardiac stem/progenitor cells after myocardial infarction. Stem Cells, 2014. 32: p. 674-683.

83. Wu, A.Y. and D.M. Morrow, Clinical use of dielectrophoresis separation for live adipose derived stem cells Journal of Translational Medicine, 2012. 10: p. 99 .

84. Fong, C.Y., et al., The use of discontinuous density gradients in stem cell research and application. Stem Cell Review and Reports, 2009. 5: p. 428434.

85. Liu, W.H., R. Li, and K.F. Dou, Convenient and efficient enrichment of the CD133+ liver cells from rat fetal liver cells as a source of liver stem/progenitor cells. Stem Cell Review and Reports, 2011. 7(1): p. 94-102.

86. Chen, D.C., et al., Purification of human adipose-derived stem cells from fat tissues using PLGA/silk screen hybrid membranes. Biomaterials, 2014. 35: p. $4278-4287$. 
87. Ito, K., et al., A novel method to isolate mesenchymal stem cells from bone marrow in a closed system using a device made by nonwoven fabric. Tissue Engineering Part C - Methods, 2010. 16: p. 81-91.

88. Federation, I.D. IDF diabetes atlas. 2013 [cited 2014 March 25]; 6:[Available from: http://www.idf.org/diabetesatlas.

89. Weinger, K. and C. Carver, Educating Your Patient with Diabetes. Contemporary Diabetes, ed. A. Veves. 2009, New York: Humana Press.

90. Galadari, S., et al., Role of ceramide in diabetes mellitus: evidence and mechanisms. Lipids in Health and Disease, 2013. 12: p. 98.

91. Yang, X.Y. and S.G. Zheng, Interleukin-22: a likely target for treatment of autoimmune diseases. Autoimmunity Reviews, 2014. 13: p. 615-620.

92. Zinger, A. and G. Leibowitz, Islet transplantation in type 1 diabetes: hype, hope and reality - a clinicians perspective. Diabetes-Metabolsim Research and Reviews, 2014. 30: p. 83-87.

93. Clinic, M. Diseases and conditions: type 1 diabetes. 2014 [cited 2014 May]; Available from: http://www.mayoclinic.org/diseases-conditions/type-1diabetes/basics/definition/con-20019573.

94. Vent-Schmidt, J., et al., The role of FOXP3 in regulating immune responses. International Reviews of Immunology, 2014. 33: p. 110-128.

95. Bacchetta, R., et al., Defective regulatory and effector $T$ cell functions in patients with FOXP3 mutations. Journal of Clincal Investigation, 2006. 116(6): p. 1713-1722. 
96. Bussone, G. and L. Mouthon, Autoimmune manifestations in primary immune deficiencies. Autoimmunity Reviews, 2009. 8(4): p. 332-336.

97. Wildin, R.S., et al., X-linked neonatal diabetes mellitus, enteropathy, and endocrinopathy syndrome is the human equivalent of mouse scurfy. Nature Genetics, 2001. 27(1): p. 18-20.

98. Villasenor, J., C. Benoist, and D. Mathis, AIRE and APECED: molecular insights into an autoimmune disease Immunological Reviews, 2005. 204: p. $156-164$.

99. Undlien, D.E., et al., HLA-encoded genetic predisposition in IDDM - DR4 subtypes may be associated with different degrees of protection. Diabetes, 1997. 46(1): p. 143-149.

100. Bell, G.I., S. Hortia, and J.H. Karam, A polymorphic locus near the human insuline gene is associated with insulin-dependent diabetes-mellitus. Diabetes, 1984. 33(2): p. 176-183.

101. Bottini, N., et al., A functional variant of lymphoid tyrosine phosphastase is associated with type 1 diabetes. Nature Genetics, 2004. 36(4): p. 337-338.

102. Lowe, C.E., et al., Large-scale genetic fine mapping and genotype-phenotype associations implicate polymorphism in the IL2RA region in type 1 diabetes. Nature Genetics, 2007. 39(9): p. 1074-1082.

103. Ueda, H., et al., Associaton of the T-cell regulatory gene CTLA4 with susceptibility to autoimmune disease Nature 2003. 423(6939): p. 506-511. 
104. Bennett, S.T., et al., Susceptibility to human type-1 diabetes at IDDM2 is determined by tandem repeat variation at the insulin gene minisatellite locus. Nature Genetics, 1995. 9(3): p. 284-292.

105. Gamble, D.R., et al., Viral antibodies in diabetes mellitus. British Medical Journal, 1969. 3(5671): p. 627-630.

106. Clements, G.B., D.N. Galbraith, and K.W. Taylor, Coxsackie-B virusinfection and onset of childhood diabetes. Lancet, 1995. 346(8969): p. 221223.

107. Yoon, J.W., T. Onodera, and A.L. Notkins, Virus-induced diabetes-mellitus. $X V$. beta cell damage and insulin-dependent hyperglycemia in mice infected with coxsaxkie virus-B4. Journal of Experimental Medicine, 1978. 148(4): p. 1068-1080.

108. Yoon, J.W., et al., Virus-induced diabetes-mellitus - isolation of a virus from the pancreas of a child with diabetic ketoacidosis. New England Journal of Medicine, 1979. 300(21): p. 1173-1179.

109. Dippe, S.E., et al., Lack of causal association between coxsackie-B4 virusinfection and diabetes. Lancet, 1975. 1(7920): p. 1314-1317.

110. Lonnrot, M., et al., Enterovirus infection as a risk factor for beta-cell autoimmunity in a prospectively observed birth cohort- the finnish diabetes prediction and prevention study Diabetes, 2000. 49(8): p. 1314-1318. 
111. Lonnrot, M., et al., Enterovirus RNA in serum is a risk factor for beta-cell autoimmunity and clincal type 1 diabetes: a prospective study Journal of Medical Virology, 2000. 61(2): p. 214-220.

112. Chatenoud, L., et al., Anti-CD3 antibody induces long-term remission of overt autoimmunity in nonobese diabetic mice Proceedings of the National Academy of Sciences of the United States of America, 1994. 91(1): p. 123127.

113. Pak, C.Y., et al., Association of cytomegalo-virus infection with autoimmune type-1 diabetes. Lancet, 1988. 2(8601): p. 1-4.

114. Zanone, M.M., et al., Association of cytomegalovirus infections with recurrence of humoral and cellular autoimmunity to islet autoantigens and of type 1 diabetes in a pancreas transplanted patient. Transplant International, 2010. 23(3): p. 333-337.

115. Guberski, D.L., et al., Induction of type-1 diabetes by kilham's rat virus in diabetes-resistant BB/WOR rats. Science, 1991. 254(5034): p. 1010-1013.

116. Craig, M., et al., Viruses and type 1 diabetes: a new look at an old story. Pediatric Diabetes, 2013. 14(3): p. 149-158.

117. Van Belle, T.L., K.T. Coppieters, and M.G. Von Herrath, Type 1 diabetes: etiology, immunology, and therapeutic strategies. Physiological Reviews, 2011. 91(1): p. 79-118. 
118. Vaarala, O., M.A. Atkinson, and J. Neu, The "perfect storm" for type 1 diabetes - the complex interplay between intestinal microbiota, gut permeability, and mucosal immunity. Diabetes, 2008. 57(10): p. 2555-2562.

119. Dai, Y.D., et al., Slcllal enhances the autoimmune diabetogenic T-cell response by altering processing and presentation of pancreatic islet antigens. Diabetes, 2009. 58(1): p. 156-164.

120. Malik, F.S. and C.E. Taplin, Insulin therapy in children and adolescents with type 1 diabetes. Pediatric Drugs, 2014. 16(2): p. 141-150.

121. Jain, V., Management of type 1 diabetes in children and adolescents. Indian Journal of Pediatrics, 2014. 81(2): p. 170-177.

122. Pozza, R.D., et al., Diabetes in childhood - cardiovascular consequences. Diabetologie Und Stoffwechsel, 2009. 4(3): p. 177-182.

123. Deli, G., et al., Diabetic neuropathies: diagnosis and management. Neuroendocrinology, 2013.98(4): p. 267-280.

124. Schernthaner, G. and G.H. Schernthaner, Diabetic nephropathy: new approaches for improving glycemic control and reducing risk. Journal of Nephrology, 2013. 26(6): p. 975-985.

125. Cappai, G., et al., Increased prevalence of proliferative retinopathy in patients with type 1 diabetes who are deficient in glucose-6-phosphate dehydrogenase. Diabetologia 2011. 54(6): p. 1539-1542.

126. Brownlee, M., Biochemistry and molecular cell biology of diabetic complications. Nature, 2001. 414: p. 813-820. 
127. Kurra, S., D.A. Fink, and E.S. Siris, Osteoporosis-associated fracture and diabetes. Endocrinology and Metabolism Clinics of North America, 2014. 43(1): p. $233+$.

128. Sunkum, A.J.K. and S. Pingile, A clinical study of audiological profile in diabetes mellitus patients. European Archives of Oto-Rhino-Laryngology, 2013. 270(3): p. 875-879.

129. Secher, A.L., et al., Real-time continuous glucose monitoring as a tool to prevent severe hypoglycaemia in selected pregnant women with type 1 diabetes - an observational study. Diabetic Medicine, 2013. 31(3): p. 352356.

130. Aguayo-Mazzucato, C. and S. Bonner-Weir, Stem cell therapy for type 1 diabetes mellitus. Nature Reviews Endocrinology, 2010. 6(3): p. 139-148.

131. Leeb, C., et al., New perspectives in stem cell research: beyond embryonic stem cells Cell Proliferation, 2011. 44: p. 9-14.

132. Lee, R.H., et al., Multipotent stromal cells from human marrow home to and promote repair of pancreatic islets and renal glomeruli in diabetic NOD/scid mice. PNAS, 2006. 103(103): p. 17438-17443.

133. Ho, J.H., et al., Multiple intravenous transplantations of mesenchymal stem cells effectively restore long-term blood glucose homeostasis by hepatic engraftment and beta-cell differentiation in streptozocin-induced diabetic mice. Cell Transplantation, 2012. 21: p. 997-1009. 
134. Sun, Y., et al., Differentiation of bone marrow-derived mesenchymal stem cells from diabetic patients into insulin-producing cells in vitro. Chinese Medical Journal, 2007. 120(9): p. 771-776.

135. Xie, Q.P., et al., Human bone marrow mesenchymal stem cells differentiate into insulin-producing cells upon microenvironmental manipulation in vitro. Differentiation, 2009. 77(5): p. 483-491.

136. Fiorina, P., J. Voltarelli, and N. Zavazava, Immunological applications of stem cells in type 1 diabetes. Endocrine Reviews, 2011. 32(6): p. 725-754.

137. Volarevic, V., et al., Concise review: mesenchymal stem cell treatment of the complications of diabetes mellitus. Stem Cells 2011. 29(1): p. 5-10.

138. Hisanaga, E., et al., A simple method to induce differentiation of murine bone marrow mesenchymal cells to insulin-producing cells using conophylline and betacellulin-delta4. Endocrine Journal, 2008. 55(3): p. 535-543.

139. Karnieli, O., et al., Generation of insulin-producing cells from human bone marrow mesenchymal stem cells by genetic manipulation. Stem Cells, 2007. 25(1): p. 2837-2844.

140. Li, Y.H., et al., Generation of insulin-producing cells from PDX-1 gene modified human mesenchymal stem cells Journal of Cellular Physiology, 2007. 211(1): p. 36-44.

141. Lu, J., et al., Advancing practical usage of microtechnology: a study of the functional consequences of dielectrophoresis on neural stem cells. Integrative Biology, 2012. 4: p. 1223-1236. 


\section{${ }^{1}$ Chapter 3 Dielectrophoresis Literature Review}

\subsection{Brief Overview: hMSC Separation Shortcomings and Research}

\section{Objectives}

The realization of hMSC-based diabetic therapeutics requires careful characterization of the unique biological and biophysical properties to enable separations of hMSCs from different cells or between hMSC progenitor subpopulations. Dielectrophoretic (DEP) technology is a tool that can be used to characterize cell biophysical properties (membrane capacitance, permittivity, and conductivity). This body of work extends DEP to the exploration of hMSCs. DEP is an ideal candidate for efficient hMSCs separations because it is a rapid, straightforward method capable of purifying desired cell subpopulations from heterogeneous cell populations without changing cell functionality. Because hMSCs are a newly explored cell system, DEP technology can increase understanding of their diverse functions by characterizing the cells dielectric properties - how charges interact with the cell, membrane, cytosol, organelles, and proteins. This work primarily explores charge behaviors around the entire cell and with proteins on the membrane surface. Additionally, an archive that links biosurface markers to hMSC differentiation behaviors has not yet been established. This work is an essential first step in achieving this. Considering all of these attributes, a DEP microfluidic device

\footnotetext{
${ }^{1}$ The material contained in this chapter will be submitted for publication.
} 
has the potential to improve the purification processes for hMSCs therapeutic applications.

Therefore, as a reminder, the objectives of this research are:

Objective 1: Characterize the dielectrophoretic response of hMSCs to determine the DEP spectra including the cross-over frequency.

Objective 1a: Standardize hMSCs morphology with ELP-PEI to reduce size-dependent DEP variations, and characterize the DEP spectra.

Objective 2: Model and calculate hMSCs dielectric properties using MATLAB and the Core-Shell Spherical DEP Polarization models.

Objective 2a: Correlate these properties with known phenotypical molecular expressions of hMSCs.

Objective 2b: Compare dielectric properties with other cell systems.

Objective 3: Develop and optimize a new frequency sweep rate data collection technique for rapid compilation of the frequency dependent DEP spectrum.

Since dielectrophoresis will be used to characterize hMSCs dielectric properties, a review of DEP technology is provided in this chapter. The sections to follow will describe the dielectrophoretic phenomena, length scales and types of cell polarizations, types of DEP, and DEP's current utilization. 


\subsection{Dielectrophoresis Phenomena}

DEP is a separation technique that utilizes nonuniform electric fields to polarize cells based on the dielectric properties of their membrane, cytoplasm, and organelles. The electric fields can be applied with either alternating or direct current (AC or DC), this research utilizes AC electric fields because the applied frequency are controlled easier. Cells will exhibit either positive DEP forces (pDEP), where cells move to areas of high electric field density, or negative DEP forces (nDEP), where cells are repelled from areas of high electric field density. The magnitude of the DEP force can be changed by adjusting the electric field magnitude and shape [13]. Figure 3.1 below illustrates $\mathrm{pDEP}$ and $\mathrm{nDEP}$ in one type of electric field geometry. The DEP force, $\vec{F}_{D E P}$, that a spherical-shaped cell (general case) experiences in an AC field is given by $[1,4]$,

$$
\vec{F}_{D E P}=4 \pi \varepsilon_{\text {med }} \operatorname{Re}\left[f_{C M}\right] R^{3} E_{o} \cdot \nabla \vec{E}_{o}
$$

where $\varepsilon_{\mathrm{med}}$ is the medium permittivity (unitless), $\mathrm{f}_{\mathrm{CM}}$ is the Clausius-Mossotti factor (unitless), $\mathrm{R}^{3}$ is the radius of the cell $(\mu \mathrm{m})$, and $\mathrm{E}_{\mathrm{o}}$ is the electric potential $(\mathrm{V})$.

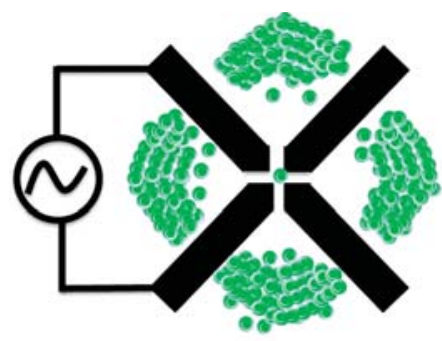

Negative DEP

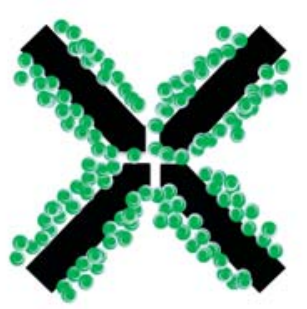

Positive DEP 
Figure 3.1 Schematic of the negative DEP and positive DEP a cell will experience in an $\mathrm{AC}$ electric field with quadrapole electrodes [1-3] .

\subsubsection{Polarization Mechanisms}

There are a variety polarization factors involved with DEP: electronic, atomic, orientational, and interfacial [5]. Consider a cell placed in a conductive medium with an electric field applied. The electric field will interact with the ions available in the medium causing them to move and align around the cells (electronic polarization). On the atomic level the ions present in the conductive medium will move relative to one another (atomic polarization), for example $\mathrm{NaCl}\left(\mathrm{Na}^{+}, \mathrm{Cl}^{-}\right) ; \mathrm{Na}^{+}$ will move relative to $\mathrm{Cl}^{-}$; this movement has previously been assumed to be almost negligible in comparison to the movement associated with the cell in the electric field (however, other research in our group has quantified this motion and shown it can contribute to secondary cell phenomena). Molecules composed of multiple atoms in the conductive medium will also contribute to the polarization induced by the electric field. Water for instance has 3 atoms and a permanent dipole so, in the presence of an electric field, it responds by aligning with the field gradient to reduce potential energy [6].

Lastly, interfacial polarization is a resultant of electronic, atomic, and orientation polarization and is described as ion movement occurring at the interface of two phases, for cells the conductive medium is phase 1 and the cell surface is phase 2. Interfacial polarizations are described by Maxwell Wagner, as charges that 
are built up at the particle-medium interface [7]. This interfacial polarization is characterized by the time is takes for full particle polarization to occur, the dielectric relaxation time, and is given by

$$
\tau_{T W}=\frac{\left(\varepsilon_{p}+2 \varepsilon_{m}\right) \varepsilon_{0}}{\sigma_{p}+2 \sigma_{m}}
$$

where $\varepsilon_{\mathrm{p}}, \varepsilon_{\mathrm{m}}$ are the permittivity of the particle (or cell) and medium respectively, and $\sigma_{\mathrm{p}}, \sigma_{\mathrm{m}}$ is the conductivity of the particle (or cell) and medium, respectively. Any impedance a ion experiences during polarization is due to the dielectric properties of the suspended cell [6].

With AC electric fields applied, cell polarizations occur at specific frequencies and are strongly dependent on cell structure. The cell response to frequencies is also known as the dielectric dispersion with $\alpha$-, $\beta$-, and $\delta$-regions. Since cells have different structures, this dispersion is unique to different cell systems. The $\beta$-dispersion region is important when interrogating the cell's membrane structure, because the electric fields penetrate the cell-conductive medium interface to detect surface features. The $\beta$-dispersion region is characterized by radio frequencies, $0.010-10 \mathrm{MHz}$, and interfacial polarizations are dominant in this region [8]. The other polarization mechanisms are dominant at specific frequency ranges, orientation polarization $0.010-10 \mathrm{MHz}$ and atomic polarization $10^{11}-10^{13} \mathrm{~Hz}$; the polarization length scales are different.

At radio frequencies dipolar and ionic polarizations are significant. Again, take a spherical shaped cell suspended in a conductive medium in a nonuniform 
electric field. The electric field polarizes the cell and induces a moment, and the resultant net force exerted on the cell is represented as the effective moment dotted with the gradient of the electric potential [4],

$$
\vec{F}=\vec{p}_{e f f} \cdot \nabla \vec{E}
$$

Figure 3.2 illustrates the dipole particle with the electric field. The expression for DEP force is obtained by determining the proper expression for $\vec{p}_{\text {eff }}$. The effective moment is determined by solving the spherical electrostatic potential boundary value problem using the proper boundary conditions, which gives [4],

$$
\vec{p}_{e f f}=4 \pi \varepsilon_{1}\left(\frac{\varepsilon_{\text {cell }}-\varepsilon_{\text {med }}}{\varepsilon_{\text {cell }}+2 \varepsilon_{\text {med }}}\right) R^{3} E_{o}
$$

Substituting Equation 3 back into Equation 2 yields the DEP force Equation given in Equation 1.

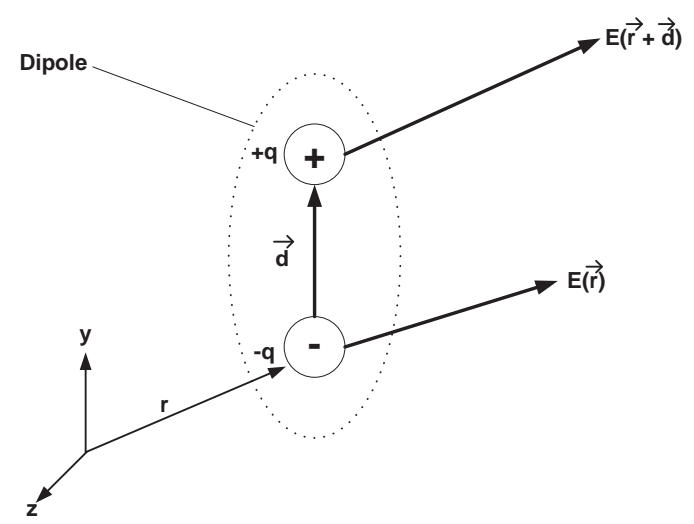

Figure 3.2 Force exerted upon a small dipole by an electric field. Adapted from [4]. 


\subsubsection{Debye Electrical Double Layer}

As a resultant of a cell's polarization an electric double layer of charges are formed at the cell interface also referred to as the Debye or electric double layer (EDL). The EDL contains two layer of charges with potential difference, phase 1 (conductive medium) has a net negative charge and phase 2 (cell) has a net positive charge or vice versa (equivalent to parallel plate capacitance). There are several models established to describe the charge layering phenomena: Hemholtz, GuoyChapma, and Guoy-Chapman-Stern model.

This information is important because during DEP experiments the movement of ions is the key factor to the polarization and the behavior of the ions in the EDL is an indicator of cellular dielectric properties. The dielectric relaxation time is cell-specific and dictates how long it will take for a cell to adequately feel the applied electric field, also critical in cell separations. Also, to state more clearly, the key parameters associated with dielectrophoresis are permittivity and conductivity. These two properties are inversely related and the permittivity can be thought of as a resistance to the surface ion polarization induced by the applied AC electric field. The higher a cell's permittivity the less resistant it is to the AC electric field and the polarization will occur quicker when compared to cells of similar size with smaller permittivity's. Cell size is critical to interfacial polarization, the larger the cell the longer it will take for ions to move around, align, and induce movement, this is evident in Equation 1 for the DEP force. The Clausius-Mossotti factor is the 
indicator in the DEP force equation that is directly correlated to a cell's polarizability because it's dependent on the cell's permittivity and conductivity values.

\subsection{Dielectrophoretic Particle Polarization Models}

\subsubsection{Geometry Overview}

Now, that the different particle polarizations have been discussed let's look at the key equations developed to model the polarization response of cells. A particle's polarization can be modeled using a series of equations dependent on a cells shape, complex permittivity, permittivity, and conductivity. A variety cell shapes can be

modeled using the DEP polarization model equations, and cell complexity can be added to these models by adding layers that account for a cells internal structure (membrane, cytoplasm, nucleus, etc.). The most common cell shapes modeled in this manner are spherical, core-shell sphere, ellipsoid, and core-shell ellipsoid. The hMSCs are our focus, and their main shape is spherical like or star-shaped, which can be modified to spherical via ELP-PEI treatment. Thus, this section will focus on the spherical and core-shell spherical DEP polarization model.

\subsubsection{Homogeneous Sphere DEP Polarization Model}

The homogenous sphere, used for polystyrene beads in Chapter 5, simplifies the cell structure to not contain any internal organelles. For a homogeneous spherical particle, the $f_{C M}$ is given by $[1,4]$, 


$$
\begin{gathered}
f_{C M}=\frac{\widetilde{\varepsilon}_{\text {cell }}-\widetilde{\varepsilon}_{\text {med }}}{\widetilde{\varepsilon}_{\text {cell }}+2 \widetilde{\varepsilon}_{\text {med }}} \\
\widetilde{\varepsilon}_{\text {cell }}=\varepsilon_{\text {cell }}+\frac{\sigma_{\text {cell }}}{j \omega} \\
\widetilde{\varepsilon}_{\text {med }}=\varepsilon_{\text {med }}+\frac{\sigma_{\text {med }}}{j \omega}
\end{gathered}
$$

In Equations $4-6 \varepsilon_{\text {med }}$ is the permittivity of the surrounding medium (unitless), $\operatorname{Re}\left[f_{C M}\right]$ is the real part of the Clausius-Mossotti factor (unitless), $\widetilde{\varepsilon}_{\text {cell }}$ is the complex permittivity of the cell (unitless), $\widetilde{\varepsilon}_{\text {med }}$ is the complex permittivity of the medium (unitless), $\varepsilon_{\text {cell }}$ is the permittivity of the cell (unitless), $\sigma_{\text {cell }}$ is the conductivity of the cell $(\mathrm{S} / \mathrm{m})$, and $\sigma_{m e d}$ is the conductivity of the medium $(\mathrm{S} / \mathrm{m})$. If a particle experiences pDEP, then $f_{C M}$ is positive which indicates that the DEP force displaces the particle in the electric field gradient towards areas of high electric field density. Vice versa is true for a particle experiencing nDEP, $f_{C M}$ is negative and the particle is displaced in the electric field gradient towards areas of low electric field density. When $f_{C M}$ equals zero, cross-over frequency $\left(f_{x o}\right)$, the particle experiences no DEP force $\left(\vec{F}_{D E P}=\mathbf{0}\right)$. The $f_{x o}$ is the point at which a cell (or particle) transitions from experiencing $\mathrm{pDEP}$ to $\mathrm{nDEP}$ or $\mathrm{nDEP}$ to $\mathrm{pDEP}$. The $f_{x o}$ is important because at this transition point the dielectric properties of cells (or particles) can be determined, along with other data points from the DEP spectra [9]. $f_{C M}$ is dependent on the complex permittivity of the cell and the medium (Equation 5-6). If $\underline{\varepsilon}_{\text {med }}>\underline{\varepsilon}_{\text {cell }}$, then $f_{C M}$ will be negative and if the $\underline{\varepsilon}_{c e l l}>\underline{\varepsilon}_{\text {med }}$, then $f_{C M}$ will be positive. The real part of 
the $f_{C M}$ is further dependent on the permittivity and conductivity of the medium and cell, with four cases summarized in Table 3.1. From Equation 1, the cell size plays a role in the DEP force experienced by the cells. As cell size changes the cell DEP force changes.

Table 3.1. High and low frequency examination of the Clausius-Mossotti factor, $f_{C M}$.

\begin{tabular}{|c|c|c|c|c|}
\hline & Case 1 & Case 2 & Case 3 & Case 4 \\
\hline $\begin{array}{l}\text { High } \\
\text { Frequency }\end{array}$ & $\begin{array}{l}\text { If } \sigma_{\text {cell }}>\sigma_{\text {med }} \text {, } \\
\text { then } f_{C M} \text { is } \\
\text { dependent on } \\
\text { permittivity }\end{array}$ & $\begin{array}{l}\text { If } \sigma_{\text {cell }}<\sigma_{\text {med }} \text {, } \\
\text { then } f_{C M} \text { is } \\
\text { dependent on } \\
\text { permittivity }\end{array}$ & $\begin{array}{l}\text { If } \varepsilon_{\text {cell }}> \\
\varepsilon_{\text {med }} \text { then } \\
f_{C M} \text { is } \\
\text { positive }\end{array}$ & $\begin{array}{l}\text { If } \varepsilon_{\text {cell }}< \\
\varepsilon_{\text {med }} \text { then } \\
f_{C M} \text { is } \\
\text { negative }\end{array}$ \\
\hline $\begin{array}{l}\text { Low } \\
\text { Frequency }\end{array}$ & $\begin{array}{l}\text { If } \sigma_{\text {cell }}>\sigma_{\text {med }} \text {, } \\
\text { then } f_{C M} \text { is } \\
\text { positive }\end{array}$ & $\begin{array}{l}\text { If } \sigma_{\text {cell }}<\sigma_{\text {med }} \text {, } \\
\text { then } f_{C M} \text { is } \\
\text { negative }\end{array}$ & $\begin{array}{l}\text { If } \varepsilon_{\text {cell }}> \\
\varepsilon_{\text {med }} \text { then } \\
f_{C M} \text { is } \\
\text { positive }\end{array}$ & $\begin{array}{l}\text { If } \varepsilon_{\text {cell }}< \\
\varepsilon_{\text {med }} \text { then } \\
f_{C M} \text { is } \\
\text { negative }\end{array}$ \\
\hline
\end{tabular}

The permittivity of a cell is a resistance to the electric field, and cells with higher permittivities have less resistance to the electric field and are easier to polarize. This can be thought of time take for ions in a suspending solution take to rearrange around the cells once the AC electric field is applied. The homogeneous sphere does not represent the complexity of a cell, so it's not frequently used to model cell's DEP response. However, the homogeneous sphere of polystyrene beads is useful in evaluating new microdevice designs, and experimental techniques, because PS bead structure is homogeneous. 
The homogeneous spherical DEP polarization model has been used to examine how the $f_{C M}$ changes as a function of frequency at varying medium conductivities. The results of this model are in Figure 3.3, which illustrates that an increase in solution conductivity shift $f_{C M}$, model doesn't account for cell internal organelles.

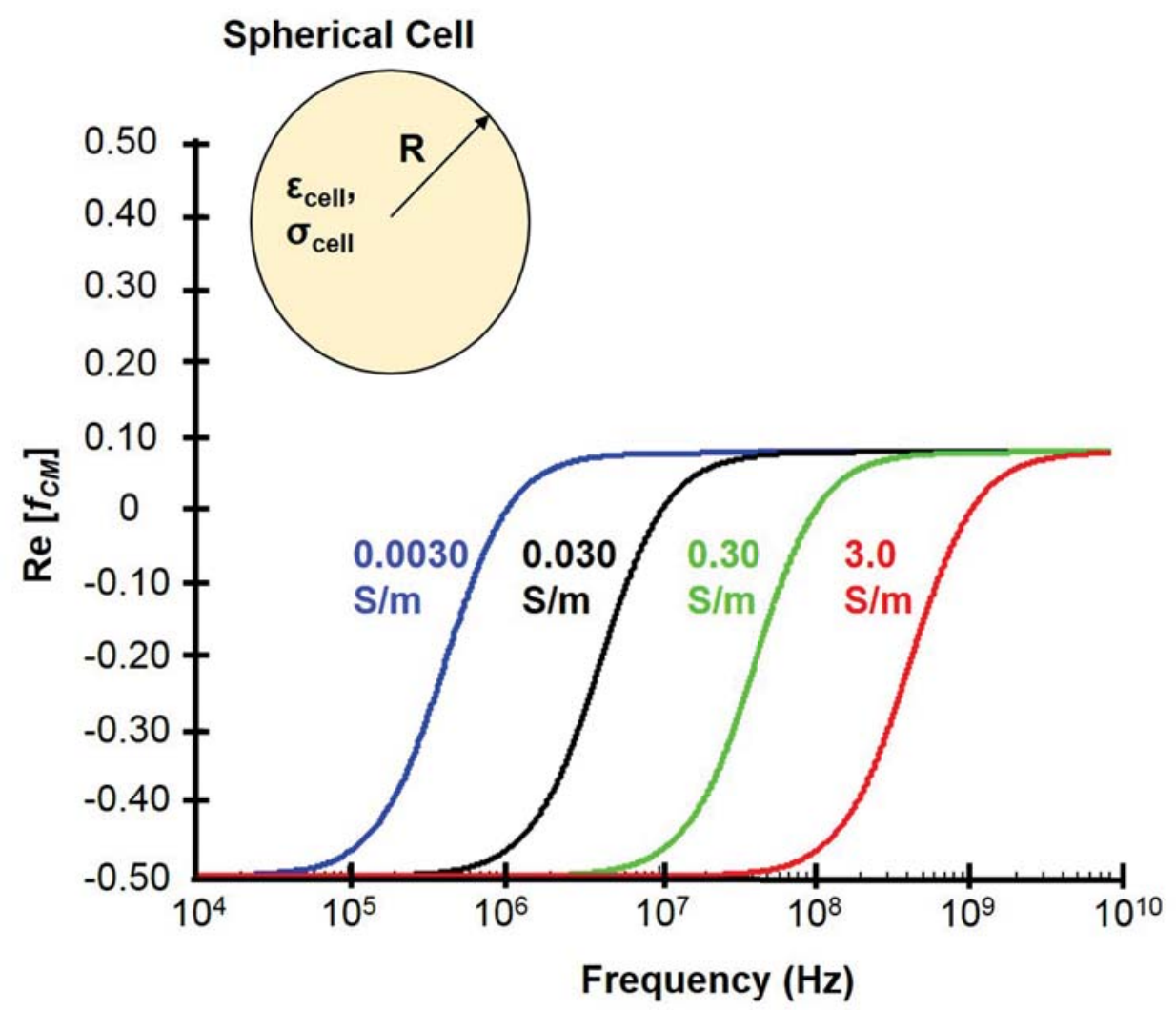

Figure 3.3 Homogeneous sphere DEP polarization models for the $f_{C M}$ of hMSCs in varying conductive mediums. Plot generated in MATLAB using Equations 4-6 [1], where $\varepsilon_{\text {cell }}=100 * \varepsilon_{0}, \varepsilon_{\text {med }}=80^{*} \varepsilon_{0}, \varepsilon_{0}=8.85 * 10^{-12}$, and $\sigma_{\text {cell }}=10^{-6} \mathrm{~S} / \mathrm{m}$. This model does not account for hMSCs internal structure. 


\subsubsection{Core-Shell Spherical DEP Polarization Model}

Because the spherical DEP polarization model does not account for cells structural organelles such as the membrane and cytoplasm, and therefore, it is not an accurate representation of cell's DEP response spectra. A more appropriate model is the core-shell spherical DEP polarization model, which treats cells as a layered particle and accounts for the conductivity and permittivity of both the cell membrane and cytoplasm. Consider a layered spherical cell suspended in a conductive medium in a nonuniform $\mathrm{AC}$ electric field, the DEP force acting on the cell will be the same as in Equation 1. The difference in this case appears in how the $f_{C M}$ is defined because a third region describing the electrostatic potential on the layered cell is added creating an additional boundary condition. Now the complex permittivity of the cell has to account for the complex permittivity of the cell membrane and cytoplasm. This is accomplished by replacing the cell by an equivalent homogeneous sphere defined with the same radius. By solving the new layered spherical electrostatic potential problem the Clausius-Mossotti Factor is now given as [1, 4],

$$
\begin{aligned}
& f_{C M}=\frac{\widetilde{\varepsilon}_{c e l l}^{\prime}-\widetilde{\varepsilon}_{\text {med }}}{\widetilde{\varepsilon}_{\text {cell }}^{\prime}+2 \widetilde{\varepsilon}_{\text {med }}}, \\
& \widetilde{\varepsilon}_{\text {cell }}^{\prime}=\widetilde{\varepsilon}_{\text {med }}\left[\frac{\left(\frac{R_{c y t o}}{R_{c y t o}}\right)^{3}+2\left(\frac{\widetilde{\varepsilon}_{c y t o}-\widetilde{\varepsilon}_{\text {mem }}}{\widetilde{\varepsilon}_{c y t o}+2 \widetilde{\varepsilon}_{\text {mem }}}\right)}{\left.\left(\frac{R_{c y t o}}{R_{c y t o}}\right)^{3}-\left(\frac{\widetilde{\varepsilon}_{c y t o}-\widetilde{\varepsilon}_{\text {mem }}}{\widetilde{\varepsilon}_{c y t o}+2 \widetilde{\varepsilon}_{m e m}}\right)\right],}\right. \\
& \widetilde{\varepsilon}_{c y t o}=\varepsilon_{c y t o}+\frac{\sigma_{c y t o}}{j \omega},
\end{aligned}
$$




$$
\widetilde{\varepsilon}_{m e m}=\varepsilon_{m e m}+\frac{\sigma_{m e m}}{j \omega}
$$

In Equation $7 \underline{\varepsilon}_{\text {cell }}^{\prime}$ is the effective complex permittivity of the cell, which takes into account the complex permittivity of the cytoplasm $\left(\widetilde{\varepsilon}_{\text {cyto }}\right)$ and cell membrane $\left(\widetilde{\varepsilon}_{m e m}\right)$, permittivity of the cytoplasm $\left(\varepsilon_{c y t o}\right)$ and cell membrane $\left(\varepsilon_{\text {mem }}\right)$, as well as the conductivity of the cytoplasm $\left(\sigma_{c y t o}\right)$ and cell membrane $\left(\sigma_{m e m}\right)$. Here the size of the cell plays a very important role and the size is accounted for in the radii. Here is where knowing hMSCs morphology, cell size, and protein expression becomes important, because larger cells will have a different DEP response than smaller cells. The overall goal of this research is to characterize the dielectric behavior of hMSCs and its differentiated progeny.

Figure 3.4 illustrates that for a core-shell sphere a conductivity increase shifts (increases) the $f_{C M}$, similar trend to the homogeneous sphere case. As the cell size changes the cell DEP force changes. The core-shell spherical DEP polarization model was also plotted to show size effects on $f_{C M}$ in a medium with $0.01 \mathrm{~S} / \mathrm{m}$ conductivity, Figure 3.5. In Figure 3.5 as the size of the cell increases $f_{C M}$ increases. 


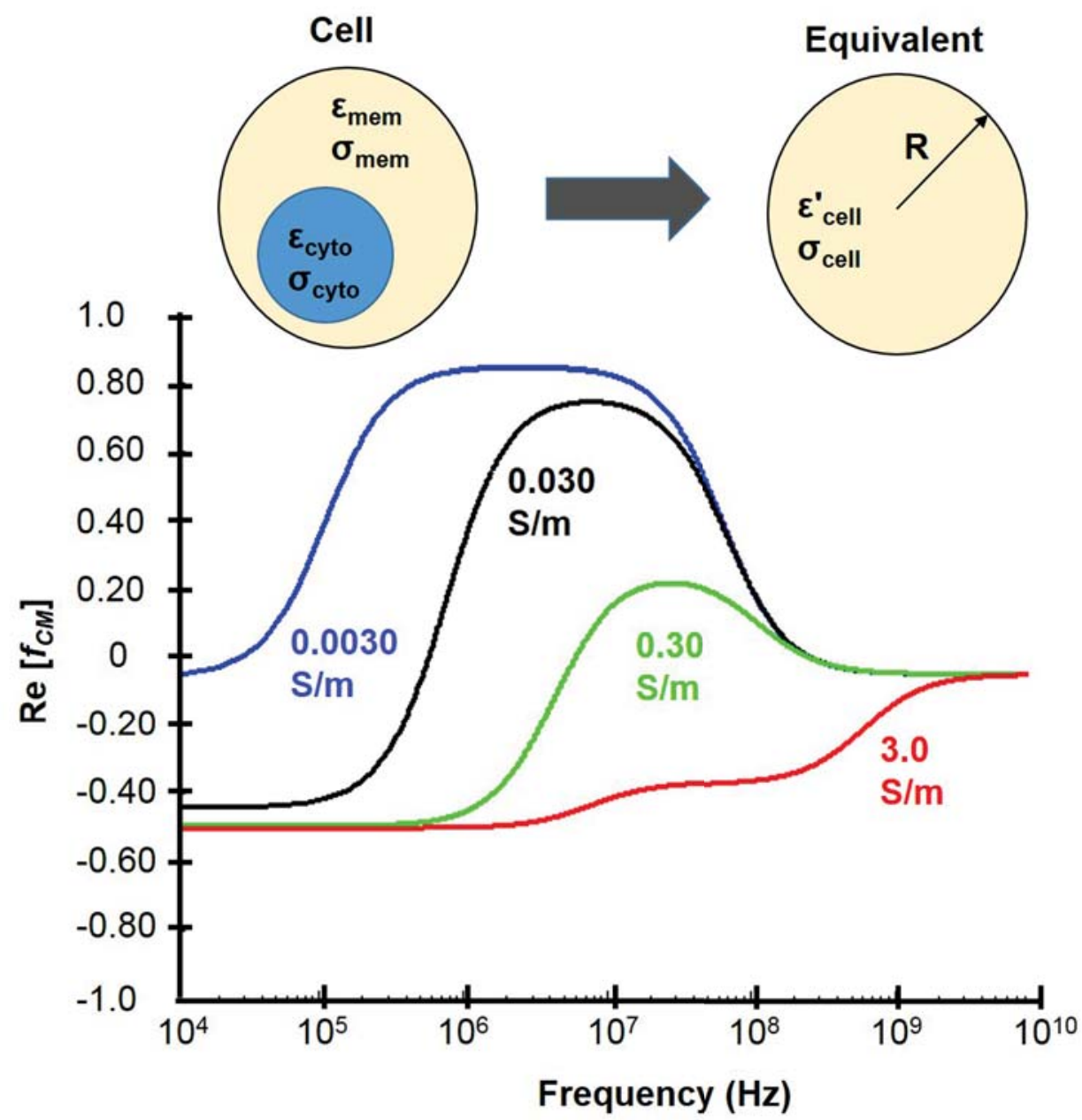

Figure 3.4 Schematic of core-shell spherical cell and equivalent spherical with the core-shell spherical DEP polarization model for hMSCs $f_{C M}$ in varied conductive mediums. Plot generated in MATLAB using Equations 7-10 [1,4] where $\varepsilon_{\text {cyto }}=$ $60 * \varepsilon_{0}, \varepsilon_{\mathrm{mem}}=0.50 * \varepsilon_{0}, \varepsilon_{\mathrm{med}}=80 * \varepsilon_{0}, \varepsilon_{0}=8.85 * 10^{-12}$, and $\sigma_{\mathrm{cyto}}=0.63 \mathrm{~S} / \mathrm{m}, \sigma_{\mathrm{mem}}=10^{-6}$ $\mathrm{S} / \mathrm{m}$. 


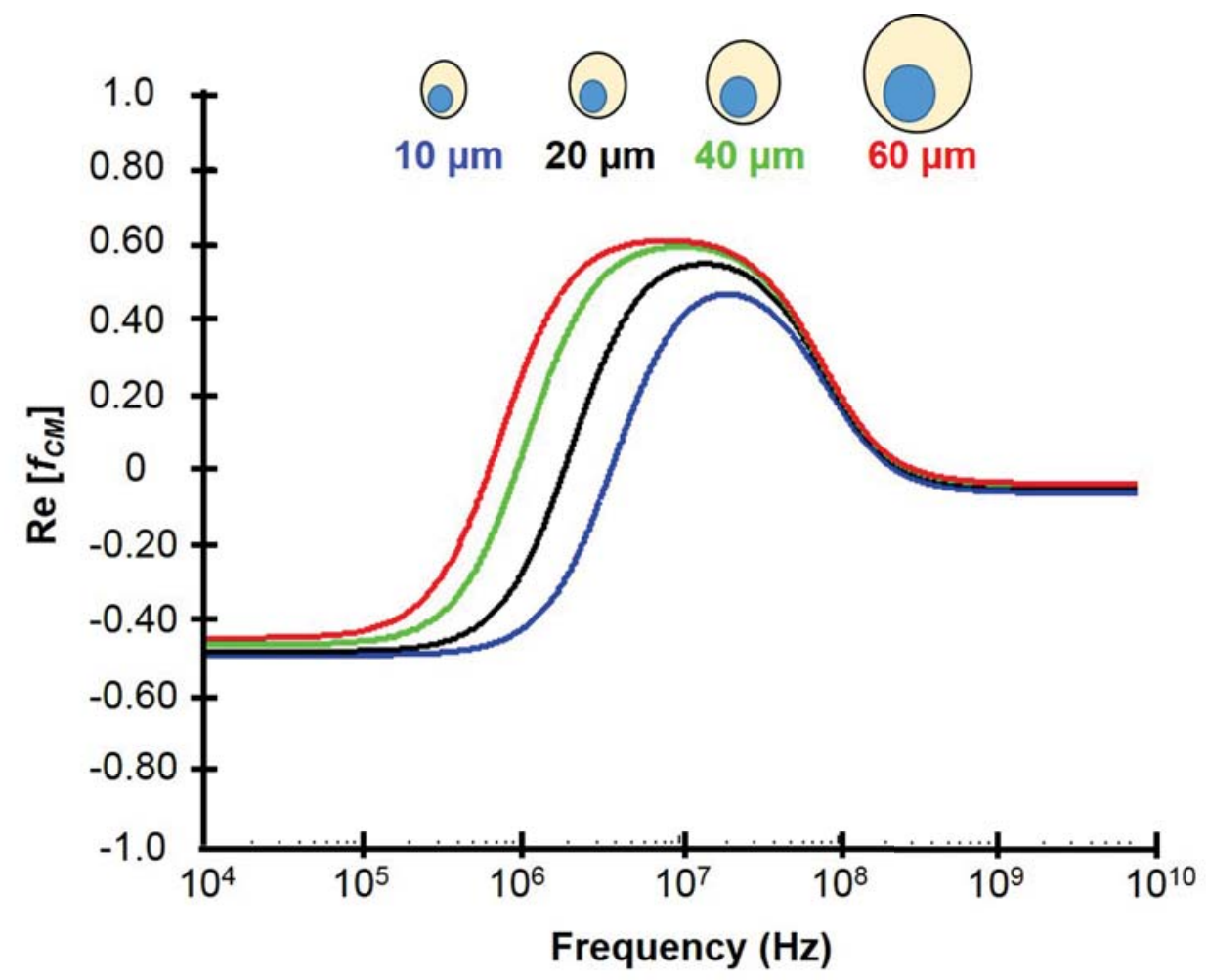

Figure 3.5 Effect of membrane size difference on hMSCs $f_{C M}$ directly correlates to DEP response spectra [5]. Generated using Equations 7-10 in MATLAB where $\varepsilon_{\text {cyto }}$ $=60 * \varepsilon_{0}, \varepsilon_{\mathrm{mem}}=0.50 * \varepsilon_{0}, \varepsilon_{\mathrm{med}}=80^{*} \varepsilon_{0}, \varepsilon_{0}=8.85^{*} 10^{-12}$, and $\sigma_{\mathrm{cyto}}=0.63 \mathrm{~S} / \mathrm{m}, \sigma_{\mathrm{mem}}=$ $10^{-6} \mathrm{~S} / \mathrm{m}$, and $\mathrm{R}=10,20,40,60 \mu \mathrm{m}$.

This modeling is key in the exploration of cells (or particles) because it predicts cellular behavior at specific frequencies. These DEP models can be compared to the experimentally determined DEP response spectra and optimized to determine the dielectric properties membrane/cytoplasm permittivity, conductivity, and membrane capacitance. Once the $f_{x o}$ is determined based on the experimental DEP spectra $f_{x o}$ can be correlated to the membrane capacitance, and then correlated to 
the membrane permittivity. Membrane capacitance, $C_{m e m}$, is a function of $f_{x o}$ and given by $[10,11]$

$$
C_{m e m}=\frac{\sqrt{2} \sigma_{m e d}}{2 \pi r f_{x o}}
$$

where $\mathrm{r}$ is the cell radius $(\mu \mathrm{m})$. Further, the membrane permittivity is proportional to $C_{\text {mem }}$

$$
\varepsilon_{m e m}=\frac{C_{m e m} d}{4 \pi r^{2} \varepsilon_{0}},
$$

where $d$ is the membrane thickness and $\varepsilon_{0}$ is the vacuum permittivity. Therefore, the $f_{x o}$ and $C_{m e m}$ can be used as indicators of treatment-induced hMSC changes and can be lumped into $\varepsilon_{m e m}$, a key dielectric property. To obtain the dielectric properties, the membrane and cytoplasm conductivity and permttivity values are adjusted until good aggreement is made between experimental data and theory predictions. The experimentally determined $f_{x o}$ is used to estimate the experimental membrane capacitance.

Modeling hMSCs as core-shell spherical particles is a more accurate representation of their complexity but is not $100 \%$ accurate. There are morphological variations within an hMSC population ( as mentioned in Chapter 2), and in order to have reproducible DEP responses as well as detect biosurface protein markers these variations need to be removed from the DEP measurement. To achieve this, elastinlike polypeptide (ELP) polyethyleneimine (PEI) was employed as a standardization technique and Chapter 4 reports results. 
This research uses a quadraple microelectrode design in order to achieve DEP motion within experiments. There are many other microelectrodes possible, and they will be briefly discussed in the next section.

\subsection{Types of Dielectrophoresis Electrode Designs}

Different types of DEP are characterized by the electrode design, and specific design utilization are dependent on the application necessary. Traditional DEP is classified by common electrode designs implemented into experimental studies, which are interdigitated, castellated, curved and quadrapole; insulator/contactless DEP is alternative method to traditional DEP and it prevents cells from coming in contact with operational electrodes, and lastly traveling wave DEP is used from cell/fluid pumping. This section will overview these designs in more details and show their current use in research.

\subsubsection{Traditional DEP}

Traditional DEP uses a couple of different electrode configurations. For each design type the electrodes are patterned along the bottom of a microchannel or microchamber [12]. These designs include: interdigitated, castellated, curved, and quadrapole (design used in this research); this list in not all inclusive. Figure 3.6 (ad) illustrates these different electrode designs. The electrode design selection is made based on specific functions needed. Traditional DEP electrode designs are typically used for cell separations or enrichment. Each design shapes the electric field gradient 
differently, and the position where the gradient is highest is marked with a red circle in Figure 3.6 (a-d). The advantages to using these designs are that they are fabricated easily, and require low electric fields to induce cell movement, which is critical to maintain cell viability. The disadvantage to these designs are that they expose cells to AC electric fields which means the electrode material used has to be biocompatible [12]. Typical electrode materials used are gold and titanium [8].

(a) Interdigitated

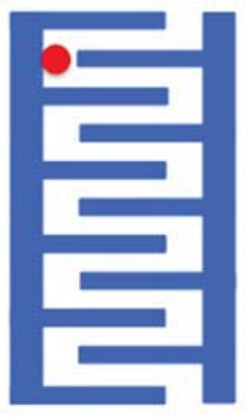

(b) Castellated

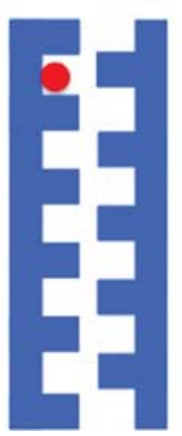

(c) Curved

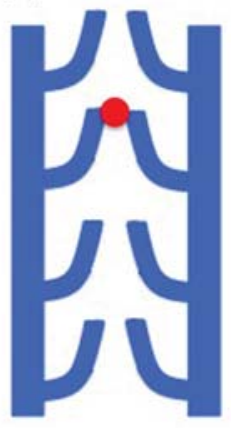

(d) Quadrapole

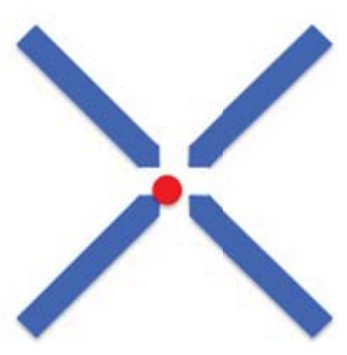

Figure 3.6 Common traditional DEP electrode designs (a) interdigitated, (b) castellated, (c) curved, (d) quadrapole. Each device design is used for cell trapping based on cell dielectric properties. Adapted from [12].

Interdigitated, castellated, curved, and quadrapole electrodes are all used for cell trapping either in the high electric field gradient area, $\mathrm{pDEP}$, or in the low field electric field gradient area, nDEP. Table 3.2 summarizes the applications these electrode designs have been used in. 
Table 3.2 Summary of electrode configuration applications.

\begin{tabular}{|c|c|c|c|}
\hline Configuration & Particle & Application & Ref. \\
\hline \multirow[t]{4}{*}{ Interdigitated } & $\begin{array}{l}\text { Prostate circulating } \\
\text { tumor cells }\end{array}$ & $\begin{array}{l}\text { DEP immunocapture } \\
\text { system }\end{array}$ & [13] \\
\hline & $\begin{array}{l}\text { Human neonatal dermal } \\
\text { fibroblast and mouse } \\
\text { embryonic stem cells }\end{array}$ & $\begin{array}{l}\text { Estimation of } \\
\text { dielectric properties } \\
\text { based on \% trapping }\end{array}$ & [14] \\
\hline & Viral nano-lysate & HIV virus detection & [15] \\
\hline & $\begin{array}{l}3 \mathrm{~T} 3 \text { and embryonic stem } \\
\text { cells }\end{array}$ & $\begin{array}{l}\text { DEP-based cell } \\
\text { pairing }\end{array}$ & [16] \\
\hline \multirow[t]{4}{*}{ Castellated } & Latex nanoparticles & $\begin{array}{l}\text { DEP image } \\
\text { processing and } \\
\text { dielectric property } \\
\text { measurement }\end{array}$ & [17] \\
\hline & $\begin{array}{l}\text { Murine embryonic stem } \\
\text { cells }\end{array}$ & $\begin{array}{l}\text { Embryoid formation } \\
\text { for stem cell } \\
\text { differentiation }\end{array}$ & [18] \\
\hline & $\begin{array}{l}\text { Mycobacterium } \\
\text { smegmatis bacterial cells }\end{array}$ & $\begin{array}{l}\text { Alive dead cell } \\
\text { isolation }\end{array}$ & [19] \\
\hline & Porcine oocytes & $\begin{array}{l}\text { Healthy oocyte } \\
\text { selection for in vitro } \\
\text { fertilization }\end{array}$ & [20] \\
\hline \multirow[t]{3}{*}{ Curved } & $\begin{array}{l}\text { Tungsten trioxide and } \\
\text { polystyrene nanoparticles }\end{array}$ & $\begin{array}{l}\text { Particle concentration } \\
\text { mapping }\end{array}$ & [21] \\
\hline & Yeast cells & $\begin{array}{l}\text { Live and dead cell } \\
\text { sorter }\end{array}$ & [22] \\
\hline & Latex microbeads & $\begin{array}{l}\text { microdevice utility } \\
\text { for continuous cell } \\
\text { sorting }\end{array}$ & [23] \\
\hline \multirow[t]{4}{*}{ Quadrapole } & $\begin{array}{l}\text { Polystyrene beads and } \\
\text { red blood cells }\end{array}$ & $\begin{array}{l}\text { Frequency sweep rate } \\
\text { data collection utility }\end{array}$ & [8] \\
\hline & Red blood cells & Blood typing & [24] \\
\hline & Colloidal crystals & $\begin{array}{l}\text { Colloidal assembly } \\
\text { manipulations }\end{array}$ & [25] \\
\hline & HeLa cells & $\begin{array}{l}\text { AC electrothermal } \\
\text { flow trapping }\end{array}$ & {$[26]$} \\
\hline
\end{tabular}




\subsubsection{Insulator/contactless DEP (iDEP or cDEP)}

Another type of DEP separation is insulator DEP also known as contactless DEP (cDEP), this method uses insulating postings within the microfluidic channel to assist separations and the electrodes are located along the edges of the microdevice, Figure 3.7. Specifically, the electrodes are isolated from the main microfluidic channel by a thin membrane [27], and polyermic posts are placed in the channel to distort the field creating a gradient [28]. The posts can have varied geometries such as diamonds, circles, and triangles. DEP force equation and Clausius-Mossotti factor remain the same with these setups.

Some applications for iDEP/cDEP are ABO-Rh blood typing [29], separation of yeast cell and polystyrene bead mixture [30], P. aeruginosa and S. mitis bacteria strain level discrimination [31], particle focusing [32], and high performance particle trapping on a silicon substrate [33].

The advantages to this method are simple fabrication, DEP force is distributed over large area (increases throughput), low $\mathrm{AC}$ fields used for separations, and single cell analysis. A disadvantages is the possibility of joule heating [12], applied AC field releases heat increasing system temperature [34]. 
(a)

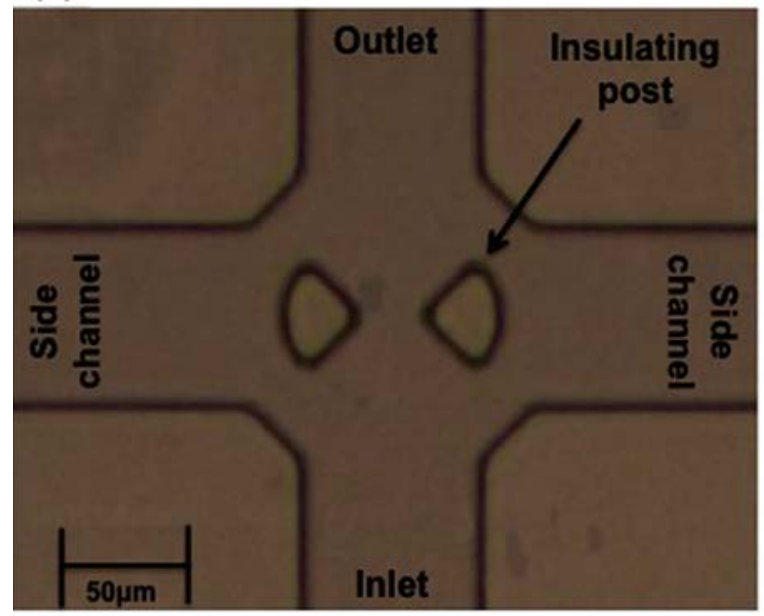

(c)

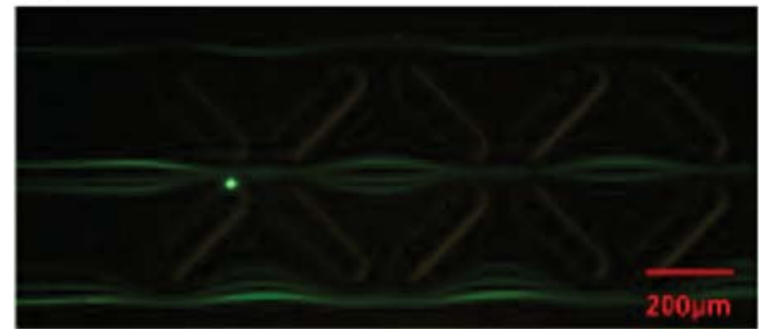

(b)

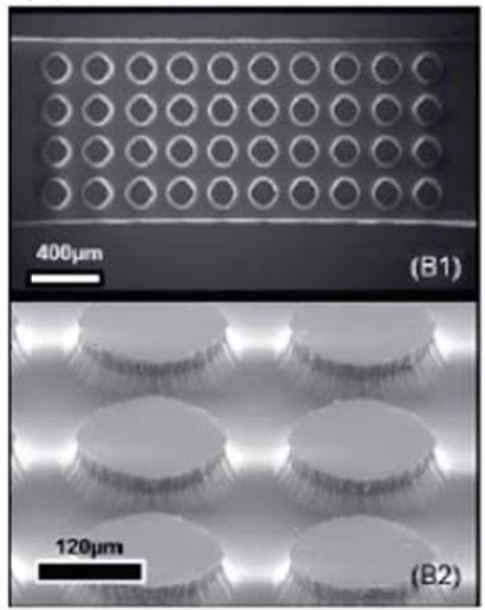

(d)

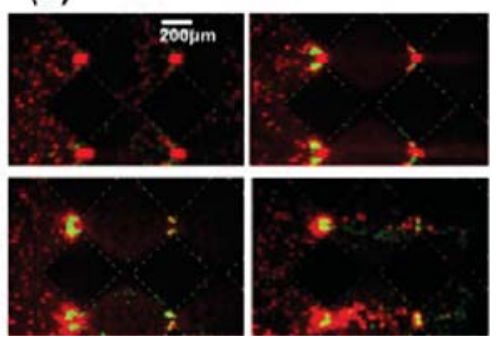

Figure 3.7 Varied insulating posts design inside iDEP microdevices. (a) rounded triangluar [27], (b) circular [33], (c) elongated [32], and (d) triangular [30]. Reproduced with permission from [27, 30, 32, 33].

\subsubsection{Traveling Wave DEP (twDEP)}

Pumping is another operation strategies that can be implemented using DEP with a slightly different electrode setup consisting of rectangular parallel electrode array pattern, Figure 3.8. This electrode design facilitates horizontal particle movement in a nonuniform electric field, and the electric field gradient maxima occurs at the center of the electrodes therefore cells travels in wave down the center 
of the electrodes. This electrode design functions by turning on two electrodes at a specific frequency, and then turning one electrode off and stepping to the next electrode (turning on the next electrode). This causes the electric field gradient to move or travel down the electrodes, hence the name traveling wave DEP (twDEP). The time-average DEP force equation is now dependent on the real and imaginary part of the Clausius-Mossotti factor [35],

$$
\left\langle F_{D E P}\right\rangle=2 \pi \varepsilon_{\text {med }} R^{3}\left\{\operatorname{Re}\left[f_{C M}\right] \nabla E^{2}+\operatorname{Im}\left[f_{C M}\right] \sum E^{2} \nabla \phi\right\}
$$

The velocity of the cells in the electric field is critical and specific to cell being examined, different dielectric properties affects cell movement or mobility. Velocity is dependent on $f_{C M}$ and is given by [35],

$$
v_{D E P}=\frac{R^{2} \varepsilon_{r} \varepsilon_{0} \operatorname{Re}\left[f_{C M}\right]}{3 \eta} \nabla E
$$

twDEP has been used as an alternative to FACS and MACs cell sorting method by van den Driesche et al., in their work they separated jurkat cells from with S. cervisiae (yeast) and Lactobacillus casei (bacteria found in the mouth and intestine). The separation was achieved by first determining a separation frequency $\mathrm{f}_{\mathrm{xo}}$ for jurkat cells using a quadrapole electrode design and choosing a device operation frequency such that target cells experienced a small nDEP force away from the electrodes. The twDEP was applied perpendicular to the flow, so that the untargeted bacteria were adhered to the electrodes. Hydrodynamic flow allowed the targeted jurkat cells to be collected [36]. Figure 3.8 illustrates the device setup and real images of cell separation. 


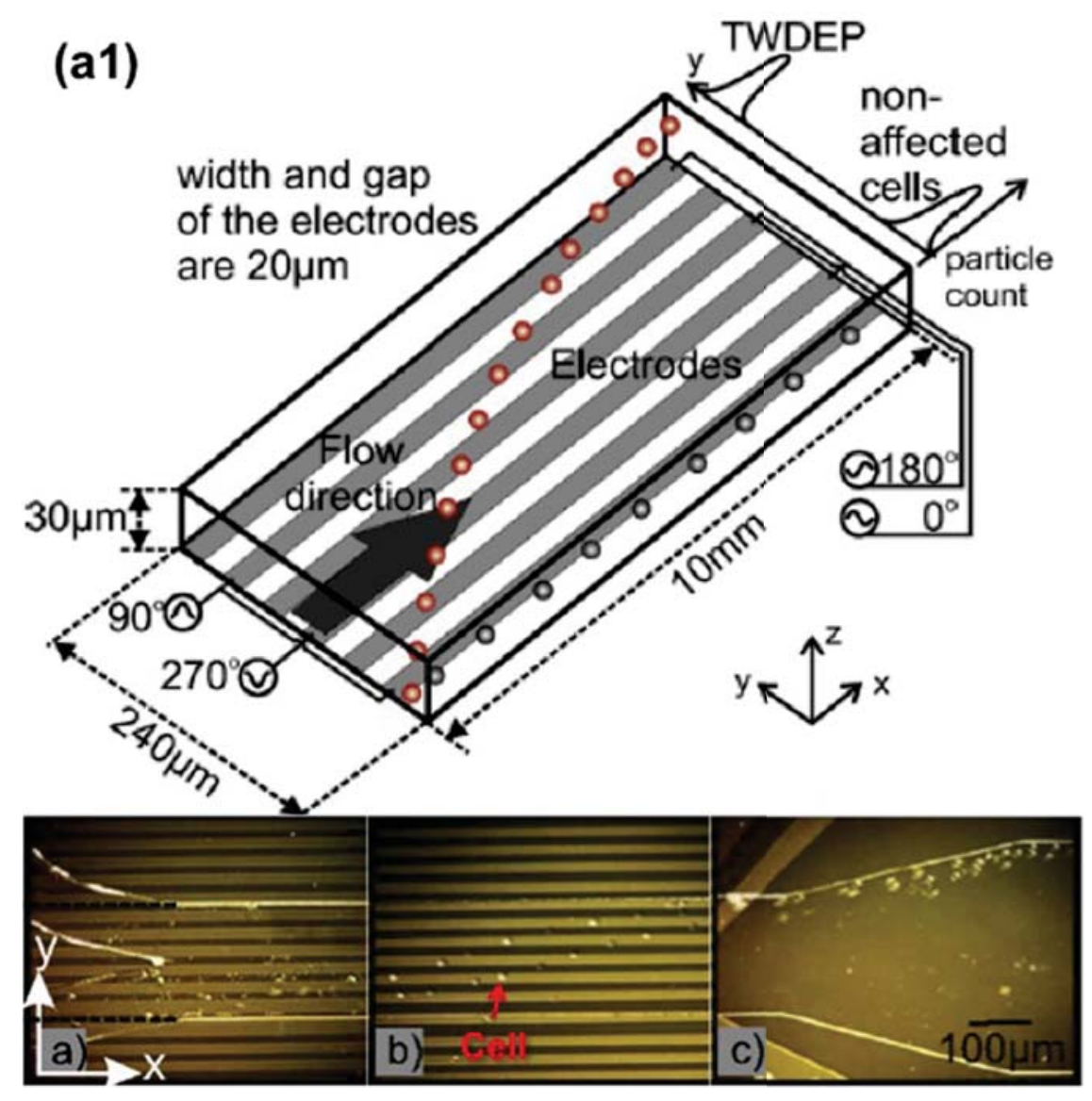

Figure 3.8 twDEP separation of jurkat cells from S. cervisiae and Lactobacillus casei bacteria. Jurkat cells were separated by flowing along the twDEP field gradient with $\mathrm{nDEP}$ force and the bacteria cells were adhered to the electrodes with $\mathrm{pDEP}$ force. Reprint produced with permission from [36].

The main applications of twDEP is to pump cells for sorting and other cell systems be explored using this technique are red blood cells [37], yeast, and microparticles [38]. Each method discussed above traditional DEP, iDEP/cDEP, and twDEP are useful in particle manipulation and determining the dielectric properties of biological cells. In this dissertation the quadrapole electrode design is used due to 
is simple fabrication process. The quadrapole electrode DEP microdevice used in this work is easily networked with syringe pumps, a function generator, and microscope to complete experimental tests.

\subsection{Microdevice Dielectrophoretic Applications}

\subsubsection{General Applications and Stem Cell Studies}

Dielectrophoretic technology has been around for awhile with the earliest work occurring in 1974 completed by Chen and Pohl. In this work single yeast cells were exploited in nonuniform electric fields created with platinum wires. Individual cell characteristics, permittivity and age, were tabulated using voltage release response. The effective polarizability varied from cell to cell, but variations were reproducible. The voltage response spectrum was able to detect variability within the yeast cell population indicative of subpopulations (similar to hMSCs). This dielectric response varied with cell age condition, dead cell excess permittivity decreased, parameters may be useful in cell studies [5]. This study is at the core of all other DEP studies to follow over the next 40 years.

Studies have now transformed to look at many animal and human cell lineages. In a study completed by Muratore et al., myoblast $\mathrm{C} 2 \mathrm{C} 12$ cells were examined because of their similarities to stem cells (i.e. they differentiate). This work detected different membrane capacitance signatures for fibroblasts, differentiated $\mathrm{C} 2 \mathrm{C} 12$, and undifferentiated $\mathrm{C} 2 \mathrm{C} 12$ cells using a symmetrical electrode design that funneled cells during sorting to the outlet, Figure 3.9a. The 
cells were tested in $0.12 \mathrm{~S} / \mathrm{m}$ conductive medium, and the membrane capacitance difference between the undifferentiated $\mathrm{C} 2 \mathrm{C} 12$ and fibroblast cells were attributed to surface microvilli, with SEM images adding supporting evidence, Figure 3.9b [39].

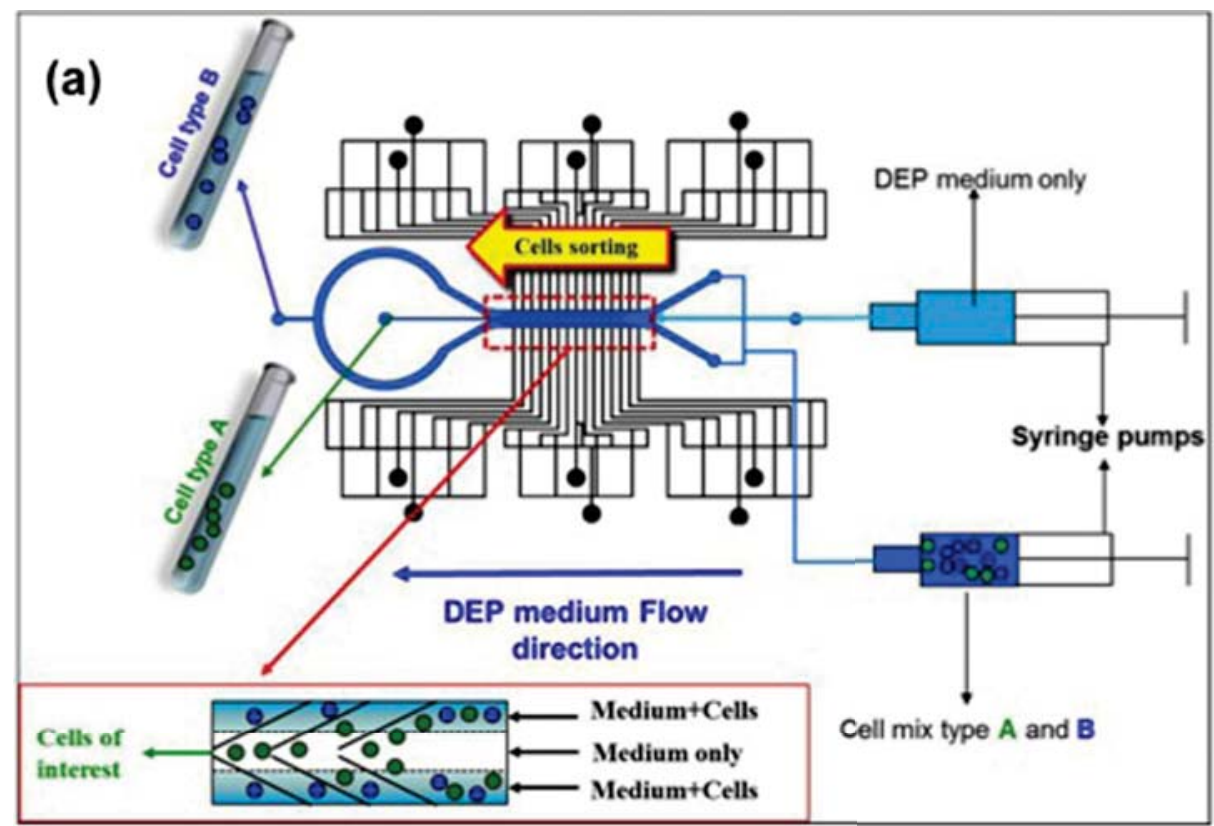

(b)

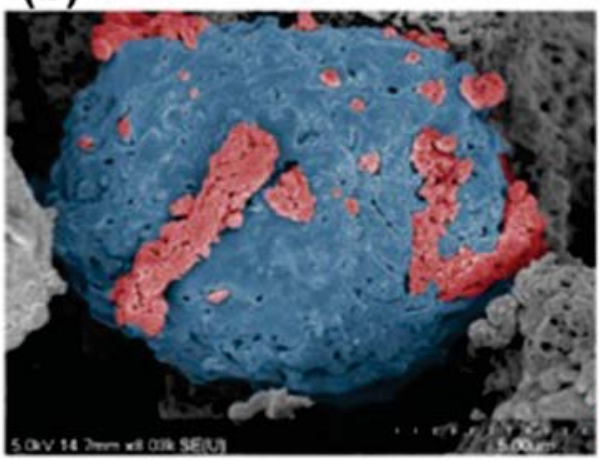

(c)

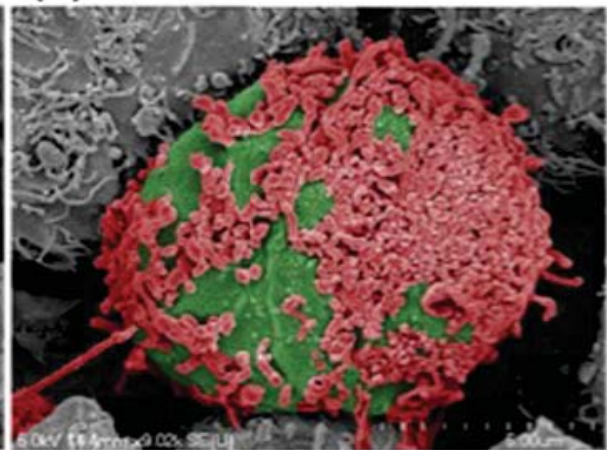

Figure 3.9 Shows the cell sorting microdevice and corresponding SEM images of sorted fibroblasts and undifferentiated $\mathrm{C} 2 \mathrm{C} 12$ cells. Reproduced with permission from editor [39]. 
Another DEP study by Duncan et al., explored the $\mathrm{Cl}^{-}, \mathrm{K}^{+}$, and $\mathrm{Ca}^{+2}$ ionic contributions to the cytoplasm conductivity of drug resistant and nondrug resistant leukemic cells (K562 and K562AR, respectively). K562 and K562AR were treated with verapamil (calcium channel blocker), quinine (potassium channel blocker), and NPPB (chloride channel blocker) and the cells spatial variation were tabulated in the presence of $\mathrm{AC}$ electric fields at $0.0025 \mathrm{~S} / \mathrm{m}$. The channel blocker treatments altered K562 and K562AR DEP response indicating reductions in their cytoplasm conductivity. As a result $\mathrm{Cl}^{-}, \mathrm{K}^{+}$, and $\mathrm{Ca}^{+2}$ ionic contributions to $\mathrm{K} 562$ 's and K562AR's cytoplasmic conductivity were found to be $\mathrm{K} 562: 0.13 \mathrm{~S} / \mathrm{m} \mathrm{K}^{+}, 0.08 \mathrm{~S} / \mathrm{m}$ $\mathrm{Ca}^{+2}$, and $0.09 \mathrm{~S} / \mathrm{m} \mathrm{Cl}^{-}$; K562AR: 0.16S/m K, $0.08 \mathrm{~S} / \mathrm{m} \mathrm{Ca}^{+2}$, and $0.25 \mathrm{~S} / \mathrm{m} \mathrm{Cl}^{-}$. Based on the ionic contributions it can be inferred that the physiological difference between K562 and K562AR is associated with the presence of extra chlorides (increased chloride channel activity). This work demonstrates that DEP is a good analysis tool to detect ion channel activity within cells [40].

DEP is also a good research tool for stem cells studies. In some of the first stem cell DEP research it was demonstrated that hematopoietic stem cells could be concentrated from a mixed cell population without requiring cell manipulation [41, 42]. Adipose-derived putative stem cells were also concentrated by coupling DEP and field-flow fractionation (FFF) [41, 43]. Flanagan et al., examined the dielectric properties of mouse neural stem/precursor cells (NSPCs) using parallel electrodes (50 $\mu \mathrm{m}$ in width with $100 \mu \mathrm{m}$ gaps) with an $\mathrm{AC}$ electric field. Figure 3.10 below shows an image of the real microfluidic device used. In this study the NSPCs were 
suspended in a sucrose buffer solution with a conductivity of $150 \mu \mathrm{S} / \mathrm{cm}$ and injected in a microchannel. An electric potential of $8 \mathrm{~V}$ was applied and the frequency was varied from $25 \mathrm{kHz}$ to $25 \mathrm{MHz}$ using a function generator. The DEP response of the cells was recorded in 10 to 15 second videos and analyzed. The results of this study show that NSPCs, neurons, and astrocytes have unique dielectric properties that were detected in the system in Figure 3.10. These unique dielectric properties were detected by looking at the percent of cells trapped at specific frequency, trapped cell are those attracted to the electrodes or positive DEP [44]. These results are given in Figure 3.11. The work completed by Flanagan et al., did not calculate the dielectric properties of NSPCs, and there was no connection made with the to the biochemistry aspect of the stem cells.

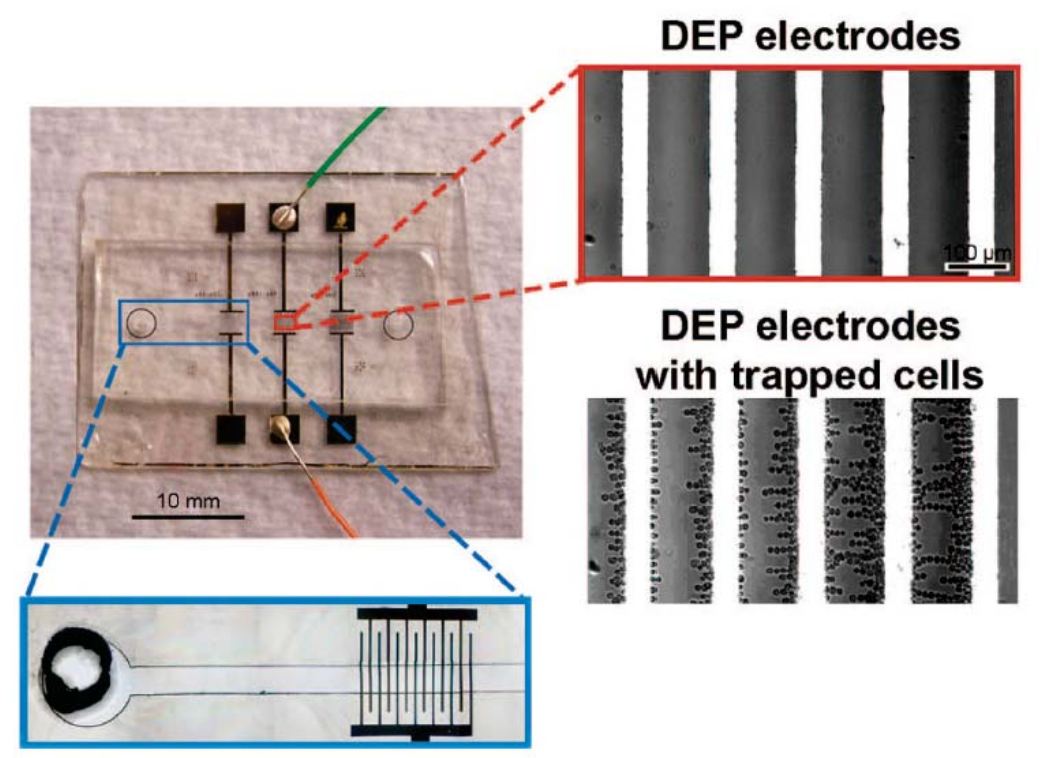

Figure 3.10 Real image of the parallel electrode microfluidic device used to examine the dielectric behavior of NSPCs. Reproduced with permission from editor [44]. 


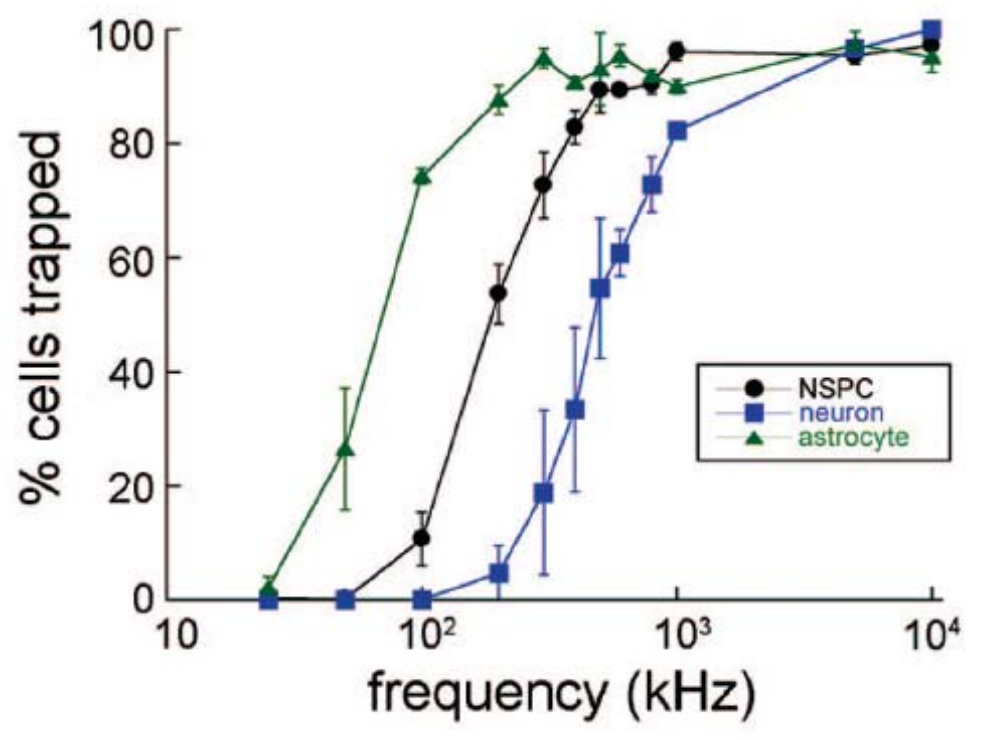

Figure 3.11 Percent trappings results for NSPCs, neurons, and astrocytes. Each cell type has distinct percentage of cells trapped at particular frequencies, which is an indication of unique dielectric properties among each cell type. Reproduced with permission from editor [44].

DEP is a versatile technology and has been implemented in other studies looking at the effects of DEP treated hydrogen peroxide on enamel teeth whitening [45], pesticide detection [46], water quality testing [47, 48], and DNA electrical property determination [49]. 


\subsubsection{DEP Studies to Determine Cell Membrane Capacitance}

A cell's DEP spectra can be correlated to its membrane capacitance, which is an indicator of biosurface proteins. This is of particular interests in hMSCs DEP separations because the biosurface proteins are attributed to hMSCs subpopulations. hMSCs membrane capacitance up to this point has not been explored (we accomplish this in chapter 4), so the membrane capacitance of other cell systems will be discussed in this section.

Table 3.3 summarizes the cell membrane capacitance of neural precursor/stem progenitor cells (NSPCs) with neurogenic progenitor (NP) and astrogenic progenitors (AP); embryonic (E12.5 or E16.5) mouse NSPCs; multiple lineages of oral squamous carcinoma cells (CaLH3, H357, OSCC1, and DOK); adipose-derived stem cells (ADSCs); adipogenic progenitors; osteogenic progenitors; and ADSCs differentiated to osteoblasts. These cell systems were tested in varied conductivity solutions ranging from $0.01 \mathrm{~S} / \mathrm{m}$ to greater than $1 \mathrm{~S} / \mathrm{m}$ (cell culture media). The oral squamous carcinoma cells were characterized based on subpopulations identified by their adhesiveness: rapid adherent cells (RAC), middle adherent cells (MAC), and late adherent cells (LAC). Each of these adhesive subpopulations were correlated to tumorigenic capability with RAC $>$ MAC $>$ LAC [50], which indicates that DEP can be used to detect the tumor cells from healthy cells, useful for cancer therapeutics. Lastly, the measured membrane capacitance for each cell system is consistent with theory, higher capacitance values are 
experimentally determined in higher conductivity mediums due to their proportional relationship (Equation 11).

Table 3.3 Summary of cell membrane capacitance studies using DEP.

\begin{tabular}{|c|c|c|c|c|}
\hline \multicolumn{2}{|c|}{ Cell Type } & $\begin{array}{c}\text { Conductivity } \\
(\mathrm{S} / \mathrm{m})\end{array}$ & $\begin{array}{c}\mathrm{C}_{\text {mem }} \\
(\mathrm{pF})\end{array}$ & Ref \\
\hline \multicolumn{2}{|c|}{ NSPCs-NP biased } & 0.01 & 3.3 & {$[51]$} \\
\hline \multicolumn{2}{|c|}{ NSPCs-AP biased } & 0.01 & 4.3 & [51] \\
\hline \multicolumn{2}{|c|}{ E12.5 mNSPCs } & 0.01 & 1.6 & [51] \\
\hline \multicolumn{2}{|c|}{ E16.5 mNSPCs } & 0.01 & 2.8 & {$[51]$} \\
\hline \multicolumn{2}{|c|}{$\begin{array}{l}\text { SW Treated-E16.5 } \\
\text { mNSPCs }\end{array}$} & 0.01 & 4 & {$[51]$} \\
\hline CaLH3 & $\begin{array}{c}\text { RAC } \\
\text { MAC } \\
\text { LAC }\end{array}$ & 0.10 & $\begin{array}{l}4.1 \\
3.4 \\
2.9\end{array}$ & {$[50]$} \\
\hline H357 & $\begin{array}{l}\text { RAC } \\
\text { MAC } \\
\text { LAC }\end{array}$ & 0.10 & $\begin{array}{l}3.3 \\
2.9 \\
2.3\end{array}$ & {$[50]$} \\
\hline OSCC1 & $\begin{array}{l}\text { RAC } \\
\text { MAC } \\
\text { LAC }\end{array}$ & 0.10 & $\begin{array}{l}1.6 \\
1.3 \\
1.1 \\
\end{array}$ & {$[50]$} \\
\hline DOK & $\begin{array}{l}\text { RAC } \\
\text { MAC } \\
\text { LAC }\end{array}$ & 0.10 & $\begin{array}{l}5.1 \\
5.1 \\
5.8\end{array}$ & [50] \\
\hline \multicolumn{2}{|l|}{ ADSC } & $>1.0$ & 6.6 & {$[52]$} \\
\hline \multicolumn{2}{|c|}{$\begin{array}{l}\text { Adipogenic- } \\
\text { progenitor }\end{array}$} & $>1.0$ & 9.0 & {$[52]$} \\
\hline \multicolumn{2}{|c|}{$\begin{array}{l}\text { Osteogenic- } \\
\text { progenitor }\end{array}$} & $>1.0$ & 6.9 & {$[52]$} \\
\hline \multicolumn{2}{|c|}{$\begin{array}{l}\text { Differentiated } \\
\text { osteoblasts }\end{array}$} & $>1.0$ & 5.7 & {$[52]$} \\
\hline
\end{tabular}

To review, the advantages to using density centrifugation paired with FACS and MACS for hMSC separation are high selectively, automated, commercial availability, and continuous processes [53]. The disadvantages of these processes are that long process times, high cost, large electronic systems, and the antigen labeling 
necessary to achieve target cell isolation [54]. A cell separation system that can improve upon these disadvantages will provide an alternative method for hMSC separation, which may be more suitable for hMSC clinical therapeutics.

With that being said the advantages of using DEP microdevices over FACS and MACs systems are the potential for low cost (microdevices can be mass produced), electric fields are nonharmful to biological cells [55], quick separation times (less than 5mins) [8], decreased resources necessary to achieve separations ( $\mu 1$ sample volumes) [7], and the versatility within microdevices [56]. In a FACS or MACS system the only procedure that can be accomplished is cell separation based on biosurface markers (ideal). DEP microdevices gives cell separations added versatility such as further bioprocessing like cell culturing [57], lysis [58], microreactor chambers for differentiation [59], and viability screening [60]. These steps can all be concurrently combined on a single microfluidic platform.

\subsection{Conclusions}

DEP is a versatile analytical technique that has been employed to study biological cell systems. At the foundation of DEP technology is electronic polarization that occurs due to ion movement around a cell, Maxwell-Wagner interfacial polarization dominant at radio frequencies. Cells response to the ion movement is not instantaneous and dielectric relaxation time is required for the cell to respond to ion movement. This time is cell specific and was utilized in the design of a new DEP data collection technique in chapter 5. 
Additionally, the core-shell spherical DEP polarization model provides adequate predictions of hMSCs DEP response (Chapter 4). The DEP spectra for cells is dependent on cell size, dielectric properties, and medium conductivity. Cells suspended in a higher medium conductivity will experience a shift in the DEP spectra due to the increased number of ions present in the suspending solution. The dielectric properties can be extracted from the DEP response spectra because the membrane is directly related to $f_{\mathrm{xo}}$ and the entire DEP spectra. A variety of electrode designs are available for DEP experiments but the design type used is dependent on the function required by the microdevice. Our work uses the quadrapole electrode design because its easy to fabricate. Many particle/cell systems have been studied with DEP technology jurkat cells, leukemic cells, and mouse neural precursor/stem progenitor cells, just to name a few. However, hMSCs have not been studied with DEP, therefore this dissertation work extends DEP technology to this novel cell system for separations that can be utilized for type 1 diabetes therapeutics.

\subsection{References}

1. Kirby, B.J., Micro- and Nanoscale Fluid Mechanics: Transport in Microfluidic Devices. 2010, New York: Cambridge University Press.

2. Zhang, C., Khoshmanesh, K., and Mitchell, A., Dielectrophoresis for manipulation of micro/nano particles in microfluidic systems. Analytical and Bioanalytical Chemistry, 2010. 396: p. 401-420. 
3. Hawkins, B.G., Huang, C., Arasanipalai, S., and Kirby, B.J., Automated dielectrophoresis characterization of mycobacterium smegmatis. Analytical Chemistry, 2011. 83: p. 3507-3515.

4. Jones, T.B., Electromechanics of particles. 2005, New York: Cambridge University Press.

5. Chen, C.S. and H.A. Pohl, Biological dielectrophoresis: the behavior of lone cells in a nonuniform electric field. Annals of the New York Academy of Sciences, 1974. 238: p. 176-185.

6. Pohl, H.A., Dielectrophoresis: The behavior of neutral matter in nonuniform electric fields. 1978, Cambridge: Cambridge University Press.

7. Pethig, R., Review Article-Dielectrophoresis: Status of the theory, technology, and applications. Biomicrofluidics, 2010. 4(2): p. 35.

8. Adams, T.N.G., K.M. Leonard, and A.R. Minerick, Frequency sweep rate dependence on the dielectrophoretic response of polysytrene beads and red blood cells Biomicrofluidics, 2013. 7: p. 064114.

9. Gagnon, Z.R., Cellular dielectrophoresis: Applications to the characterization, manipulation, separation, and patterning of cells.

Electrophoresis, 2011. 32: p. 2466-2487.

10. Salmanzadeh, A., et al., Sphingolipid metabolites modulate dielectric characteristics of cells in a mouse ovarian cancer progression model. Integrative Biology, 2013. 5: p. 843-852. 
11. Gascoyne, P.R.C., et al., Correlations between the dielectric properties and exterior morphology of cells revealed by dielectrophoretic field-flow fractionation. Electrophoresis, 2013. 34: p. 1042-1050.

12. Khoshmanesh, K., et al., Dielectrophoretic platforms for bio-microfluidic systems. Biosensors \& Bioelectronics, 2011. 26(5): p. 1800-1814.

13. Huang, C., et al., Characterization of a hybrid dielectrophoresis and immunocapture microfluidic system for cancer cell capture. Electrophoresis, 2013. 34: p. 2970-2979.

14. Rozitsky, L., et al., Quantifying continuous-flow dielectrophoretic trapping of cells and micro-particles on micro-electrode array. Biomedical Microdevices, 2013. 15: p. 859-865.

15. Shafiee, H., et al., Acute on-chip HIV detection through label-free electrical sensing of viral nano-lysate. Small, 2013. 9: p. 2553-2563.

16. Sen, M., et al., Cell pairing using a dielectrophoresis-based device with interdigitated array electrodes Lab on a Chip, 2013. 13: p. 3650-3652.

17. Bakewell, D.J., J. Bailey, and D. Holmes, Advancing image quantification methods and tools for analysis of nanoparticle electrokinetics. AIP Advances, 2013. 3: p. 102101.

18. Agarwal, S., et al., Formation fo embryoid bodies using dielectrophoresis. Biomicrofluidics, 2012. 6: p. 024101. 
19. Zhu, K., et al., Separation by dielectrophoresis of dormant and nondormant bacterial cells of Mycobacterium smegmatis. Biomicrofluidics, 2010. 4: $\mathrm{p}$. 022809 .

20. Choi, W., et al., Dielectrophoretic oocyte selection chi for in vitro fertilization. Biomedical Microdevices, 2008. 10: p. 337-345.

21. Chrimes, A.F., et al., Dielectrophoresis-Raman spectroscopy system for analysing suspended nanoparticles Lab on a Chip, 2011. 11: p. 921-928.

22. Khoshmanesh, K., et al., Dielectrophoretic-activated cell sorter based on curved microelectrodes. Microfluidics Nanofluidics, 2010. 9: p. 411-426.

23. Khoshmanesh, K., et al., Dielectrophoretic manipulation and separation of microparticles using curved microelectrodes. Electrophoresis, 2009. 30: p. $3707-3717$.

24. Leonard, K.M. and A.R. Minerick, Explorations of ABO-Rh antigen expressions on erythrocyte dielectrophoresis: Changes in cross-over frequency. Electrophoresis, 2011. 32(18): p. 2512-2522.

25. Edwards, T.D., D.J. Beltran-Villegas, and M.A. Bevan, Size dependent thermodynamics and kinetics in electric field mediated colloidal crystal assembly. Soft Matter, 2013. 9: p. 9208-9218.

26. Jang, L.S., P.H. Huang, and K.C. Lan, Single-cell trapping utilizing negative dielectrophoretic quadrupole and microwell electrodes. Biosensors \& Bioelectronics, 2009. 24: p. 3637-3644. 
27. Bhattacharya, S., et al., Selective trapping of single mammalian breast cancer cells by insulator-based dielectrophoresis. Analytical and Bioanalytical Chemistry, 2014. 406: p. 1855-1865.

28. Simmons, B.A., et al., The development of polymeric devices as dielectrphoretic separators and concentrators. MRS Bulletin, 2006. 31: p. $120-124$.

29. Jubery, T.Z., S.K. Srivastava, and P. Dutta, Dielectrophoretic separation of bioparticles in microdevices: a review. Electrophoresis, 2014. 35: p. 691-713.

30. Gencoglu, A., et al., Dynamic microparticle manipulation with an electroosmotic flow gradient in low-frequency alternating current dielectrophoresis Electrophoresis, 2014. 35: p. 362-373.

31. Braff, W.A., et al., Dielectrophoresis-based discrimination of bacteria at the strain level based on their surface properties. PLOS One, 2013. 8: p. e76751.

32. Jen, C.P., et al., Particle formation in a contactless dielectrophoretic microfluidic chip with insulating structures. Microsystems Technology, 2012. 18: p. 1879-1886.

33. Zellner, P. and M. Agah, Silicon insulator-based dielectrophoresis devices for minimized heating effects. Electrophoresis, 2012. 33: p. 2498-2507.

34. Kale, A., et al., Joule heating effects on reservoir-based dielectrophoresis. Electrophoresis, 2014. 35: p. 721-727.

35. Adams, T., et al., A tunable microfluidic device for drug delivery, in Advances in Microfluidics. 2012. 
36. van den Driesche, S., et al., Continuous cell from cell separation by traveling wave dielectrophoresis Sensors and Actuators B, 2012. 170: p. 207-214.

37. Lei, U., et al., A traveling wave dielectrophoretic pump for blood delivery. Lab on a Chip, 2009. 9: p. 1349-1356.

38. Eguchi, M., H. Imasato, and T. Yamakawa, Particle separation by employing non-uniform electric fields, traveling-wave electric fields and inclined gravity. Intelligent Automation and Soft Computing, 2012. 18: p. 121-137.

39. Muratore, M., S. Mitchell, and M. Waterfall, Plasma membrane characterization, by scanning electron-microscopy, of multipotent myoblastsderived populations sorted using dielectrophoresis. Biochemical and Biophysical Research Communications, 2013. 438: p. 666-672.

40. Duncan, L., et al., Dielectrophoretic analysis of changes in cytoplasmic ion levels due to ion channel blocker action reveals underlying differences between drug-sensitive and multidrug-resistant leukemic cells. Physics in Medicine and Biology, 2007. 53: p. N1-N7.

41. Pethig, R., Review article - Dielectrophoresis: Status of the theory, technology, and applications. Biomicrofluidics, 2010. 4: p. 022811.

42. Pethig, R., Menachery, A., Pells, S., and De Sousa, P., Dielectrophoresis: A review of applications for stem cell research. Journal of Biomedicine and Biotechnology, 2010. 2010.

43. Vykoukal, J., Vykoukal, D.M., Freyberg, S., Alt, E.U., and Gascoyne, P.R.C., Enrichment of putative stem cells from adipose tissue using 
dielectrophorestice field-flow fractionation. Lab on a Chip, 2008. 8: p. 13861393.

44. Flanagan, L.A., Lu, J., Wang, L., Marchenko, S.A., Jeon, N.L., Lee, A.P., and Monuki, E., Unique dielectric properties distinguish stem cells and their differentiated progeny. Stem Cells, 2008. 26: p. 656-665.

45. Ivanoff, C.S., et al., Dielectrophoresis enhances the whitening effects of carbamide peroxide on enamel. American Journal of Denistry, 2011. 24: p. 259-263.

46. Ramon-Azcon, J., et al., Detection fo pestide residues using an immunodevice based on negative dielectrophoresis. Biosensors \& Bioelectronics, 2009. 24 : p. $1592-1597$.

47. Hubner, Y., K.F. Hoettges, and M.P. Hughes, Water quality test based on dielectrophoretic measurements of fresh water algae Selenastrum capricornutum. Journal of Environmental Monitoring, 2003. 5: p. 861-864.

48. Betts, W.B. and A.P. Brown, Dielectrophoretic analysis of microbes in water. Journal of Applied Microbiology, 1999. 85: p. 201S-213S.

49. Mohamad, A.S., J.C.G. Jeynes, and M.P. Hughes, Dielectrophoretic response of DNA shows different conduction mechanisms for Poly $(d G)$-Poly $(d C)$ and Poly(dA)-Poly(dT) in solution. IEEE Transactions on Nanobioscience, 2014. 13: p. $51-54$. 
50. Liang, X., et al., Human oral cancer cells with increasing tumorigenic abilities exhibit higher effective membrane capacitance. Integrative Biology, 2014. 6: p. 545-554.

51. Nourse, J.L., et al., Membrane biophysics define neuron and astrocyte progenitors in the neural lineage. Stem Cells, 2014. 32: p. 706-716.

52. Bagnaninchi, P.O. and N. Drummond, Real-time label-free monitoring of adipose-derived stem cell differentiation with electric cell-substrated impedance sensing. PNAS, 2011. 108: p. 6462-6467.

53. Gonzalez-Gonzalez, M., et al., Current strategies and challenges for the purification of stem cells. Journal of Chemical Technology and Biotechnology, 2012. 87: p. 2-10.

54. Diogo, M.M., C. Lobato de Silva, and J.M.S. Cabral, Separation technologies for stem cell bioprocessing. Biotechnology and Bioengineering, 2012. 109: p. 2699-2709.

55. Lu, J., et al., Advancing practical usage of microtechnology: a study of the functional consequences of dielectrophoresis on neural stem cells. Integrative Biology, 2012. 4: p. 1223-1236.

56. Gagnon, Z.R., Cellular dielectrophoresis: Applications to the characterization, manipulation, separation and patterning of cells. Electrophoresis, 2011. 32(18): p. 2466-2487.

57. Kim, D., et al., Microfluidics-based in vivo mimetic systems for the study of cellular biology. Accounts of Chemical Research, 2014. 47: p. 1165-1173. 
58. Ameri, S.K., et al., All electric approach for high-throughput cell trapping and lysis with electrical impedance monitoring. Biosensors \& Bioelectronics, 2014. 54: p. 462-467.

59. Tanaka, Y., et al., Design and fabrication of a dielectrophoresis-based cellpositioning and cell-culture device for construction of cell networks.

Microchemical Journal, 2009. 91: p. 232-238.

60. Patel, S., et al., Microfluidic separation of live and dead yeast cells using reservoir-based dielectrophoresis. Biomicrofluidics, 2012. 6: p. 034102. 


\section{Chapter 4 Characterizing the Dielectric Properties of}

Human Mesenchymal Stem Cells and the Effects of Charged Elastin-like Polypeptide Copolymer Treatment [1]

1 T.N.G. Adams, P.A. Turner, A.V. Janorkar, F. Zhao, and A.R. Minerick

\subsection{Abstract}

Human mesenchymal stem cells (hMSCs) have three key properties that make them desirable for stem cell therapeutics: differentiation capacity, trophic activity, and ability to self-renew. However, current separation techniques are inefficient, time consuming, expensive and in some cases alter hMSCs cellular function and viability. Dielectrophoresis (DEP) is a technique that uses alternating current (AC) electric fields to spatially separate biological cells based on the dielectric properties of their membrane and cytoplasm. This work implements the first steps toward the development of a continuous cell sorting microfluidic device by characterizing native hMSCs dielectric signatures and comparing them to hMSCs morphologically standardized with a polymer. A quadrapole Ti-Au electrode microdevice was used to quantify the dielectric properties based on the DEP frequency spectra and cross-over frequency of hMSCs from $0.010-35 \mathrm{MHz}$ in dextrose buffer solutions $(0.030 \mathrm{~S} / \mathrm{m}$ and

1 The material contained in this chapter was previously published in Biomicrofluidics, 2014. 8: p. 054109. 
$0.10 \mathrm{~S} / \mathrm{m})$. The membrane capacitance and permittivity were found to be $2.2 \mathrm{pF}$ and 2.0 in $0.030 \mathrm{~S} / \mathrm{m}$ and $4.5 \mathrm{pF}$ and 4.1 in $0.10 \mathrm{~S} / \mathrm{m}$, respectively. Elastin-like polypeptide (ELP-) polyethyleneimine (PEI) copolymer was used to control hMSCs morphology to spheroidal cells and aggregates. Results demonstrated that ELP-PEI treatment controlled hMSCs morphology, increased experiment reproducibility, and concurrently increased hMSCs membrane permittivity to shift the cross-over frequency above $35 \mathrm{MHz}$. Therefore, ELP-PEI treatment may serve as a tool for the eventual determination of biosurface marker-dependent DEP signatures and hMSCs purification.

Key Words: Dielectrophoresis, human mesenchymal stem cells, elastin-like polypeptide polyethyleneimine, cross-over frequency, membrane capacitance, membrane permittivity

Common abbreviations: Human Mesenchymal Stem Cells (hMSCs), Dielectrophoresis (DEP), Alternating Current (AC), Elastin-like polypeptide (ELP), Polyethyleneimine (PEI), positive dielectrophoresis (pDEP), negative dielectrophoresis (pDEP) 


\subsection{Introduction}

Human mesenchymal stem cells (hMSCs) are an interesting cell source to researchers because of their regenerative $[1,2]$ and immunological properties $[3,4]$. hMSCs are isolated from the bone marrow and other locations in the body [5] due to their beneficial properties. They have a high differentiation capacity (adipocytes, chondrocytes, osteoblasts, etc.) [6-10], self-renew [11, 12], and secrete bioactive molecules (trophic activity) $[2,3,13,14]$. hMSCs trophic activity is substantial to their function because signals are sent to surrounding cells triggering tissue repair [13] causing apoptosis inhibition, proliferation, and matrix production [13, 14]. hMSCs are being pursued as a therapeutic option for many chronic diseases such as lupus [15], diabetes mellitus [16], cardiomyopathy [17], liver cirrhosis [18], and Crohn's disease [19].

For therapeutic treatments, obtaining hMSCs is a multistep process. hMSCs are a heterogeneous population $[20,21]$ therefore following bone marrow isolation they are centrifuged via density gradient solution (step 1), adhered to plastic cell culture dish (step 2), and separated using trypsinization (step 3) [17]. This method is inefficient and time consuming [22], so other techniques are employed, fluorescentand magnetic-activated cell sorting (FACS and MACS), which implement unique cellsurface antigens or other recognition elements to tag target cells. This 'labeling' of cells alters cellular function, which is not desirable [22-24]. FACS and MACS require expensive raw materials and are labor intensive. There is not a unique biosurface marker that distinguishes hMSCs from other cell populations [21, 25], the minimum 
requirements established by the International Society for Cellular Therapy are: (1) plastic adherence (generic cellular property [6]); (2) positive expression of CD105, CD73, and CD90; (3) negative expression for CD34, CD45, CD11a, CD19, CD14, and HLA-DR; and (4) adipogenic, osteogenic, and chondrogenic differentiation potential $[8,16]$. hMSCs have differing biosurface marker expressions on their membrane [26] making it difficult to establish a unique biosurface marker for characterization.

Additionally, hMSCs morphology has variations, which can create subpopulations [27]. Therefore a label-free, one-step cell purification technique that rapidly purifies hMSCs by accounting for their morphology without altering cellular function is needed.

Dielectrophoresis (DEP) is a separation technique that has potential to overcome the shortcomings of density gradient centrifugation, FACS, and MACS and could provide an electrical biomarker for hMSCs. DEP technologies enable a variety of particle polarizations with nonuniform AC electric fields on microchips $[28,29]$ to achieve particle manipulation. DEP has been used to study other cell systems such as red blood cells [30, 31], cancer cells [32, 33], white blood cells [34], and yeasts cells $[35,36]$. The red blood cell DEP studies are important because different ABO-Rh surface antigens were distinguishable from the DEP spectra. The long-term goals of characterizing hMSCs dielectric properties are to discern unique biosurface markers specific to hMSC subcultures. 
Advances have been made in the study of stem cells using dielectrophoresis. Flanagan et al. [37], looked at the affects AC electric field expsosure times have on cell viabilty. They found that for human and mouse neural stem/progenitor cells short exposure times (30s-1min) between $0.010-10 \mathrm{MHz}$ did not affect cell viability and

metabolic activity. However, longer exposure times, 5-30mins, at the same frequencies induced 20 to $40 \%$ decrease in cell viability and metabolic activity. These results establish experimental parameters, and aligns with cell viability results found in this work after $90 \mathrm{~s} \mathrm{AC}$ field exposure hMSC cell viability remained intact. Wu and Morrow [38] conducted a one patient clinical study on stromal vascular fraction (SVF) cells separated via DEP. The SVF cells were obtained, separated at $15 \mathrm{Vpp}, 0.10$ $1.0 \mathrm{MHz}$ and autologously transplanted to a patients hand to treat muscle atrophy. Positive results were achieved indicating that DEP treated cells were not harmful and they accelerated the healing process over 3 months (as compared to control). The advantages to coupling DEP with microfluidics are microliter sample size, quick analysis ( $\sim$ minutes to achieve results), little sample preparation, and minimal waste production. Disadvantages are that extended electric field exposure times ( $>5 \mathrm{mins})$ negatively affect cells properties and viability [37].

\subsection{Background}

DEP utilizes nonuniform electric fields for cell movement based on the polarizability and dielectric properties (permittivity and conductivity) of their membrane, cytoplasm, and other structurally dominant organelles [39]. Cells have 
distinct dielectric dispersions that can be used as an identification tool for cell purification. A cell's complex permittivity is frequency dependent and characterized by the $\alpha, \beta$, and $\gamma$ dielectric dispersion regions $\left(\omega_{\alpha}<\omega_{\beta}<\omega_{\gamma}\right)[40]$. At radio frequencies ( $\beta$-region), $0.010-10 \mathrm{MHz}$, the dielectric dispersion of cells are affected by their membrane; high frequencies penetrate a cells surface and interogates the internal structure. Therefore, a plethora of information can be obtained about a cell population in the $\beta$-region; many researchers complete their experiments within this frequency range.

In the $\beta$-region, Maxwell-Wagner interfacial polarizations dominate the DEP response phenomena. Based on permittivity and conductivity, polarized cells will exhibit either positive DEP (pDEP), cells move to areas of high electric field gradient, or negative DEP (nDEP) force; cells move to areas of low electric field gradient [41, 42]. The DEP force is given by, $F_{D E P}=2 \pi \varepsilon_{\text {med }} R_{\text {mem }}^{3} \operatorname{Re}\left[f_{C M}\right] \nabla E^{2}$ and this cell motion in the electric field is defined by the Clausius-Mossotti factor, $\mathrm{f}_{\mathrm{CM}}$, for core-shell spherical particles [39, 41]

$$
\begin{aligned}
& f_{C M}=\frac{\tilde{\varepsilon}_{c e l l}^{\prime}-\tilde{\varepsilon}_{\text {med }}^{\prime}}{\tilde{\varepsilon}_{c e l l}^{\prime}+2 \tilde{\varepsilon}_{\text {med }}^{\prime}}, \\
& \tilde{\varepsilon}_{\text {cell }}^{\prime}=\tilde{\varepsilon}_{\text {mem }}\left[\frac{\left(\frac{R_{c y t o}}{R_{\text {mem }}}\right)^{3}+2\left(\frac{\tilde{\varepsilon}_{c y t o}-\tilde{\varepsilon}_{\text {mem }}}{\tilde{\varepsilon}_{c y t o}+2 \tilde{\varepsilon}_{\text {mem }}}\right)}{\left(\frac{R_{c y t o}}{R_{\text {mem }}}\right)^{3}-\left(\frac{\tilde{\varepsilon}_{c y t o}-\tilde{\varepsilon}_{\text {mem }}}{\tilde{\varepsilon}_{c y t o}+2 \tilde{\varepsilon}_{m e m}}\right)}\right],
\end{aligned}
$$




$$
\tilde{\varepsilon}_{i}=\varepsilon_{i}+\frac{\sigma_{i}}{\omega j}
$$

where $\mathrm{R}$ is the radius of the cell cytoplasm and membrane ( $\mathrm{R}_{\text {cyto }}$ and $\left.\mathrm{R}_{\text {mem }}\right), \tilde{\boldsymbol{\varepsilon}}_{\mathbf{i}}$ is the complex permittivity, $\varepsilon_{\mathrm{i}}$ is the permittivity, and $\sigma_{\mathrm{i}}$ is the conductivity where $i=$ cell, cytoplasm, membrane, or medium. The complex permittivity $\tilde{\boldsymbol{\varepsilon}}_{\boldsymbol{i}}$, is also dependent on the angular frequency $(\boldsymbol{\omega})$ [41]. $f_{C M}$ is dependent on the complex permittivity of the cell and the medium, equation 2 . If a cell experiences pDEP then $f_{C M}$ (equation 1 ) is positive indicating the cell is more polarizable than the suspending medium, $\tilde{\varepsilon}_{\text {cell }}^{\prime}>$ $\widetilde{\varepsilon}_{m e d}$, and the cell moves toward areas of high electric field density [41]. For nDEP, the $f_{C M}$ is negative and the cell is less polarizable than the suspending medium, $\widetilde{\varepsilon}_{\text {med }}>$ $\tilde{\varepsilon}_{c e l l}^{\prime}$, and the cell moves towards areas of low electric field density [41]. When $f_{C M}$ is zero, known as the cross-over frequency $f_{x o}[39]$, cells experience no DEP force $\left(F_{D E P}\right.$ $=0$ ) as they transition from $\mathrm{nDEP}$ to $\mathrm{pDEP}$ or $\mathrm{pDEP}$ to $\mathrm{nDEP}$. This $f_{x o}$ is an important component of a cell's DEP spectra because the dielectric properties of cells can be estimated along with other data points from the DEP spectra.

The DEP behavior of cells is quantified experimentally by measuring the $\mathrm{pDEP}$ and $\mathrm{nDEP}$ at specific frequencies within a given buffer solution. The $f_{x o}$ can be extrapolated between the two nearest $\mathrm{pDEP}$ and $\mathrm{nDEP}$ frequencies. Cells typically display two $f_{x o}$ 's, which are dominated by its membrane structure. The low $f_{x o}$ is determined by the cell's size, shape, and membrane with typical values between 0.010 $0.10 \mathrm{MHz}$ ( $\beta$-region), but reported as high as $\sim 4 \mathrm{MHz}[43]$. The high $f_{x o}$ is dominated 
by the cell's cytoplasm and is typically above 10MHz in low conductivity media [44]. For the experiments described in this work, the high $f_{x o}$ value falls outside of the range of our function generator ( $\gamma$-region). Membrane capacitance, $C_{m e m}$, is a function of $f_{x o}$ and given by $[44,45]$

$$
C_{m e m}=\frac{\sqrt{2} \sigma_{m e d}}{2 \pi r f_{x o}},
$$

where $\mathrm{r}$ is hMSCs radius $(\mu \mathrm{m})$. Further, the membrane permittivity is proportional to $C_{m e m}$,

$$
\varepsilon_{m e m}=\frac{C_{m e m} d}{4 \pi r^{2} \varepsilon_{0}}
$$

where $d$ is the membrane thickness and $\varepsilon_{0}$ is the vacuum permittivity. Therefore, the $f_{x o}$ and $C_{m e m}$ can be used as indicators of treatment-induced hMSC changes and can be lumped into $\varepsilon_{m e m}$, a key dielectric property.

To eliminate variation in cell morphology and increase DEP response reproduciblity within our hMSC sample population, elastin-like polypeptide (ELP) polyethyleneimine (PEI) was employed. ELP-PEI is a postively charged copolymer that directs cells morphology to a spheroidal shape with a propensity to form aggregates. Similar to mammalian elastin, ELP is synthesized from amino acids including valine, proline, and glycine and has been utilized to induce spheroid formation and differentiation of $\mathrm{H} 35$ rat hepatocytes. ELP-PEI has also elucitated affects of free fatty acids and cytokines in 2D and 3D rat hepatoma cell cultures [46, 47]. 
In this paper, the first steps toward the development of a continuous cell sorting microdevice were completed by characterizing the dielectric signature of hMSCs and standardizing their morphology with ELP-PEI treatments. We demonstrate that DEP can quantify hMSCs $\varepsilon_{\mathrm{mem}}, \sigma_{\mathrm{mem}}$, and $\mathrm{C}_{\mathrm{mem}}$ based on measured DEP spectra. We also characterize the DEP behavior of ELP-PEI treated hMSCs for comparison to the native hMSCs. Determing hMSCs dieletric properties and removing morphology variations is essential to increase reproducibility and magnify different membrane biosurface markers.

\subsection{Materials and Methods}

Microdevice Fabrication: The microdevice in Figure 4.1b was fabricated using prior published techniques [30] with $100 \mu \mathrm{m}$ wide and $200 \mu \mathrm{m}$ spaced electrodes. A $2000 \mu \mathrm{m}$ deep by $3000 \mu \mathrm{m}$ wide microwell made with PDMS was sealed on top of the quadrapole array. Figure $4.1 \mathrm{~b}$ shows the quadrapole Ti-Au electrode microdevice setup.

hMSC Cell Culture: Nitrogen stored bone marrow derived hMSCs were recovered by thawing cells in $37^{\circ} \mathrm{C}$ water bath. $500 \mathrm{~mL}$ alpha minimum essential medium ( $\alpha \mathrm{MEM}$ ) with L-glutamine, without ribonucleosides or deoxyribonucleosides (Catalog \# M20350, Atlanta Biologicals, Atlanta, GA) was completed with 50mL fetal bovine serum (FBS, Catalog \# S11550, Atlanta Biologicals), 3mL L-glutamine (200mM in $0.85 \% \mathrm{NaCl}$, Catalog \# 25030081, Life Technologies, Carlsbad, CA), and 3mL penicillin/streptomycin (Catalog \# 15140122, Life Technologies). Thawed 
hMSCs were placed in $15 \mathrm{~mL}$ complete $\alpha \mathrm{MEM}$ in sterile cell culture flask and incubated overnight at $37^{\circ} \mathrm{C}, 5 \% \mathrm{CO}_{2}$. After $24 \mathrm{hrs}$, phosphate buffer solution (PBS, Catalog \# 6508-OP, EMD Millipore, Chicago, IL), 0.25\% trypsin/1mM EDTA (Catalog \# 25200072, Life Technologies) and complete $\alpha \mathrm{MEM}$ were warmed to $37^{\circ} \mathrm{C}$. Old complete aMEM was removed from the flask and attached hMSCs were washed with $10 \mathrm{ml}$ PBS. $4 \mathrm{~mL}$ trypsin was added to the flask and hMSCs were incubated at $37^{\circ} \mathrm{C}, 5 \% \mathrm{CO}_{2}$ for $3-4$ mins for full cell detachment (monitored with microscope). After detachment $5 \mathrm{~mL}$ of complete $\alpha \mathrm{MEM}$ was added to flask to inactivate trypsin, and the hMSC suspension was placed in sterile centrifuge tubes. hMSCs were centrifuged for 10 minutes at 494 relative centrifugal force (RCF). The supernatant was removed and hMSCs were resuspended in $5 \mathrm{~mL}$ of fresh completed aMEM. $1 \mathrm{~mL}$ of hMSC suspension was placed in new cell culture flask with $15 \mathrm{~mL}$ complete $\alpha \mathrm{MEM}$ for the next passage. hMSCs were incubated until 90\% confluent ( $\sim 5$ days) and passaged following the same trypsin detachment procedure. Multiple flasks of hMSCs were cultured for DEP tests, with an extra hMSC flask continually passaged every 4-5days for subsequent DEP testing. DEP tests were completed when cells reached $10^{6}$ cell $/ \mathrm{mL}$ concentration ( $\sim 5-7$ days).

DEP Solution Preparation: 1M NaOH (Catalog \#S318-500, Fisher Scientific, Waltham, MA) solution, 3M salt stock solution, 4\% bovine serum albumin (BSA, Catalog \# A7906-100G, Sigma Aldrich, St. Louis, MO)-Epure $\mathrm{H}_{2} \mathrm{O}$ pH 10 solution, $0.030 \mathrm{~S} / \mathrm{m}$ and $0.10 \mathrm{~S} / \mathrm{m}$ dextrose solutions were prepared to complete hMSCs DEP

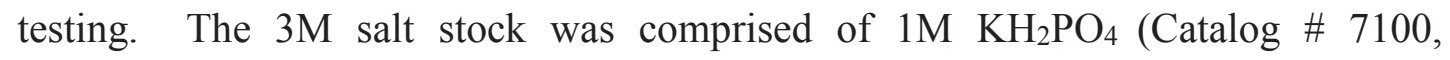


Mallinckrodt Chemicals, St. Louis, MO), $1 \mathrm{M} \mathrm{NaCl}$ (Catalog \# 7581, Macron Chemicals, Swedesboro, NJ), and 1M K2 $\mathrm{HPO}_{4}$ (Catalog \# BDH0266-500G, VWR, West Chester, PA) in $20 \mathrm{~mL}$ Epure $\mathrm{H}_{2} \mathrm{O}$. A 4\% BSA pH 10 solution was utilized to pretreat the microdevice surface. The $0.030 \mathrm{~S} / \mathrm{m}$ dextrose solution was prepared to $0.30 \mathrm{M}$ with $0.30 \mathrm{M}$ dextrose (Catalog \# D9434-500G, Sigma Aldrich) and 9.1 $\times 10^{-4} \mathrm{M}$ salt stock, then balanced to $\mathrm{pH} 7$ with $1 \mathrm{M} \mathrm{NaOH}$ (as necessary). 4\% BSA was added and thoroughly vortexed. The $0.10 \mathrm{~S} / \mathrm{m}$ dextrose solution was prepared to $0.28 \mathrm{M}$ with $0.27 \mathrm{M}$ dextrose and $7.5 \times 10^{-3} \mathrm{M}$ salt stock, then balanced to $\mathrm{pH} 7$ with $1 \mathrm{M} \mathrm{NaOH}$ (as necessary). 4\% BSA was added and thoroughly vortexed. Final dextrose solution conductivities were verified using a conductivity meter (AB30, Fisher Scientific). 
(a)
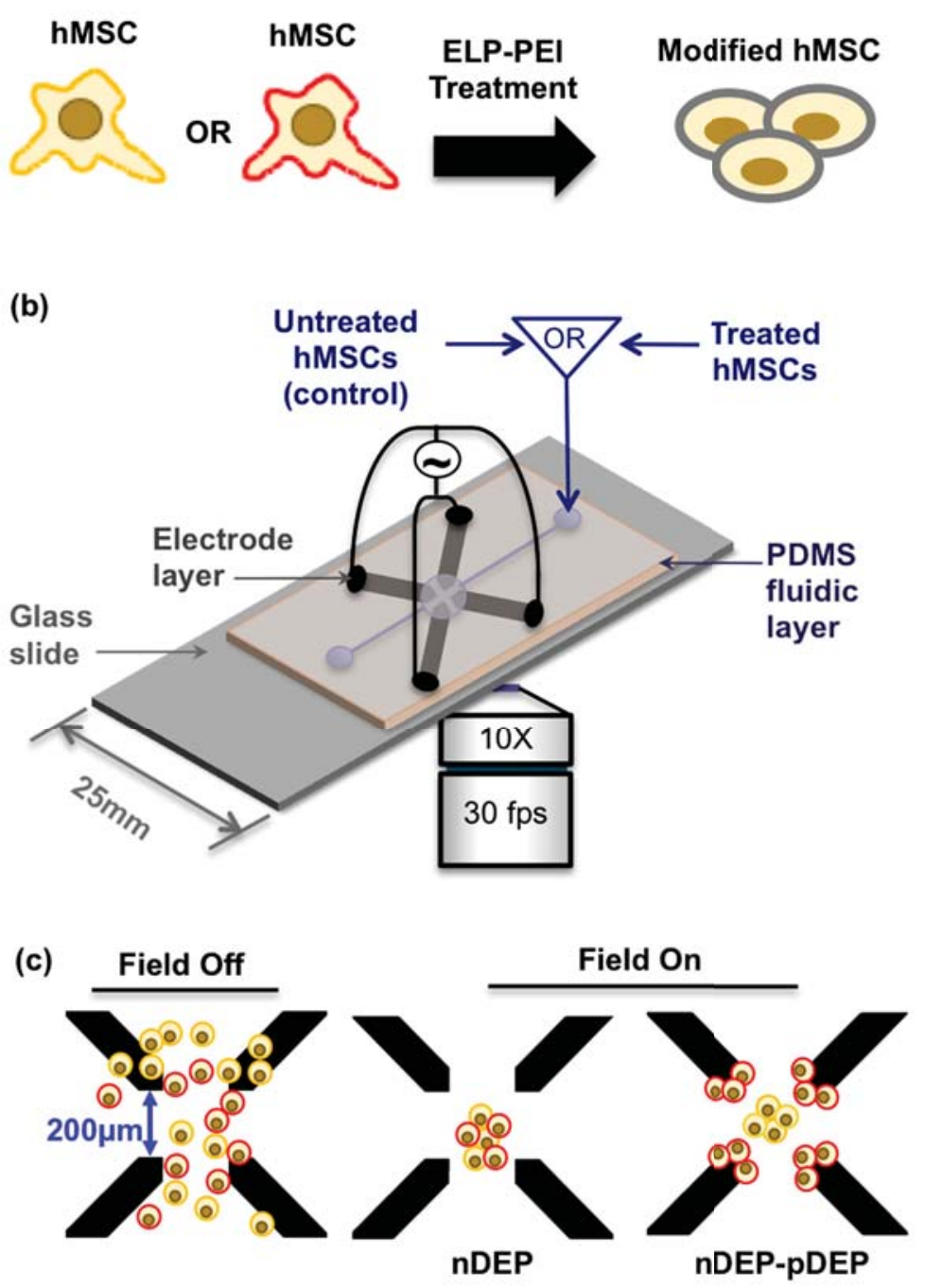

Figure 4.1 (a) hMSCs cartooned to emphasize the differing cell membrane biosurface markers and thus properties. After ELP-PEI treatment, cartooned spheroidal morphology. (b) Quadrapole microdevice with $200 \mu \mathrm{m}$ spaced Ti-Au electrodes used for DEP experiments. (c) hMSCs in device with field off, and the DEP response observed with field on. At a single frequency, both $\mathrm{nDEP}$ and $\mathrm{pDEP}$ were observed due to differing membrane biosurface markers within an hMSCs population. 
DEP Experimental Procedure: For DEP tests, centrifuged untreated hMSCs were resuspended to a concentration of $1.3 \times 10^{6}$ cells $/ \mathrm{mL}$ in either $0.030 \mathrm{~S} / \mathrm{m}$ or $0.10 \mathrm{~S} / \mathrm{m}$ dextrose solution. The microdevice was presoaked in 4\% BSA-Epure $\mathrm{H}_{2} \mathrm{O}$ adjusted to $\mathrm{pH} 10$ to prevent cell adhesion. The hMSC dextrose suspension was loaded into the quadrapole chamber using a micropipette. A function generator (Agilent 33250A, Agilent, Santa Clara, CA) was connected to the microdevice via copper leads (Figure 4.1b). Static frequency measurements were completed using 10Vpp AC sine wave, $0.010 \mathrm{MHz}$ to $35 \mathrm{MHz}$ for $90 \mathrm{~s}$. More than 300 untreated hMSCs $(\mathrm{n}>300)$ were tested at each static frequency. Untreated hMSCs static DEP responses were compared to static measurements of ELP-PEI treated hMSCs. To achieve ELP-PEI hMSC treatment, ELP-PEI was synthesized as described in $[46,47]$ and dissolved in Epure $\mathrm{H}_{2} \mathrm{O}(5 \mathrm{mg} / \mathrm{mL})$ in a sterile centrifuge tube. $5 \mathrm{~mL}$ ELP-PEI Epure $\mathrm{H}_{2} \mathrm{O}$ solution was added to a vented cell culture flask and dried for $48 \mathrm{hrs}$ at $37^{\circ} \mathrm{C}$. After $48 \mathrm{hrs}$, excess water was removed and the coated flask was decontaminated under UV light for 5mins. $90 \%$ confluent hMSCs were trypsinized and placed in ELP-PEI coated flask for 24hrs. hMSC images were taken before and after ELP-PEI treatment. After 24 hours, ELP-PEI treated hMSCs were tested within the DEP microdevice using AC fields and static frequencies.

Data Acquisition: 30 fps video recordings using LabSmith SVM Synchronized Video Microscope 10x objective (LabSmith, Livermore, CA, USA) were collected. DEP behaviors of the untreated hMSCs and ELP-PEI treated hMSCs were quantified 
with ImageJ (NIH, Bethesda, MD) by recording cell position and number after 90s in the AC electric field. Percent cell responses $\left(\mathrm{R}_{\mathrm{c}}\right)$ were calculated by tabulating the number of cells, $\mathrm{n}_{\mathrm{i}}$, exhibiting $\mathrm{nDEP}$ and $\mathrm{pDEP}$ behaviors (where $i=\mathrm{nDEP}, \mathrm{pDEP}$, pDEP-nDEP) divided by the total number of cells, $\mathrm{n}_{\mathrm{T}}$, tested.

$$
R_{c}(\%)=\frac{n_{i}}{n_{T}} \times 100,
$$

Rc was plotted as a stacked column chart to show hMSC nDEP, pDEP, and pDEP-nDEP behavior at individual static frequencies, Figure 4.3a and 4.3b. Next, Rc was translated to a traditional DEP spectra plot (inspired by [48]) by scaling the nDEP responses by $f_{C M, \min }$ and $\mathrm{pDEP}$ responses by $f_{C M, \max }$ calculated via Equations 1-3 for each conductivity. The scaled equations were,

$$
\begin{aligned}
& R_{c, n D E P}=\frac{n_{n D E P}}{n_{T}} \times f_{C M \text { min }}, \\
& R_{c, p D E P}=\frac{n_{P D E P}}{n_{T}} \times f_{C M \text { max }} .
\end{aligned}
$$

At $0.030 \mathrm{~S} / \mathrm{m}, f_{C M, \min }=-0.45$ and $f_{C M, \max }=0.72$ and for $0.10 \mathrm{~S} / \mathrm{m}, f_{C M, \min }=-0.49$ and $f_{C M, \max }=0.36$. If the magnitude of the scaled $R_{c, n D E P}$ was larger than the scaled $\mathrm{R}_{c, \mathrm{pDEP}}$ then $R_{c, n D E P}$ was selected if not then $R_{c, p D E P}$ was selected. The DEP spectra transitions from nDEP to pDEP when $R_{c, n D E P} \cong R_{c, p D E P}=0.50$ (at the $f_{x o}$ ) as illustrated in Figure $4.3 \mathrm{c}$.

Comparison to Model: The resulting DEP spectra for untreated and ELP-PEI treated hMSCs were separately fit to the core-shell spherical DEP polarization model. The starting literature values were $\varepsilon_{\mathrm{mem}}=6.5[49-51,14,52], \sigma_{\mathrm{mem}}=10^{-6}[49-51,14]$, 
$\varepsilon_{\text {cyto }}=60[49,50,14,52], \sigma_{\text {cyto }}=0.63 \mathrm{~S} / \mathrm{m}[49-51,14,52]$. These dielectric parameters ( $\varepsilon_{\text {mem }}, \sigma_{\text {mem }}, \varepsilon_{\text {cyto }}$, and $\sigma_{\text {cyto }}$ ) were iteratively adjusted in Equations 1-3 starting from the literature values, then varied to obtain the best curve fit to experimental data. $\mathrm{R}=$ $20 \mu \mathrm{m}$ was used for each optimization. $\mathrm{C}_{\mathrm{mem}}$ was calculated from $f_{x o}$ to obtain parameters for $\varepsilon_{\text {mem }}$ via equations 4 and 5 .

\subsection{Results and Discussion}

Static frequency measurements were completed from $0.010 \mathrm{MHz}$ to $35 \mathrm{MHz}$ to establish hMSCs DEP response spectra to then calculate untreated and ELP-PEI treated hMSCs dielectric properties. Static measurements were completed at 10Vpp for $90 \mathrm{~s}$ at each individual frequency and Figure 4.2 illustrates the hMSC spatial cell counts and subsequent $R_{c}$ at $0.010,1.0$, and $10 \mathrm{MHz}$ for untreated hMSCs. The notation nDEP-pDEP indicates cells that initially exhibit pDEP and then transition to nDEP, a behavior that is pronounced near $f_{x o}$ (see Figure 4.3). 
(a) Untreated hMSCs

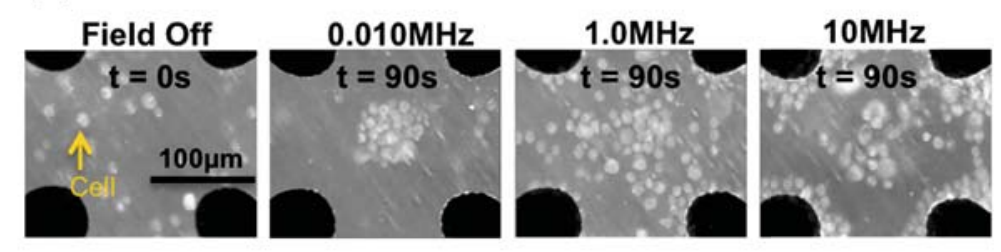

(b)

\begin{tabular}{|c|c|c|c|}
\hline Total \# cells & 37 & 98 & 112 \\
\hline \# nDEP & 37 & 61 & 23 \\
\hline \# pDEP & 0 & 37 & 89 \\
\hline
\end{tabular}

(c)

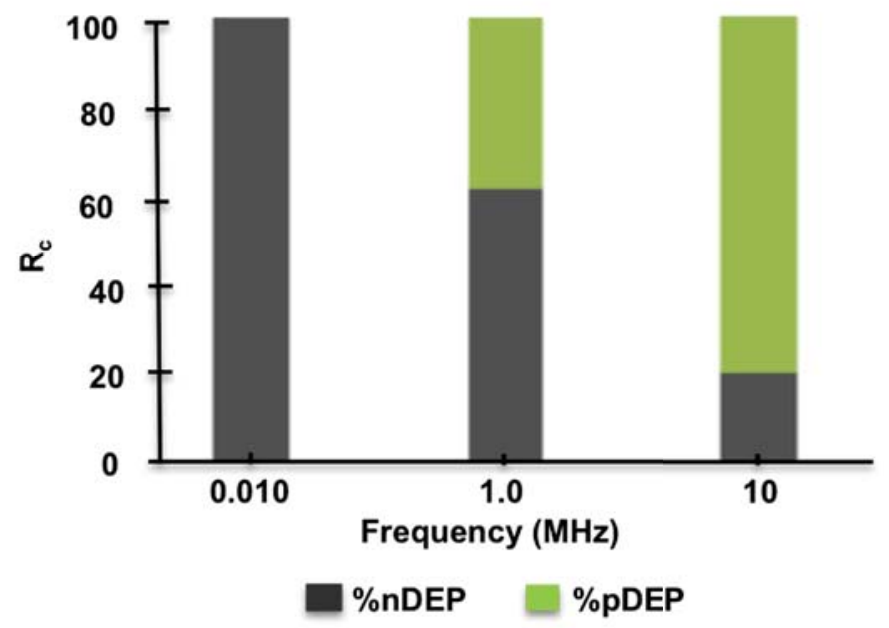

Figure 4.2 (a) Microscope images of untreated hMSCs DEP response with the field off and at $0.010,1.0$, and $10 \mathrm{MHz}$ after $90 \mathrm{~s}$ in $0.10 \mathrm{~S} / \mathrm{m}$ dextrose solution. At 0.010MHz, hMSCs only display nDEP and display both nDEP and pDEP at 10MHz. (b) Table of total cells for each image. (c) DEP responses tabulated as $\%$ cell response $\left(\mathrm{R}_{\mathrm{c}}\right)$ into a stacked column chart. nDEP dominates at $0.010 \mathrm{MHz}$ and at $10 \mathrm{MHz} \mathrm{pDEP}$ is $79 \%$ dominant.

To examine the medium conductivity dependence, hMSCs were tested in $0.030 \mathrm{~S} / \mathrm{m}$ and $0.10 \mathrm{~S} / \mathrm{m}$ at fixed $10 \mathrm{Vpp}$ for $90 \mathrm{~s}$ at each individual frequency. 
Experiments were rerun until $n>300$ cells and were tabulated at each frequency. Figure $4.3 \mathrm{a}$ and $4.3 \mathrm{~b}$ show the stacked column representation of hMSCs DEP response in $0.030 \mathrm{~S} / \mathrm{m}$ and $0.10 \mathrm{~S} / \mathrm{m}$, respectively. In $0.030 \mathrm{~S} / \mathrm{m}$ media, $\mathrm{nDEP}$ (gray) is the dominant behavior up to $\sim 0.65 \mathrm{MHz}$, at which point the DEP behavior transitions to $\mathrm{pDEP}$ (green) dominance. Individual cells experiencing both $\mathrm{pDEP}$ and $\mathrm{nDEP}$ (red) within one experimental run are seen between $0.10-2 \mathrm{MHz}$. In $0.10 \mathrm{~S} / \mathrm{m}$ media, nDEP is dominant until $\sim 1 \mathrm{MHz}$ and then transitions to pDEP behavior following a similar trend as the lower conductivity but with the frequency response shifted higher. Figure $4.3 \mathrm{c}$ illustrates the translation of the $R_{c}$ responses to hMSCs DEP spectra at $0.030 \mathrm{~S} / \mathrm{m}$ and $0.10 \mathrm{~S} / \mathrm{m}$, respectively. The DEP spectra curve shift is more apparent with this plot format. Thus, hMSCs display different $f_{x o}$ 's dependent on the conductivity of the suspending solution, $0.030 \mathrm{~S} / \mathrm{m} f_{x o}=0.62 \mathrm{MHz}$ and $0.10 \mathrm{~S} / \mathrm{m} f_{x o}=1.3 \mathrm{MHz}$. The frequency shift of $0.68 \mathrm{MHz}$ is consistent with other medium conductivity experiments in the literature [39]. It was also observed that the DEP force weakened (slower hMSC movement) as the $f_{x o}$ was approached. 
(a)

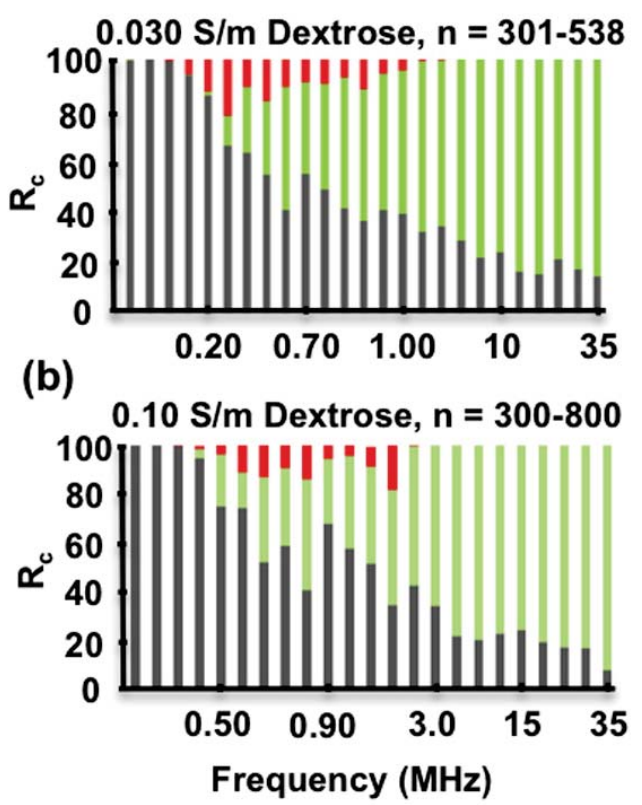

(c)

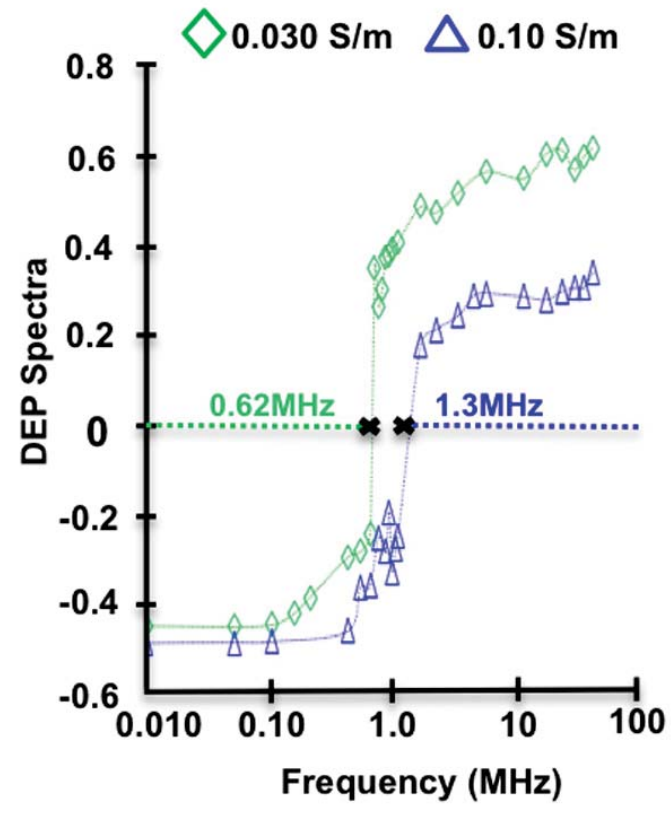

\%nDEP \%pDEP — \%pDEP-nDEP

Figure 4.3 (a) Percent cell response $R_{c}$ for untreated hMSCs in $0.030 \mathrm{~S} / \mathrm{m}$ dextrose solution. (b) $R_{c}$ for untreated hMSCs in $0.10 \mathrm{~S} / \mathrm{m}$ dextrose solution. For both conductivities, $\mathrm{nDEP}$ dominates lower frequencies while $\mathrm{pDEP}$ dominates higher frequencies. (c) Data from a and b translated into DEP response spectra for untreated hMSCs at $0.030 \mathrm{~S} / \mathrm{m}$ and $0.10 \mathrm{~S} / \mathrm{m}$. This format best illustrates $0.030 \mathrm{~S} / \mathrm{m} f_{x o}$ estimated as $0.62 \mathrm{MHz}$ and $0.10 \mathrm{~S} / \mathrm{m} f_{x o}$ estimated as $1.3 \mathrm{MHz}$.

The experimental data in Figure 4.3 was compared to the core-shell spherical DEP polarization model (equations 1 - 3) as illustrated in Figure 4.4. The experimental $f_{x o}$ 's were used in equations 4 and 5 to calculate $\mathrm{C}_{\mathrm{mem}}$ and $\varepsilon_{\mathrm{mem}}$ of $2.2 \mathrm{pF}$ and $2.0,4.5 \mathrm{pF}$ and 4.1 for $0.030 \mathrm{~S} / \mathrm{m}$ and $0.10 \mathrm{~S} / \mathrm{m}$, respectively. The $\varepsilon_{\mathrm{mem}}$ 's were utilized along with starting model parameters specified in materials and methods and optimized to $\varepsilon_{\mathrm{mem}}=$ 
$0.79, \sigma_{\text {mem }}=10^{-6}, \varepsilon_{\text {cyto }}=60$, and $\sigma_{\text {cyto }}=0.50 \mathrm{~S} / \mathrm{m}$ for $0.030 \mathrm{~S} / \mathrm{m}$ (Figure $4.4 \mathrm{a}$ solid curve and Table 4.1). $0.030 \mathrm{~S} / \mathrm{m}$ experimental data (open green diamonds) has good agreement with the core-shell spherical polarization model, with an overall $23 \%$ error. For $0.10 \mathrm{~S} / \mathrm{m}$, model parameters were separately optimized to $\varepsilon_{\mathrm{mem}}=1.1, \sigma_{\mathrm{mem}}=10^{-6}$, $\varepsilon_{\text {cyto }}=60, \sigma_{\text {cyto }}=0.50 \mathrm{~S} / \mathrm{m}$ (Figure $4.4 \mathrm{a}$ dashed curve and Table 4.1$)$ and agreed well with the experimental data (open blue triangles), with an overall $17 \%$ error.

(a)

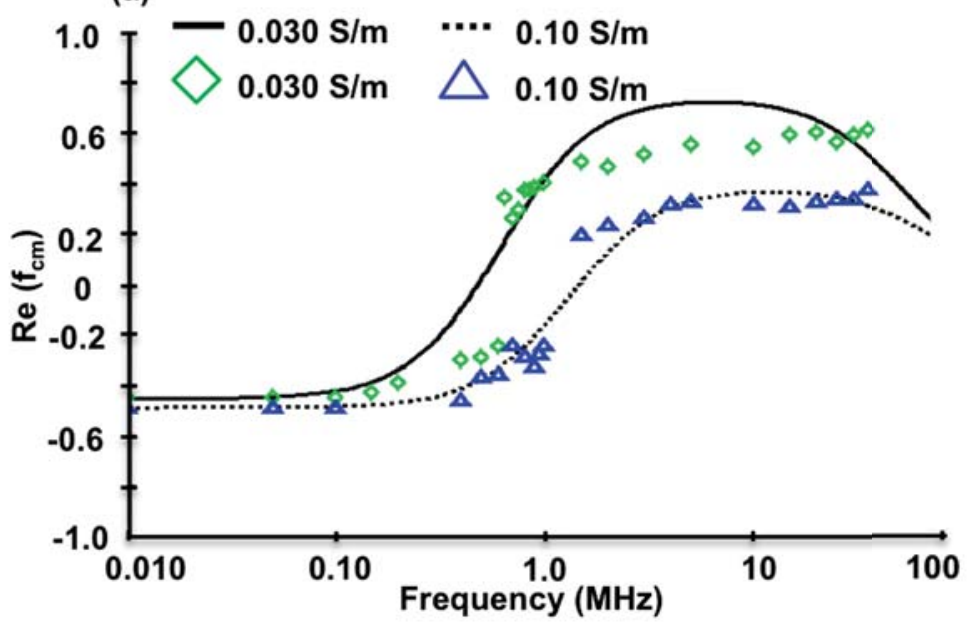

Experimental $\mid$ Wheoretical

(b)

(c)
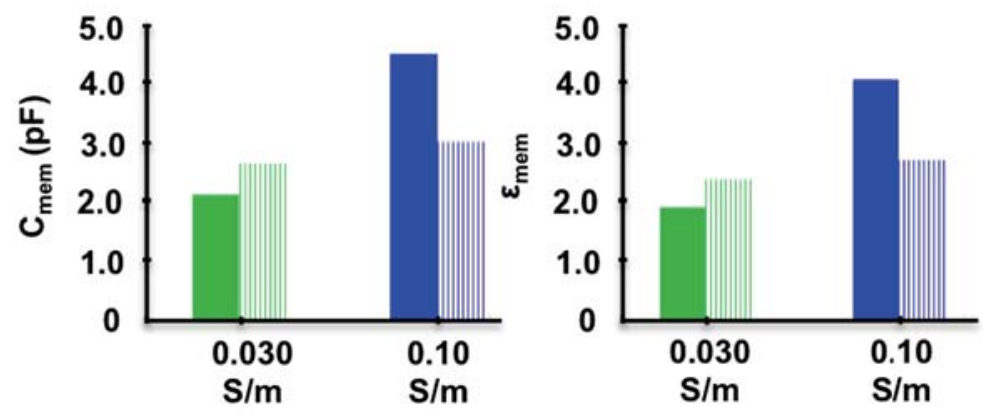

Figure 4.4 (a) Untreated hMSC DEP responses at 0.030 and $0.10 \mathrm{~S} / \mathrm{m}$ compared to the core-shell spherical model. (b) hMSC membrane capacitance and (c) membrane permittivity based on experimentally determined cross-over frequency. 
In order to elucidate the morphology contribution to the DEP response spectra, ELP-PEI treated hMSCs were examined following the same experimental procedures in $0.10 \mathrm{~S} / \mathrm{m}$. Figure 4.5 compares ELP-PEI treated to untreated hMSCs experimental images to DEP response spectra. Figures $4.5 \mathrm{a}$ and $4.5 \mathrm{~b}$ illustrate the morphological changes between untreated hMSCs attached to the bottom of the cell culture flask and ELP-PEI treated hMSCs spheroidal cells and cell aggregates, which do not adhere to the flask bottom. These images indicate that treated hMSCs formed spheroidal aggregates consistent with liver cells, H35 rat hepatoma cells, and 3T3 mouse cells described in previous literature $[46,47]$; therefore the ELP-PEI treatment on the hMSCs was successful in producing spheroidal morphology. Figures $4.5 \mathrm{c}$ and $4.5 \mathrm{~d}$ compare untreated and ELP-PEI treated hMSCs within the DEP microdevice with the electric field off. With the field off, hMSC suspensions were randomly distributed within the microwell, although cell aggregates were apparent with the treated hMSCs. Figures $4.5 \mathrm{e}$ and $4.5 \mathrm{f}$ compare untreated and ELP-PEI treated hMSCs at $0.010 \mathrm{MHz}$ after $90 \mathrm{~s}$ in the AC field. Both the untreated and treated hMSCs display nDEP behavior, but the treated hMSCs were more aggregated. At increased frequencies 1.0MHz (Figure 4.5g and 4.5h) and 10MHz (Figure 4.5i and 4.5j), untreated hMSCs exhibit both nDEP and pDEP behavior while ELP-PEI treated hMSCs only exhibit nDEP.

Figure 4.5k the corresponding DEP spectra for untreated hMSCs (open red triangles) and treated hMSCs (open red triangles). At high frequencies $(>10 \mathrm{MHz})$, ELP-PEI treated hMSCs exhibit primarily nDEP with minor pDEP behaviors. 
However, untreated hMSCs reveal nDEP behavior at lower frequencies, and pDEP at higher frequencies with the $f_{x o} \sim 1.3 \mathrm{MHz}$. There was not a $f_{x o}$ value within the tested frequency range for treated hMSCs, so $\mathrm{C}_{\mathrm{mem}}$ and $\varepsilon_{\mathrm{mem}}$ were estimated to be $>0.13 \mathrm{pF}$ and $>0.12$ with $\mathrm{f}_{\mathrm{xo}}>35 \mathrm{MHz}$. For untreated hMSCs, cell counts were at least 300 for each static frequency tested. Treated hMSCs were aggregated spheroids, therefore the cell counts are denoted n'. Lower n' numbers were tolerated at lower frequencies where nDEP clearly dominated the responses.

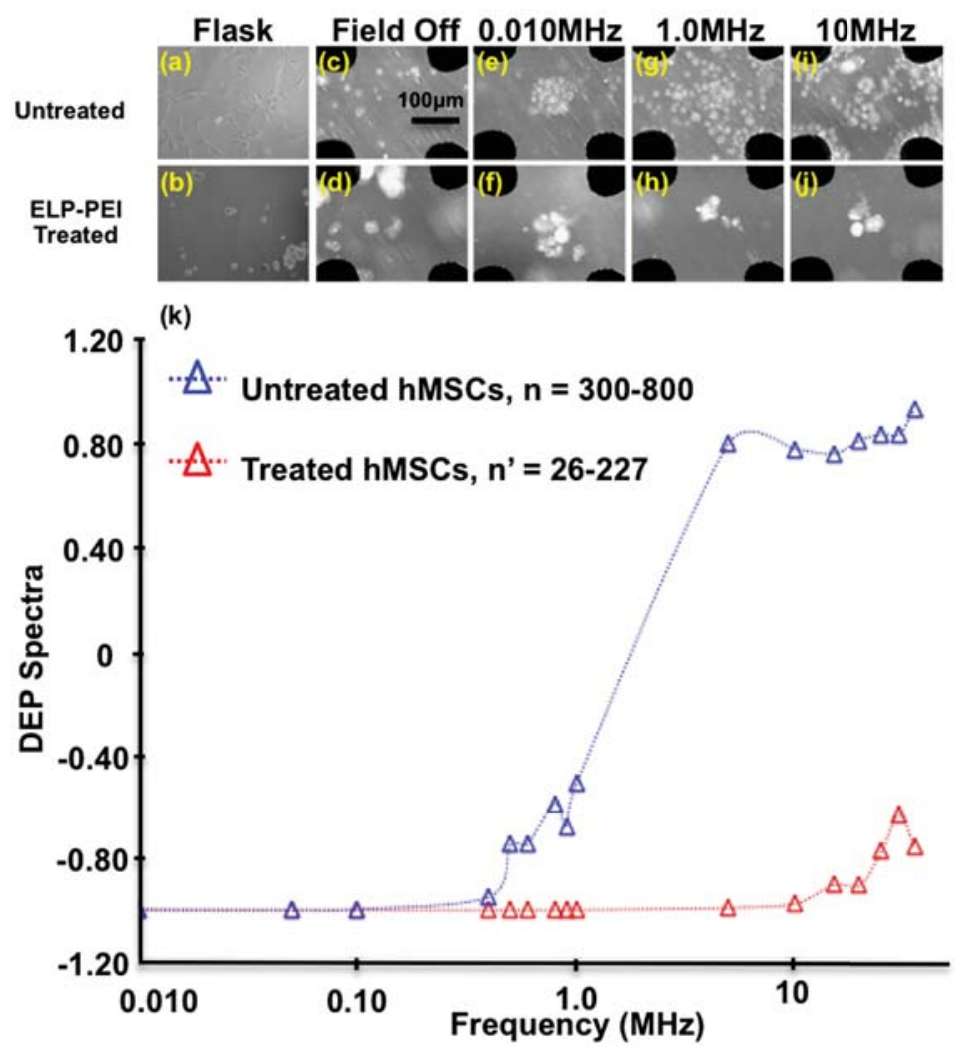

Figure 4.5 Untreated hMSCs (first row) compared to ELP-PEI treated hMSC spheroidal morphology (second row), both in $0.10 \mathrm{~S} / \mathrm{m}$ dextrose solution. (a) untreated hMSCs in cell culture flask, (b) treated hMSCs after 24hrs in cell culture flask, (c) 
untreated and (d) treated hMSCs in microdevice with field off where black regions are the quadrapole electrodes. Untreated and treated hMSCs at $10 \mathrm{Vpp}$ and $0.010 \mathrm{MHz}$ (e and f), $1.0 \mathrm{MHz}$ (g and h), and 10MHz (i and j). (k) DEP response spectra of untreated and ELP-PEI treated hMSCs in $0.10 \mathrm{~S} / \mathrm{m}$. Untreated cells transition from $\mathrm{nDEP}$ to pDEP at $\sim 1.3 \mathrm{MHz}$, while treated cells predominantly exhibit nDEP behaviors and formed spheroidal aggregates.

The ELP-PEI treated hMSCs DEP experimental data at $0.10 \mathrm{~S} / \mathrm{m}$ was also fit to the core-shell spherical DEP polarization model as shown in Figure 4.6. Because $f_{x o}$ could not be directly measured, model parameters $\varepsilon_{\mathrm{mem}}, \sigma_{\mathrm{mem}}, \varepsilon_{\mathrm{cyto}}$, and $\sigma_{\text {cyto }}$ were systematically adjusted from theoretical values to arrive at best fit parameters in Figures 4.6a through 4.6d, respectively. For each individual parameter optimization, the other three literature values were held constant as illustrated in Table 4.1. Membrane permittivity adjustments in Figure 6a revealed that decreasing $\varepsilon_{\mathrm{mem}}$ from 5 $[49-51,14,52]$ to 0.050 better matched the experimental data. Membrane conductivity adjustments in Figure $4.6 \mathrm{~b}$ from $\sigma_{\mathrm{mem}} 10^{-5}$ down to $10^{-7}[49-51,14]$ had very little impact on achieving model/data agreement. Cytoplasm permittivity adjustments in Figure $4.6 \mathrm{c}$ from $\varepsilon_{\mathrm{mem}} 6$ up to $100[49,50,14,52]$ also had very little impact on achieving model/data agreement. Cytoplasm conductivity adjustments in Figure 4.6d from $\sigma_{\text {cyto }} 0.63 \mathrm{~S} / \mathrm{m}[49-51,14,52]$ down to $6.3 \times 10^{-3} \mathrm{~S} / \mathrm{m}$, achieved good agreement with experimental data and may account for the nDEP behavior displayed by the ELPPEI treated hMSCs. Figure 4.6e compares the optimized model parameters for untreated and treated hMSC. These values are simultaneously summarized in Table 
4.1. All final model parameters lie within the range of previously reported literature values, except the membrane permittivity and cytoplasm conductivity, which were slightly lower than the lowest reported value of $6.5[49-51,14,52]$ and $0.63 \mathrm{~S} / \mathrm{m}[49-$ $51,14,52]$, respectively.
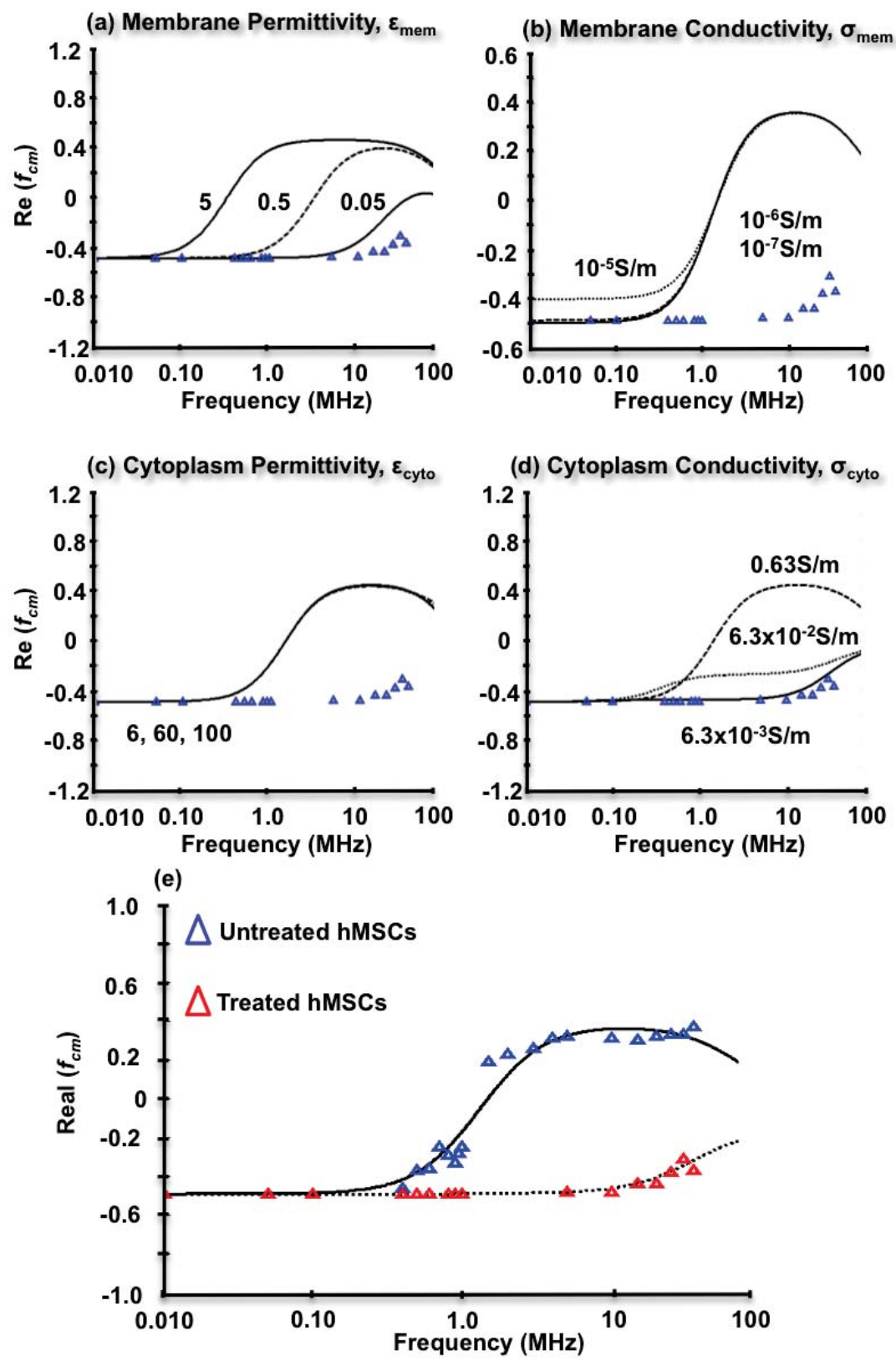
Figure 4.6 Key parameter optimizations for the core-shell spherical DEP polarization model for ELP-PEI treated hMSCs at $0.10 \mathrm{~S} / \mathrm{m}$. (a) Decreases from literature values in membrane permittivity better capture ELP-PEI treated hMSCs nDEP behaviors. (b) Increases or decreases in membrane conductivity do not contribute to the experimentally observed nDEP. (c) Decreases in cytoplasm permittivity also do not contribute. (d) Decreases in cytoplasm conductivity may contribute to the nDEP behavior with $\sigma_{\text {cyto }}=6.3 \times 10^{-3} \mathrm{~S} / \mathrm{m}$ fitting well. (e) Model prediction for both untreated and treated hMSCs at $0.10 \mathrm{~S} / \mathrm{m}$ using all optimal parameters for each. Agreement between the model and the data is quite good with an overall $2.5 \%$ error.

Table 4.1 Summary of literature and optimized parameters for core-shell spherical DEP polarization model for untreated and treated hMSCs in $0.030 \mathrm{~S} / \mathrm{m}$ and $0.10 \mathrm{~S} / \mathrm{m}$.

\begin{tabular}{|c|c|c|c|c|}
\hline & Emem & $\begin{array}{c}\sigma_{\text {mem }} \\
(\mathbf{S} / \mathbf{m})\end{array}$ & Ecyto & $\sigma_{\text {cyto }}(\mathrm{S} / \mathrm{m})$ \\
\hline $\begin{array}{r}\text { Literature Range } \\
\text { (starting model } \\
\text { values) } \\
\text { [references] }\end{array}$ & $\begin{array}{r}6.5-11 \\
(0.50) \\
{[49-51,} \\
14,52] \\
\end{array}$ & $\begin{array}{c}10^{-3}-10^{-8} \\
\left(10^{-6}\right) \\
{[49-51} \\
14]\end{array}$ & $\begin{array}{c}50-100 \\
(60) \\
{[49,50,} \\
14,52]\end{array}$ & $\begin{array}{c}0.30-0.88 \\
(0.63) \\
{[49-51} \\
14,52] \\
\end{array}$ \\
\hline $\begin{array}{r}\text { Untreated } \\
\mathbf{0 . 0 3 0 S} / \mathrm{m} \\
\end{array}$ & 0.79 & $10^{-6}$ & 60 & 0.50 \\
\hline $\begin{array}{r}\text { Untreated } \\
0.10 \mathrm{~S} / \mathrm{m}\end{array}$ & 1.1 & $10^{-6}$ & 60 & 0.50 \\
\hline $\begin{array}{r}\text { ELP-PEI Treated } \\
0.10 \mathrm{~S} / \mathrm{m}\end{array}$ & 0.050 & $10^{-6}$ & 60 & $6.3 \times 10^{-3}$ \\
\hline
\end{tabular}

In review, there was an observable conductivity dependence on the DEP behavior of untreated hMSCs in $0.030 \mathrm{~S} / \mathrm{m}$ and $0.10 \mathrm{~S} / \mathrm{m}$. This dependency was most discernable near the hMSCs frequency transition region from $\mathrm{nDEP}$ to $\mathrm{pDEP}$ and the 
$f_{x o}$ value, both were encompassed in the range of 0.61 and 1.4 MHz. Maxwell-Wagner interfacial polarization mechanisms dominate in the frequency range from $0.010 \mathrm{MHz}$ to $10 \mathrm{MHz}$ in the $\beta$-dispersion region [40] such that an observed $f_{x o}$ is influenced by the ionic interactions of the hMSCs membrane with the surrounding medium. As the frequency increases in the $\mathrm{MHz}$ range, this interfacial polarization transitions to being dominated by the membrane permittivity, which was more consistent with the optimized model parameters in Table 4.1 . Thus at $0.10 \mathrm{~S} / \mathrm{m}$, the hMSCs membrane was electrically more permissive to the ions driven by the AC field evidenced by the shift in the DEP spectra to higher frequencies. These experimental findings were consistent with the core-shell DEP polarization model optimization whereby the cytoplasm properities did not vary, but the membrane parameters did (Table 4.1 summary). These experimental observations were corroborated with increases in the calculated membrane capacitance, $2.2 \mathrm{pF}$ and $4.5 \mathrm{pF}$ at $0.030 \mathrm{~S} / \mathrm{m}$ and $0.10 \mathrm{~S} / \mathrm{m}$, respectively. Small deviations between the experimental data and the core-shell spherical DEP polarization model likely occurred because the model does not account for all of the hMSCs morphological and biosurface marker complexities.

To reduce the hMSCs morphological complexity, the cells were treated with ELP-PEI to yield a uniform spheroidal cell shape. The ELP-PEI treatment concurrently caused some hMSCs to form spheroidal aggregates consistent with previous work [46, 47]. For low frequencies, $0.010-0.10 \mathrm{MHz}$, untreated and ELPPEI treated hMSCs similarly exhibited nDEP, suggesting comparable membrane resistance at those frequencies. Above $0.10 \mathrm{MHz}$, ELP-PEI treated hMSCs only 
exhibited nDEP behavior, which deviated from untreated hMSCs that transitioned to pDEP behavior. There are a few possible scenarios to explain this behavior: (1) the ELP-PEI may be incorporating itself into the hMSCs membrane, (2) small concentrations of ELP-PEI could be present in the dextrose solution surrounding the cells, and/or (3) cells aggregating into spheroids could shield membrane polarization effects.

Within hypothesis (1) context, that ELP-PEI may have intercolated into the hMSCs membrane, the core-shell spherical DEP polarization model key parameters in Figure 4.6 and Table 4.1 illustrate that decreases in hMSCs membrane permittivity were necessary to match experimental data $\left(\varepsilon_{\mathrm{mem}}\right.$ is 0.050$)$. A decrease in cytoplasm conductivity from $0.63 \mathrm{~S} / \mathrm{m}$ to $6.3 \times 10^{-3} \mathrm{~S} / \mathrm{m}$ also supports this claim (Figure $4.7 \mathrm{~d}$ ), but since the DEP tests were completed in the $\beta$-dispersion region, its unlikely that the DEP responses recorded were representative of the cytoplasm structure inside of hMSCs.

Because the ELP-PEI treatment caused hMSCs to form spheroidal aggregates, size effects were examined. Using the treated hMSCs starting model parameters (Table 4.1) in the core-shell spherical DEP polarization model, the size would have to reduce to $0.75 \mu \mathrm{m}$ to exhibit $\mathrm{nDEP}$ behavior similar to the observed experimental data. This was not feasible for the treated cells and their aggregates, which had average sizes of $17 \pm 4 \mu \mathrm{m}$ and $43 \pm 16 \mu \mathrm{m}$, respectively. The increased size of the treated hMSC aggregates would increase the exerted DEP force. However, there was no evidence that this size increase would prevent interrogation of biosurface markers present on 
hMSCs membranes. Data did show strong media/membrane polarization behaviors, which are prerequisites for surface biosurface marker-induced stem cell separations in nonlinear AC electric fields. We conclude that the ELP-PEI treatment did achieve standardization of the hMSCs morphology and simultaneously altered DEP behavior.

\subsection{Conclusions}

hMSCs are an important cell system due to their differentiation capacity, trophic activity, and their ability to self-renew. Their therapeutic potential is currently being explored through clinical trials to treat lupus, Crohn's disease, and diabetes mellitus. For successful and effective stem cell therapies, hMSCs purification after bone marrow isolation could be considerably improved to increase selective recovery of the most highly viable cells. Separation techniques like FACS and MACS are expensive and alter cellular function and viability due to antigen tagging. Userfriendly DEP microdevices have previously demonstrated rapid cell separation and no loss in cell viability with low voltage AC electric fields [37]. hMSCs populations are heterogeneous with varied morphology, so the development of a continuous cell sorting microdevice needs to concurrently control for and accurately measure (1) hMSCs dielectric signatures and (2) cell morphology variations. The later was accomplished in this work by morphologically standardizing the cell population with ELP-PEI. Additionally, hMSC population heterogeneity correlates with biosurface marker expression predisposing cells for adipocytes, chondrocytes, and osteoblasts. Thus, after morphological standardization, precise measurements of DEP dielectric signatures may facilitate biosurface marker-dependent cell separations. 
hMSCs have a distinct dielectric dispersion defined by internal structures, cell membrane and cytoplasm. hMSCs membrane mostly affects the DEP response at radio frequencies in the $\beta$-dispersion region; the radio frequencies polarize the hMSCs surface, facilitating a DEP force along the field gradient, and thus allowing interogation of the cell membrane dielectric properties. The DEP results in this work reveal that the conductivity of hMSCs suspending solution is critical to the membrane polarization. Higher conductivity solutions increase the membrane permittivity shifting the DEP spectra to higher frequencies for $0.10 \mathrm{~S} / \mathrm{m}$ than $0.030 \mathrm{~S} / \mathrm{m}$. This translates to a membrane capacitance increase from $2.2 \mathrm{pF}$ for $0.030 \mathrm{~S} / \mathrm{m}$ to $4.5 \mathrm{pF}$ for $0.10 \mathrm{~S} / \mathrm{m}$. Similarly, the membrane permittivity increases from 2.0 for $0.030 \mathrm{~S} / \mathrm{m}$ to 4.1 for $0.10 \mathrm{~S} / \mathrm{m}$. The solution conductivity dependence was also apparent at the $f_{x o}$ for hMSCs; $0.030 \mathrm{~S} / \mathrm{m}$ yields a lower $f_{x o}$ at $0.62 \mathrm{MHz}$ and $0.10 \mathrm{~S} / \mathrm{m}$ yields a lower $f_{x o}$ at 1.3MHz. Frequency ranges tested did not enable measurement of the higher $f_{x o}$. This dielectric signature is unique for hMSCs because typical low $f_{x o}$ 's for other cell systems fall in the range of $0.010-0.10 \mathrm{MHz}$ [44]. These differing DEP spectra could be harnessed for hMSC cell separations from these other cell systems. Complicating any separation endeavor is that the untreated hMSC DEP responses at static frequencies varied within a single culture, thus reproducible separations need to consider and control for biosurface marker expression.

The ELP-PEI treatment successfully standardized hMSCs population morphology, although spheroidal cell aggregates were concurrently observed. The ELP-PEI treatment concurrently increased the DEP response reproducibility. This 
standardization occurred because the polymer interacted with the cell membrane and shifted hMSCs polarization behaviors. Only nDEP responses were observed from 0.010-10MHz, which was substantially different from untreated hMSCs. The spheroidal cell aggregates present after ELP-PEI treatment should increase the DEP force, which is proportional to cell radius cubed. However, the strong media/membrane polarization behaviors observed suggest that this hMSC cell system is a good candidate for future surface biosurface marker-induced stem cell separations. With optimization of the ELP-PEI treatment, detection of molecular level differences in hMSCs may be realizable to aid understanding of biological functions and cell population purification for stem cell therapies.

\subsection{Conflict of Interest Statement}

The authors indicate no potential conflicts of interest.

\subsection{Acknowledgements}

The research completed in this paper was supported by Michigan Tech's Finishing Fellowship, Kings-Chavez-Parks Future Faculty Fellowship, and partially supported by the National Science Foundation (Award No: 1033525) to AVJ. The authors would like to thank Dr. Caryn Heldt for the use of her cell culture hood, and both Lijun Zhang and Qi Xing for assistance with hMSC samples. 


\subsection{Journal Article References}

1. G. Kolios and Y. Moodley, Respiration 85, 3 (2013).

2. A. Caplan, Journal of Cellular Physiology 213, 341 (2007).

3. A. Caplan and D. Correa, Cell Stem Cell 9, 11 (2011).

4. F. Zhao, JSM Biotechnology \& Biomedical Engineering 1, 1012 (2013).

5. S. Bobis, D. Jarocha and M. Majka, Folia Histochemica Et Cytobiologica 44, 215 (2006).

6. M. C. Stewart and A. A. Stewart, Veterinary Clinics of North America: Equine Practice 27, 243 (2011).

7. H. W. Wu, C. C. Lin and G. B. Lee, Biomicrofluidics 5, 013401 (2011).

8. H. K. Salem and C. Thiemermann, Stem Cells 28, 585 (2010).

9. F. Zhao, J. J. Veldhuis, Y. Duan, Y. Yang, N. Christoforou, T. Ma and K. W. Leong, Molecular Therapy 18, 1010 (2010).

10. F. Zhao, W. L. Grayson, T. Ma and A. Irsigler, Journal of Cellular Physiology 219, 421 (2009).

11. E. Fuchs and T. Chen, European Molecular Biology Organization 14, 39 ( 2013).

12. S. He, D. Nakada and S. J. Morrison, Annual Review of Cell and Development Biology 25, 377 (2009).

13. A. Caplan and J. E. Dennis, Journal of Cellular Biochemistry 98, 1076 (2006). 
14. L. Wu, H. J. Prins, M. N. Helder, C. A. van Blitterswijk and M. Karperien, Tissue Engineering: Part A 18, 1542 (2012).

15. B. Parekkadan and J. M. Milwid, Annual Review of Biomedical Engineering 12, 87 (2010).

16. G. P. Zheng, M. H. Ge, Q. Shu, M. Rojas and J. Xu, World Journal Pediatrics 9, 197 (2013).

17. A. R. Williams and J. M. Hare, Circulation Research 109, 923 (2011).

18. A. Jamnig and G. Lepperdinger, Candian Journal of Physiology and Pharmacology 90, 295 (2012).

19. G. Lanzoni, F. Alviano, C. Marchionni, L. Bonsi, R. Costa, L. Foroni, G. Roda, A. Belluzzi, A. Caponi, F. Ricci, P. L. Tazzari, P. Pagliaro, R. Rizzo, F. Lanza, O. R. Baricordi, G. Pasquinelli, E. Roda and G. P. Bagnara, Cytotherapy 11, 1020 (2009).

20. C. M. Kolf, E. Cho and R. S. Tuan, Arthritis Research \& Therapy 9, 204 (2007).

21. F. Pevsner, M., S. Levin and D. Zipori, Stem Cell Review and Reports 7, 560 (2011).

22. M. Gonzalez-Gonzalez, P. Vazquez-Villegas, C. Garcia-Salinas and M. RitoPalomares, Journal of Chemical Technology and Biotechnology 87, 2 (2012).

23. L. A. Flanagan, J. Lu, L. Wang, S. A. Marchenko, N. L. Jeon, A. P. Lee and E. Monuki, Stem Cells 26, 656 (2008). 
24. J. Vykoukal, D. M. Vykoukal, S. Freyberg, E. U. Alt and P. R. C. Gascoyne, Lab on a Chip 8, 1386 (2008).

25. F. Mosna, L. Sensebe and M. Krampera, Stem Cells and Development 19, 1449 (2010).

26. P. Mafi, S. Hindocha, R. Mafi, M. Griffin and W. S. Khan, The Open Orthopaedics Journal 5, 253 (2011).

27. F. Haasters, W. C. Prall, D. Anz, C. Bourquin, C. Pautke, S. Endres, W. Mutchler, D. Docheva and M. Schieker, Journal of Anatomy 214, 759 (2009).

28. R. Pethig, A. Menachery, S. Pells and P. De Sousa, Journal of Biomedicine and Biotechnology 182581 (2010).

29. C. Zhang, K. Khoshmanesh, A. Mitchell and K. Kalantar-zadeh, Analytical and Bioanalytical Chemistry 396, 401 (2010).

30. K. M. Leonard and A. R. Minerick, Electrophoresis 32, 2512 (2011).

31. S. K. Srivastava, P. R. Daggolu, S. C. Burgess and A. R. Minerick, Electrophoresis 29, 5033 (2008).

32. A. Salmanzadeh, H. Kittur, M. B. Sano, P. C. Roberts, E. M. Schmelz and R. V. Davalos, Biomicrofluidics 6, 024104 (2012).

33. A. Salmanzadeh, L. Romero, H. Shafiee, R. C. Gallo-Villanueva, M. A. Stremler, S. D. Cramer and R. V. Davalos, Lab on a Chip 12, 182 (2012).

34. K. Khoshmanesh, S. Nahavandi, S. Baratchi, A. Mitchell and K. Kalantarzadeh, Biosensors \& Bioelectronics 26, 1800 (2011). 
35. S. Patel, D. Showers, P. Vedantam, T. R. Tzeng, S. Z. Qian and X. C. Xuan, Biomicrofluidics 6, 034102 (2012).

36. M. A. A. Razak, K. F. Hoettges, H. O. Fatoyinbo, F. H. Labeed and M. P. Hughes, Biomicrofluidics 7, 064110 (2013).

37. J. Lu, C. A. Barrios, A. R. Dickson, J. L. Nourse, A. P. Lee and L. A. Flanagan, Integrative Biology 4, 1223 (2012).

38. A. Y. Wu and D. M. Morrow, Journal of Translational Medicine 10, 99 (2012).

39. R. Pethig, Biomicrofluidics 4, 022811 (2010).

40. T. N. G. Adams, K. M. Leonard and A. R. Minerick, Biomicrofluidics 7, $064114(2013)$.

41. Z. R. Gagnon, Electrophoresis 32, 2466 (2011).

42. B. G. Hawkins, C. Huang, S. Arasanipalai and B. J. Kirby, Analytical Chemistry 83, 3507 (2011).

43. M. Alshareef, N. Metrakos, E. J. Perez, F. Azer, F. Yang, X. Yang and G. Wang, Biomicrofluidics 7, 011803 (2013).

44. A. Salmanzadeh, E. S. Elvington, P. C. Roberts, E. M. Schmelz and R. V. Davalos, Integrative Biology 5, 843 (2013).

45. P. R. C. Gascoyne, S. Shim, J. Noshari, F. F. Becker and K. Stemke-Hale, Electrophoresis 34, 1042 (2013).

46. P. A. Turner, L. M. Harris, C. A. Purser, R. C. Baker and A. V. Janorkar, Biotechnology and Bioengineering 111, 174 (2014). 
47. A. V. Janorkar, L. M. Harris, B. S. Murphey and B. L. Sowell, Biotechnology and Bioengineering 108, 1171 (2011).

48. L. Rozitsky, A. Fine, D. Dado, S. Nussbaum-Ben-Shaul, S. Levenberg and G. Yossifon, Biomedical Microdevices 15, 859 (2013).

49. S. Cho and H. Thielecke, Microelectronic Engineering 85, 1272 (2008).

50. A. Di Biasio and C. Cametti, Colloid and Surfaces B: Biointerfaces 84, 433 (2011).

51. Z. Gagnon, J. Gordon, S. Sengupta and H. C. Chang, Electrophoresis 29, $2272(2008)$.

52. K. Zhao, W. Bai and H. Mi, Bioelectrochemistry 69, 49 (2006).

\subsection{Chapter Reference}

1. Adams, T,N.G., Turner, P.A., Janorkar, A.V., Zhao, F., and Minerick, A.R., Biomicrofluidics, submitted, 2014. 


\section{Chapter 5 Frequency Sweep Rate Dependence on the}

\section{Dielectrophoretic Response of Polystyrene Beads and Red}

\section{Blood Cells [1]}

${ }^{1}$ T. N. G. Adams, K. M. Leonard, and A. R. Minerick

\subsection{Abstract}

Alternating current (AC) dielectrophoresis (DEP) experiments for biological particles in microdevices are typically done at a fixed frequency. Reconstructing the DEP response curve from static frequency experiments is laborious, but essential to ascertain differences in dielectric properties of biological particles. Our lab explored the concept of sweeping the frequency as a function of time to rapidly determine the DEP response curve from fewer experiments. For the purpose of determining an ideal sweep rate, homogeneous $6.08 \mu \mathrm{m}$ polystyrene (PS) beads were used as a model system. Translatability of the sweep rate approach to $\sim 7 \mu \mathrm{m}$ red blood cells (RBC) was then verified. An $\mathrm{Au} / \mathrm{Ti}$ quadrapole electrode microfluidic device was used to separately subject particles and cells to $10 \mathrm{Vpp} \mathrm{AC}$ electric fields at frequencies ranging from $0.010-2.0 \mathrm{MHz}$ over sweep rates from 0.00080 to $0.17 \mathrm{MHz} / \mathrm{s}$. PS beads exhibited negative DEP assembly over the frequencies explored due to MaxwellWagner interfacial polarizations. Results demonstrate that frequency sweep rates must

\footnotetext{
${ }^{1}$ The material contained in this chapter was previously published in Biomicrofluidics, 2013. 7: p. 064114.
} 
be slower than particle polarization timescales; sweep rates near $0.00080 \mathrm{MHz} / \mathrm{s}$ yielded DEP behaviors very consistent with static frequency DEP responses for both PS beads and RBCs.

Key Words: Dielectrophoresis, frequency sweep, polystyrene beads, red blood cells, interfacial polarization, microfluidic devices

\subsection{Introduction}

Microfluidic and dielectrophoretic (DEP) technologies enable a wide variety of particle polarizations with nonuniform electric fields on microchips $[2,3]$ to achieve particle manipulation, concentration, separations, and property-based identification. Particles [4-6] include bioparticles (DNA [7], virus [8], protein [9]) as well as cells (blood cell types $[10,11]$, cancer [12-14], stem cells [15], and yeast [16]). The advantages to coupling DEP with microfluidics are small sample size (on the order of microliters), rapid analysis (approximately minutes to achieve results), minimal sample preparation, and minimal waste production $[17,18]$. Traditionally, DEP experiments are completed at static, fixed frequencies such that maximum particle polarization can be achieved and measured. Multiple experiments are conducted, each at discrete frequencies over the range of interest to stitch together DEP response spectra; this is a labor-intensive approach. Further disadvantages are that extended field exposure times at fixed frequencies can change particle properties [15] or cell viability [19]. Prior research has used rapid frequency sweeping to increase cell separation efficiency [20-24], but to the best of our knowledge, frequency sweeping 
has not been explored to generate continuous DEP spectra. Therefore, a detailed study examining the effects of frequency sweeping on particle polarization is pertinent and timely. In this paper, we demonstrate that frequency can be swept with time in the $\beta$ dispersion region thus enabling interrogation of cells at multiple frequencies within a short time period. The benefits of using a frequency sweep technique are that nearly continuous DEP response curves, when coupled with automated response analysis, can be compiled in near real time and the number of experiments needed to obtain particle DEP spectra are greatly reduced.

DEP enables phenotypically similar biological cells to be discriminated based on dielectric properties including the conductivity and permittivity of the membrane, cytoplasm, and other structurally relevant organelles. Cell components and structure contribute to a cell's signature dielectric dispersion $[3,16]$. A particle's complex permittivity is frequency dependent and characterized by dielectric dispersion regions $\left(\gamma, \beta\right.$, and $\alpha$, where $\left.\omega_{\alpha}<\omega_{\beta}<\omega_{\gamma}\right)$ specific to an applied frequency. This work explores 0.010 to $2.0 \mathrm{MHz}$ in the $\beta$-dispersion region [25] because the Clausius-Mossotti factor, which governs sign and polarization strength, for polystyrene beads is nearly constant over this range. Maxwell-Wagner theory describes the polarization mechanism of particles in the $\beta$-dispersion region as interfacial polarization where moving charges build around the interface of a charged or charge-neutral particle and exchange ions with the suspending medium (ref. [26], pp.33-38). Interfacial particle polarization creates an induced dipole moment such that the particle experiences disproportionate 
forces in each half cycle of the alternating current (AC) field resulting in net particle movement (ref. [26], pp. 8-11).

Polarized particles can exhibit either positive dielectrophoresis ( $\mathrm{pDEP}$ ) or negative dielectrophoresis (nDEP) as a consequence of the frequency-dependent polarizability of the particle in the surrounding medium (ref.[26], p.10) [27]. Particles that exhibit pDEP move to high electric field regions and particles that exhibit nDEP move to low electric field regions $[3,16]$. This motion up and down electric field gradients is described by the Clausius-Mossotti factor for spherical particles [16]

$$
\begin{aligned}
& f_{c m}=\frac{\widetilde{\varepsilon}_{p}-\widetilde{\varepsilon}_{m}}{\widetilde{\varepsilon}_{p}+2 \widetilde{\varepsilon}_{m}}, \\
& \widetilde{\varepsilon}_{i}=\widetilde{\varepsilon}_{i}+\frac{\sigma_{i}}{\omega j},
\end{aligned}
$$

where $\tilde{\boldsymbol{\varepsilon}}_{\boldsymbol{i}}$ is the complex permittivity of the particle $(\mathrm{i}=\mathrm{p})$ and of the medium $(\mathrm{i}=\mathrm{m})$, which are both functions of conductivity $(\sigma)$, permittivity $(\varepsilon)$, and angular frequency $(\boldsymbol{\omega})[26]$.

Polarization is not an instantaneous event [28]; charge transport into the interface takes a few microseconds in response to the electric field. Maxwell-Wagner dielectric relaxation is a physical phenomena related to the transport delay of cation and anion alignment in and around the interface of the dielectric particle [29]. At lower frequencies $(<\sim 10 \mathrm{MHz})$, particle polarization is driven by this conductive polarization. At higher AC frequencies, charges do not have enough time to move into and around the interface double layer, so particles experience polarization lag time as 
a result of the rapidly modulating field and do not reach maximum polarization. Maxwell-Wagner dielectric relaxation is characterized by a time constant, $\tau_{\mathrm{MW}}$, which is unique to each particle or cell due to the time constant's dependence on the cell dielectric properties. The time required for a particle to reach maximum polarization given by (ref. [26] ,p. 27) [25, 30]

$$
\tau_{T W}=\frac{\left(\varepsilon_{p}+2 \varepsilon_{m}\right) \varepsilon_{0}}{\sigma_{p}+2 \sigma_{m}}
$$

Typical relaxation times for particle polarization vary from pico to microseconds (ref. [26],p. 37) [25, 30], and the calculated $\tau_{\mathrm{MW}}$ for polystyrene (PS) beads in our Epure $\mathrm{H}_{2} \mathrm{O}$ medium at $2.5 \times 10^{-4} \mathrm{~S} / \mathrm{m}$ is $3.5 \mu$ s. Thus, a single $\mathrm{AC}$ cycle is on the order of 0.01 to $2 \mu \mathrm{s}$; the time delay in ion transport within a static frequency field of 0.010 to 2.0 $\mathrm{MHz}$ is such that 2 to $350 \mathrm{AC}$ cycles must be completed before the particle experiences full polarization.

The Maxwell-Wagner dielectric timescales for charge transport into and around the interface becomes important when the frequency is swept, i.e. changes as a function of time. Figure 5.1a crudely cartoons the Maxwell-Wagner particle polarization at the interface under static frequency as well as slow and fast frequency sweep rates. At a static frequency in the $\beta$-dispersion region, the particle experiences a constant frequency field such that the relaxation time is not a factor and the particle fully polarizes. A particle in a field with a slowly changing frequency sweep has relaxation time, $\tau_{\triangle \mathrm{FS}}$, that is less than $\tau_{\mathrm{MW}}$ and thus the particle interface fully polarizes. In contrast, a particle in a fast frequency sweep has a relaxation time, $\tau_{\Delta \mathrm{FS}}$, that is larger 
than $\tau_{\mathrm{MW}}$ and the particle interface does not have time to fully polarize in the field. PS beads are lossy dielectric particles treated as homogeneous spheres and are thus an idealized particle to examine new techniques, devices or approaches to dielectrophoretic characterizations. Our system is easily able to discern pDEP and nDEP transitional behavior and adaptable to our new frequency sweep technique. The homogeneous spherical DEP polarization model for PS beads $\left(\varepsilon=2.5\right.$ and $\sigma=9.4 \times 10^{-}$ ${ }^{5} \mathrm{~S} / \mathrm{m}$ ) suspended in Epure $\mathrm{H}_{2} \mathrm{O}$ displays only nDEP behavior over 0.010 to $2.0 \mathrm{MHz}$. In this study, dielectrophoretic responses of PS beads (model system) were quantified at both static frequencies and frequency sweeps at rates ranging from 0.00080 to $0.17 \mathrm{MHz} / \mathrm{s}$ over the $\beta$-dispersion frequency range of $0.010-2.0 \mathrm{MHz}$ in Epure $\mathrm{H}_{2} \mathrm{O}$ medium at $2.5 \times 10^{-4} \mathrm{~S} / \mathrm{m}$. At these experimental conditons $\mathrm{AC}$ electroosmosis, normally at $100-500 \mathrm{kHz}$, and electrothermal flow, normally occurs in high conducitivity media $1 \mathrm{~S} / \mathrm{m}$ or greater, was not a factor [31]. PS bead motion in the electric field was imaged with video microscopy and analyzed using three techniques: intensity profiles, transient response, and particle velocities. Image intensity analysis has been used by other researchers to quantify the pDEP and nDEP behavior of particles representing particle concentration [32], voltage trapping [33], cell counting [34], and noncontinuous DEP spectra $[35,36]$. Our intensity analysis method is used to develop a continuous DEP response spectra and data shows that frequency sweep rates impact particle polarization due to dielectric relaxation limitations. This frequency sweep technique was further extended to negatively 
charged biconcave red blood cells (RBCs), which are an important cellular system for medical disease diagnostics [37-39].

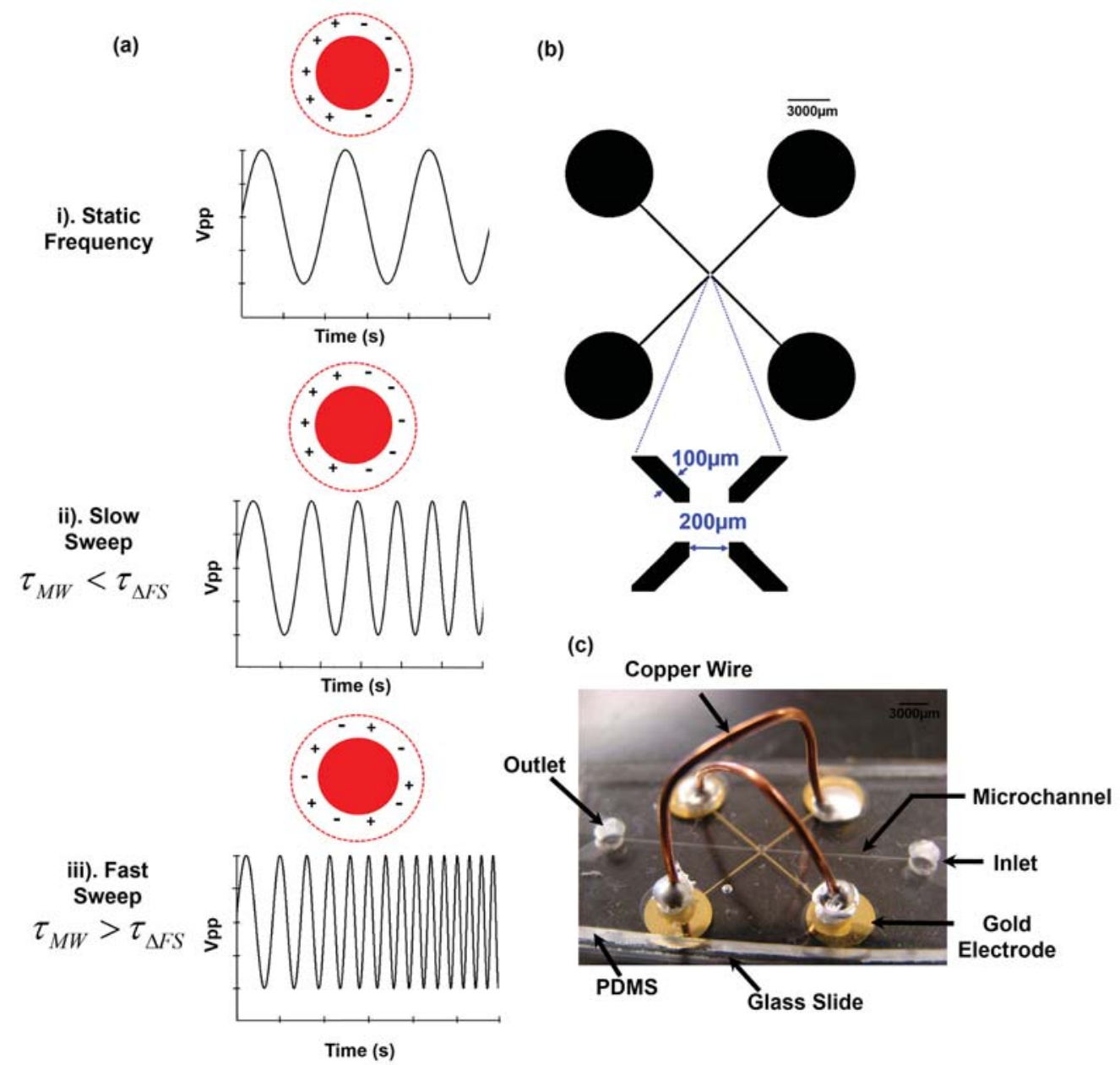

Figure 5.1 (a) Dielectric relaxation mechanism for PS beads showing cases when i) particle polarization occurs at a static frequency, ii) $\tau_{\mathrm{MW}}$ is shorter than the slow frequency sweep rate $\left(\tau_{\Delta \mathrm{FS}}\right)$ allowing the bead interface time to polarize in response to the nonuniform $\mathrm{AC}$ field, and iii) $\tau_{\mathrm{MW}}$ is longer than the $\tau_{\Delta \mathrm{FS}}$ for fast frequency sweep rates and the bead interface does not have time to fully polarize. (b) Schematic of the 
quadrapole electrodes micro patterned onto a glass slide, and (c) microdevice with PDMS fluidic layer bonded above the quadrapole electrodes silver epoxies to copper leads.

\subsection{Materials and Methods}

The microdevice shown in Figure 5.1c was fabricated according to previously published microfabrication techniques [7], with the $100 \mu \mathrm{m}$ wide electrodes spaced $200 \mu \mathrm{m}$ apart aligned at $90^{\circ}$ along the bottom of a $70 \mu \mathrm{m}$ deep by $1000 \mu \mathrm{m}$ wide microfluidic chamber as shown in Figure 5.1b. Polystyrene beads (Cat No. PP-60-10, Spherotech, Lake Forest, IL, USA), $6.08 \mu \mathrm{m}$ in diameter were centrifuged at $1300 \mathrm{~min}^{-}$

${ }^{1}$ for 5 mins to separate the beads from the liquid. The PS beads were resuspended in Epure $\mathrm{H}_{2} \mathrm{O}\left(18 \mathrm{M} \Omega\right.$ or $\left.2.5 \times 10^{-4} \mathrm{~S} / \mathrm{m}\right)$ at a $1: 10$ (bead to water) volumetric dilution ratio and vortexed. Microdevice was pre-rinsed with Epure $\mathrm{H}_{2} \mathrm{O}$ and Alconox precision cleaner (Cat No. 1104, Alconox Inc, White Plains, NY, USA) to prevent bead adhesion. PS bead-Epure $\mathrm{H}_{2} \mathrm{O}$ suspension was pumped to the microchamber using a syringe. Time was allowed for inlet and outlet pressures to equalize and flow to stop. The function generator (Agilent 33250A, Agilent, Santa Clara, CA, USA) was connected via copper leads to produce a 10Vpp AC sine wave with frequencies ranging from $0.010-2.0 \mathrm{MHz}$ at specific frequency sweep rates $0.00080,0.0011$, $0.0030,0.0063,0.013,0.021,0.028,0.042,0.056,0.083$, and $0.17 \mathrm{MHz} / \mathrm{s}$. Frequency sweeps linearly increased the applied frequency as a function of time. Greater than five $(n>5)$ static frequency experiments were completed at each frequency 0.010, 
$0.020,0.030,0.040,0.050,0.20,0.40,0.60,0.80,1.0,1.2,1.4,1.6,1.8$, and $2.0 \mathrm{MHz}$ by applying $10 \mathrm{Vpp}$ for $90 \mathrm{~s}$. These DEP static frequency responses were compared to each frequency sweep rate DEP responses. For the static and frequency sweep experiments, the PS bead concentration was between $238-263$ beads in the $t=0$ field of view. Video recordings of experiments were taken at $30 \mathrm{fps}$ at $640 \times 480$ pixels/image using LabSmith SVM Synchronized Video Microscope with a 10x objective (LabSmith, Livermore, CA, USA).

Video recordings of PS beads DEP behaviors were analyzed with ImageJ (NIH, Bethesda, MD) using intensity, transient slope, and velocity measurements. Since PS beads only exhibit nDEP over the frequency range of interest, intensity data acquisition from images was completed by drawing a rectangular box at the device center, ICTR, and background, IBK measured in a location with no PS beads present (See Figure 5.2a). ImageJ $Z$ Project function was used to average the pixel intensities in the specified boxed region. The initial background, $\mathrm{I}_{\mathrm{BK}}(\mathrm{t}=0)$ and center intensity, $\mathrm{I}_{\mathrm{CTR}}(\mathrm{t}=0)$ were subtracted from the center and background intensity at each time, $I_{C T R}(t)$ and $I_{B K}(t)$, and then a normalized intensity was calculated by dividing by the maximum intensity experienced by the PS beads,

$$
\bar{I}_{D E P, t}=\frac{\left[\left(I_{C T R}-I_{B K}\right)_{t}+\left(I_{B K}-I_{C T R}\right)_{t=0}\right]}{\left[\left(I_{C T R}-I_{B K}\right)_{t}+\left(I_{B K}-I_{C T R}\right)_{t=0}\right]_{M A X}}
$$

This normalized intensity tracked the real-time magnitude of the PS bead DEP response, which had two distinct regions: transient where beads moved with nDEP toward the center, and steady-state (SS) where beads achieved tight packing at the 
device center. These two responses were analyzed separately via transient slope and particle velocity.

The transient response of the PS beads was extracted from the steady-state response via signal processing in which the delay and rise time were quantified. The PS bead delay time, $t_{d}$, was characterized as the time required for the intensity response to reach $50 \%$ of the final intensity response for the first time. The rise time, $\mathrm{t}_{\mathrm{r}}$, was determined as the time needed for the intensity response to reach $100 \%$ of the final intensity response for the first time (ref. [40], pp. 517-518). This allowed the transient response to be segmented and a linear trend line was fit between $t_{d}$ and $t_{r}$ where $t_{d}<t_{r}$. A comparison of the transient slope for frequency sweep rates and static frequency measurements is given in Figure 5.3c. PS bead velocities were determined from the original video by tracking the $\mathrm{x}-$, y-pixel position of individual PS beads from 0-50s. PS bead located within $5 \mu \mathrm{m}$ of electrode tips were selected to control for similar electric field gradients. This procedure was repeated for at least 10 beads in each specific frequency sweep rate and static experimental video.

For the experiments involving human $\mathrm{RBCs}$, blood type $\mathrm{O}+$ was obtained from a single donor and centrifuged at $1400 \mathrm{rpm}$ for 5 mins to separate the packed RBCs from the plasma and leukocytes. The packed RBCs were removed, then resuspended at 1:75 v:v in $0.10 \mathrm{~S} / \mathrm{m}$ isotonic dextrose buffer doped with $0.75 \%$ BSA (Cat No. A7906, Sigma Aldrich, St. Louis, MO, USA) to prevent cell/device adhesion. This $\mathrm{RBC}$ suspension was syringe-pumped to the microchamber, time allowed for flow to stop and the $10 \mathrm{Vpp}$ signal applied over $0.010-0.50 \mathrm{MHz}$ (range reduced to avoid pDEP 
behavior) at frequency sweep rates of $0.00080,0.0063$ and $0.056 \mathrm{MHz} / \mathrm{s}(\mathrm{n}=7)$. RBC static frequency experiments were completed at $0.010,0.10,0.25$ and $0.50 \mathrm{MHz}$ at $10 \mathrm{Vpp}$ for $90 \mathrm{~s}(\mathrm{n}=7)$. Video microscopy at $25 \mathrm{x}$ and 1 fps was obtained with a Zeiss Axiovert Inverted Light Microscope (Zeiss, Germany). The video images were analyzed as described above for the PS beads.

\subsection{Results \& Discussion}

Frequency sweep rates ranging from 0.00080 to $0.17 \mathrm{MHz} / \mathrm{s}$ were explored to see if the nDEP response of PS beads would vary and/or correspond to static frequency measurements. The frequency range was chosen for the relatively consistent ClausiusMossotti factor, $\operatorname{Re}\left(f_{\mathrm{CM}}\right)$ for a homogeneous lossy polystyrene sphere of 0.26 to 0.48 (see Figure 5.2c) over the frequency range of 0.010 to $2.0 \mathrm{MHz}$. Static frequency experiments were completed at fixed values in this same frequency range. Figure 5.2a shows still images from both static frequency experiments and the frequency sweeps at $0.20,0.60$, and $1.0 \mathrm{MHz}$. For static frequencies, the response 45 seconds after field application is shown while for frequency sweeps of $0.0063,0.056$, and $0.17 \mathrm{MHz} / \mathrm{s}$, the image is shown at the time stamp when the specified frequency is reached. The electrodes are visible as black shadows in the images and the PS beads assemble due to $\mathrm{nDEP}$ forces at the central electric field gradient minima. Data was examined to determine the sweep rate that most closely approximated the static frequency response. Frequency sweeps 0.00080 and $0.0063 \mathrm{MHz} / \mathrm{s}$ (shown) tracked static frequency, or true, DEP responses while the slightly faster sweep of $0.056 \mathrm{MHz} / \mathrm{s}$ begins to lag the 
true DEP responses and at $0.17 \mathrm{MHz} / \mathrm{s}$ and faster, particles were unable to achieve sufficient polarization to respond sufficiently in the electric field.

nDEP responses were quantified via intensity analysis as described in materials and methods for all sweeps and all static frequency experiments. Figure 5.2b illustrates the frequency (and time) dependent intensity for the $0.0063 \mathrm{MHz} / \mathrm{s}$ sweep rate images shown in Figure 5.2a. This quantification of the PS bead nDEP response was correlated to total bead packing via the calibration shown in the inset. The 188bead count at the center deviates slightly from the initial, field off, bead count of 245 because PS beads also move down the electric field gradient to regions outside of the image field of view.
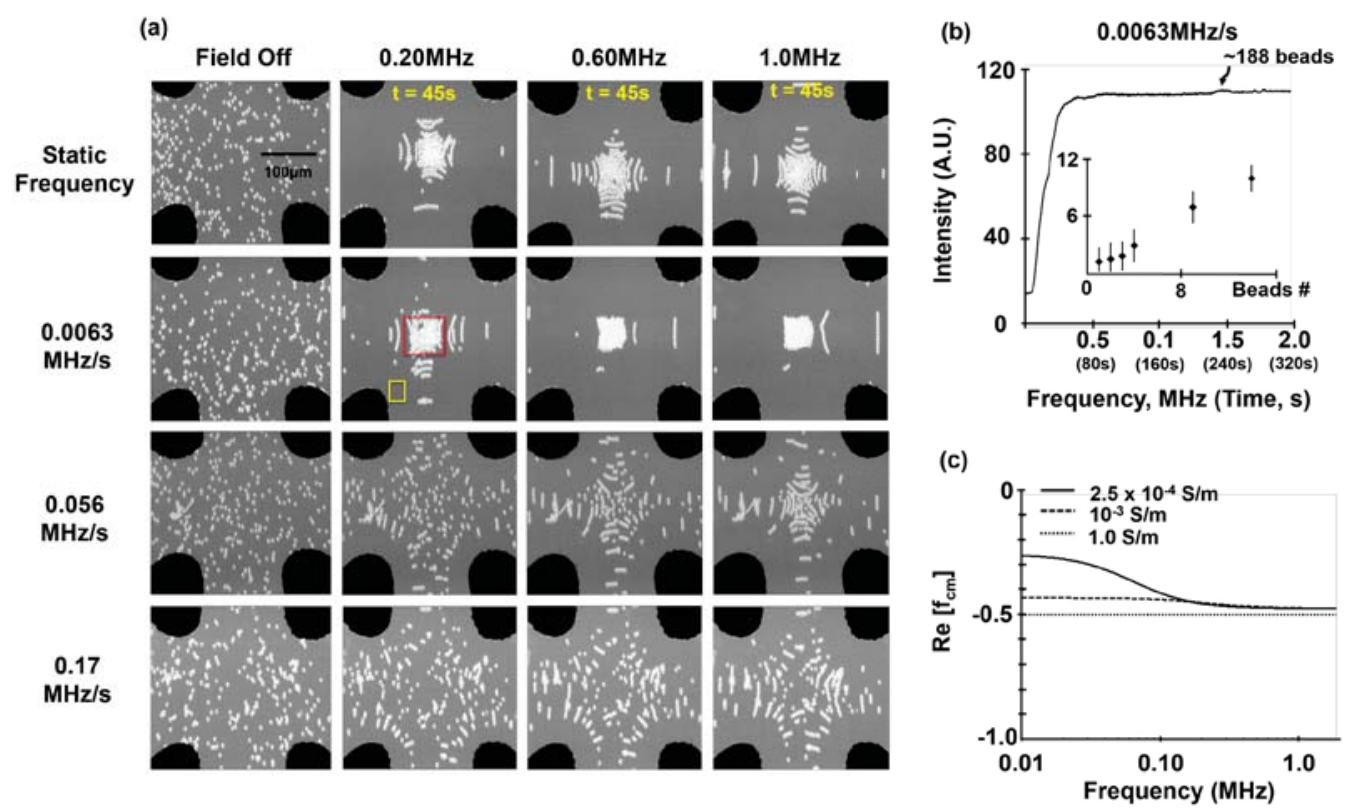

Figure 5.2 (a) nDEP behavior of $6 \mu \mathrm{m}$ PS beads suspended in E-pure $\mathrm{H}_{2} \mathrm{O} 2.5 \times 10^{-4}$ $\mathrm{S} / \mathrm{m}$ and $250 \mathrm{~V}_{\mathrm{pp}} / \mathrm{cm} 0.0063,0.056$ and $0.17 \mathrm{MHz} / \mathrm{s}$ sweep rates from $0.010 \mathrm{MHz}$ to 
1.0 MHz. (b) Raw intensity profile of PS beads in the center nDEP region (boxes shown at $0.20 \mathrm{MHz}$ ) at $0.0063 \mathrm{MHz} / \mathrm{s}$ sweep rate. Inset is a calibration of intensity per bead. (c) Clausius-Mossotti factor for the PS beads from $0.010 \mathrm{MHz}$ to $2.0 \mathrm{MHz}$ at three conductivities of $2.5 \times 10^{-4}, 1.0 \times 10^{-3}$, and $1.0 \mathrm{~S} / \mathrm{m}$. PS bead assembly at slower frequency sweep rates track static frequency responses while $0.056 \mathrm{MHz} / \mathrm{s}$ illustrates transitional behavior and frequency sweeps above $0.17 \mathrm{MHz} / \mathrm{s}$ substantially lag the true static frequency DEP responses.

Normalized intensities, Eq. (4), were compiled in Figure 5.3a for SS (i.e. 45 seconds) static frequency nDEP responses and $0.00080,0.0063,0.056 \mathrm{MHz} / \mathrm{s}$ frequency sweep rate nDEP responses. The time for sweep responses to achieve the true nDEP static response decreases as the sweep rate decreases. Frequency sweep rates 0.00080 and $0.0063 \mathrm{MHz} / \mathrm{s}$ are within the $95 \%$ confidence intervals $(n=7)$ of the static steady-state responses. Figure 5.3a inset shows that the slowest $0.00080 \mathrm{MHz} / \mathrm{s}$ sweep rate more quickly aligns closely with the static frequency responses. Figure $3 b$ compares average $0.0063 \mathrm{MHz} / \mathrm{s}(\mathrm{n}=8)$ to $0.17 \mathrm{MHz} / \mathrm{s}(\mathrm{n}=7)$ with the dashed lines signifying the upper and lower limits of the $95 \%$ confidence intervals for IDEP. The confidence intervals around the transient $0.0063 \mathrm{MHz} / \mathrm{s}$ sweeps are smaller than for $0.17 \mathrm{MHz} / \mathrm{s}$ over much of the frequency range indicating greater reproducibility at slower sweep rates. Faster sweep rates either do not reach SS or have a lag before reaching SS (compare to Figure 5.2a) suggesting the bead interface does not fully polarized and thus displays attenuated nDEP motion. 
The transient behavior was quantified for all static frequencies and frequency sweeps via a transient slope analysis as compiled in Figure 5.3c. Four static frequency measurements $0.010,0.60,1.0$ and $2.0 \mathrm{MHz}$ are shown compared to $0.00080,0.0063$, $0.028,0.056$, and $0.17 \mathrm{MHz} / \mathrm{s}$ frequency sweep rates. Static frequency transient slopes range between 0.023-0.095 and are within the $95 \%(\mathrm{p}<0.05)$ confidence intervals of $0.00080,0.0063$, and $0.028 \mathrm{MHz} / \mathrm{s}$ frequency sweep transient slopes. These slower sweep rates and $0.056 \mathrm{MHz} / \mathrm{s}$ differ at $\mathrm{p}<0.001$ from the fastest sweep rate of 0.17 $\mathrm{MHz} / \mathrm{s}$, which is also significantly different at $\mathrm{p}<0.001$ from the static measurements (except for $1.0 \times 10^{4} \mathrm{~Hz}$ with $\mathrm{p}<0.01$ ).

Individual bead velocities were compiled for static as well as frequency sweeps in Figure 5.3d. PS bead velocity corroborates the intensity profile and the slope analysis that $0.00080 \mathrm{MHz} / \mathrm{s}$ frequency sweep rate closely tracks the bead velocity at static frequencies. $0.056 \mathrm{MHz} / \mathrm{s}$ gives good estimations of static frequency bead velocity at times greater than $20 \mathrm{~s}$. Based on intensity, transient slope, and velocity analysis, the slow frequency sweep rate of $0.00080 \mathrm{MHz} / \mathrm{s}$ is most consistent with static frequency DEP responses. 
(a)

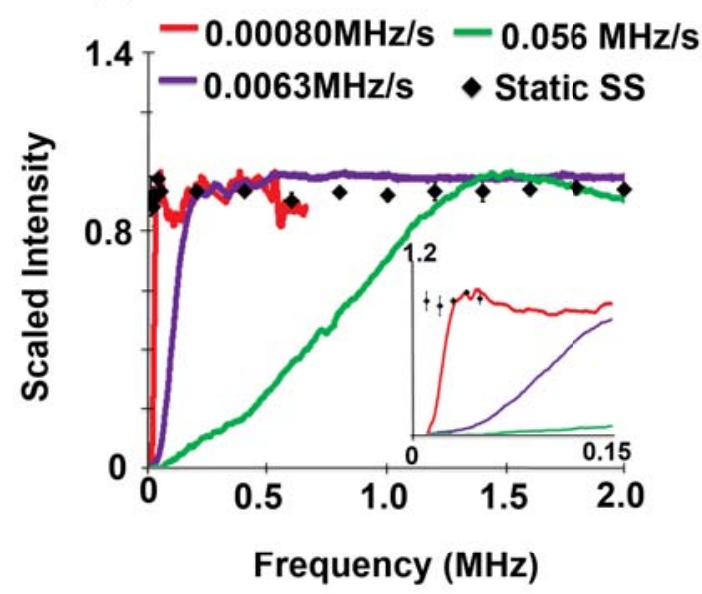

(c)

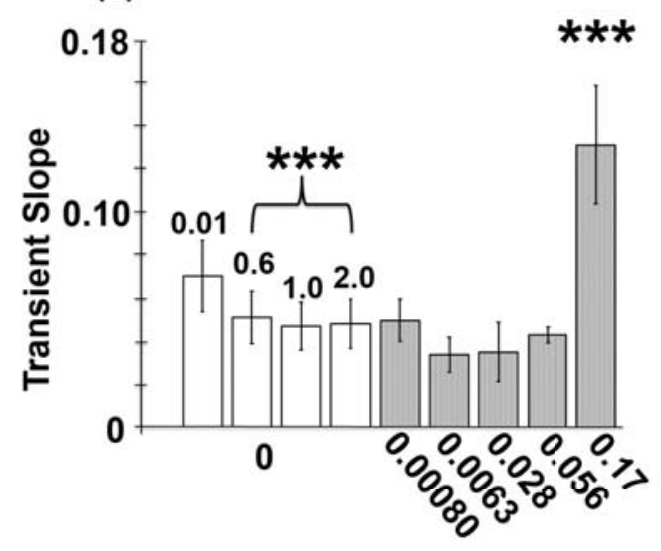

Frequency Sweep Rate (MHz/s) (b)

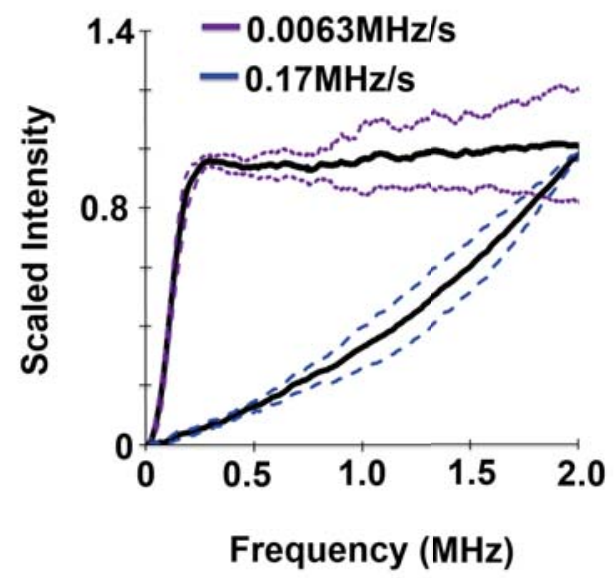

(d)

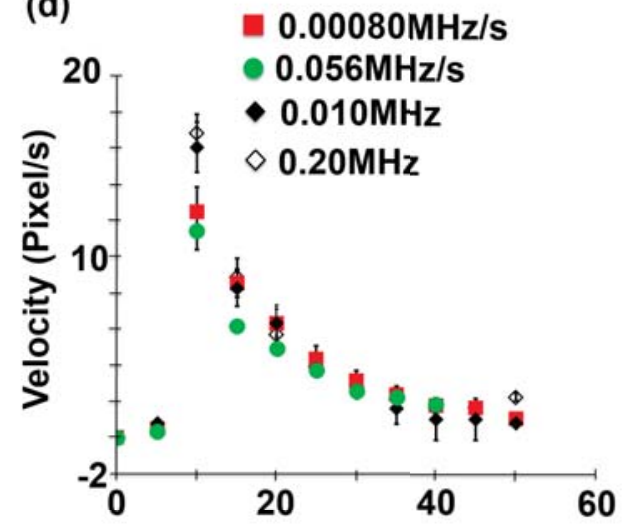

Time (s)

Figure 5.3 (a) $6 \mu \mathrm{m}$ PS beads nDEP intensity profiles for $0.00080,0.0063$, and 0.056 $\mathrm{MHz} / \mathrm{s}$ and static steady state (SS) measurements (black diamonds). Intensity analysis captures bead assembly to the quadrapole center with transient and SS regions. The slowest frequency sweep rate of $0.00080 \mathrm{MHz} / \mathrm{s}$ best predicts the static DEP responses. (b) Bead assembly profiles for $0.0063(\mathrm{n}=8)$ and $0.17 \mathrm{MHz} / \mathrm{s}(\mathrm{n}=7)$ with $95 \%$ confidence upper and lower limits shown as dashed lines. (c) Transient slope comparison for static frequencies $(0 \mathrm{MHz} / \mathrm{s})$ as well as frequency sweeps. (d) 
Comparison of static frequency and frequency sweep PS bead velocities from 0 to $50 \mathrm{~s}$. $0.00080 \mathrm{MHz} / \mathrm{s}$ results are consistently similar to the static frequency results.

There is an observable inverse relationship between the frequency sweep rate and particle polarization, slower sweep rates result in comparable particle polarization characteristics to static frequency responses. Dielectric relaxation is the driving force of this relationship; the calculated dielectric relaxation time Eq. (3) for PS beads in Epure $\mathrm{H}_{2} \mathrm{O}$ at $2.5 \times 10^{-4} \mathrm{~S} / \mathrm{m}$ is $3.5 \mu \mathrm{s}$, which corresponds to $\sim 0.28 \mathrm{MHz}$. There are two timescales that influence this behavior: the frequency itself and the change in frequency per time. The Maxwell-Wagner, conductivity-driven interfacial polarization mechanism occurs below $\sim 0.28 \mathrm{MHz}$; above this frequency threshold the interfacial polarization of the PS beads gradually decreases and the particle permittivity increasingly influences the DEP force. The experimental frequencies tested were within the range dominated by Maxwell-Wagner polarization such that maximum particle interfacial polarization was possible.

The second timescale of interest is the frequency change per time or frequency sweep rate, which determines how many consecutive cycles a particle experiences a specific frequency. At slower sweep rates, the PS beads experience a specific frequency for a large number of cycles and thus the beads have time to polarize because the timescale of the frequency change is slower than the dielectric relaxation time. A particle must experience a single frequency during the sweep for a minimum of $3.5 \mu \mathrm{s}$ for maximum interfacial polarization to be achieved. Upon polarization, the 
particle, which its current DEP force has to overcome inertia and Stokes drag to achieve observable particle motion down the electric field gradient. At static frequencies, it takes roughly $5 \mathrm{~s}$ for maximum velocity to be attained (see Figure 5.3d, AC field applied at $\mathrm{t}=5 \mathrm{~s}$ ) and as much as $45 \mathrm{~s}$ for final SS at the field gradient minima to be reached. As the sweep rate increases, the dielectric relaxation time and the rate of change of the frequency approach the same order of magnitude. Results suggest that $0.056 \mathrm{MHz} / \mathrm{s}$ is a transitional sweep rate because the DEP behavior roughly corresponds to the static behavior of the PS beads. With further increases in frequency sweep rates, the timescale for frequency change surpasses the dielectric relaxation timescale such that particles are unable to fully polarize resulting in an attenuated DEP response as shown with data in Figures 5.2, 5.3a, and 5.3b. Figure 5.3b also demonstrates that the transient behavior of the PS beads is more reproducible at slower frequency sweep rates, which can be attributed to the interfacial polarization timescale of the beads. Implications of the intensity, slope, and velocity analysis compared with static frequencies are that slow frequency sweep rates accurately predict the DEP response of PS beads because the changes in frequency are slower than the characteristic Maxwell-Wagner dielectric relaxation.

Thus, a frequency sweep approach can be utilized to attain accurate DEP behavior of PS beads, provided the sweep rate is slower than conductivity mediated interfacial polarization timescale. This result is reliable over frequency ranges where particle polarization is dominated by the conduction of free charges from the media. The charges are moving around the PS beads through the particle-liquid interface 
inducing a dipole, which causes PS bead movement down the electric field gradient to the electrode center. At different sweep rates the rate of movement of the charges varies which varies the rate of the dipole being induced, observed as dielectric relaxation. Each sweep rate has a unique dielectric relaxation time and our results are consistent with Maxwell-Wagner interfacial polarization theory. $0.00080 \mathrm{MHz} / \mathrm{s}$ is the optimal sweep rate necessary to predict the true DEP behavior of PS beads because it allows for full or partial (when the frequency is above $0.28 \mathrm{MHz}$ ) polarization.

Given that the sweep methodology yielded accurate DEP responses for the ideal system of PS beads, the same methodology and frequency sweep rates were explored with human RBCs. The three most successful PS bead frequency sweep rates were reproduced with human red blood cells: $0.00080 \mathrm{MHz} / \mathrm{s}, 0.0063 \mathrm{MHz} / \mathrm{s}$ and $0.056 \mathrm{MHz} / \mathrm{s}$. Static frequency experiments were also performed at $0.010 \mathrm{MHz}, 0.10$ $\mathrm{MHz}, 0.25 \mathrm{MHz}$ and $0.50 \mathrm{MHz}$. Seen in Figure 5.4a are 25x microscope images taken of the $t=45 \mathrm{~s}$ final static frequency frames aligned above the sweep time points that correspond to those four static frequencies. Qualitatively, the only sweep rate that accurately matches the static frequency behavior of the human RBCs is 0.00080 $\mathrm{MHz} / \mathrm{s}$. This behavior was further verified by the same intensity analysis as for PS beads. In Figure 5.4b, the scaled intensity is plotted for $0.00080,0.0063$ and 0.056 $\mathrm{MHz} / \mathrm{s}$ experiments $(\mathrm{n}=8)$ as compared to the static frequency intensities. After the initial 10s transition for the red blood cells to polarize and overcome drag, the slowest frequency sweep of $0.00080 \mathrm{MHz} / \mathrm{s}$ accurately predicts the static frequency behavior and is highly reproducible, with a very narrow 95\% confidence interval range (Figure 
5.4c). The fastest sweep rate of $0.056 \mathrm{MHz} / \mathrm{s}$ does not predict the static behavior of the human RBCs and is much less reproducible, as evidenced by the large $95 \%$ confidence interval in Figure 5.4c. From these experiments, we conclude that the optimal frequency sweep for determining the accurate DEP behavior of RBCs is $0.00080 \mathrm{MHz} / \mathrm{s}$. Due to the complex dielectric properties of cells, it is necessary to carefully compare frequency sweep rates with static frequency behaviors to ascertain optimal frequency sweep rates that accurately interrogate the cell of interest.
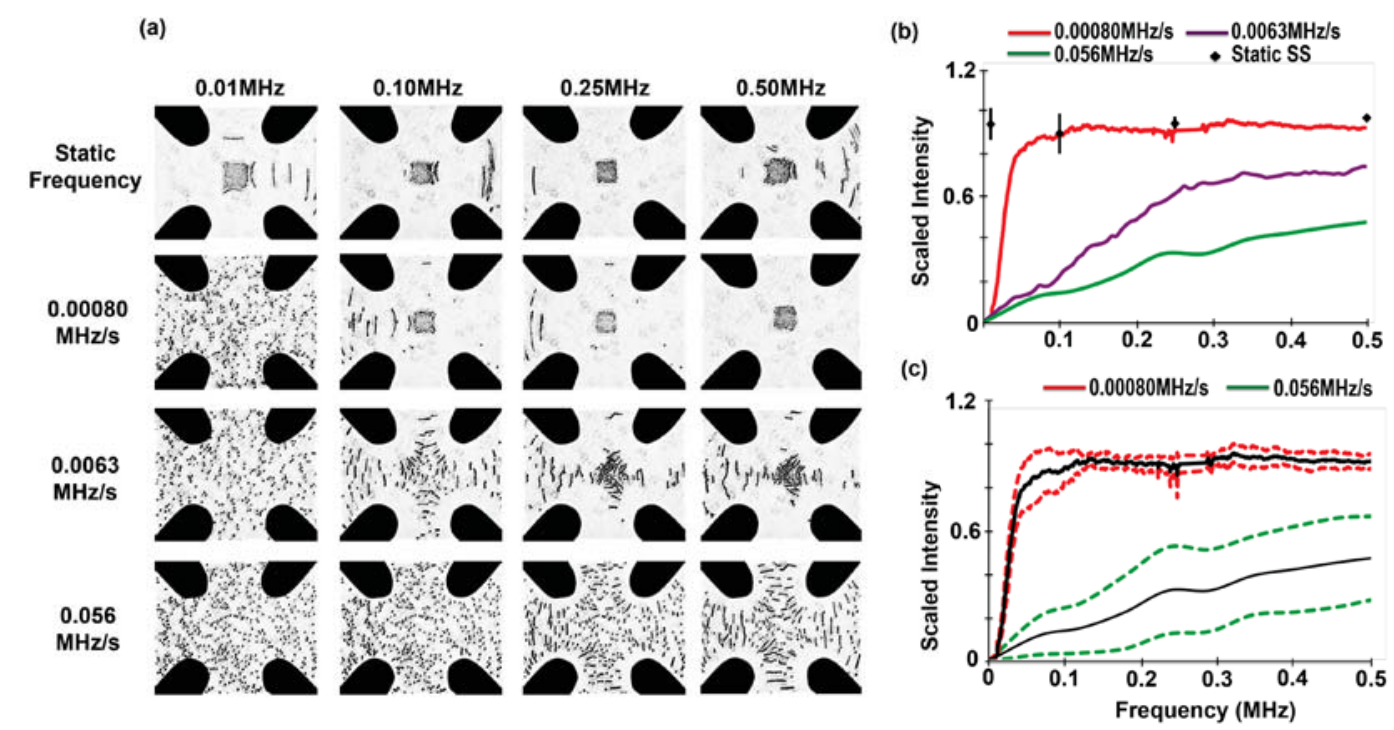

Figure 5.4 (a) nDEP behavior of RBCs suspended in $0.1 \mathrm{~S} / \mathrm{m}$ dextrose buffer and $250 \mathrm{~V} / \mathrm{cm}$ at $0.00080 \mathrm{MHz} / \mathrm{s}, 0.0063 \mathrm{MHz} / \mathrm{s}$ and $0.056 \mathrm{MHz} / \mathrm{s}$ sweep rates from 0.010 $\mathrm{MHz}$ to $0.50 \mathrm{MHz}$. (b) RBCs nDEP intensity profiles for $0.00080,0.0063$, and 0.056 $\mathrm{MHz} / \mathrm{s}$ and static measurements. (c) 0.00080 and $0.056 \mathrm{MHz} / \mathrm{s} \mathrm{RBC}$ assembly profiles $\mathrm{n}=8$, with $95 \%$ confidence interval upper and lower limits shown as dashed lines. 
The inverse relationship between the frequency sweep rate and particle polarization exists for RBCs. $\mathrm{RBC}$ dielectric relaxation is the driving force and the calculated dielectric relaxation time is $4.6 \mu$ s corresponding to $\sim 0.21 \mathrm{MHz}$. By choosing to sweep at a rate slower than this relaxation time the researchers are insured that the response during the sweep is the same as that at a static frequency. Further work is being done to explore the DEP response of RBCs when subjected to a wider range of sweep conditions.

\subsection{Conclusions}

Traditional DEP measurements are completed at single static frequencies in order to compile frequency by frequency, the DEP spectrum for a particle or cell system. This method is laborious, and as illustrated in this work, requires time for particles to fully polarize for accurate observed DEP responses. This work investigated the use of frequency sweeps as a means to more efficiently interrogate multiple frequencies in a single experimental run and systematically compared the responses to the nDEP response at fixed frequencies between 0.010 and $2.0 \mathrm{MHz}$. It was observed that frequency sweep rates influence the DEP response of PS beads and RBCs and further, the permissible frequency sweep rate is particle or cell dependent.

The underlying mechanism appears to be the same. At slower sweep rates, particles have more time to polarize in the electric field and therefore a more accurate and reproducible DEP spectrum can be obtained. At faster frequency sweep rates, the

particles are unable to achieve maximum interfacial polarization because of the 
dielectric relaxation time scale so the observed DEP response does not match the true DEP behavior of the particle.

For polystyrene beads at frequency sweep rates below $0.0063 \mathrm{MHz} / \mathrm{s}$, responses correlate closely with dielectric responses of particles subjected to a static frequency potential. In the PS bead system, $0.056 \mathrm{MHz} / \mathrm{s}$ is the transitional sweep rate where the particle dielectric relaxation is approximately the same order of magnitude as the shifts in frequency within the sweep. Dielectric responses continue to track the static frequency responses, although reproducibility is diminished. However as this sweep rate is increased further, conductivity dominated interfacial polarizations cannot be established and the PS bead frequency sweep data does not coincide with static frequency measurements.

For full utility in DEP experiments, this frequency sweep rate methodology must be translatable to cell systems. Results illustrated that only $0.00080 \mathrm{MHz} / \mathrm{s}$ accurately predicted the static frequency DEP responses of human RBCs. Red blood cells are substantially more morphologically and dielectrically complex than polystyrene beads. Calculation of the dielectric relaxation time, taking into account only the membrane permittivity and conductivity of 4.4 and $10^{-7} \mathrm{~S} / \mathrm{m}$, respectively [41] yields a dielectric relaxation time $\sim 4.6 \mu$ s roughly corresponding to $0.21 \mathrm{MHz}$. This relaxation time is larger than the PS bead relaxation time of $3.5 \mu \mathrm{s}$, so the optimal frequency sweep rate for red blood cells would be slower than that for PS beads. This result suggests that for each new cell system of interest it is imperative to determine the optimal frequency sweep rate to accurately and reproducibly interrogating the 
behavior of that cell. This work outlines a systematic technique to make comparisons between frequency sweep rate and static frequency shown. For all cell systems, sweep rates that are too fast will not allow the cell adequate time to polarize and will result in inaccurate and less reproducible DEP responses. An optimal frequency sweep rate can be estimated by calculating the Maxwell-Wagner dielectric relaxation time for the particle/cell of interest, provided the cell's permittivity and conductivity is known. The frequency sweep rate chosen for the DEP study should then remain at frequencies below the inverse dielectric relaxation time $\left(1 / \tau_{\mathrm{MW}}\right)$ for $5-45 \mathrm{~s}$ (longer times spent below the threshold give better DEP predictions). This is necessary because frequency sweep rates allow for particles/cells to be polarized incrementally with faster sweep rates, larger frequency step size, not allowing sufficient polarization time. Slower sweep rates, small frequency step size, allow particles to remain at lower frequencies for longer yielding full particle polarization consistent with static polarization measurements.

Since the cell's permittivity and conductivity are determined from the frequency dependent DEP spectrum, this presents a cyclical situation. However, this work has demonstrated that frequency sweep rates slower than $0.00080 \mathrm{MHz} / \mathrm{s}$ can yield accurate DEP response of PS beads as well as RBCs. This sweep rate may therefore be translatable to other cell systems. In addition, at higher frequencies where the polarization mechanism is more heavily influenced by charge permittivity effects through the membrane and cell cytosol, it is possible that slow frequency sweep rates can still accurately capture DEP response spectra. Lastly, this frequency sweep rate 
technique will enable researchers to obtain accurate and continuous DEP response spectra in shorter experiment times.

\subsection{Journal Article References}

1. Adams, T.N.G., K.M. Leonard, and A.R. Minerick, Frequency sweep rate dependence on the dielectrophoretic response of polysytrene beads and red blood cells Biomicrofluidics, 2013. 7: p. 064114.

2. Zhang, C., et al., Dielectrophoresis for manipulation of micro/nano particles in microfluidic systems. Analytical and Bioanalytical Chemistry, 2010. 396(1): p. 401-420.

3. Pethig, R., et al., Dielectrophoresis: A Review of Applications for Stem Cell Research. Journal of Biomedicine and Biotechnology, 2010. 2010: p. 182581.

4. Kim, H.J., et al., Microfluidic device to separate micro-beads with various fluorescence intensities. Sensors and Actuators B-Chemical, 2011. 160(1): p. 1536-1543.

5. Liao, S.H., I.F. Cheng, and H.C. Chang, Precisely sized separation of multiple particles based on the dielectrophoresis gradient in the z-direction. Microfluidics and Nanofluidics, 2012. 12(1-4): p. 201-211.

6. Park, H., M.T. Wei, and H.D. Ou-Yang, Dielectrophoresis force spectroscopy for colloidal clusters. Electrophoresis, 2012. 33(16): p. 24912497. 
7. Holzel, R., Dielectric and dielectrophoretic properties of DNA. Iet Nanobiotechnology, 2009. 3(2): p. 28-45.

8. Grom, F., et al., Accumulation and trapping of hepatitis A virus particles by electrohydrodynamic flow and dielectrophoresis. Electrophoresis, 2006. 27(7): p. 1386-1393.

9. Yasukawa, T., H. Hatanaka, and F. Mizutani, Simple Detection of Surface Antigens on Living Cells by Applying Distinct Cell Positioning with Negative Dielectrophoresis. Analytical Chemistry, 2012. 84(20): p. 8830-8836.

10. Leonard, K.M. and A.R. Minerick, Explorations of ABO-Rh antigen expressions on erythrocyte dielectrophoresis: Changes in cross-over frequency. Electrophoresis, 2011. 32(18): p. 2512-2522.

11. Srivastava, S.K., et al., Dielectrophoretic characterization of erythrocytes: Positive ABO blood types. Electrophoresis, 2008. 29(24): p. 5033-5046.

12. Jen, C.P. and H.H. Chang, A handheld preconcentrator for the rapid collection of cancerous cells using dielectrophoresis generated by circular microelectrodes in stepping electric fields. Biomicrofluidics, 2011. 5(3): p. 10.

13. Salmanzadeh, A., et al., Dielectrophoretic differentiation of mouse ovarian surface epithelial cells, macrophages, and fibroblasts using contactless dielectrophoresis. Biomicrofluidics, 2012. 6(2): p. 13. 
14. Salmanzadeh, A., et al., Isolation of prostate tumor initiating cells (TICs) through their dielectrophoretic signature. Lab on a Chip, 2012. 12(1): p. 182189.

15. Flanagan, L.A., et al., Unique dielectric properties distinguish stem cells and their differentiated progeny. Stem Cells, 2008. 26(3): p. 656-665.

16. Gagnon, Z.R., Cellular dielectrophoresis: Applications to the characterization, manipulation, separation and patterning of cells. Electrophoresis, 2011. 32(18): p. 2466-2487.

17. Xiao, Z.G. and E.F.Y. Young, Placement and Routing for Cross-Referencing Digital Microfluidic Biochips. Ieee Transactions on Computer-Aided Design of Integrated Circuits and Systems, 2011. 30(7): p. 1000-1010.

18. Khoshmanesh, K., et al., Dielectrophoretic platforms for bio-microfluidic systems. Biosensors \& Bioelectronics, 2011. 26(5): p. 1800-1814.

19. Srivastava, S.K., A. Gencoglu, and A.R. Minerick, DC insulator dielectrophoretic applications in microdevice technology: a review. Analytical and Bioanalytical Chemistry, 2011. 399(1): p. 301-321.

20. Cristofanilli, M., et al., Dielectric cell separation of fine needle aspirates from tumor xenografts. Journal of Separation Science, 2008. 31(21): p. 37323739.

21. Wang, X.B., et al., Cell separation by dielectrophoretic field-flowfractionation. Analytical Chemistry, 2000. 72(4): p. 832-839. 
22. Yang, J., et al., Differential analysis of human leukocytes by dielectrophoretic field-flow-fractionation. Biophysical Journal, 2000. 78(5): p. 2680-2689.

23. Markx, G.H. and R. Pethig, Apparatus for separating by dielectrophoresis 1994. p. 17.

24. Lock, G.M., G.H. Markx, and R. Pethig, Manipulation of particles in liquid media. 2001.p. 12.

25. Grosse, C. and A.V. Delgado, Dielectric dispersion in aqueous colloidal systems. Current Opinion in Colloid \& Interface Sciences, 2010. 15: p. $145-$ 159.

26. Morgan, H. and N.G. Green, AC Electrokinetics: colloids and nanoparticles, ed. R. Pethig. 2003, Philadelphia: Research Studies Press Limited.

27. Zhao, H., Double layer polarization of non-conducting particle in an alternating current field with an application to dielectrophoresis. Electrophoresis, 2011. 32: p. 2232-2244.

28. Martinsen, O.G., S. Grimnes, and H.P. Schwan, Interface phenomena and dielectric properties of biological tissue, in Encylcopedia of Surface and Colloid Science. 2002, Marcel Dekker.

29. Pethig, R., Review Article-Dielectrophoresis: Status of the theory, technology, and applications. Biomicrofluidics, 2010. 4(2): p. 35.

30. Mittal, M., et al., Polarization and interactions ac colloidal particles in ac electric fields. Journal of Chemical Physics, 2008. 129: p. 065413. 
31. Gagnon, Z.R. and H.C. Chang, Electrothermal ac electro-osmosis. Applied Physics Letters, 2009. 94: p. 024101.

32. Ghubade, A., et al., Dielectrophoresis assisted concentration of microparticles and their rapid quantitation based on optical means. Biomedical Microdevices, 2009. 11: p. 987-995.

33. Hawkins, B.G., et al., Automated dielectrophoretic characterization of myobacterium smegmatis. Analytical Chemistry, 2011. 83: p. 3507-3515.

34. Chung, C.C., et al., Rapid quantification of bio-particles based on image visualization in a dielectrophoretic microfluidic chip. Microfluidics and Nanofluidics, 2011. 10: p. 311-319.

35. Broche, L.M., et al., Rapid, automated measurement of dielectrophoretic forces using DEP-activated microwells. Electrophoresis, 2011. 32: p. 23932399.

36. Lee, H.J., et al., Rapid and separation-free sandwich immunosensing based on accumulation of microbeads by negative-dielectrophoresis. Biosensors \& Bioelectronics, 2008. 24: p. 1000-1005.

37. Ford, J., Red blood cell morphology. International Journal of Laboratory Hematology, 2013. 35: p. 351-357.

38. Carcao, M.D., The diagnosis and management of congenital hemophilia. Seminars in Thrombosis and Hemostasis, 2012. 38: p. 727-734. 
39. Voskaridou, E., D. Christoulas, and E. Terpos, Sickle cell disease and the heart: review of the current literature British Journal of Haematology, 2012. 157: p. 664-673.

40. Ogata, K., System Dynamics. 1978, Englewood Cliffs: Prentice-Hall Inc.

41. Gascoyne, P., J. Satayavivad, and M. Ruchirawat, Microfluidic approaches to malaria detection. Acta Tropica, 2004. 89: p. 357-369.

\subsection{Chapter Reference}

1. Adams, T.N.G., K.M. Leonard, and A.R. Minerick, Frequency sweep rate dependence on the dielectrophoretic response of polysytrene beads and red blood cells Biomicrofluidics, 2013. 7: p. 064114. 


\title{
Chapter 6 Conclusions: Knowledge Gained from the Dielectrophoretic Study of hMSCs for Type 1 Diabetes
}

\author{
6.1 Summary of Human Mesenchymal Stem Cells, Type 1 Diabetes, and \\ Dielectrophoretic Background Knowledge
}

More than 108,000 children have type 1 diabetes with 16,000 new cases diagnosed each year in the U.S. [1]. There is growing evidence that links gene mutations, viral infections, and bacteria to the autoimmune disruption that causes type 1 diabetes [2-25]. Because the immune system destroys the insulin producing beta cells [26], insulin injections are used as a management tool [27, 28]. No cure exists and the various complications necessitate the need for a curative therapeutic option, which would negate the need for insulin management strategies and reduce the risk of complications from the disease. An effective cure will preserve surviving beta cells, produce new beta cells, and protect all from autoimmune destruction in the pancreas $[29,30]$. hMSCs have been explored as a minimally invasive therapeutic option for beta cell replacement therapy, and there are chemical modifications [31-34], genetic modification $[35,36]$, and nonstimulus methods $[37,38]$ available to differentiate hMSCs toward insulin producing cells.

hMSCs have been explored because they hold high promise as a therapeutic treatment option for type 1 diabetes due to their differentiation ability [39], replenishing capacity [40], and trophic activity [41]. hMSCs are easily obtained from 
bone marrow as a heterogeneous population [42] and require separation and purification before utilization in therapeutics. Within a single hMSC population, there are a variety of progenitor cells (adipogenic-progenitor, osteogenic-progenitor, chondrogenic-progenitor, etc.) and these cells have a proclivity to differentiate toward a specific cell type. Since proclivity is not a $100 \%$ response, purification for individual therapeutic options is important.

Thus far, hMSCs have not been well characterized and there is not a unique catalog of biosurface markers available that distinguishes hMSCs or separate progenitor cells from other cell populations. To compensate, current methodologies utilize density centrifugation paired with either fluorescent-activated cell sorting (FACS) or magnetic-activated cell sorting (MACS) to achieve hMSC subpopulation separations [43]. The main disadvantages for FACS and MACS methods are processing complexity and time, high cost, throughput, and cell antigen labeling; these tedious multistep separation processes are not ideal for clinical scale-up [44]. Therefore, this research explored dielectrophoretic (DEP) microdevices as an alternative. DEP first served as an hMSCs characterization technique and was used to complete the first steps necessary for the design of a microdevice to achieve hMSCs separations for type 1 diabetes therapeutic advancements.

DEP was used in this research because a) surmounting evidence shows that AC electric fields are not harmful to biological cells [45], and b) separations/dielectric signature quantification have been achieved with neural precursor/stem progenitor cells [46], oral squamous cell carcinomas [47], adipose-derived stem cells [48], and 
ovarian cancer cells [49]. Prior research has shown that DEP is a quick, low cost, noninvasive separation method, with non-damaging $\mathrm{AC}$ electric fields [50]. With a custom DEP quadrapole electrode microfluidic device, hMSCs dielectric properties were discerned with membrane capacitance and permittivity measurements. Further, ELP-PEI standardization of hMSC morphology was achieved and dielectric properties were measured within the same DEP device. The specific objectives accomplished in this dissertation research are summarized below.

\subsection{1 hMSC Dielectrophoretic Characterization - Objective 1}

A strong case has been made to use DEP as a characterization tool to determine the dielectric signature for hMSC separation. User-friendly DEP microdevices with low voltage AC electric fields have been demonstrated to rapidly separate cells with no loss in cell viability and virility. The hMSC populations tested in this work were heterogeneous with varied morphology; therefore the development of a continuous cell sorting microdevice should concurrently control and accurately measure hMSCs dielectric signatures independent of cell morphology variations. hMSCs morphology standardization was successfully achieved with ELP-PEI to regulate morphology variations. Additionally, hMSC population heterogeneity correlates to biosurface marker expression predisposing cells for adipocytes, chondrocytes, and osteoblasts. Thus, after morphological standardization, precise measurements of DEP dielectric signatures of individual progenitor cell subpopulations may next facilitate biosurface marker-dependent cell separations. 
hMSCs dielectric dispersion is defined by their internal structure, cell membrane and cytoplasm [50]. Our work has found that hMSCs membrane is recognizable at radio frequencies in the $\beta$-dispersion region. The radio frequencies polarize a cell's surface, facilitating a DEP force, and allowing interogation of the cell membrane dielectric properties [51]. hMSC suspending solution conductivity was found to be critical to cell membrane polarization; higher conductivities increase membrane permittivity shifting the DEP spectra inflection points to higher frequencies. The solution conductivity dependence was apparent at the cross-over frequency, $f_{x o} ; 0.030 \mathrm{~S} / \mathrm{m}$ yields a lower $f_{x o}$ at $0.62 \mathrm{MHz}$ and $0.10 \mathrm{~S} / \mathrm{m}$ yields a lower $f_{x o}$ at $1.3 \mathrm{MHz}$. This dielectric signature is unique for hMSCs because typical low $f_{x o}$ 's for other cell systems fall in the range of $0.010-0.10 \mathrm{MHz}$. Based on these results, these differing DEP spectra could be readily harnessed for hMSC cell separations from other cell systems. It should be noted that untreated hMSC DEP responses at static frequencies varied within a single culture, thus reproducible separations targetting hMSC subpopulations need to consider and control for biosurface marker expression.

This work also explored ELP-PEI treatment, which successfully standardized hMSCs population morphology to spheroids with some cell aggregates observed. This treatment concurrently increased DEP response reproducibility of the ELP-PEI treated hMSCs over the untreated hMSCs. This standardization was facilitated by polymer interaction with the hMSCs cell membrane, which altered membrane dielectric properties and shifted the entire cell's polarization behaviors within the nonuniform AC fields. For ELP-PEI treated cells, only nDEP responses were observed from 
$0.010-10 \mathrm{MHz}$, which was substantially different from native hMSCs. Solution media/membrane polarization behaviors implicate hMSCs are good candidates for future biosurface marker-induced stem cell separations. With optimization of the ELPPEI treatment, detection of molecular level differences in hMSCs may be realizable to aid understanding of biological functions and cell population purification for stem cell therapies.

Therefore, objective 1, as follows, for this disseratioin was successfully accomplished.

Objective 1: Characterize the dielectrophoretic response of hMSCs to determine the DEP spectra including the cross-over frequency.

Objective 1a: Standardize hMSCs morphology with ELP-PEI to reduce size-dependent DEP variations. Characterize the DEP spectra of ELP-PEI treated hMSCs, then compare to native hMSCs in order to identify conditions for cell separations. Subpopulation variations are expected to be based on molecular level expression.

\subsection{2 hMSC Dielectric Properties Modeled - Objective 2}

hMSCs distinct DEP spectra in the $\beta$-dispersion region were correlated to their dielectric properties membrane permittivity and capacity in Chapter 4. Data indicated that higher conductivity solutions increase the membrane permittivity shifting the DEP spectra to higher frequencies. The experimentally determined DEP spectra for hMSCs at $0.030 \mathrm{~S} / \mathrm{m}$ and $0.10 \mathrm{~S} / \mathrm{m}$ were modeled and optimized with the core-shell spherical 
DEP polarization model. The measured conductivity dependence was translated to a membrane capacitance increase from $2.2 \mathrm{pF}$ for $0.030 \mathrm{~S} / \mathrm{m}$ to $4.5 \mathrm{pF}$ for $0.10 \mathrm{~S} / \mathrm{m}$. Similarly, the membrane permittivity increases from 2.0 for $0.030 \mathrm{~S} / \mathrm{m}$ to 4.1 for $0.10 \mathrm{~S} / \mathrm{m}$. Each optimization was a good fit to experimental data. These differing membrane capacitance values are indicative of hMSCs unique dielectric signature; these values are a consistent order of magnitude as values reported within the literature. Thus, these unique dielectric signatures may be harnessed for hMSC cell separations from other cell populations. It should be noted, however, that additional research, discussed in the Future Work section 6.2, should delve into utilizing DEP to discern individual hMSC biosurface markers in order to discern hMSC progenitor cell subpopulations.

Therefore objective 2 for this dissertation research was successfully accomplished.

Objective 2: From the experimentally derived DEP spectra obtained in Objective 1, model the dielectric properties of human mesenchymal stem cells using MATLAB and the Core-Shell Spherical DEP Polarization models. Use these models to calculate the dielectric properties of hMSCs.

Objective 2a: Correlate these properties with known phenotypical molecular expressions of hMSCs.

Objective 2b: Compare dielectric properties with other cell systems, Table 6.1. 
Table 6.1 Cell membrane capacitance comparison between hMSCs and other cell systems. Neural stem/progenitor cells (NSPCs) membrane capacitance was characterized based on its progenitor cells neurogenic progenitor (NP) and astrogenic progenitor (AP) [52]. Human mesenchymal stem cells (hMSCs) membrane capacitance was characterized by work completed in this dissertation [53]. CaLH3, $\mathrm{H} 357, \mathrm{OSCC} 1, \mathrm{DOK}$ are oral squamous cell carcinomas characterized into three subpopulations based on cell adhesion: rapid adherent cells (RAC), middle adherent cells (MAC), and late adherent cells (LAC), which correlates to the cells tumorigenic capabilities [47]. Adipose-derived stem cells membrane capacitance was characterized based on subpopulations determined by differentiation (osteoblasts), and progenitor cells (adipogenic and osteogenic) [48].

\begin{tabular}{|c|c|c|c|}
\hline Cell Type & $\begin{array}{c}\text { Conductivity } \\
(\mathrm{S} / \mathrm{m})\end{array}$ & $\begin{array}{l}C_{\text {mem }} \\
(\mathbf{p F})\end{array}$ & Ref \\
\hline NSPCs-NP biased & 0.01 & 3.3 & {$[52]$} \\
\hline NSPCs-AP biased & 0.01 & 4.3 & {$[52]$} \\
\hline E12.5 mNSPCs & 0.01 & 1.6 & {$[52]$} \\
\hline E16.5 mNSPCs & 0.01 & 2.8 & {$[52]$} \\
\hline $\begin{array}{l}\text { SW Treated-E16.5 } \\
\text { mNSPCs }\end{array}$ & 0.01 & 4 & [52] \\
\hline hMSCs & 0.03 & 2.2 & [53] \\
\hline hMSCs & 0.10 & 4.5 & {$[53]$} \\
\hline treated hMSCs & 0.10 & $<4.5$ & [53] \\
\hline $\begin{array}{ll}\text { CaLH3 } & \text { RAC } \\
& \text { MAC } \\
& \text { LAC }\end{array}$ & 0.10 & $\begin{array}{l}4.1 \\
3.4 \\
2.9\end{array}$ & [47] \\
\hline $\begin{array}{l}\text { RAC } \\
\text { MAC } \\
\text { LAC }\end{array}$ & 0.10 & $\begin{array}{l}3.3 \\
2.9 \\
2.3\end{array}$ & [47] \\
\hline $\begin{array}{l}\text { RAC } \\
\text { MAC } \\
\text { LAC }\end{array}$ & 0.10 & $\begin{array}{l}1.6 \\
1.3 \\
1.1\end{array}$ & [47] \\
\hline RAC & 0.10 & 5.1 & [47] \\
\hline
\end{tabular}




\begin{tabular}{lccc}
\multicolumn{1}{r}{$\begin{array}{l}\text { MAC } \\
\text { LAC }\end{array}$} & & 5.1 & \\
\hline ADSC & $>1.0$ & 5.8 & \\
\hline $\begin{array}{l}\text { Adipogenic- } \\
\text { progenitor }\end{array}$ & $>1.0$ & 9.6 & {$[48]$} \\
\hline $\begin{array}{l}\text { Osteogenic- } \\
\text { progenitor }\end{array}$ & $>1.0$ & 6.9 & {$[48]$} \\
\hline $\begin{array}{l}\text { Differentiated } \\
\text { osteoblasts }\end{array}$ & $>1.0$ & 5.7 & {$[48]$} \\
\hline
\end{tabular}

\subsubsection{Frequency Sweep Rate Dielectrophoretic Experimental Method}

hMSCs dielectric signature quantification in Chapter 4 was achieved using traditional DEP data collection and analysis techniques, which translates to long experimental days and laborious data analysis (months to collect and fully analyze data). A rapid data collection procedure with automated data analysis is beneficial as an alternative technique to quicken the process for DEP characterizations and separations.

Traditional DEP measurements are completed at individual frequencies in order to compile frequency by frequency, the DEP spectrum for a particle or cell system [54]. This method requires time for individual particles to fully polarize and for the dielectrophoretic test region to reach steady state. This can take upwards of $45 \mathrm{~s}$ and is necessary in order to observe accurate DEP responses. An alternative technique is to sweep the frequency or increase frequency via a linear ramp or with small steps over time within a single experiment. This approach allows individual particles to incrementally polarize as the frequency is incrementally increased, thus greatly reducing response time. It was found that frequency sweep rates influence the 
DEP response of polystyrene (PS) beads and red blood cells (RBCs) and further, the permissible frequency sweep rate is particle or cell dependent. At slower sweep rates, particles have more time to polarize in the electric field yielding an accurate and reproducible DEP response and thus quantified spectrum. At faster frequency sweep rates, particles are unable to achieve maximum polarization due to dielectric relaxation of the charges around the particle surface, so the observed DEP response does not match the particle's true DEP behavior.

For PS beads at frequency sweep rates below $0.0063 \mathrm{MHz} / \mathrm{s}$, responses correlate closely with dielectric responses of particles subjected to a static frequency. However, at $0.056 \mathrm{MHz} / \mathrm{s}$, responses began to deviate from static responses, so this was the transitional sweep rate where PS bead dielectric relaxation was approximately the same order of magnitude as the frequency shifts within the sweep. As the sweep rate further increased, conductivity dominated polarizations were not established and the PS bead frequency sweep data did not coincide with static frequency measurements. Similar experiments were completed with red blood cells and results illustrated that $0.00080 \mathrm{MHz} / \mathrm{s}$ accurately predicted the static frequency DEP responses of human RBCs.

Dielectric relaxation is cell specific, so our work demonstrated that for each new cell system it is imperative to determine the optimal frequency sweep rate to accurately and reproducibly interrogate a cell's DEP response. An optimal frequency sweep rate can be estimated by calculating the Maxwell-Wagner dielectric relaxation time for the particle/cell of interest. The applied frequency sweep rate should remain 
at frequencies below the inverse dielectric relaxation time $\left(1 / \tau_{\mathrm{MW}}\right)$ for $\sim 5 \mathrm{~s}$ to achieve slow incremental polarization. This ultimately yields full particle polarization consistent with static frequency polarization measurements. This new frequency sweep rate DEP method will enable researchers to obtain accurate and continuous DEP response spectra in much shorter experiment times and requiring fewer cells.

Therefore, objective 3 of this dissertation work was successfully accomplished. Objective 3: A new DEP data collection technique, frequency sweep rate, was established for rapid compilation cell's DEP spectrum. An optimization procedure was identified for the operational frequency sweep rate for effective characterization of specific cells DEP spectrum. The method was successfully verified with polystyrene beads and red blood cells. Additional semi-automated data analysis was tested on polystyrene beads and red blood cells; a patent disclosure has been filed on this work, with the full patent application to be submitted in September 2014.

In summary, dielectrophoretic microdevice versatility has been demonstrated within this dissertation research through hMSC quantification, hMSC treatment, and the development of a new DEP data collection and quantification technique. New knowledge has been contributed to the field including hMSCs DEP spectra, membrane capacitance, permittivity, interactions with ELP-PEI, and the development of the new frequency sweep rate DEP data collection/analysis technique. 


\subsection{Future Work}

The first steps toward the development of a continuous cell sorting DEP microdevice was completed in this dissertation through the quantification of a heterogeneous hMSCs population's dielectric signature and the implementation of an ELP-PEI morphological standardization technique. The next generation hMSC microdevice should include an optimized DEP sorting chamber, which bifurcates into additional microchannels and outlet collection ports. Such a design could be optimized to allow continuous flow for hMSCs separation, thus moving the current system from a batch to a continuous process. This work is achievable by the utilization of the microfabrication facility in the M\&M building on campus, and would allow a tailor made device to be developed for hMSCs.

Further investigations should be completed, with the new continuous cell sorting DEP microdevice, to achieve hMSCs separations based their progenitor cell phenotype. This is crucial in designing a device specifically for clinical therapeutics, and allows cell fate prediction.

Another parameter that should be further explored is the ELP-PEI treatment. It should be noted that only one concentration of ELP-PEI was tested. The concentration was based upon the optimal concentration required for hepatocyte cells [55]. However, the hMSC cells displayed not only spheroidal shape, but also a tendency to form into spheroid aggregates of multiple cells. To avoid these cell-cell interactions, concentration optimization studies should be completed such that the minimal concentration of ELP-PEI is present in the system to adjust individual cell 
morphologies. Additionally, further studies should be completed to assess the impact the ELP-PEI treatment has on hMSCs differentiation capacity and trophic activity.

Although, further studies with hMSCs and ELP-PEI should be completed to fully understand molecular level interactions together, it is important to mention that utilizing this treatment will have a positive impact on hMSCs separation because of the substantial shift in cross over frequency and DEP spectra. As a result of the treatment, the $\mathrm{nDEP}$ behavior observed over a wide frequency range in the future will allow separation from other cell systems. Dissolution studies and time necessary for the ELP-PEI coating to be removed from hMSCs, should to be explored in order to design a washing step on the microfluidic device. This step will allow ELP-PEI to be removed before purified hMSCs are cultured and used in type 1 diabetes therapy.

Also within this body of work, the DEP data collection and analysis technique was further improved using a frequency sweep rate method, which allows multiple frequencies, be examined within one experimental test. This method is currently semiautomated using data analysis with ImageJ. For further improvement toward fully automated data collection, MATLAB code should be instituted to automatically analyze data collected with the microdevice. This rapid, fully automated system is advantageous for separations of both hMSC from other cell types and hMSC progenitor cell subpopulations. This is necessary to make this approach competitive with FACS and MACS. Further, dielectric signatures may vary slightly from donor to donor, so being able to rapidly quantify donor specific hMSC membrane capacitance and then in situ adapt operating conditions would provide the versatility to 
concurrently separate hMSCs from other cells in heterogeneous populations. Thus, dielectrophoretic separations have the potential to serve as a powerful separation tool that would enable large-scale, more cost-effective type 1 diabetes autologous therapy.

\subsection{References}

1. Federation, I.D. IDF diabetes atlas. 2013 [cited 2014 March 25]; 6:[Available from: http://www.idf.org/diabetesatlas.

2. Bacchetta, R., et al., Defective regulatory and effector $T$ cell functions in patients with FOXP3 mutations. Journal of Clincal Investigation, 2006. 116(6): p. $1713-1722$.

3. Bell, G.I., S. Hortia, and J.H. Karam, A polymorphic locus near the human insuline gene is associated with insulin-dependent diabetes-mellitus. Diabetes, 1984. 33(2): p. 176-183.

4. Bennett, S.T., et al., Susceptibility to human type-1 diabetes at IDDM2 is determined by tandem repeat variation at the insulin gene minisatellite locus. Nature Genetics, 1995. 9(3): p. 284-292.

5. Bottini, N., et al., A functional variant of lymphoid tyrosine phosphastase is associated with type 1 diabetes. Nature Genetics, 2004. 36(4): p. 337-338.

6. Bussone, G. and L. Mouthon, Autoimmune manifestations in primary immune deficiencies. Autoimmunity Reviews, 2009. 8(4): p. 332-336.

7. Clements, G.B., D.N. Galbraith, and K.W. Taylor, Coxsackie-B virus-infection and onset of childhood diabetes. Lancet, 1995. 346(8969): p. 221-223. 
8. Craig, M., et al., Viruses and type 1 diabetes: a new look at an old story. Pediatric Diabetes, 2013. 14(3): p. 149-158.

9. Dai, Y.D., et al., Slcllal enhances the autoimmune diabetogenic T-cell response by altering processing and presentation of pancreatic islet antigens. Diabetes, 2009. 58(1): p. 156-164.

10. Dippe, S.E., et al., Lack of causal association between coxsackie-B4 virusinfection and diabetes. Lancet, 1975. 1(7920): p. 1314-1317.

11. Gamble, D.R., et al., Viral antibodies in diabetes mellitus. British Medical Journal, 1969. 3(5671): p. 627-630.

12. Guberski, D.L., et al., Induction of type-1 diabetes by kilham's rat virus in diabetes-resistant BB/WOR rats. Science, 1991. 254(5034): p. 1010-1013.

13. Lonnrot, M., et al., Enterovirus infection as a risk factor for beta-cell autoimmunity in a prospectively observed birth cohort- the finnish diabetes prediction and prevention study Diabetes, 2000. 49(8): p. 1314-1318.

14. Lonnrot, M., et al., Enterovirus RNA in serum is a risk factor for beta-cell autoimmunity and clincal type 1 diabetes: a prospective study Journal of Medical Virology, 2000. 61(2): p. 214-220.

15. Lowe, C.E., et al., Large-scale genetic fine mapping and genotype-phenotype associations implicate polymorphism in the IL2RA region in type 1 diabetes. Nature Genetics, 2007. 39(9): p. 1074-1082.

16. Pak, C.Y., et al., Association of cytomegalo-virus infection with autoimmune type-1 diabetes. Lancet, 1988. 2(8601): p. 1-4. 
17. Ueda, H., et al., Associaton of the T-cell regulatory gene CTLA4 with susceptibility to autoimmune disease Nature 2003. 423(6939): p. 506-511.

18. Undlien, D.E., et al., HLA-encoded genetic predisposition in IDDM - DR4 subtypes may be associated with different degrees of protection. Diabetes, 1997. 46(1): p. 143-149.

19. Vaarala, O., M.A. Atkinson, and J. Neu, The "perfect storm" for type 1 diabetes - the complex interplay between intestinal microbiota, gut permeability, and mucosal immunity. Diabetes, 2008. 57(10): p. 2555-2562.

20. Van Belle, T.L., K.T. Coppieters, and M.G. Von Herrath, Type 1 diabetes: etiology, immunology, and therapeutic strategies. Physiological Reviews, 2011. 91(1): p. 79-118.

21. Villasenor, J., C. Benoist, and D. Mathis, AIRE and APECED: molecular insights into an autoimmune disease Immunological Reviews, 2005. 204: p. $156-164$.

22. Wildin, R.S., et al., X-linked neonatal diabetes mellitus, enteropathy, and endocrinopathy syndrome is the human equivalent of mouse scurfy. Nature Genetics, 2001. 27(1): p. 18-20.

23. Yoon, J.W., et al., Virus-induced diabetes-mellitus - isolation of a virus from the pancreas of a child with diabetic ketoacidosis. New England Journal of Medicine, 1979. 300(21): p. 1173-1179.

24. Yoon, J.W., T. Onodera, and A.L. Notkins, Virus-induced diabetes-mellitus. $X V$. beta cell damage and insulin-dependent hyperglycemia in mice infected 
with coxsaxkie virus-B4. Journal of Experimental Medicine, 1978. 148(4): p. 1068-1080.

25. Zanone, M.M., et al., Association of cytomegalovirus infections with recurrence of humoral and cellular autoimmunity to islet autoantigens and of type 1 diabetes in a pancreas transplanted patient. Transplant International, 2010. 23(3): p. 333-337.

26. Galadari, S., et al., Role of ceramide in diabetes mellitus: evidence and mechanisms. Lipids in Health and Disease, 2013. 12: p. 98.

27. Jang, Y.K., et al., Optimization of the therapeutic efficacy of human umbilical cord blood-mesenchymal stromal cells in an NSG mouse xenograft model of graft-versus-host disease. Cytotherapy, 2014. 16: p. 298-308.

28. Malik, F.S. and C.E. Taplin, Insulin therapy in children and adolescents with type 1 diabetes. Pediatric Drugs, 2014. 16(2): p. 141-150.

29. Aguayo-Mazzucato, C. and S. Bonner-Weir, Stem cell therapy for type 1 diabetes mellitus. Nature Reviews Endocrinology, 2010. 6(3): p. 139-148.

30. Zheng, G.P., et al., Mesenchymal stem cells in the treatment of pediatric diseases. World Journal Pediatrics, 2013. 9: p. 197-211.

31. Fiorina, P., J. Voltarelli, and N. Zavazava, Immunological applications of stem cells in type 1 diabetes. Endocrine Reviews, 2011. 32(6): p. 725-754.

32. Sun, Y., et al., Differentiation of bone marrow-derived mesenchymal stem cells from diabetic patients into insulin-producing cells in vitro. Chinese Medical Journal, 2007. 120(9): p. 771-776. 
33. Volarevic, V., et al., Concise review: mesenchymal stem cell treatment of the complications of diabetes mellitus. Stem Cells 2011. 29(1): p. 5-10.

34. Xie, Q.P., et al., Human bone marrow mesenchymal stem cells differentiate into insulin-producing cells upon microenvironmental manipulation in vitro. Differentiation, 2009. 77(5): p. 483-491.

35. Karnieli, O., et al., Generation of insulin-producing cells from human bone marrow mesenchymal stem cells by genetic manipulation. Stem Cells, 2007. 25(1): p. 2837-2844.

36. Li, Y.H., et al., Generation of insulin-producing cells from PDX-1 gene modified human mesenchymal stem cells Journal of Cellular Physiology, 2007. 211(1): p. 36-44.

37. Lee, R.H., et al., Multipotent stromal cells from human marrow home to and promote repair of pancreatic islets and renal glomeruli in diabetic NOD/scid mice. PNAS, 2006. 103(103): p. 17438-17443.

38. Leeb, C., et al., New perspectives in stem cell research: beyond embryonic stem cells Cell Proliferation, 2011. 44: p. 9-14.

39. Salem, H.K. and C. Thiemermann, Mesenchymal stromal cells: current understanding and clinical status. Stem Cells, 2010. 28: p. 585-596.

40. He, S., D. Nakada, and S.J. Morrison, Mechanisms of stem cell self-renewal. Annual Review of Cell and Development Biology, 2009. 25: p. 377-406.

41. Caplan, A. and J.E. Dennis, Mesenchymal stem cells as trophic mediators. Journal of Cellular Biochemistry, 2006. 98: p. 1076-1084. 
42. Wu, H.W., C.C. Lin, and G.B. Lee, Stem cells in microfluidics. Biomicrofluidics, 2011. 5: p. 013401.

43. Diogo, M.M., C. Lobato de Silva, and J.M.S. Cabral, Separation technologies for stem cell bioprocessing. Biotechnology and Bioengineering, 2012. 109: p. 2699-2709.

44. Gonzalez-Gonzalez, M., et al., Current strategies and challenges for the purification of stem cells. Journal of Chemical Technology and Biotechnology, 2012. 87: p. 2-10.

45. Lu, J., et al., Advancing practical usage of microtechnology: a study of the functional consequences of dielectrophoresis on neural stem cells. Integrative Biology, 2012. 4: p. 1223-1236.

46. Flanagan, L.A., et al., Unique dielectric properties distinguish stem cells and their differentiated progeny. Stem Cells, 2008. 26: p. 656-665.

47. Liang, X., et al., Human oral cancer cells with increasing tumorigenic abilities exhibit higher effective membrane capacitance. Integrative Biology, 2014. 6: p. $545-554$.

48. Bagnaninchi, P.O. and N. Drummond, Real-time label-free monitoring of adipose-derived stem cell differentiation with electric cell-substrated impedance sensing. PNAS, 2011. 108: p. 6462-6467.

49. Salmanzadeh, A., et al., Sphingolipid metabolites modulate dielectric characteristics of cells in a mouse ovarian cancer progression model. Integrative Biology, 2013. 5: p. 843-852. 
50. Pethig, R., Review Article-Dielectrophoresis: Status of the theory, technology, and applications. Biomicrofluidics, 2010. 4(2): p. 35.

51. Adams, T.N.G., K.M. Leonard, and A.R. Minerick, Frequency sweep rate dependence on the dielectrophoretic response of polysytrene beads and red blood cells Biomicrofluidics, 2013. 7: p. 064114.

52. Nourse, J.L., et al., Membrane biophysics define neuron and astrocyte progenitors in the neural lineage. Stem Cells, 2014. 32: p. 706-716.

53. Adams, T.N.G., et al., Characterizing the dielectric properties of human mesenchymal stem cells and the effects of charged elastin-like polypeptide copolymer treatment Biomicrofluidics, 2014. submitted.

54. Gagnon, Z.R., Cellular dielectrophoresis: Applications to the characterization, manipulation, separation and patterning of cells. Electrophoresis, 2011. 32(18): p. 2466-2487.

55. Janorkar, A.V., et al., Use of three-dimensional spheroids of hepatocytederived reporter cells to study the effects of intracellular fat accumulation and subsequent cytokine exposure. Biotechnology and Bioengineering, 2011. 108: p. 1171-1180. 


\section{Appendix A: A Tunable Microfluidic Device for Drug}

\section{Delivery}

${ }^{1}$ Tayloria Adams, Chungja Yang, John Gress, Nick Wimmer, Adrienne R. Minerick Michigan Technological University

USA

\section{A.1 Introduction}

The field of microfluidics, small-scale tests from nanoscale to microscale, has grown dramatically over the past two decades as evidenced by greater than 30,000 papers published over the last 10 years on the topic [Web of Knowledge search using 'microfluidic' terms October 2011]. Microfluidic platforms, also known as lab-on-achip (LOC), include a set of miniaturized integrated unit operations that are touted to lead to fast, easy, precise control in biological and chemical systems. LOCs include the development of point-of-care (POC) medical diagnostic devices with the advantages of increased sensitivity, lower sample volumes, lower reagent volumes, low energy, low cost, low labor need, and less likelihood of human error (Xiao \& Young, 2011). Due to these advantages, LOCs have substantial potential to be widely utilized in medicine for analytical and diagnostic assays, biosensors, and drug delivery.

\footnotetext{
${ }^{1}$ The material contained in this Appendix was previously published in Advances in Microfluidics, 2012.
} 
Microfluidic technology has been used for a wide variety of applications such as forensics, cell phone facilitated micro-imaging, and analytical testing. In 2006 Bienvenue at al., compared the use of microfluidic technology with a commercial kit that utilized dithiothreitol to extract and purify DNA from sperm samples. The sample volume was less than $10 \mu \mathrm{L}$ and the resulting electropherograms were very similar for both techniques (Bienvenue et al., 2006). DNA separation has also been studied by Aboud et al. Pentameric short tandem repeat (STR) markers were tested in a microfluidic device on single-stranded DNA. Coupling microfluidics with pentameric STRs improved allele resolution by 3.7 times (Aboud et al., 2010). In these cases, microfluidics can be used as a rapid screening tool for forensic DNA analysis to help resolve the backlog of DNA casework (Aboud et al., 2010; Bienvenue et al., 2006). Zhu et al., combined optofluidics with cell phone technology. A cell phone was converted to a microscope analysis tool by integrating optofluidic fluorescent cytometry with compact optical attachments. The cell phone optical attachment included a lens, plastic color filter, two light emitting diodes, and batteries, which altogether weighed less than $1 \mathrm{lb}$. To test the effectiveness of this new imaging system, the density of white blood cells were measured using the cell phone-based fluorescent image cytometry and compared with the white blood cell density found with a commercial hematology analyzer. The blood sample was injected into the microfluidic chamber using a syringe pump and the cell phone recorded the fluorescent emission. This study demonstrated that the densities found by both systems were a good match with less than $5 \%$ error and that cell phone optofluidic fluorescent imaging cytometry 
was useful for rapid blood cell counts or screening of water quality (Zhu et al., 2011).

Research into microfluidic devices tailored for the medical field is extensive. Weng et al., developed a suction type microfluidic device to detect the dengue virus. This three-layer device used pneumatics, mixing, and transport to detect the virus in 30 minutes (Weng et al., 2011). Digital microfluidic devices transport biochemical materials in the form of miniature discrete droplets (Xiao \& Young, 2011) and have been used for immunosensing, proteomics, DNA, and cell based assays (Vergauwe et al., 2011). Dielectrophoresis (DEP) has been incorporated into microfluidic devices for transport, separation, and blood typing (Minerick et al., 2008; Srivastava et al., 2011; C. Wang et al., 2011). DEP phenomena is the movement of cells from an external applied electric field and has been used to continuously separate breast cancer cells from normal blood cells (Alazzam et al., 2011). The device developed by Alazzam et al. can potentially be used as an early detection method for cancer.

Professor Robert Langer and other researchers at MIT investigated the idea of a "pharmacy on a chip". They performed controlled release studies to determine if a microfluidic platform could act as a pulsatile release drug delivery system. Pulsatile release is a common controlled release method used to treat people with disorders that require drugs to be delivered at varying rates over time. A prototype microchip made from silicon was developed. The microchip had multiple reservoirs for drug storage and the reservoirs were covered with gold membranes. The reservoirs were filled with sodium fluorescein and calcium chloride using ink jet printing. To release the drugs an electric potential of approximately $1 \mathrm{~V}$ was applied and the gold membranes were 
dissolved in 10 to 20 seconds. The results from this study revealed that storage and on-demand delivery of drugs can be achieved from microfluidic LOC technology. One major advantage of using microfluidic platforms for drug delivery is that small microchips can be implanted inside the body to locally treat diseases (Santini et al., 1999, 2000). Farokhzad et al. gave a possible application of microfluidic technology in the field of urology (Farokhzad et al., 2006). Other researchers have implemented the proof-of-concept that Langer demonstrated for ambulatory emergency care treatment. A plethora of drug delivery systems that can be embedded in the body have been researched for use in chronic and non-chronic diseases. When treating chronic and non-chronic diseases drugs are delivered over long periods of time. These systems are now modified to rapidly deliver drugs in emergency situations (Elman et al., 2009). Elman et al. developed a smart microchip implant to deliver a drug bolus when disease symptoms are detected. The device is composed of three layers: reservoir layer where drug solution is stored, membrane layer where reservoir is sealed and location of drug is released, and actuation layer where bubbles are formed to trigger the release of the stored drugs. The actuation layer triggers the operation of the device. Micro-resistors heat the drug to generate bubbles, pressure is produced, and the membranes burst delivering the drug $(\approx 20 \mu \mathrm{L})$ rapidly from the device to its target area in 45 seconds. In this work vasopressin was used as the drug and it was found that $92.5 \%$ of the solution loaded into the device was released. Devices of this nature have the potential to accompany cardiac devices such as defibrillators and pacemakers (Elman et al., 2009). Langer's findings have even been extended to nanotechnology. Brammer et al., 
has shown that silicon nanowires are a viable drug delivery system for antibiotics. It was shown that silicon nanowires sustained drug release levels for 42 days (Brammer et al., 2009).

Despite this wide breadth of research success, commercial implementation of POC devices for diagnostics assays, biosensors, and drug delivery have been much slower than originally predicted. A feature article in Time magazine in 2001 exemplified this dream touting safer and more effective drug delivery techniques (Bjerklie \& Jaroff, 2001). However, only a few notable LOC platforms have come to market and are most advanced in the areas of bioassays (pregnancy/ovulation tests, etc.) and gene chips. Bioassay companies include eBioscience, and Chembio Diagnostic Systems, Inc. Notable gene profiling chips include those by Affymetrix, Fluidigm, Gyros, and Sage. Blood chemical analyzers are marketed by PiccoloXpress, while versatile analytical LOCs are marketed by Caliper and Dolomite Microfluidics. Commercialization is more advanced in the diagnostics arena than in the drug delivery area due to the complexity of sensing the concentration of the drug and controlling the release of new drug. However, as demonstrated by the growth in foundational research, popular news source stories, and commercialization of products, new innovations in this area are being sought.

Cancer is a disease that touches everyone in the world; people are either directly affected by cancer or know someone suffering from the disease. Globally, cancer is responsible for $1 / 8^{\text {th }}$ of all deaths, which is more than HIV/AIDS, malaria, and tuberculosis combined (American Cancer Society, 2011). It is estimated that 1.5 
million new cases of cancer will be diagnosed in the U.S. in 2011. Cancer is growing at an increasingly high rate and it is expected that there will be 21.4 million new cases of cancer in 2030 and 13.2 million cancer deaths (American Cancer Society, 2011). Gastric cancer is malignant cell growth originating in the gastro-intestinal tissue lining and kills 650,000 people with 870,000 new cases diagnosed annually (BalcerKubiczek \& Garofalo, 2009). It is the second most fatal disease in the world (BalcerKubiczek \& Garofalo, 2009; National Cancer Institute, 2010) and has a poor prognosis due in part to late stage development of any symptoms. People diagnosed with gastric cancer often do not experience symptoms until the disease is metastatic and spreading elsewhere in the body. This then dictates systemic chemotherapy treatment, which traditionally is conducted with regular injections or an embedded catheter. These methods add suffering and additional pain beyond the discomforts of chemotherapy. Further, these methods of drug delivery have large variations in patient exposure concentrations over the course of the treatment; survival rates for gastric cancer suggest this approach is not entirely effective. Therefore, there is a great need for development of new technology to treat cancer patients. The new technology should have two goals (1) effectively treat cancer patients to eradicate disease and (2) make cancer treatments as painless and noninvasive as possible. Here we wish to combine four unique technologies into a microfluidic device to provide novel nanoscale drug delivery for cancer patients via a wrist device resembling a watch. Figure A.1 shows the global view of our chemotherapy drug delivery system and Figure A.2 shows the how these four technologies fit together on the drug-delivery microfluidic device and 
are then discussed separately in the following sections.

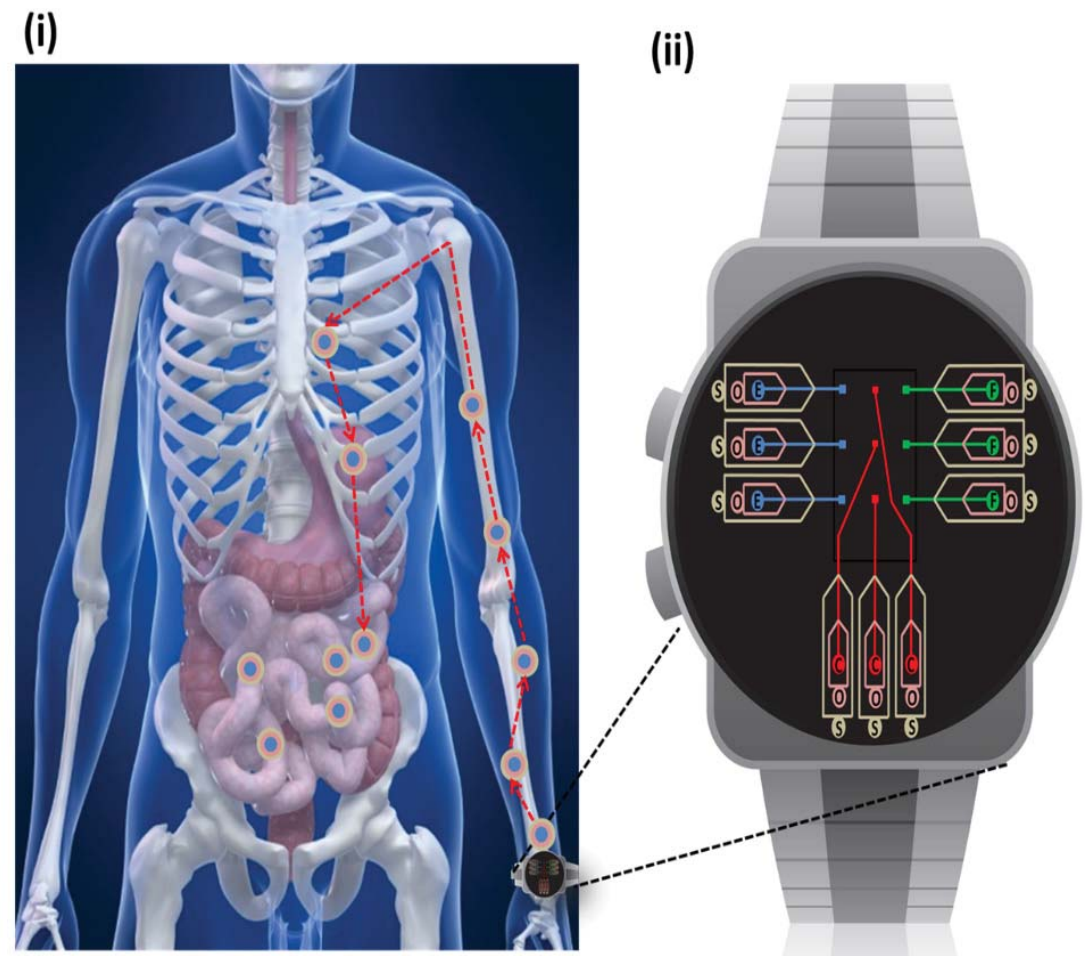

Figure A.1 Global view of chemotherapy drug delivery system, (i) path of emulsified drug from the wrist microdevice through the human body and (ii) enlarged view wrist devices depicting the chemotherapy drug delivery system. The encapsulated chemotherapy drug droplets travel from the wrist device to the intestines contacting circulating tumor cells (CTCs) to treat the gastric cancer.

In Figure A.2(i), the reservoirs for each drug are centralized into larger chambers above the layers shown in Figure A.2(iv). There is a primary and three 
secondary reservoirs for oil (one for each drug), and the same for saline. The primary reservoir allows the flow rate of each drug to be independently controlled. This device uses microchannels and tunable electrodispersion to form in-line emulsions of the chemotherapy drug, which are then delivered to the patient using adjustable dielectrophoretic pumping and painless microneedles that penetrate the dermis of the skin. The focus of this new technology has been to specifically treat gastric cancer, but can be adapted to treat many other types of cancer and possibly other diseases.
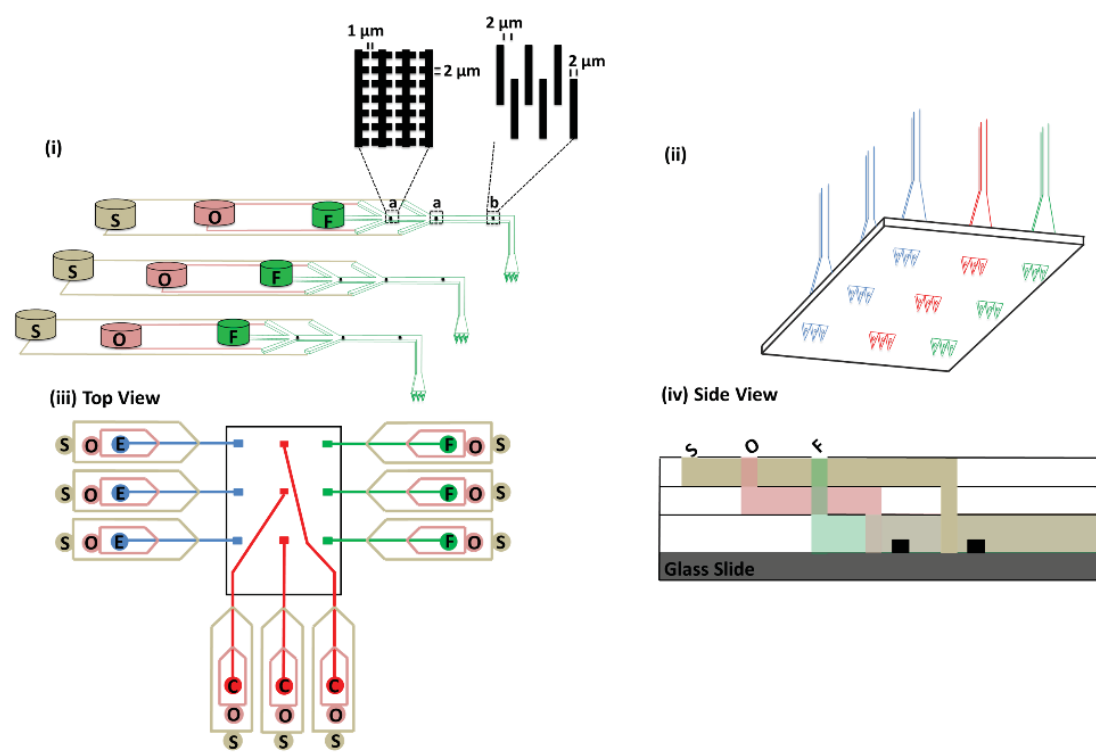

(iv) Side View

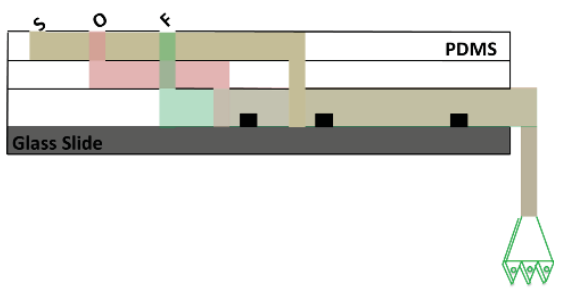

Figure A.2 Microfluidic drug delivery device for cancer treatment. (i) Overview of fluorouracil drug system including drug, oil and saline storage including (a) electrodispersion electrodes and (b) dielectrophoretic micropumping electrodes. Microchannel dimensions are $25 \mu \mathrm{m}$ (width) x $25 \mu \mathrm{m}$ (height). (ii) Termination of microchannels into the microneedle system. (iii) Top view of ECF droplet microdevice. And (iv) Side view of multilayered system for droplet dispersion and 
flow to the microneedles. The abbreviations are $\mathrm{S}=$ saline, $\mathrm{O}=$ poppy seed oil, $\mathrm{F}=$ fluorouracil, $\mathrm{E}=$ epirubicin, and $\mathrm{C}=$ cisplatin.

Chemotherapy is a common treatment option for gastric cancer. Several single chemotherapy drugs have been used to treat gastric cancer including 5-fluorouracil, mitomycin, doxorubicin, cisplatin, etoposide, docetexal, and methotrexate. Efficacy of these drugs are typically measured via clinical response rates, which is the percentage of patients that respond to cancer treatment such that cancer cells are no longer detected. The response rates to these drugs were poor ranging from $15-35 \%$ (Cleveland Clinic Foundation, 2010; Hershock, 2006; Levi et al., 1979). More effective treatments use a combination of two, three or more chemotherapy drugs. Combining two chemotherapy drugs has been examined by Levi et al. and response rates for drug cocktails increased to 40-50\% (Levi et al., 1979). McDonald et al. combined three chemotherapy drugs fluorouracil, doxorubicin, and mitomycin and results showed a 55\% response rate (Levi et al., 1979). Rivera et al. studied docetexal, a newer chemotherapy drug, in combination with cisplatin and 5-fluorouracil (DCF). DCF was compared with docetexal/cisplatin (DC) and cisplatin/5-fluorouracil (CF), and the objective response rates for DCF were $37-43 \%, 26 \%$ for DC, and $25 \%$ for $\mathrm{CF}$. Based on these results it can be concluded that a combination of three chemotherapy drugs are more effective than two chemotherapy drugs (Rivera et al., 2007). Other combination chemotherapy drugs have been studied, and their response rates were: epirubicin, cisplatin and, 5-fluorouracil (ECF) 71\%; 5-fluorouracil, adriamycin, and 
mitomycin 50\% and 9\%; 5-fluorouracil, leucovorin, and cisplatin 44\%; 5-fluorouracil, adriamycin, mitomycin, and methotrexate $42 \%$; cisplatin, epirubicin, leucovorin, and 5-fluorouracil 43\% docetexal, cisplatin, and 5-fluorouracil 37-43\% (Hershock, 2006; Power et al., 2010). Epirubicin, cisplatin, and 5-fluorouracil (ECF) had the highest response rate of $71 \%$. In summary, response rate data suggests that combination chemotherapy drug treatment is the superior treatment option. Therefore, the microfluidic device described here will utilize the combination of ECF. In the device depicted in Figures A.1 and A.2 each individual drug in the ECF drug system is stored in separate reservoirs so their dosage can be independently controlled via feedback electronics. There is also redundancy in the microchannels and microneedles for backup in case any of the channels become clogged over time. Within the microchannels each drug is sheathed in a biocompatible oil in order to protect the integrity and enhance drug efficacy over the dosage cycle.

This chapter will further explore the integration of microchannels, electrodispersion, dielectrophoretic pumping, and microneedles in a dynamically controllable microfluidic platform to deliver ECF to gastric cancer patients.

\section{A.2 Technologies Utilized in the Drug Delivery Microfluidic Device}

\section{A.2.1 Microchannels and Electrodispersion}

Emulsions are mixed dispersions of more than two immiscible fluids via encapsulation of one layer by the other layer. These emulsion droplets are useful in areas such as foods, cosmetics, pharmaceutical drug delivery, and chemical synthesis. 
Examples of foods include milk, yogurt, sauce, butter, etc. and cosmetics of lotion (oilin-water, $\mathrm{O} / \mathrm{W}$ ), cream (water-in-oil, W/O), hair, shaving, and bath products that are predominantly viscous liquids (Mezzenga, 2005; S.H. Kim et al., 2011). In chemical synthesis, droplets are being used as a new reaction platform due to their ability to function as a batch reactor such as antimicrobial agent and preservatives (Hamouda et al., 1999; Jensen \& Lee, 2004; Mejia et al., 2009). The forms of emulsion droplets to contain various physical and chemical compositions are effective in delivering drugs and cosmetics in human body (Wibowo \& Ng, 2001; Kiss et al., 2011).

In this drug delivery microfluidic device, flow focusing $(\mathrm{FF})$ hydrodynamics and electrodispersion technology are combined to dynamically generate oil-sheathed drug droplets on the order of $100 \mathrm{~nm}$ outer diameter dispersed in saline. Poppy seed oil is used to decrease the toxicity of the chemotherapy drug (Pai et al., 2003) while maintaining its potency and efficacy once it reaches the target malignant cells, and saline is used to carry the droplets into the tissue during injection. Our drug delivery microdevice will combine both flow focusing and electrodispersion technologies in order to achieve narrower size distribution of particles by preventing droplet interactions and coalescence. Electric fields are added for chemotherapy drug droplet formation to decrease the size of droplets, improve robustness of continuity of the droplet thread formed, and increase velocity as droplets travel downstream in the microchannel. Thus, the main focus of our study is developing FF geometry with electrodispersion and adequate use of surfactants to generate submicron droplets $(\sim 100$ $\mathrm{nm}$ ) with highly uniform sizes to promote a quick transport into cells. 
Emulsion droplets of very narrow size distributions can be strategically generated by harnessing hydrodynamic behaviors within microfluidic systems (Anna et al., 2003; Martin-Banderas et al., 2005; W. Lee et al., 2009). FF geometries are used in mixing immiscible phases or encapsulating one phase within a second sheathing phase. Typically, hydrophobic drops are dispersed in a hydrophilic fluid or vice versa. The drug delivery microdevice utilizes this technology in order to protect the inner fluid (drug) by an outer fluid (oil), which is then dispersed in a continuous saline stream. The inner phase is traditionally termed the dispersed phase while the outer phase is termed the continuous phase when they meet at an orifice. Most microfluidic emulsions involve a single droplet dispersed in a continuous phase such as water-inoil (W/O) and oil-in-water (O/W) (Ha and Yang, 1999; Anna et al., 2003; W. Lee et al., 2009; Kiss et al., 2011). This concept can also be expanded to droplets that include more than one internal droplet such as double emulsions $(\mathrm{W} / \mathrm{O} / \mathrm{W}$ or $\mathrm{O} / \mathrm{W} / \mathrm{O})$ (Utada et al., 2005; Seo et al; 2007; Liao and Su, 2010; S.H. Kim et al., 2011) and are the foundation for the drug delivery microdevice described in this chapter.

During small droplet synthesis, mechanical shear stress was utilized in order to achieve small and highly stable emulsion droplets, but this approach yielded tens of nano- to hundreds of micro-scale droplets with large size distributions, which was problematic (Pacek et al., 1999; Abismail et al., 1999). The key advantage of FF technique is precise control in producing droplets into the range of hundreds of nanometers (Anna et al., 2003; Thiele et al., 2010), but ambiguity remains regarding the lower limit of droplet sizes that can be achieved, the size distribution and continuity 
of the droplet threads still remain unreported due in part to the difficulty of in-line droplet size analysis and the length of the droplet thread (Anna et al., 2003; W. Lee et al., 2009). The goal in this drug delivery microdevice is to generate the smallest droplets possible because larger droplets are less stable and more likely to come in contact with each other, which leads to droplet deformation and coalescence. Other studies have determined that 50-150 $\mathrm{nm}$ droplets ensure an optimal intake in cells for drug delivery applications (Thiele et al., 2010). In addition, larger droplets are less stable and more likely to come in contact with each other which leads to droplet deformation and coalescence.

Because the drug delivery microfluidic system dimensions are designed to fit within a wristwatch-like system on a human wrist, multilayered FF and electrodispersion is proposed for ECF chemotherapy drug emulsion and delivery, Figure A.2(ii-iv). The total dimensions of multilayered ECF drug system is small enough to be non-obtrusive, so that it can be worn for continuous drug delivery with minimal discomfort as shown in Figure A.1. In the drug delivery microdevice, two FF orifice/junction geometries are utilized in series as shown in Figure A.3. The two junctions whereby sheathing flows of poppy seed oil and saline are added to the main channel are $3 \mathrm{~mm}$ long, $20 \mu \mathrm{m}$ wide, and $20 \mu \mathrm{m}$ deep in the microchannel, and the continuous and dispersed phases are injected through pressure regulated membrane deflection into the fluid reservoirs which operate as micropumps. Such designs are also commercially available and meet the volume and portability limitations of the proposed wristwatch system, as well as the energy demand limitations (Lima et al., 
2004). The flow rate inputs are $0.01 \mu \mathrm{l} / \mathrm{min}$ of drug, $0.1 \mu \mathrm{l} / \mathrm{min}$ for poppy seed oil, and $1 \mu 1 /$ min for saline based on the reduction of the optimal flow conditions achieved by Zagnoni et al., 2009 \& 2010 to meet our channel dimensions in which the ratio of the continuous and dispersed phase flow rate are held at 10 to achieve submicron droplets in the downstream microchannels.

Achieving drug droplets with diameters at approximately $500 \mathrm{~nm}$ is feasible via the hydrodynamic flow focusing achieved with the $4 \mu \mathrm{m}$ wide orifice and combined with the strategic use of surfactant chemistry (W. Lee et al., 2009). Droplet size is determined initially by the orifice geometry; however, a surfactant mixed with either the continuous or dispersed phase balances interfacial tension and enables droplet sizes to be orders of magnitude smaller in comparison with those without a surfactant (W. Lee et al., 2009). That is, adding a proper surfactant can improve stability of the droplets and decrease the size of droplets because the surfactant molecules reduce the interfacial tension between different fluids in a droplet, thus avoiding the undesirable coalescence among droplets. For this chemotherapy drug delivery application, proper surfactant selection requires biocompatibility, which must be considered as well as long-term drug-surfactant interactions. To avoid the later, surfactants will be dispersed in the oil phase and in the saline phase. 


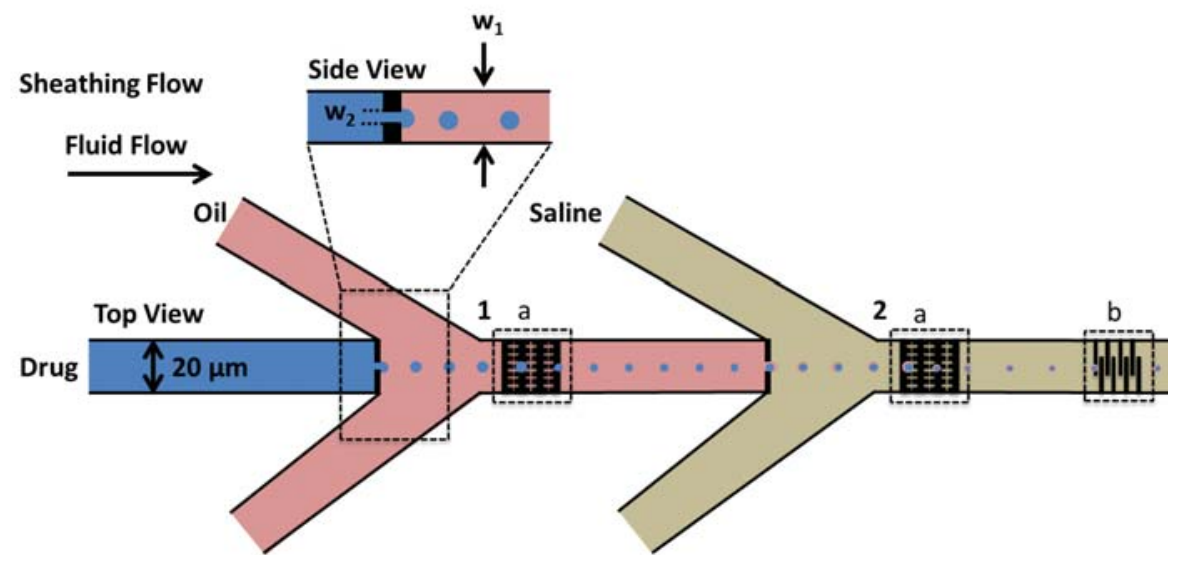

Figure A.3 Microchannel design for the chemotherapy drug emulsion formation with flow focusing and sheathing flow; appropriate scale not reflected. Dashed boxes (a) and (b) correspond to the placement of the electrodes for electrodispersion and dielectrophoretic micropumping, respectively. $\mathrm{w}_{2}$ is the orifice depth $4 \mu \mathrm{m}$, and $\mathrm{w}_{1}$ is the width of the junction $20 \mu \mathrm{m}$. The aqueous drug solution flows through the microchannel until it is dispersed into the continuous oil phase at junction 1 . At the junction 1 orifice, drug-in-oil droplets are formed and flow until they are then dispersed into the saline phase at junction 2 to achieve an oil sheathing of the drug droplet in the continuous saline phase. Double emulsion chemotherapy droplet is produced via the second orifice. See Figure A.4 for the COMSOL simulation of this fluid flow.

The droplet formation behavior is mainly determined by capillary number (Ca), which represents the balance between the viscous forces and interfacial tension at the surface of two fluids as defined in Equation 1. In Equation 1, $\mu$ is the viscosity of the continuous phase, $\mathrm{V}$ is the characteristic velocity, and $\mathrm{Y}$ is the surface tension. 
Capillary number is the most appropriate dimensionless number to describe droplet formation behavior because Reynolds number and Weber number are less significant in FF (W. Lee et al., 2009). In addition to Ca, the flow rate ratio ( $\varphi$ ), the viscosity ratio ( $\lambda$ ), and the expansion ratio (A) in Equations 2-4 (W. Lee et al., 2009) are also parameters to govern droplet formation because they balance viscous stresses and exerted shear stresses which result in droplet thread trajectory and velocity profile. In Equation 2, $\mathrm{Q}_{\mathrm{d}}$ and $\mathrm{Q}_{\mathrm{c}}$ are flow rate of dispersed phase and continuous phase, respectively. In Equation 3, $\mu_{\mathrm{d}}$ and $\mu_{\mathrm{c}}$ are viscosity of dispersed phase and continuous phase and $\mathrm{w}_{1}$ and $\mathrm{w}_{2}$ are depth of outside and inside orifice.

$$
\begin{gathered}
C a=\frac{\mu V}{\gamma} \\
\varphi=\frac{Q_{d}}{Q_{c}} \\
\lambda=\frac{\mu_{d}}{\mu_{c}} \\
\mathrm{~A}=\frac{w_{1}}{w_{2}}
\end{gathered}
$$

Accordingly, the size of droplets changes by capillary number due to viscous force and the interfacial tension. While flow rate ratio affects the droplet formation behavior significantly, the viscosity ratio has a relatively weak impact on the droplet diameter compared to capillary number $(\mathrm{Ca})$ since the flow rate is associated with characteristic velocity of $\mathrm{Ca}$. In addition, as the expansion ratio between the orifice and the junction depth decreases, a longer thread of smaller droplets is achieved, which is desired in the drug delivery microdevice. Much remains to be learned in regards to 
the underlying physics in the system as well as the stability of the droplet threads over long operation times. Therefore, a few assumptions were made in the FF designs of the microdevice. These assumptions include the combination of the FF droplet formation with the electrodispersion design to decrease droplet size, increases velocity of droplets, as well as with regards to the continuous robustness of producing droplets over long operation times (days) of the device.

Research has also been conducted in the related field of electrically manipulated emulsifications (H. Kim et al., 2007; Zagnoni et al., 2009; Zagnoni et al., 2010). Electric fields with a resonance frequency between $10 \mathrm{~Hz}$ and $10 \mathrm{MHz}$ have been used as a separation method to remove water dispersed in oil for applications in the petroleum industry. This data is applicable to droplets in the drug delivery microdevice since the frequency-modulated electric field utilized can be used as a deformation tool for emulsion droplets (Zagnoni et al., 2009; Zagnoni et al., 2010). Electric fields have also been used to focus and space nano and micro emulsions/particles in microchannels of $50 \mu \mathrm{m}$ (width)* $50 \mu \mathrm{m}$ (length)* $61 \mu \mathrm{m}$ (height) orifice dimensions in DC fields (H. Kim et al., 2007). H. Kim et al. studied electrospray emulsification and produced emulsion $<1 \mu \mathrm{m}$ in diameter with $\sim 2 \%$ of size distribution at a field of $1.4 * 10^{2}-5.5^{*} 10^{3} \mathrm{~V} / \mathrm{m}$, and also Arya et al. successfully synthesized of hundreds of $\mathrm{nm}$ chitosan micro/Nano spheres for drug delivery application in $2.3 * 10^{5}-4.7 * 10^{5} \mathrm{~V} / \mathrm{m}$. Furthermore, Mejia et al. formed wax emulsions with high uniformity for water-proof painting, cosmetics, and adhesives that supports the idea of utilizing electric field (2.6-2.9 $\mathrm{kV}$ in $500 \mathrm{ml}$ wax mixture) in the production 
of fine emulsions. Compared to these literature values of the applied electric fields, the device operating condition of $5^{*} 10^{6}-10^{7} \mathrm{~V} / \mathrm{m}$ is several orders are higher. The electric energy acting on the particles cause the water-oil interface to charge such that it behaves as a capacitor, which leads to a tip of Taylor cone which enables tiny droplets and narrow size distribution $2 \%$ (H. Kim et al., 2007).

Castellation configuration electrodes (Zagnoni et al., 2009; Zagnoni et al., 2010) were designed for electrodispersion because this design resulted in the highest localized electric field in the z-dimension, which has the potential to most efficiently manipulate the droplets and minimize deformation in the $\mathrm{x}$ - and $\mathrm{y}$ - directions. These are placed $200 \mu \mathrm{m}$ downstream from both junction 1 and junction 2 as shown in Figure A.3(a). This electric field energy does two things: a) it breaks apart the droplets from hundreds of nanometers in diameter into the more effectively adsorbed size of $<100$ $\mathrm{nm}$ and b) it spatially disperses the droplets in the continuous phase in order to minimize coalescence as droplets travel forward in microchannel.

In order to simulate behaviors in the drug delivery microdevice, COMSOL 4.2 was used to simulate the $3 \mathrm{D}$ electric field gradient of the electrodispersion design in Figure A.4, which is castellated gold electrodes of gap width $1 \mu \mathrm{m}$ (x-direction), width $2 \mu \mathrm{m}$ (y-direction), and thickness $20 \mathrm{~nm}$ (z-direction). The electrostatics module with the electrostatic potential (Equation 5), a relationship between electric displacement and the electric field (Equation 6), and Gauss's law (Equation 7) were used to simulate the electric field gradient in a fluid medium of saline.

$$
\vec{E}=-\vec{\nabla} V
$$




$$
\begin{aligned}
& \vec{D}=\varepsilon_{0} \vec{E}+\vec{P} \\
& \vec{\nabla} \cdot \vec{E}=\frac{\rho_{V}}{\varepsilon_{0}}
\end{aligned}
$$

In Equations $5-7, \vec{E}$ is the electric field, $\mathrm{V}$ is the electric potential, $\vec{D}$ is the electric displacement, $\varepsilon_{0}$ is the permittivity of a vacuum, $\vec{P}$ is the electric polarization and was assumed to be zero in the fluid medium, and $\rho_{\mathrm{V}}$ is the space charge density. Combining Equations 5-7 with zero electric polarization gives

$$
\vec{\nabla} \cdot\left(\varepsilon_{0} \varepsilon_{r} \vec{E}\right)=\rho_{V}
$$

Equation 8 is the governing equation used in to simulate the electric field gradient of the electrodispersion electrode design (Figure A.3). Equation 8 is modified slightly and employed in COMSOL as

$$
\nabla E=\sqrt{\frac{\partial^{2} E}{\partial x^{2}}+\frac{\partial^{2} E}{\partial y^{2}}+\frac{\partial^{2} E}{\partial z^{2}}}
$$

In COMSOL, the physical properties for water were altered slightly to simulate the saline such as $8.9 \times 10^{-4} \mathrm{~Pa} \cdot \mathrm{s}$ dynamic viscosity and 80 relative permittivity. The physical properties used for poppy seed oil were dynamic viscosity $5.58 \times 10^{-2} \mathrm{~Pa} \cdot \mathrm{s}$ and relative permittivity of 4 . Poppy seed oil is used to decrease the immediate toxicity of the chemotherapy drug (Pai et al., 2003) as it enters the tissue, and saline is used to carry the sheathed droplets and match tissue isotonicity during injection. Gold was the material used for the electrodes, and PDMS was the material used to form the orifices at the microchannel junctions (see Figure A.2 for orifice design) as reported in the literature (W. Lee et al., 2009). The initial flow rates in the microchannels were chosen 
to be $0.01 \mu \mathrm{L} / \mathrm{min}$ for the drug, $0.1 \mu \mathrm{L} / \mathrm{min}$ for the poppy seed oil, and $1 \mu \mathrm{L} / \mathrm{min}$ for the saline. These initial flow rate values were chosen based on Zagnoni et al. 2009 and 2010.

The fluid flow velocity in the FF microchannel was modeled with the laminar flow module employing the Navier Stokes equation and the continuity equation, which were simplified by assuming a steady-state system and an incompressible fluid as follows:

$$
\begin{gathered}
\rho(v \cdot \nabla) v=-\nabla p+\nabla\left(\mu\left(\nabla v+\nabla^{T} v\right)\right)+F_{S} \\
\nabla \cdot v=0
\end{gathered}
$$

In Equations 10 and $11, \rho$ is the fluid density, $v$ is the fluid velocity, $t$ is time, $p$ is the pressure, $\mu$ is the viscosity, and $F_{S}$ is the volumetric force on the fluid resulting from surface tension.

Two types of fluidic conditions of water (to represent drug and saline) and oil were employed, and the velocity of the formed emulsion droplets were calculated from the summation of pressure driven flow velocity and electro-osmotic velocity. The electro-osmotic flow velocity was calculated from Smoluchowski slip velocity equation via a wall boundary condition on the microchannel and added to simulate the velocity of the chemotherapy droplets in the field created by the electrodispersion, Figure A.3. The zeta potential for PDMS was assumed at $-0.1 \mathrm{~V}$ (Kirby and Hasselbrink Jr., 2004). The boundary condition for electrical potential was an applied DC field of $10 \mathrm{~V}$ and ground across each pair of electrodes. The velocities without electrodes were calculated from pressure driven flow in the $\mathrm{x}-, \mathrm{y}-$, and $\mathrm{z}$-direction and 
expressed with $u_{i}$. The governing equations for electro-osmotic flow used for this simulation are Equation 12-16 below,

$$
\begin{aligned}
& \mu_{E}=\frac{\varepsilon_{r} \varepsilon_{0} \zeta}{\eta} \\
& v_{E O F, i}=\mu_{E} \cdot \vec{E}_{i} \quad \text { where } \mathrm{i}=\mathrm{x}, \mathrm{y}, \mathrm{z}
\end{aligned}
$$

In Equations 12 and 13, $\mu_{\mathrm{E}}$ is electrophoretic force, $\varepsilon_{\mathrm{r}}$ is the relative permittivity of the fluid, $\varepsilon_{0}$ is the permittivity of a vacuum, $\zeta$ is the zeta potential, $\eta$ is the dynamic viscosity, VEOF, is the velocity due to electro-osmotic flow (EOF), and $\vec{E}$ is the electric field. The normal EOF velocity and the total velocity of the droplets are given as

$$
\begin{aligned}
& v_{\text {norm }}=\sqrt{v_{E O F, x}^{2}+v_{E O F, y}^{2}+v_{E O F, z}^{2}} \\
& v_{\text {total }}=\sqrt{v_{x, \text { total }}^{2}+v_{y, \text { total }}^{2}+v_{z, \text { total }}^{2}}
\end{aligned}
$$

The EOF velocity is related to the total velocity by,

$$
v_{i, \text { total }}=u_{i}+v_{E O F, i}^{2} \text { where } \mathrm{i}=\mathrm{x}, \mathrm{y}, \mathrm{z}
$$

The total velocity is displayed in the simulation scale bar next to each COMSOL diagram in Figures A.4 and A.5.

Figure A.4 shows the electric field gradient with and without the droplets. The maximum electric field gradient without droplets is $1.1 \times 10^{7} \mathrm{~V} / \mathrm{m}$ and the maximum electric field gradient with droplets is $2.3 \times 10^{7} \mathrm{~V} / \mathrm{m}$. This difference is because when the droplets pass in between the two electrodes, the droplets have an induced field, which influences the applied field gradient by reducing the gap over which the potential acts. The electric field is tuned to the resonant frequency of the droplets in 
order to break apart the $\sim 500 \mathrm{~nm}$ droplets into $<100 \mathrm{~nm}$ droplets as well as distribute the droplets spatially within the continuous fluid phase.

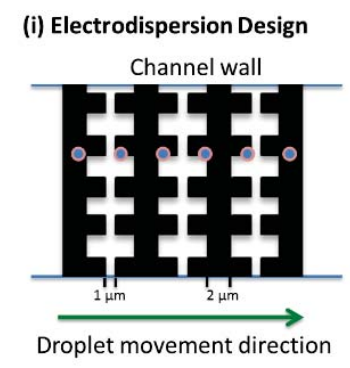

(iii) Velocity $(z=10 \mu \mathrm{m})$ with field off

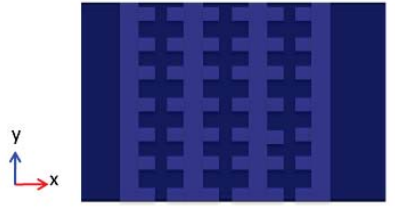

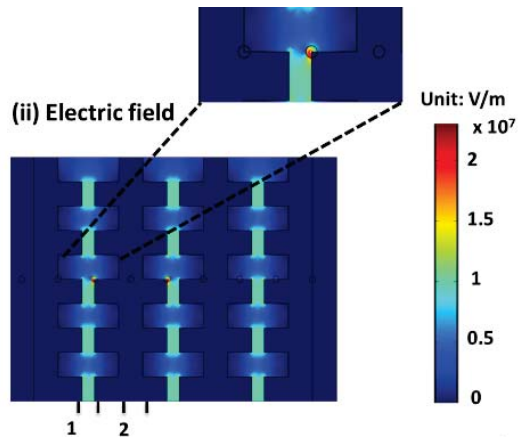

$\mu \mathrm{m} \mu \mathrm{m}$

Unit: $\mathrm{mm} / \mathrm{s}$

(iv) Velocity $(\mathrm{z}=10 \mu \mathrm{m})$ with field on

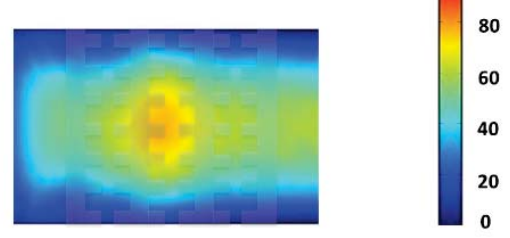

Figure A.4 COMSOL simulations of the electric field gradient as well as the velocity profiles (without and with the E field active) at the midpoint ( $\mathrm{x}, \mathrm{y}$ plane at $\mathrm{z}=10 \mu \mathrm{m}$ height) of the microchannel. (i) Channel level view of electrodes with cartooned drug droplets, (ii) electric field magnitude, (iii) fluid velocity above the inactivated electrodispersion electrodes at junction 1 (roughly $0.056 \mathrm{~mm} / \mathrm{s}$ ), and (iv) fluid velocity once the electric field is activated (roughly $73.0 \mathrm{~mm} / \mathrm{s}$ ). The maximum observed electric field strength during fluid flow is $2.3 \times 10^{7} \mathrm{~V} / \mathrm{m}$.

The drug delivery microdevice is designed so that it can be easily fabricated with standard UV-photolithographic methods in a Class 100 or greater cleanroom. The FF geometry integrated within the microchannel design is fabricated in poly (dimethylsiloxane) (PDMS) using standard printed masks, UV soft photolithography 
techniques, and multilayer alignment of the channels from one layer to the next. The electrodes can be fabricated on a silicon or glass support via photoresist masking followed by deposition of a $10 \mathrm{~nm}$ titanium layer then a $10 \mathrm{~nm}$ gold layer by electronbeam evaporation.

Each layer of the molded channels can be sealed via oxygen plasma bonding procedures. PDMS is hydrophobic so that oil wets the walls, but surface treatment defines the required hydrophobic and hydrophilic patterns depending on fluid phases used. The dispersed drug phases will be hydrophilic (viscosity, $\mu_{\mathrm{c}}=6 \mathrm{mPa} \cdot \mathrm{s}$ ) and a biocompatible surfactant will be mixed with either the continuous phase at a concentration approximately 2.5 times greater than the critical micelle concentration (CMC) which is a moderate surfactant concentration and is favorable to test a wide flow rate range (W. Lee et al., 2009). The presence of the surfactant does not substantially change the viscosity as evidenced by W. Lee et al.

A positive displacement chamber pump connected across a membrane to a pressurized canister will be used to drive the fluids from each inlet into the FF microchannels. Separate micropumps will connect to each solution reservoir such that flow in each channel can be separately controlled to achieve a feedback controlled fluidic system that can be worn on a wrist (Lima et al., 2004). Pressure drops for flows between 0.01 and $1 \mu \mathrm{L} / \mathrm{min}$ are not expected to cause deformation of microchannels due to either high-pressure injection or the PDMS elasticity used in our drug microdevice (Soller et al., 2011). If necessary, Thermoset Polyester (TPE) would be 
the best alternative material of PDMS due to its high rigidity and suitability with droplet microfluidics (Soller et al., 2011).

In order for a stable, efficient, and continuous small dosage of drug delivery, optimized selection of a surfactant, microfabrication condition, and pumping system are discussed. The unique combination of FF and electrodispersion to generate drug droplets protected by a sheathing layer of biocompatible poppy seed oil is described. Further, the electric field and fluid flow conditions were simulated and results used to optimize the design. The droplets exiting the flow focusing and electrodispersion region must then be accelerated in the drug delivery microdevice channel in order to generate a high enough pressure difference for the fluid to exit the microneedles into the dermis of the skin to achieve drug delivery.

\section{A.2.2 Dielectrophoretic Pumping}

Traveling wave DEP (twDEP) is incorporated into the drug delivery microdevice in order to accelerate the chemotherapy droplets as they travel to an array of microneedles for painless injection into the body. DEP is an efficient nondestructive way to manipulate bioparticles (Cheng et al., 2011), and twDEP is being investigated as a possible drug delivery technique (Bunthawin et al., 2010). The electrode configuration consists of an array of parallel rectangular electrodes configured in an intercalated pattern as shown in Figure A.1(ib). The intercalated configuration of the electrodes facilitates horizontal movement of particles when a non-uniform AC field is applied that is offset by $90^{\circ}$ with each successive electrode. This causes the field 
maxima to travel in waves down the array of electrodes thus driving the particle forward. Typically the spacing between the electrodes is fixed to the width of one single electrode with the optimal width of an electrode being close to the diameter of the target particle. The spacing between the electrodes is usually $10 \mu \mathrm{m}$ to $50 \mu \mathrm{m}$ and remains constant (Lin \& Yeow, 2007).

Parallel electrodes are used for collecting, transporting, and/or separating particles. For the drug delivery microdevice, we will be using the parallel electrodes for transporting the oil-sheathed chemotherapy droplets. To help facilitate transportation the frequency and conductivity of medium is chosen specifically to induce the largest positive DEP force on the dielectric particles. Positive DEP is the movement of particles up the electric field gradient. When the AC field is applied to the electrodes a dipole moment is induced in the particles and the time-average DEP force, $\left\langle F_{D E P}\right\rangle$, is given as (Pethig, 2010)

$$
\left\langle F_{D E P}\right\rangle=2 \pi \varepsilon_{m} R^{3}\left\{\operatorname{Re}\left[f_{c m}\right] \nabla E^{2}+\operatorname{Im}\left[f_{c m}\right] \sum E^{2} \nabla \phi\right\}
$$

In Equation $17 \varepsilon_{\mathrm{m}}$ is the absolute permittivity of the medium, $\mathrm{R}$ is the radius of the particle, $\mathrm{f}_{\mathrm{cm}}$ is the Clausius-Mossotti factor, $\mathrm{E}$ is the amplitude of the electric field, and $\phi$ is the phase. The Clausius-Mossotti factor is related to the polarizability of the particle and $\sum E^{2} \nabla \phi$, is the summation of the magnitude and phase of each field component. The Clausis-Mossotti factor, $f_{c m}$, for a spherical, homogeneous particle is given as (Pethig, 2010)

$$
f_{c m}=\frac{\underline{\varepsilon}_{p}-\underline{\varepsilon}_{m}}{\underline{\varepsilon}_{p}+2 \underline{\varepsilon}_{m}}
$$


In Equation $18 \underline{\varepsilon}_{p}$ is the complex permittivity of the particle and $\underline{\varepsilon}_{\mathrm{m}}$ is the complex permittivity of the medium defined as (Pethig, 2010)

$$
\begin{aligned}
& \underline{\varepsilon}_{p}=\varepsilon_{p}+\frac{\sigma_{p}}{j \omega} \\
& \underline{\varepsilon}_{m}=\varepsilon_{m}+\frac{\sigma_{m}}{j \omega}
\end{aligned}
$$

In Equation 19 and $20 \varepsilon_{\mathrm{p}}$ is the absolute permittivity of the particle, $\sigma_{\mathrm{p}}$ is the conductivity of the particle, $\sigma_{\mathrm{m}}$ is the conductivity of the medium, $\omega$ is the angular frequency and $\mathrm{j}$ is the imaginary number. It is important to note that the DEP force for twDEP is dependent on both the real and imaginary part of the Clausius-Mossotti factor which causes particles to experience both an in-phase force (real part) and the out of phase force (imaginary part). Classical DEP force is only dependent on the real portion of the Clausius-Mossotti factor, $\left\langle F_{D E P}\right\rangle=2 \pi \varepsilon_{m} R^{3} \operatorname{Re}\left[f_{c m}\right] \nabla|E|^{2}$.

Further, the assumption that the oil-sheathed droplets of aqueous drug can be represented as a first approximation homogeneous particle is acceptable because the conductivity of the oil layer is substantially different $(\approx 1 \mathrm{nS} / \mathrm{m})$ from the supporting saline medium that the Clausius-Mossotti factor is nearly $0.50-3.36^{*} 10^{-8} \mathrm{i}$ and varies very little $\left(4.8 \times 10^{-8} \%\right)$ over the anticipated frequency range of interest from $1 \mathrm{~Hz}$ to $10 \mathrm{MHz}$ (Durr et al., 2003; Felten et al., 2008). Since twDEP is being used to transport chemotherapy droplets, it's important to have an expression that relates the particle electrophoretic mobility with twDEP force. The DEP force can also be written utilizing the zeta potential $\zeta_{\mathrm{p}}(\mathrm{Kang} \& \mathrm{Li}, 2009)$, 


$$
\vec{F}_{D E P}=6 \pi \zeta_{p} \varepsilon_{r} R E
$$

The DEP force written in this form allows it to be related to the electrophoretic mobility of the particle given as (Kang \& Li, 2009)

$$
\begin{gathered}
\mu_{E}=\frac{\varepsilon_{r} \varepsilon_{0} \zeta_{p}}{\eta} \\
v_{D E P}=\frac{R^{2} \varepsilon_{r} \varepsilon_{0} \operatorname{Re}\left[f_{c m}\right]}{3 \eta} \nabla E
\end{gathered}
$$

In equations $21-23 \zeta_{\mathrm{p}}$ is the zeta potential, $\varepsilon_{\mathrm{r}}$ is the relative permittivity of the medium, $\mathrm{R}$ is the radius of the particle, $\mathrm{E}$ is the magnitude of the electric potential, $\varepsilon_{0}$ is the permittivity of free space, $\operatorname{Re}\left[\mathrm{f}_{\mathrm{cm}}\right]$ is the real-part of the Clausis-Mossotti factor, and $\eta$ is the dynamic viscosity of the medium.

Parallel electrodes were added to the end of the microchannels, Figure A.2(ib), to increase the velocity of the chemotherapy droplets to ensure the droplets reach the microneedles at the end of the device with sufficient velocity to penetrate the dermis. The desired velocity at the microneedle tips was $10 \mu \mathrm{m} / \mathrm{s}$ based on modeling of transdermal delivery (Lv et al., 2006). COMSOL was used to simulate the electric field gradient via the conservation of electrical potential between the insulating channel walls and the velocity of the droplets via the Navier-Stokes (Equation 12) and conservation relationships (Equation 13). Equations 5-9 developed in section 2.1 were used to obtain the electric field gradient with and without droplets in the channel as shown in Figure A.5. Droplets were defined as separate spherical 


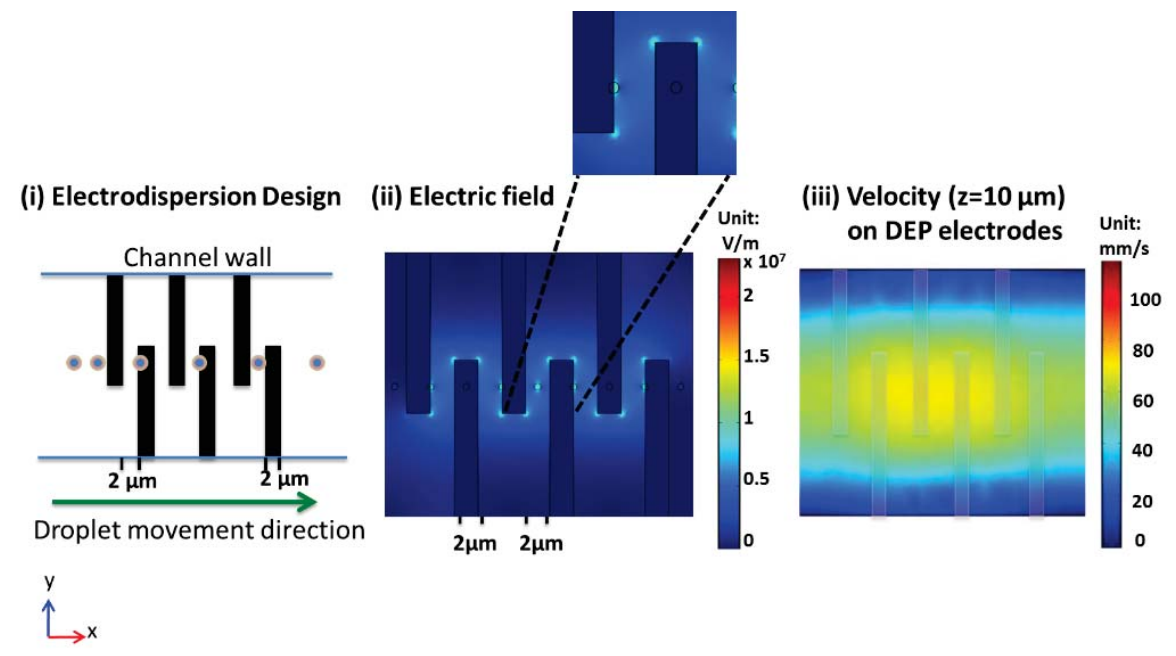

Figure A.5 COMSOL simulation of traveling wave dielectrophoretic pumping electrodes before and during fluid flow in the microchannel. The combined total length of the electrodes including gaps is $22 \mu \mathrm{m}$ (i) channel level view of electrodes, (ii) normalized electric field strength from twDEP electrodes, (iii) velocity distribution in the channel view. In (i) when the twDEP electrodes are energized at an instantaneous half cycle with max potential of $10 \mathrm{Vpp}$ to ground at subsequent electrodes. The maximum observed electric field strength before fluid flow is $7.2 \times 10^{6} \mathrm{~V} / \mathrm{m}$, and the maximum electric field strength during fluid flow is $1.1 \times 10^{7} \mathrm{~V} / \mathrm{m}$. The droplet fluid velocity was $70 \mathrm{~mm} / \mathrm{s}$ in this DEP pumping region at $\mathrm{z}=10 \mu \mathrm{m}$.

fluids with the laminar flow module in COMSOL. The material selected in COMSOL for the particles was oil as previously described in section 2.1 and modified water to represent saline. It can be seen in Figure A.5 that the electric field gradient decreases by $65 \%$ when the droplets are present because of the change in the electric charge of the dielectric oil layer. Further, the droplets align along the centerline of the electrode 
configuration because the DEP force is directed toward the symmetric centerline. This phenomenon is observed because electric field interact with droplets and induce force due to the dielectric property difference between immiscible phases (water and oil) since free charges can accumulate on the interface between inner and outer fluid. The droplet equilibrium height is 10 microns above the bottom of the channel. DEP forces are predicted to be on the order of $152 \mathrm{pN}$ for the $10 \mathrm{Vpp}$ field over the $22 \mu \mathrm{m}$ electrodes.

The electrophoretic mobility was used to simulate the velocity of the chemotherapy droplets in the field created by the dielectrophoretic pumping electrodes. In this drug delivery microdevice system, the droplet is a chemotherapy drug coated with poppy seed oil in a continuous phase of saline. In order to look at electric field effect, electrophoretic mobility was an added term.

In Figure A.5, the fluid velocities increased by over 5000 times from $0.021 \mathrm{~mm} / \mathrm{s}$ to $109 \mathrm{~mm} / \mathrm{s}$ by implementing dielectrophoretic pumping at $10 \mathrm{Vpp}$ via the electrode designs in the COMSOL simulation. Comparing without and with dielectrophoretic pumping (Figure A.4(iv) and Figure A.5(iii), respectively), the velocities with electrodispersion and DEP pumping electrodes remarkably changed as demonstrated in Figure A.6. These increases in velocity are necessary to move the drug droplets forward to the microneedles for injection. 


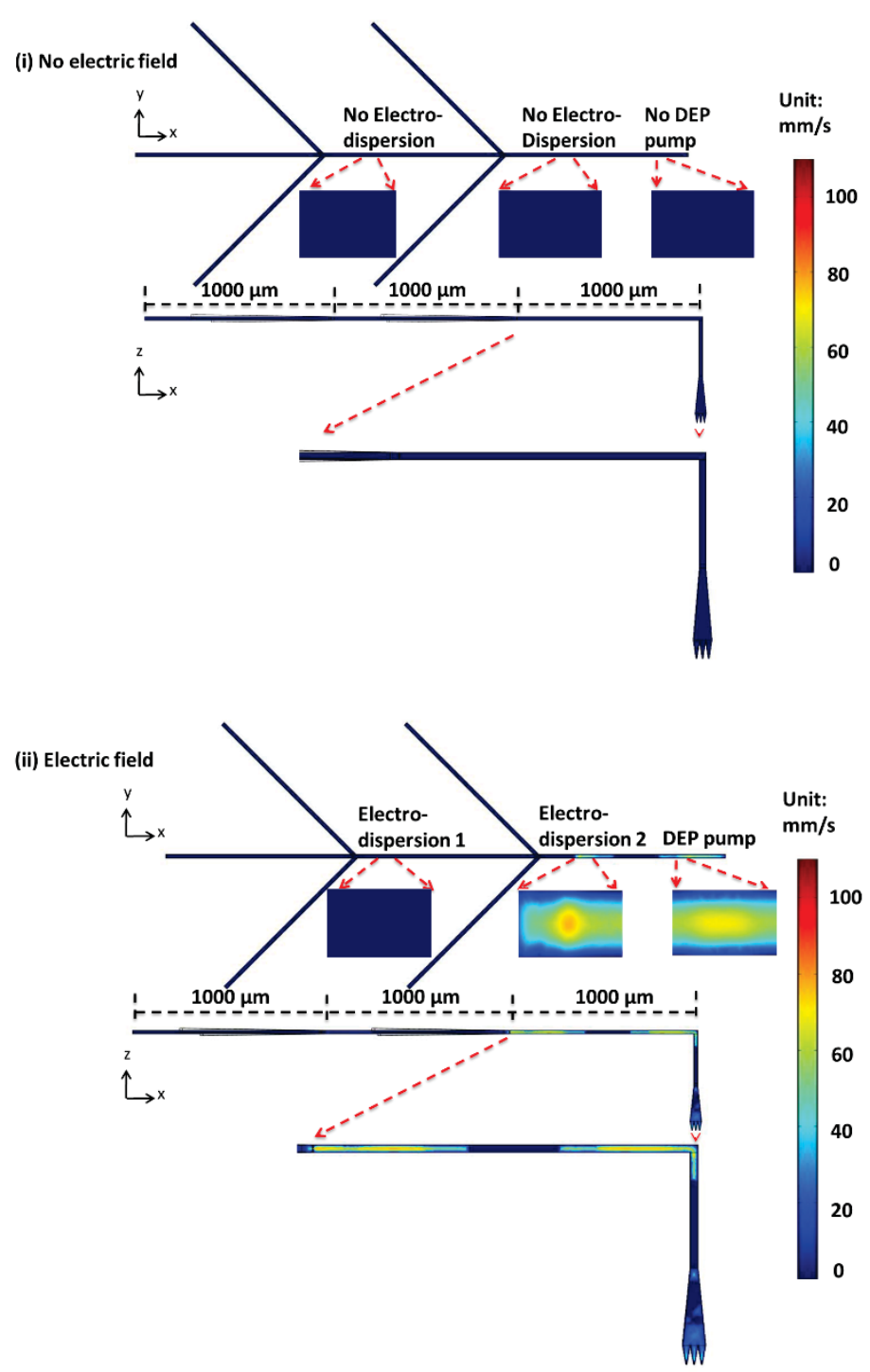

Figure A.6 The top of each simulation shows $\mathrm{x}-\mathrm{y}$ plane, top view, and bottom of each simulations shows z-x plane to display overall velocity profile. COMSOL simulation of fluid velocity without (i) and with (ii) dielectrophoretic pumping electrodes. Interdigitated dielectrophoretic pumping electrodes were added to the end of the microchannel to increase the velocity of the chemotherapy drug droplets to ensure sufficient velocity for microneedle injection. (i) The maximum fluid velocity inside the microchannel without electrodes was $0.025 \mathrm{~mm} / \mathrm{s}$ reached at junction 2 , (ii) the 
maximum fluid velocity with electrodes was $109 \mathrm{~mm} / \mathrm{s}$ also at junction 2 . The magnified sections for (i) and (ii) show that the fluid velocity substantially increases from $0.0056 \mathrm{~mm} / \mathrm{s}$ to $35 \mathrm{~mm} / \mathrm{s}$ at the end of the microchannel once the pumping electrodes are added.

\section{A.2.3 Microneedles}

The skin is a common area for drug delivery, and offers advantages over other non-invasive drug delivery techniques. Drug delivery through the skin avoids drug metabolism by enzymatic reactions and the gastro-intestinal system, has the potential for continuous drug delivery (Migalska et al., 2011), and facilitates reaching circulating tumor cells (CTCs) that metastacized from their origin. Hypodermic needles are commonly used as the dominant method for transdermal drug delivery for gastric cancer treatment. Needles are painful, inconvenient and require professional administration for each dose (P.M. Wang et al., 2006). Another problem is that drugs are delivered all at once which causes an immediate spike in drug concentration profiles, which then rapidly diminishes. This can cause physiological instabilities, other dosage-related side-effects and can potentially fail to completely eradicate the tumor and/or CTCs (Zahn et al., 2004). To overcome these drawbacks, this work proposes a novel drug delivery microdevice amenable to feedback control; the device integrates microchannel hydrodynamic flow focusing, electrodispersion to decrease drug droplet size, dielectrophoretic pumping, and microneedles together in one 
microdevice to deliver chemotherapy drugs in the form of droplets to a gastric cancer patient.

The skin has four layers: (1) the stratum corneum, principal barrier composed of corneocytes, (2) epidermis, (3) dermis, and (4) subcutaneous tissue (EscobarChavez et al., 2011). The four layers of the skin are barriers to transdermal drug delivery and microneedles are used as physical enhancers for transdermal drug delivery. They are designed to increase the permeability of the skin up to four orders of magnitude, so that drug passage through the stratum corneum and outer most layer of the epidermis becomes simple (Escobar-Chavez et al., 2011; K. Lee et al., 2011). Microneedles allow for drugs to be delivered across the skin in four ways: (1) "pokewith-patch", this method uses a solid microneedle array to penetrate the skin creating micropores, the microneedle array is removed and drugs are delivered through the micropores via a transdermal patch, gel or solution; (2) "coat-and-poke", this method coats an array of microneedles with a drug and inserts the coated microneedles into the skin; and (3) "poke-and-release" embeds the drug molecules into the structure of polymer, biodegradable microneedles and inserts them into the skin; and (4) "pokeand-flow", uses hollow microneedles to insert liquid drugs into the skin (Migalska et al., 2011). There are advantages and disadvantages to each approach and these vary with the application. Micropores created by microneedle penetration last for more than a day when left covered and they last for less than 2 hours when left uncovered. Microneedles that dissolve under the skin are perceived as the safest with the least chance of prolonged irritation (K. Lee et al., 2011). Methods 1, 2, and 3 are best suited 
for applications where a one-time dose or daily dose of drug is desired. However, for the chemotherapy drug delivery microdevice, method 4 is optimal. The microneedles would remain inserted in the skin and held in place via a wristband as shown in Figure A.1.

Microneedles can be inserted into the skin easier than hypodermic needles because stress on the skin is inversely proportional to the area of the top (Zahn et al., 2004). Microneedles require sharpness to overcome stress forces on the skin surface as well as strength against fracturing, bending, and buckling, sufficient flow rate, and biocompatibility of the needle material (Nguyen \& Wereley, 2006). The insertion force can be lowered by utilizing kinetic energy such as vibration which can reduce the force by as much as 30\% (Nguyen \& Wereley, 2006), and provides an increase in infusion flow rate (P.M. Wang et al., 2006). Retracting the microneedles after insertion by approximately 100-300 $\mu \mathrm{m}$ also achieves a much greater flow rate (P.M. Wang et al., 2006).

Microneedles have been shown to increase the transdermal delivery of many molecules including aminovulinic acid, anthrax, bovine serum albumin, desmospressin, erythropoirtin, meso-tera(N-methyl-4-pyridyl)porphine tetra tosylate, ovalbumin, plasmid DNA, low molecular weight tracers to proteins, and nanoparticles. Insulin is the most widely studied drug with microneedles and enhanced skin permeability has been reported in vivo and in vitro (Donnelly et al., 2011; EscobarChavez et al., 2011). Recently researchers have looked into using microneedles fabricated from maltose to deliver methotrexate to rats via iontophoresis to treat 
cancer. The result of this study was a synergistic 25 -fold increase of drug delivery (Escobar-Chavez et al., 2011).

Microneedles are a painless drug delivery method and create larger transport pathways for larger molecules; our device extends this to nanometer droplets. The painless characteristics of microneedles allow them to overcome the limitation of hypodermic needles. Advantages of microneedle technology are that the transport mechanisms are not dependent on the diffusion into the tissue, placement in the epidermis allows for drugs to reach target areas more readily, while only penetrating the stratum corneum without piercing nerve endings thus reducing pain, infection, or other injury. The microneedles are nontoxic, minimally invasive, can be massproduced for a range of materials such as silicon dioxide and polymers, are easily disposable/interchangeable, and can be made from biodegradable materials. Some disadvantages to using microneedles are local inflammation and skin irritation. Another disadvantage is that the microneedles may break and be left under the skin; to avoid this, the diameter of the microneedle should be smaller than the diameter of a hair, $\leq 50 \mu \mathrm{m}$ (Escobar-Chavez et al., 2011). In comparison hypodermic needles are inconvenient, not easily self-administered, and have poor targeted delivery because they have to be manually injected (P.M. Wang et al., 2006).

Our microdevice uses an array of microneedles, Figure A.7, to deliver drug-inoil microdroplets, applying the "poke-and-flow" method. Figure A.7(i) shows the design of a single microneedle wherein the diameter of hole in the microneedles is approximately $40 \mu \mathrm{m}$ which is sufficiently large to allow delivery of the oil sheathed 
drug droplets without disruptive shearing effects while simultaneously being large enough to avoid breakage in the skin. Figure A.7(ii) is color coded to show the array of microneedles for each of the chemotherapy drugs with blue representing epirubicin, red representing cisplatin, and green representing fluorouracil. Each microneedle is connected to its own flow-focusing channel as shown in Figure A.2. Figure A.7(iii) shows a photograph of an array of microneedles (Baek et al., 2011) fabricated from polylactic acid. The fluid emulsion velocities are approximately $35 \mathrm{~mm} / \mathrm{s}$ as they leave the DEP pumping electrode region. Once the emulsions enter their final descent, to the microneedles the midchannel linear velocity decreases to approximately 30 to $40 \mathrm{~mm} / \mathrm{s}$ as the channel expands followed by velocity increases within the microneedle tip, as constrained by the continuity equation.

Several methods are used to fabricate hollow microneedles and most are made from silicon or silicon-based materials. This drug delivery microdevice requires a relatively straight channel with minimal bends so that the microdroplets are not sheared and are delivered to the dermis intact. The pyramid-shaped microneedles were chosen because fabrication is simple and thus it has the best shape to achieve tip sharpness and strength (Moon \& Lee, 2003). The pyramid microneedles can be fabricated by an inclined LIGA process combining lithography, electroplating, and molding techniques. This process utilizes X-ray's directed towards a protective electroplated gold mask over a poly(methyl methacrylate) (PMMA) substrate on a silicon wafer (Moon \& Lee, 2003). 
As discussed in this section, optimal material, design, and operating conditions were gleaned from the literature. This information was combined to simulate electric field and hydrodynamic flow behaviors in each section of the microdevice. The design was iteratively optimized based on these simulation results and then integrated together into the drug delivery microdevice.

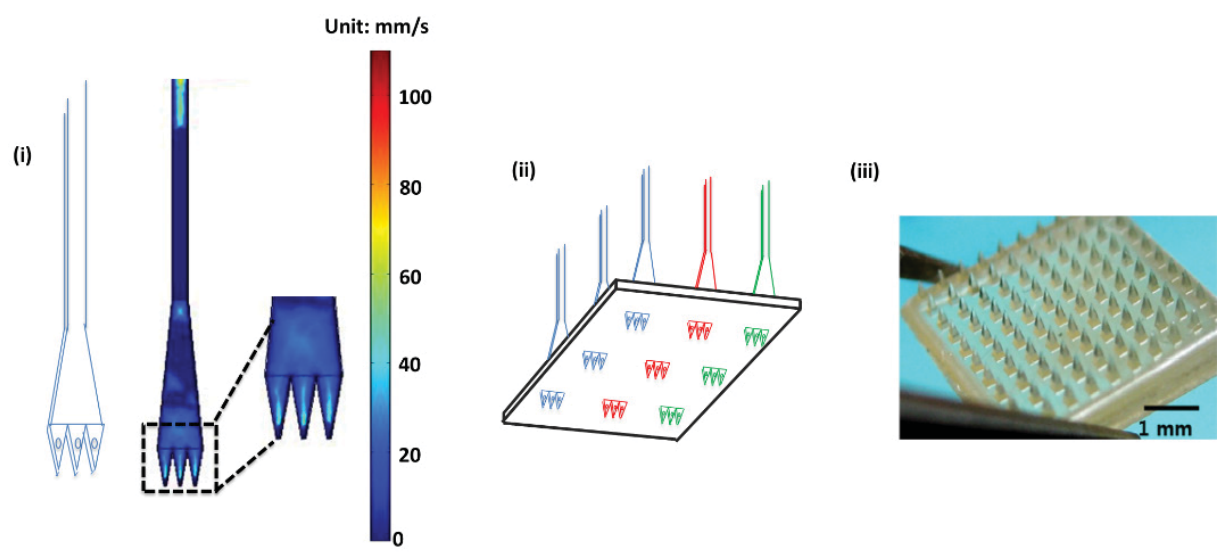

Figure A.7 Microneedle design to deliver chemotherapy emulsion to cancer patient, (i) pyramid-shaped hollow microneedle for delivery of 3 chemotherapy droplets (epirubicin, cisplatin, and fluorouracil) and the COMSOL simulation of the fluid velocity in the microneedle, (ii) microneedle array, and (iii) real image of a microneedle array. The initial velocities of the fluids in the microchannel were $4.2^{*} 10^{-}$ ${ }^{4} \mathrm{~m} / \mathrm{s}$ drug, $4.2 * 10^{-3} \mathrm{~m} / \mathrm{s}$ poppy seed oil, and $4.2^{*} 10^{-2} \mathrm{~m} / \mathrm{s}$ saline, and the exiting velocities from the microneedles is approximately $40 \mathrm{~mm} / \mathrm{s}$. In (iii) microneedles are made out of poly-lactic acid (Baek et al., 2011). 


\section{A.3 Integration of Technology into Fully Conceived Device}

This microfluidic drug delivery device will operate in sequence as described in sections 2.1 through 2.3. First, three different chemotherapy drugs, Epirubicin, Cisplatin, and Fluorouracil, will be separately dispersed into oil and then subsequently into saline by proven flow-focusing microchannel technology. Current state of the art results in this field suggest droplets formed will be approximately $500 \mathrm{~nm}$ in diameter. This design utilizes two stages of interdigitated electrodes on the bottom surface of the microchannel leaving the flow focusing junction in order to electrodisperse the droplets into $<100 \mathrm{~nm}$ droplets and to evenly disperse them spatially as they flow downstream to reduce coalescence. The oil-sheathed drug droplets are then pumped down the microchannel using traveling wave DEP technologies before flowing into an array of microneedles inserted into the dermis layer of the skin. This entire system is packaged inside of a small unit that can be worn on the wrist. The reservoirs for each

drug, poppy seed oil, saline, and pressurized air to pump from the reservoirs can be individually replaced based on usage. Further, integrated electronic feedback control and monitoring (not described here) can be utilized to monitor chemotherapy drug delivery into the dermis and subsequently the blood stream of a cancer patient. The main components of this microfluidic device were optimized from literature data and COMSOL simulations. 


\section{A.4 Conclusions and Perspectives for Future Integrated Microdevice}

\section{Technologies}

There is a great need for new technologies to effectively treat all forms of metastasized cancer. Gastric cancer provides a poignant example because patient symptoms typically do not arise until the cancer has progressed to stage IV. Any new technologies developed should increase the comfort level of patients as well as concurrently improve treatment efficacy or even eradicate the disease. The goal of this work was to develop a novel drug delivery system to effectively treat gastric cancer patients with minimal pain or lifestyle interruptions while undergoing treatment.

In addition, this work links together technologies that have been progressing in isolation from each other. For example, electrodispersion integrated with flow focusing and surfactant stabilization is a novel technique with the potential to produce droplets less than $100 \mathrm{~nm}$ in diameter. Smaller droplets are desired in diverse applications such as nanoparticle synthesis or pharmaceutical packaging. One key advantage of this combined technique is that it can be integrated into lab-on-a-chip devices provided the capillary number and volumetric flow rate ratio are optimized and the surfactant required for optimizing interfacial tension increases the long-term stability of droplets.

Traveling wave DEP is an advantageous technique for transport of droplets in microchannels, which has minimal power requirements and thus is ideal for portable microdevices operating on batteries. Incorporating traveling wave DEP electrodes into the drug delivery microdevice described increased the velocity of the droplets for 
optimal microneedle injection rates. Further, this technique has proven to be minimally disruptive to a particle, which is a key advantage with this adaption of the technology in the drug delivery microdevice.

Microneedles have been explored in many forms as physical enhancers for drug delivery. Within the drug delivery microdevice, the microneedle array was adopted to reduce pain and facilitate continuous delivery of the Epirubicin, Cisplatin, and Fluorouracil chemotherapy drug cocktail into the dermis. Based on evidence from previous studies, the pain level can be greatly reduced and the chemotherapy droplets reach their target areas in a more efficient manner thus reducing side effects.

For future work this drug delivery microdevice wrist system could be improved by incorporating a biosensor, in-line feedback control, and wireless reporting to measure the concentration and metabolites of the chemotherapy droplets in the blood stream, dynamically adjust dosage, and keep the primary care physician informed of progress. This will allow for real-time drug and treatment monitoring. The advantages of adding in this technology would be decreases in patient drug side effects, uniform maintenance of the critical drug concentration delivered to the gastric tumor and CTCs, increased treatment effectiveness, and increased patient comfort. 


\section{A.5 References}

1. Abismaïl, B., Canselier, J.P., Wilheml, A.M., Delmas, H., \& Gourdon, C. (1999). Emulsification by ultrasound: drop size distribution and stability. Ultrasonics Sonochemistry, 6, 1-2, (March 1999), pp.75-83, ISSN 1350-4177.

2. Aboud, M.J., Gassmann, M., \& McCord, B.R. (2010). The development of mini pentameric STR loci for rapid analysis of forensic DNA samples on microfluidic system. Electrophoresis, 31, 15, (August 2010), pp. 2672-2679, ISSN 0173-0835.

3. Alazzam, A., Stiharu, I., Bhat, R., \& Meguerditchian, A. (2011). Interdigitated comb-like electrodes for continuous separation of malignant cells from blood using dielectrophoresis. Electrophoresis, 32, 11, (June 2011), pp. 1327-1336, ISSN 0173-0835.

4. American Cancer Society. (n.d.). Cancer facts \& Figures 2012, In: Cancer.Org, August 2011, Available from: $<$ http://www.cancer.org/Research/CancerFactsFigures/CancerFactsFigures/ca cer-facts-figures-2011>.

5. Anna, S.L., Bontoux, N., \& Stone, H.A. (2003). Formation of dispersions using "flow focusing" in microchannels. Applied Physics Letters, 82, 3, (January 2003), pp. 364-366, ISSN 0003-6951.

6. Arya, N., Chakraborty, S., Dube, N., \& Katti, D.S. (2009). Electrospraying: A Facile Technique for Synthesis of Chitosan-Based Micro/Nanopsheres for 
Drug Delivery Applications. Journal of Biomedical Materials Research Part B: Applied Biomaterials, 88B, 1, (January 2009), pp. 17-31, ISSN: 1552-4973.

7. Baek, C., Han, M., Min, J., Prausnitz, M.R., Park, J.H., \& Park, J.H. (2011). Local transdermal delivery of phenylephrine to the anal sphincter muscle using microneedles. Journal of Controlled Release, 154, 2, (September 2011), pp. 138-147, ISSN 0168-3659.

8. Reprinted from Journal of Controlled Release, 154, Baek, C., Han, M., Min, J., Prausnitz, M.R., Park, J.H., \& Park, J.H., Local transdermal delivery of phenylephrine to the anal sphincter muscle using microneedles, pp. 138-147, Copyright (2011), with permission from Elsevier.

9. Balcer-Kubiczek, E.K., \& Garofalo, M.C. (2009). Molecular targets in gastric cancer and apoptosis, In: Apoptosis in Carcinogenesis and Chemotherapy, Chen, C.G. and Lai, P.B.S., pp. 157- 192, Springer Science + Business Media, Retrieved from $<$ http://www.crcnetbase.com/search/advanced $>$.

10. Bienvenue, J.M., Duncalf, N., Marchiarullo, D., Ferrance, J.P., \& Landers, J.P. (2006). Microchip-based cell lysis and DNA extraction from sperm cells for application to forensic analysis. Journal of Forensic Sciences, 51, 2, (March 2006), pp. 266-273, ISSN 0022-1198.

11. Bjerklie, D. \& Jaroff, L. (January 15, 2001). Beyond needles and pills, In: Time Magazine U.S., October 2011, Available from: < http://www.time.com/time/magazine/article/0,9171,998968,00.html>. 
12. Brammer, K.S., Choi, C., Oh, S., Cobb, C.J., Connelly, L.S., Loya, M., Kong, S.D., \& Jin, S. et al. 2009. Antibiofouling, sustained antibiotic release by $\mathrm{Si}$ nanowire templates. Nano Letters, 9, 10, (October2009), pp. 3570-3574, ISSN $1530-6984$.

13. Bunthawin, S., Wanichapichart, P., Tuantranont, A., \& Coster, H.G.L. (2010). Dielectrophoretic spectra of translational velocity and critical frequency for a spheroid in traveling electric field. Biomicrofluidics, 4, 1, (March 2010), ISSN 1932-1058.

14. Cheng, I.F., Chung, C.C., \& Chang, H.C. (2011). High-throughput electrokinetic bioparticle focusing based on a travelling-wave dielectrophoretic field. Microfluidics and Nanofluidics, 10, 3, (March 2011), pp. 649-660, ISSN 1613-4982.

15. Cleveland Clinic Foundation. (n.d.).What is Chemotherapy, In: Chemocare.com, September 2010, Available from: $<$ http://www.chemocare.com/whatis/how_do_the_doctors_decide_which.as $>$. 16. Donnelly, R.F., Majithiya, R., Singh, T.R.R., Morrow, D.I.J., Garlad, M.J., Demir, Y.K., Migalska, K., Ryan, E., Gillen, D., Scott, C.J., \& Woolfson, A.D. (2011). Design, optimization and characterisation of polymeric microneedle arrays prepared by a novel laser-based micromoulding technique. Pharmaceutical Research, 28, 1, (January 2011), pp. 41-57, ISSN 0724-8741. 17. Durr, M., Kentsch, J., Muller, T., Schnelle, T., \& Stelzle, M. (2003). Microdevices for manipulation and accumulation of micro- and nanoparticles 
by dielectrophoresis. Electrophoresis, 24, 4, (February 2003), pp. 722-731, ISSN 0173-0835.

18. Elman, N.M., Duc, H.L.H., \& Cima, M.J. (2009). An implantable MEMS drug delivery device for rapid delivery in ambulatory emergency care. Biomedical Microdevices, 11, 3, (June 2009), pp. 625-631, ISSN 1387-2176.

19. Escobar-Chavez, J.J., Bonilla-Martinez, D., Villegas-Gonzalez, M.A., MolinaTrinidad, E., Casas-Alancaster, N., \& Revilla-Vazquez, A.L. (2011). Microneedles: a valuable physical enhancer to increase transdermal drug delivery. Journal of Clinical Pharmacology, 51, 7, (July 2011), pp. 964-977, ISSN 0091-2700.

20. Farokhzad, O.C., Dimitrakov, J.D., Karp, J.M., Khademhosseini, A., Freeman, M.R., \& Langer, R. (2006). Drug delivery systems in urology - getting “smarter”. Urology, 68, 3, (September 2006), pp. 463-469,ISSN 0090-4295.

21. Felten, M., Staroske, W., Jaeger, M.S., Schwille, P., \& Duschl, C. (2008). Accumulation and filtering of nanoparticles in microchannels using electrohydrodynamically induced vertical flows. Electrophoresis, 29, 14, (July 2008), pp. 2987-2996, ISSN 0173-0835.

22. Ha, J.W., \& Yang, S.M. (1999). Breakup of a multiple emulsion drop in a uniform electric field. Journal of Colloid and Interface Science, 213, (May 1999), pp. 92-100, ISSN: 0021-9797.

23. Hamouda, T., Hayes, N.M., Cao, Z.Y., Tonda, R., Johnson, K., Wright, D.C., Brisker, J., \& Baker, J.R. (1999). A novel surfactant nanoemulsion with broad- 
spectrum sporicidal activity against bacillus species. Journal of Infectious Diseases, 180, 6, (December 1999), pp. 1939-1949, ISSN 0944-5013.

24. Hershock, D. (2006). Medical and Surgical Therapy for Gastric Cancer, In: Endoscopic Oncology, Faigel, D.O. and Kochman, M.L., pp. 173-181, $\begin{array}{llll}\text { Humana } & \text { Press, } & \text { Retrieved } & \text { from }\end{array}$ $<$ http://www.crcnetbase.com/search/advanced $>$.

25. Jensen, K. \& Lee, A. (2004). The science \& applications of droplets in microfluidic devices - Foreword. Lab On A Chip, 4, 4, (n.d.), pp. 31N-32N, ISSN 1473-0189.

26. Kang, Y. \& Li, D. (2009). Electrokinetic motion of particles and cells in microchannels. Microfluidics and Nanofluidics, 6, 4, (April 2009), pp. 431460, ISSN 1613-4982.

27. Kim, H., Luo, D.W., Link, D., Weitz, D.A., Marquez, M., \& Cheng, Z.D. (2007). Controlled production of emulsion drops using an electric field in a flow-focusing microfluidic device. Applied Physics Letters, 91, 13, (September, 2007), pp.133106, ISSN 0003-6951.

28. Kim, S.H., Kim, J.W., Cho, J.C., \& Weitz, D.A. (2011). Double-emulsion drops with ultra-thin shells for capsule templates. Lab Chip, 2011, 11, 18, (2011), pp. 3162-3166, ISSN 1473-0197.

29. Kiss, N., Brenn, G., Pucher, H., Wieser, J., Scheler, S., Jennewein, H., Suzzi, D., \& Khinast, J. (2011). Formation of O/W emulsions by static mixers for 
pharmaceutical applications. Chemical Engineering Science, 66, 21, (November 2011), pp. 5084-5094, ISSN: 0009-2509.

30. Kirby, B.J., \& Hasselbrink, E.F. (2004). Zeta potential of microfluidic substrates: 2. Data for polymers. Electrophoresis, 25, 2, (January 2004) pp. 203-213, ISSN: 0173-0835.

31. Lee, K., Lee, C.Y., \& Jung, H. (2011). Dissolving microneedles for transdermal drug administration prepared by stepwise controlled drawing of maltose. Biomaterials, 32, 11, (April 2011), pp. 3134-3140, ISSN 0142-9612.

32. Lee, W., Walker, L.M., \& Anna, S.L. (2009). Role of geometry and fluid properties in droplet and thread formation processes in planar flow focusing. Physics of Fluids, 21, 3, (March 2009), ISSN 1070-6631.

33. Levi, J.A., Dalley, D.N., \& Aroney, R.S. (1979). Improved combination chemotherapy in advanced gastric-cancer. British Medical Journal, 2, 6203, (December 1979), pp. 1471-1473, ISSN 0959-8138.

34. Liao, C.Y., \& Su, Y.C. (2010). Formation of biodegradable microcapsules utilizing 3D, selectively surface-modified PDMS microfluidic devices. Biomedical Microdevices, 12, 1, (February 2010), pp. 125-133, ISSN: 13872176.

35. Lima, J.L.F.C., Santos, J.L.M., Dias, A.C.B., Ribeiro, M.F.T, \& Zagatto, E.A.G. (2004). Multi-pumping flow systems: an automation tool. Talanta, 64, 5, (December 2004), pp. 1091-1098, ISSN: 0039-9140. 
36. Lin, J.T.Y., \& Yeow, J.T.W. (2007). Enhancing dielectrophoresis effect through novel electrode geometry. Biomedical Microdevices, 9, 6, (December 2007), pp. 823-831, ISSN 1387-2176.

37. Lv, Y.G., Liu, J., Gao, Y.H., \& Xu, B. (2006). Modeling of transdermal drug delivery with a microneedle array. Journal of Micromechanics and Microengineering, 16, 11, (November 2006), pp. 2492-2501, ISSN 0960-1317.

38. Martin-Banderas, L., Flores-Mosquera, M., Riesco-Chueca, P., Rodriguez-Gil, A., Cebolla, A., Chavez, S., \& Ganan-Calvo, A.M. (2005). Flow Focusing: A Versatile Technology to Produce Size- Controlled and Specific-Morphology Microparticles. Small, 1, 7, (July 2005), pp. 688-692. ISSN: 1613-6810.

39. Mejia, A.F., He, P., Luo, D.W., Marquez, M., \& Cheng, Z.D. (2009). Uniform discotic wax particles via electrospray emulsification, Journal of Colloid and Interface Science, 334, 1, (June 2009), pp. 22-28, ISSN: 0021-9797.

40. Mezzenga, R., Schurtenberger, P., Burbidge, A., \& Michel, M. (2005), Understanding foods as soft materials. Nature Materials, 4, 10, (October 2005), pp. 729-740, ISSN: 1476-1122.

41. Migalska, K., Morrow, D.I.J., Garland, M.J., Thakur, R., Woolfson, A.D., \&, Donnelly, R.F. (2011). Laser-engineered dissolving microneedle arrays for transdermal macromolecular drug delivery. Pharmaceutical Research, 28, 8, (August 2011), pp. 1919-1930, ISSN 0724-8741. 
42. Minerick, A.R. (2008). The rapidly growing field of micro and nanotechnology to measure living cells. AIChE Journal, 54, 9, (September 2008), pp. 22302237, ISSN 0001-1541.

43. Moon, S., \& Lee, S.S. (2003). Fabrication of microneedle array using inclined LIGA process. Transducer'03 $12^{\text {th }}$ International Conference on Solid-State Sensors, Actuators and Microsystems, 2, (n.d.), pp. 1546-1549.

44. National Cancer Institute. (n.d.). Stomach Cancer, In: National Institute of Health, September 2010, Available from: $<\mathrm{http}: / /$ www.cancer.gov/cancertopics/types/stomach $>$.

45. Nguyen, N.T., \& Wereley, S.T. (2006). Fundamentals and Applications of Microfluidics ( $2^{\text {nd }}$ Edition), Artech House, ISBN 1580539726, Boston.

46. Pacek, A.W., Chamsart, S., Nienow, A.W., \& Bakker, A. (1999). The influence of impeller type on mean drop size and drop size distribution in an agitated vessel. Chemical Engineering Science 54, 19 (October 1999), pp. 4211-4222, ISSN: 0009-2509.

47. Pai, S.A., Rivankar, S.H., \& Kocharekar, S.S. (2003). Parenteral cisplatin emulsion. United States Patent, US 6,572,884, date of filling (June 14, 2002), date of issue (June 3,2003).

48. Pethig, R. (2010). Dielectrophoresis: status of the theory, technology, and applications. Biomicrofluidics, 4, 3, (September 2010), ISSN 1932-1058. 
49. Power, D.G., Kelsen, D.P., \& Shah, M.A. (2010). Advanced gastric cancer slow but steady progress. Cancer Treatment Reviews, 36, 5, (August 2010), pp. 384-392, ISSN 0305-7372.

50. Rivera, F., Vega-Villegas, M.E., \& Lopez-Brea, M.F. (2007). Chemotherapy of advanced gastric cancer. Cancer Treatment Reviews, 33, 4, (June 2007), pp. 315-324, ISSN 0305-7372.

51. Santini, J.T., Cima, M.J., \& Langer, R.S. (1999). A controlled-release microchip. Nature, 397, 6717, (January 1999), pp. 335-338, ISSN0028-0836.

52. Santini, J.T., Richards, A.C., Scheidt, R.A., Cima, M.J., \& Langer, R.S. (2000). Microchip technology in drug delivery. Annals of Medicine, 32, 6, (September 2000), pp. 377-379, ISSN 0785-3890.

53. Seo, M., Paquet, C., Nie, Z., Xu, S.Q., \& Kumacheva, C. (2007). Microfluidic consecutive flow-focusing droplet generators. Soft Matter, 3, 8, (May 2007), pp. 986-992, ISSN: 1744-683X.

54. Soller, E., Murray, C., Maoddi, P., \& Di Carlo, D. (2011). Rapid prototyping polymers for microfluidic devices and high pressure injections. Lab on a chip, 22, (November 2011), pp. 3752-65, ISSN: 1473-0189.

55. Srivastava, S.K., Gencoglu, A., \& Minerick, A.R. (2011). DC insulator dielectrophoretic applications in microdevice technology: a review. Analytical and Bioanalytical Chemistry, 399, 1, (January 2011), pp. 301-321, ISSN 16182642. 
56. Thiele, J., Steinhauser, D., Pfohl, T., \& Foster, S. (2010). Preparation of monodisperse block copolymer vesicles via flow focusing in microfluidics. Langmuir, 26, 9, (May 2010), pp. 6860-6863, ISSN 0743-7463.

57. Utada. A.S., Lorenceau, E., \& Link, D.R. (2005), Monodisperse double emulsions generated from a microcapillary device. Science, 308, 5721, (April 2005), pp. 537-541, ISSN: 0036-8075

58. Vergauwe, N., Witters, D., Ceyssens, F., Vermeir, S., Verbruggen, B., Puerr, R., \& Lammertyn, J. (2011). A versatile electrowetting-based digital microfluidic platform for quantitative homogeneous and heterogeneous bioassays. Journal of Micromechanics and Microengineering, 21, 5, (May 2011), ISSN 0960-1317.

59. Wang, C., Wang, X., \& Jiang, Z. (2011). Dielectrophoretic driving of blood cells in a microchannel. Biotechnology and Biotechnological Equipment, 25, 2, (May 2011), pp. 2405-2411, ISSN 1310-2818.

60. Wang, P.M., Cornwell, M., Hill, J., \& Prausnitz, M.R. (2006). Precise microinjection into skin using hollow microneedles. Journal of Investigative Dermatology, 126, 5, (May 2006), pp. 1080-1087, ISSN 0022-202X.

61. Weng, C.H., Huang, T., Huang, C., Yeh, C., Lei, H., \& Lee, G. (2011). A suction-type microfluidic immunosensing chip for rapid detection of the dengue virus. Biomedical Microdevices, 13, 3, (June 2011), pp. 585-595, ISSN $1387-2176$. 
62. Wibowo, C., \& Ng, K.M. (2001). Product-Oriented Process Synthesis and Development: Creams and Pastes. AIChE Journal, 47, 12, (December 2001), ISSN: 0001-1541.

63. Xiao, Z.G., \& Young, E.F.Y. (2011). Placement and routing for crossreferencing digital microfluidic biochips. IEEE Transactions on ComputerAided Design of Integrated Circuits and Systems, 30, 7, (July 2011), pp. 10001010, ISSN 0278-0070.

64. Zagnoni, M., Baroud, C.N., \& Cooper, J.M. (2009). Electrically initiated upstream coalescence cascade of droplets in a microfluidic flow. Physical Review E, 80, 4, (October 2009), pp. 046303, ISSN: 1539-3755.

65. Zagnoni, M., Lain, G.L., \& Cooper, J.M. (2010). Electrocoalesence mechanisms of microdroplets using localized electric fields in microfluidic channels. Langmuir, 26, 18, (September 2010), pp. 14443-14449, ISSN 07437463.

66. Zahn, J.D., Deshmukh, A., Pisano, A.P., \& Liepmann, D. (2004). Continuous on-chip micropumping for microneedle enhanced drug delivery. Biomedical Microdevices, 6, 3, (September 2004), pp. 183-190, ISSN 1387-2176.

67. Zhu, H., Mayandad, S., Coskun, A.F., Yagidere, O., \& Ozcan, A. (2011). Optofluidic fluorescent imaging cytometry on a cell phone. Analytical Chemistry, 83, 17, (September 2011), pp. 6641-6647, ISSN 0003-2700. 


\section{Appendix B: Copyright Permission Documentation}

\section{B.1 Figure 2.2 Permission}

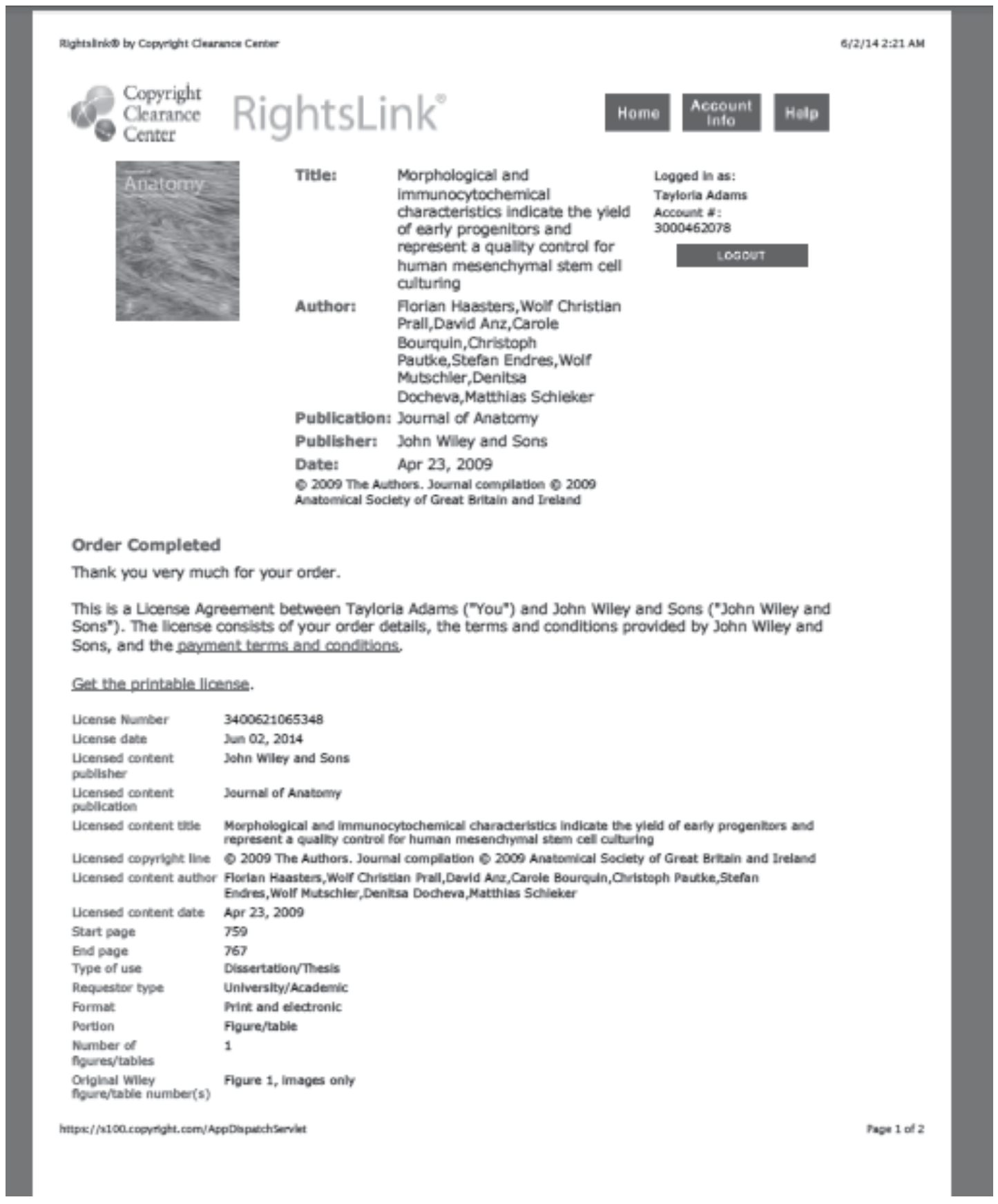




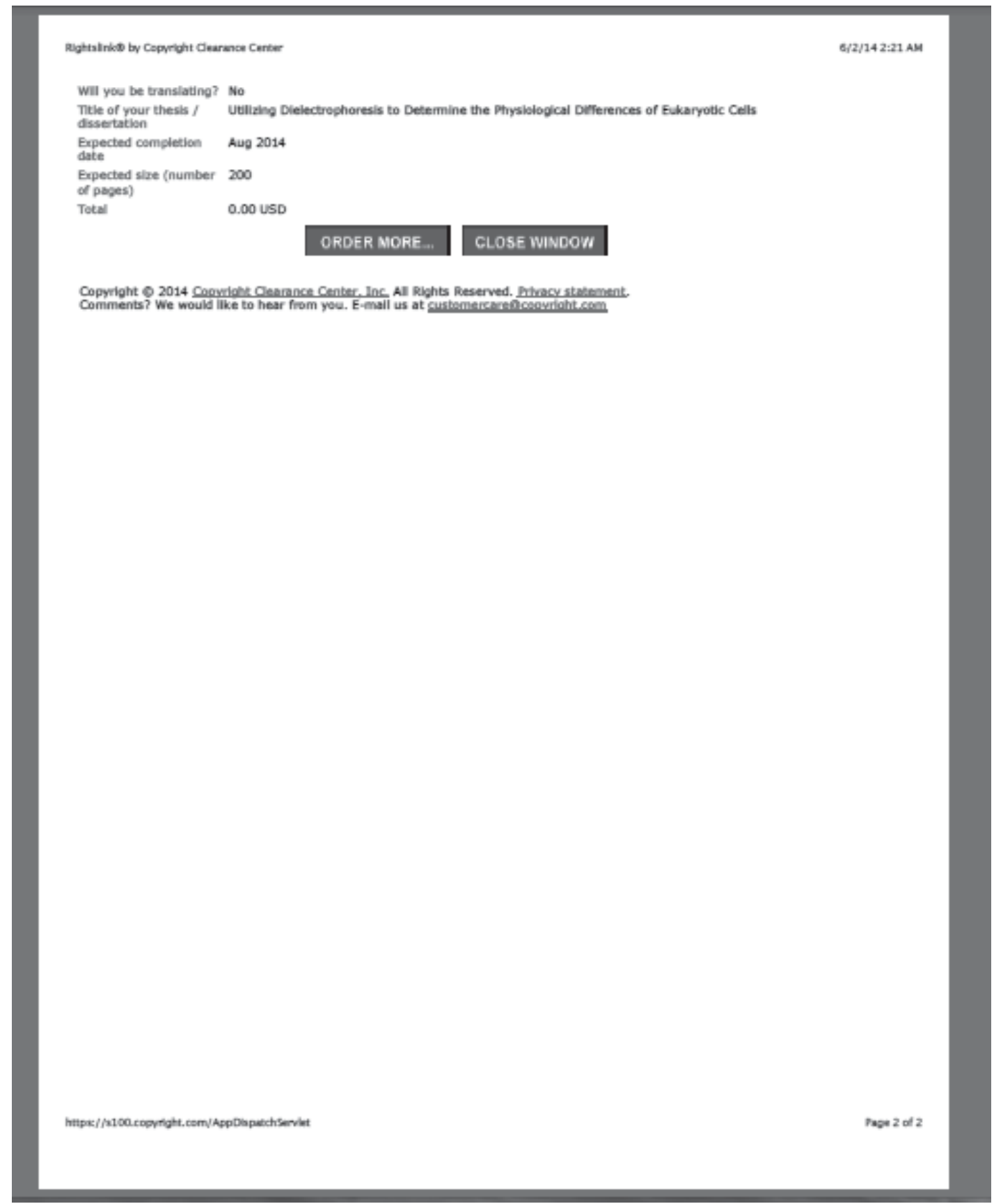




\section{B.2 Figure 2.4a Permission}

Wohtulink Mntate License

$6 / 5 / 241.55$ AN

ELSEVIER UICENSE

TERMS AND CONDITIONS

Jun 05, 2014

This is a License Agreement between Tayloria Adams ("You") and Elsevier ("Elsevier") provided by Copyright Clearance Center ("CCC"). The license consists of your order details, the terms and conditions provided by Elsevier, and the payment terms and conditions.

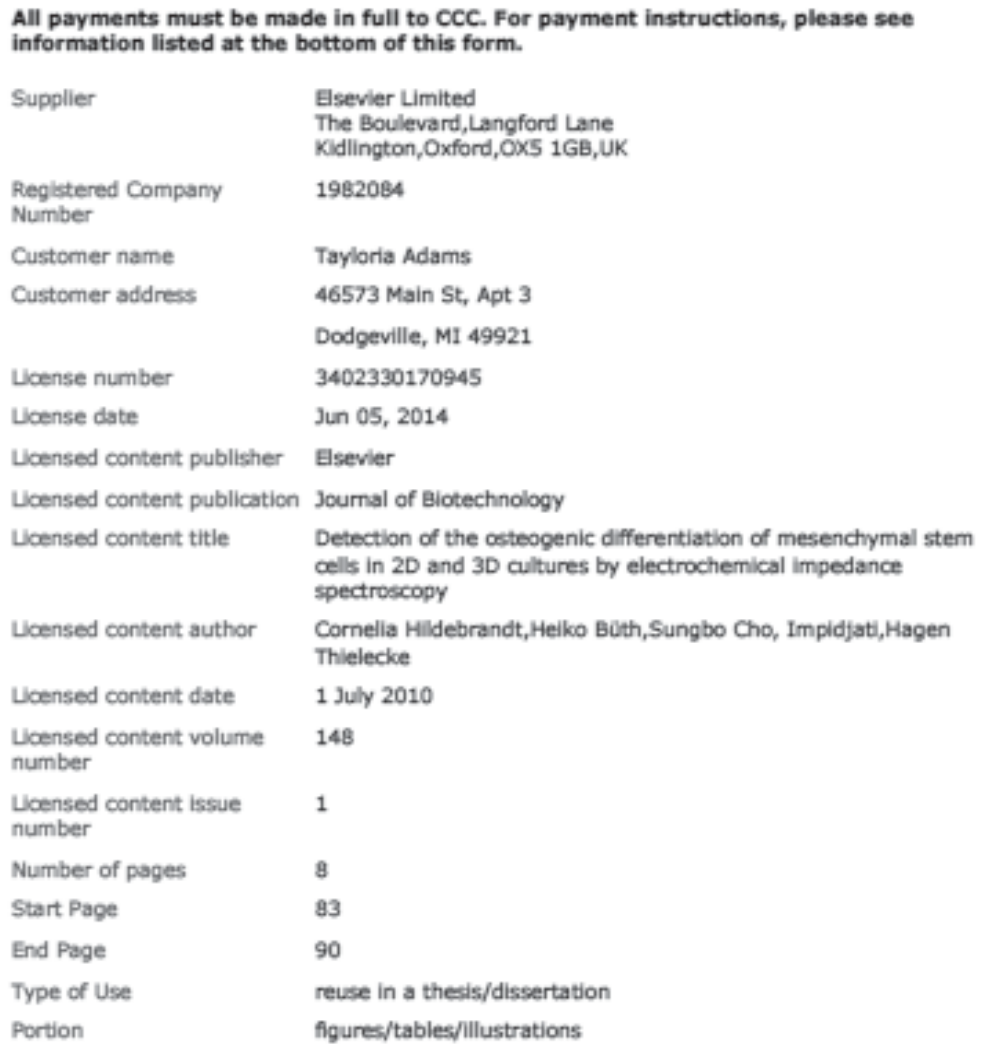

Supplier

Bsevier Limited

The Boulevard, Langford Lane

Kidlington, Oxford,OXS 1GB,UK

Reglatered Company $\quad 1982084$

Number

Tayloria Adams

Customer name

46573 Main St, Apt 3

Dodgeville, MI 49921

Ucense number $\quad 3402330170945$

Ucense date Jun 05, 2014

Uoensed content publisher Esevier

Uoensed content publication Journal of Biotechnology

Ucensed content title

Detection of the osteogenic differentiation of mesenchymal stem cells in 2D and 3D cultures by electrochemical impedance spectroscopy

Lcensed content author Cornelia Hildebrandt,Heiko Buth,Sungbo Cho, Impidjati,Hagen Thielecke

Licensed content date $\quad 1$ July 2010

Loensed content volume 148

number

Loensed content issue $\quad 1$

number

Number of pages 8

Start Page 83

End Page $\quad 90$

Type of Use reuse in a thesis/dissertation

Portion figures/tables/lilustrations

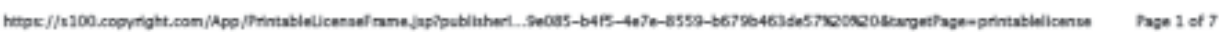




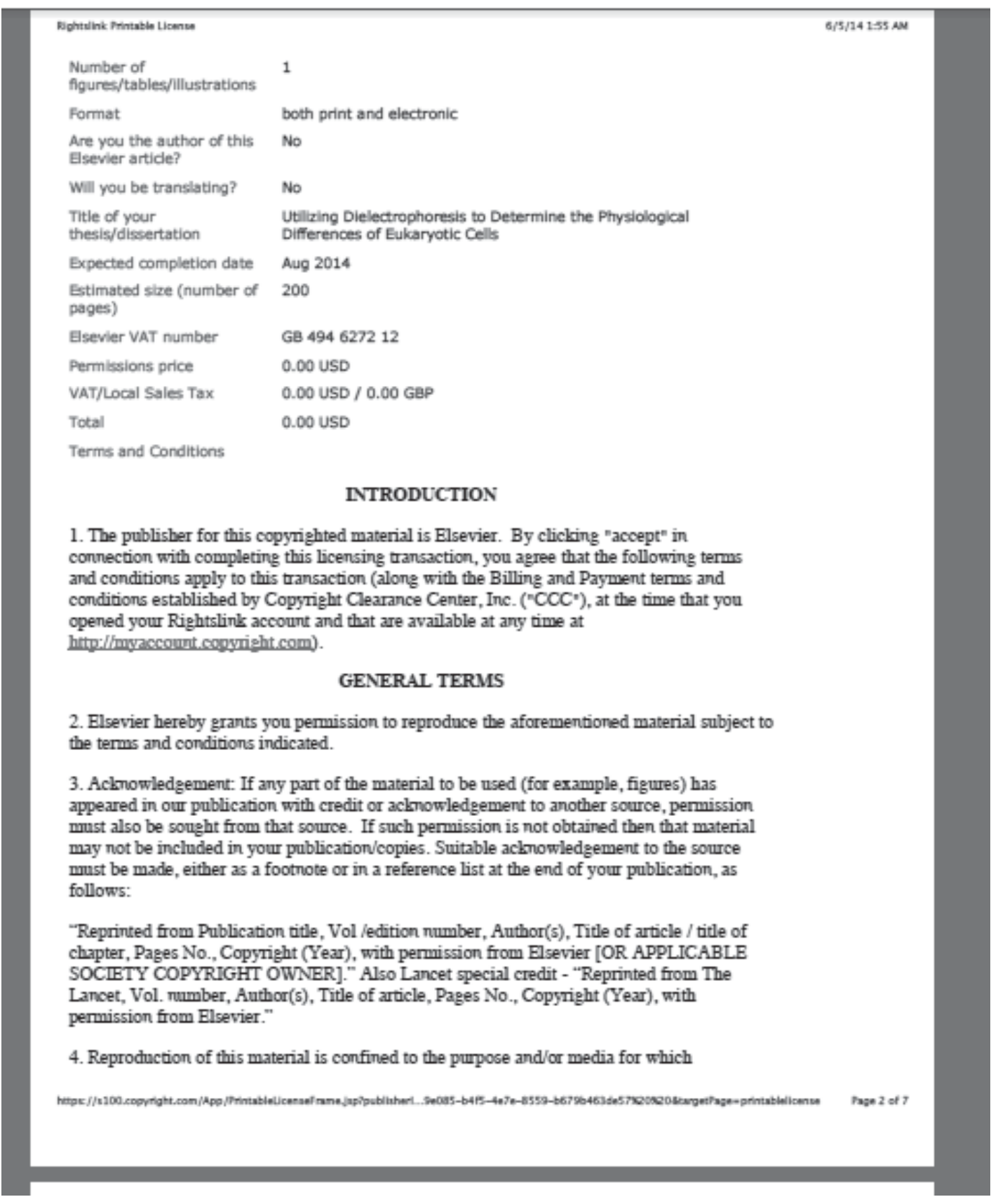


permission is bereby given.

5. Altering/Modifying Material: Not Permitted. However figures and illustrations may be altered/adapted minimally to serve your work. Any other abbreviations, additions, deletions and/or any other alterations shall be made only with prior written authorization of Elsevier Ltd. (Please contact Elsevier at permissions@elsevier.com)

6. If the permission fee for the requested use of our material is waived in this instance, please be advised that your future requests for Elsevier materials may attract a fee.

7. Reservation of Rights: Publisher reserves all rights not specifically granted in the combination of (i) the license details provided by you and accepted in the course of this licensing transaction, (ii) these terms and conditions and (iii) CCCs Billing and Payment terms and conditions.

8. License Contingent Upon Payment: While you may exercise the rights licensed immediately upon issuance of the license at the end of the licensing process for the transaction, provided that you have disclosed complete and accurate details of your proposed use, no license is finally effective unless and until full payment is received from you (either by publisher or by $\mathrm{CCC}$ ) as provided in $\mathrm{CCC}$ s Billing and Payment terms and conditions. If full payment is not received on a timely basis, then any license preliminarily granted shall be deemed automatically revoked and shall be void as if never granted. Further, in the event that you breach any of these terms and conditions or any of CCCs Billing and Payment terms and conditions, the license is automatically revolsed and shall be void as if never granted. Use of materials as described in a revoled license, as well as any use of the materials beyond the scope of an urrevoked license, may constitute copyright infringement and publisher reserves the right to take any and all action to protect its copyright in the materials.

9. Warranties: Publisher makes no representations or warranties with respect to the licensed material.

10. Indemnity: You hereby indemuify and agree to hold harmless publisher and CCC, and their respective officers, directors, employees and agents, from and against any and all claims arising out of your use of the licensed material other than as specifically authorized pursuant to this license.

11. No Transfer of License: This license is personal to you and may not be sublicensed, assigned, or transferred by you to any other person without publisher's written permission.

12. No Amendment Except in Writing: This license may not be amended except in a writing signed by both parties (or, in the case of publisher, by CCC on publisher's behalf).

13. Objection to Contrary Terms: Publisher hereby objects to any terms contained in any purchase order, acknowledgment, check endorsement or other writing prepared by you, which terms are inconsistent with these terms and conditions or CCC's Billing and Payment terms and conditions. These terms and conditions, together with $\mathrm{CCC}^{\prime}$ B Billing and Payment terms and conditions (which are incorporated herein), comprise the entire agreement 
between you and publisber (and $C \mathrm{CC}$ ) concening this licensing transaction. In the event of any conflict between your obligations established by these terms and conditions and those established by $\mathrm{CCC}^{\prime}$ Billing and Payment terms and conditions, these terms and conditions shall control.

14. Revocation: Elsevier or Copyright Clearance Center may deny the permissions described in this License at their sole discretion, for any reason or no reason, with a full refund payable to you. Notice of such denial will be made using the contact information provided by you. Failure to receive such notice will not alter or invalidate the denial. In no event will Elsevier or Copyright Clearance Center be responsible or liable for any costs, expenses or damage incurred by you as a result of a denial of your permission request, other than a refund of the amount(s) paid by you to Elsevier and/or Copyright Clearance Center for denied permissions.

\section{LIMITED LICENSE}

The following terms and conditions apply only to specific license types:

15. Translation: This permission is granted for non-exclusive world English rights only urless your license was granted for translation rights. If you licensed translation rights you may only translate this content into the languages you requested. A professional translator must perform all translations and reproduce the content word for word preserving the integrity of the article. If this license is to re-use 1 or 2 figures then permission is granted for non-exclusive world rights in all languages.

16. Posting licensed content on any Website: The following terms and conditions apply as follows: Licensing material from an Elsevier journal: All content posted to the web site must maintain the copyright information line on the bottom of each image; $A$ hyper-text must be included to the Homepage of the journal from which you are licensing at

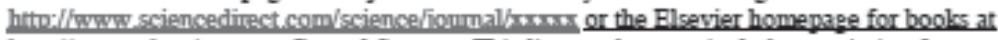
httr://Wwww elsevjer com; Central Storage: This license does not include permission for a scanned version of the material to be stored in a central repository such as that provided by Heron $\sqrt{X}$ anBdu.

Licensing material from an Elsevier book: A hyper-text link must be included to the Elsevier homepage at $\mathrm{htt}$ : $/ / \mathrm{w} w \mathrm{ww}$ elsevier com . All content posted to the web site must maintain the copyright information line on the bottom of each image.

Posting licensed content on Electronic reserve: In addition to the above the following clauses are applicable: The web site must be password-protected and made available only to bona fide students registered on a relevant course. This permission is granted for 1 year only. You may obtain a new license for future website posting.

For journal authors: the following clauses are applicable in addition to the above: Permission granted is limited to the author accepted manuscript version* ${ }^{*}$ of your paper.

*Accepted Author Manuscript (AAM) Definition: An accepted author manuscript (A.AM) 
is the author's version of the manuscript of an article that has been accepted for publication and which may include any author-incorporated changes suggested through the processes of submission processing, peer review, and editor-author commmications. A.AMs do not include other publisher value-added contributions such as copy-editing, formatting, technical enhancements and (if relevant) pagination.

You are not allowed to download and post the published joumal article (whether PDF or HTML, proof or final version), nor may you scan the printed edition to create an electronic version. A hyper-text must be included to the Homepage of the journal from which you are licensing at http://www sciencedirect.com/science/joumal/swr.x. As part of our normal production process, you will receive an e-mail notice when your article appears on Elsevier's online service ScienceDirect (www sciencedirect com). That e-mail will include the article's Digital Object Identifier (DOI). This number provides the electronic link to the published article and should be included in the posting of your personal version. We ask that you wait until you receive this e-mail and have the DOI to do any posting.

Posting to a repository: Authors may post their AAM immediately to their employer's institutional repository for internal use only and may malse their manuscript publically available after the journal-specific embargo period has ended.

Please also refer to Elsevier's Anticle Postine Policy for further information.

18. For book autbors the following clauses are applicable in addition to the above: Authors are permitted to place a brief summary of their work online only.. You are not allowed to download and post the published electronic version of your chapter, nor may you scan the printed edition to create an electronic version. Posting to a repository: Authors are permitted to post a summary of their chapter only in their institution"s repository.

20. Thesis/Dissertation: If your license is for use in a thesis/dissertation your thesis may be submitted to your institution in either print or electronic form. Should your thesis be published commercially, please reapply for permission. These requirements include permission for the Library and Archives of Canada to supply single copies, on demand, of the complete thesis and include permission for UMI to supply single copies, on demand, of the complete thesis. Should your thesis be published commercially, please reapply for permission.

\section{Elsevier Open Access Terms and Conditions}

Elsevier publisbes Open Access articles in both its Open Access journals and via its Open Access articles option in subscription journals.

Authors publishing in an Open Access joumal or who choose to make their article Open Access in an Elsevier subscription jourul select one of the following Creative Commons user licenses, which define how a reader may reuse their work: Creative Commons Attribution License (CC BY), Creative Commons Attribution - Non Commercial ShareAlike (CC BY NC SA) and Creative Commons Attubution - Non Commercial - No Derivatives (CC BY NC ND) 
Terms \& Conditions applicable to all Elsevier Open Access articles:

Any reuse of the article must not represent the author as endorsing the adaptation of the article nor should the article be modified in such a way as to damage the author's honour or reputation.

The author(s) must be appropriately credited.

If any part of the material to be used (for example, figures) has appeared in our publication with credit or acknowledgement to another source it is the responsibility of the user to ensure their reuse complies with the terms and conditions determined by the rights holder.

\section{Additional Terms \& Conditions applicable to each Creative Commons user license:}

CC BY: You may distribute and copy the article, create extracts, abstracts, and other revised versions, adaptations or derivative works of or from an article (such as a translation), to include in a collective work (such as an anthology), to text or data mine the article, including for commercial purposes without permission from Elsevier

CC BY NC SA: For non-commercial purposes you may distribute and copy the article, create extracts, abstracts and other revised versions, adaptations or derivative works of or from an article (such as a translation), to include in a collective work (such as an anthology), to text and data mine the article and license new adaptations or creations under identical terms without permission from Elsevier

CC BY NC ND: For non-commercial purposes you may distribute and copy the article and include it in a collective work (such as an anthology), provided you do not alter or modify the article, without permission from Elsevier

Any commercial reuse of Open Access articles published with a CC BY NC SA or CC BY NC ND license requires permission from Elsevier and will be subject to a fee.

Commercial reuse includes:

- Promotional pupposes (advertising or marketing)

- Commercial exploitation (e.g- a product for sale or loan)

- Systematic distribution (for a fee or free of charge)

Please refer to Elsevier's Open. Access Policy for further information.

\section{Other Conditions:}

v1.7 
If you would like to pay for this license now, please remit this license along with your payment made payable to "COPYRIGHT CLEARANCE CENTER" otherwise you will be invoiced within $\mathbf{4 8}$ hours of the license date. Payment should be in the form of a check or money order referencing your account number and this involice number 501320378 . Once you receive your invoice for this order, you may pay your invoice by credit card.

Please follow instructions provided at that time.

Make Payment To:

Copyright Clearance Center

Dept 001

P.0. Box 843006

Boston, MA 02284-3006

For suggestions or comments regarding this order, contact RightsLink Customer Support: customercare ecomriohit.com or +1-877-622-5543 (toll free in the US) or +1 978-646-2777.

Gratis licenses (referencing $\$ 0$ in the Total field) are free. Please retain this printable license for your reference. No payment is required. 


\section{B.3 Figure 2.4b Permission}

Wohtsink Mrntable Liense

$6 / 5 / 24200$ AN

ELSEVIER UICENSE

TERMS AND CONDITIONS

Jun 05, 2014

This is a License Agreement between Tayloria Adams ("You") anti Elsevier ("Elsevier")

provided by Copyright Clearance Center ("CCC"). The license consists of your order details, the terms and conditions provided by Blsevier, and the payment terms and conditions.

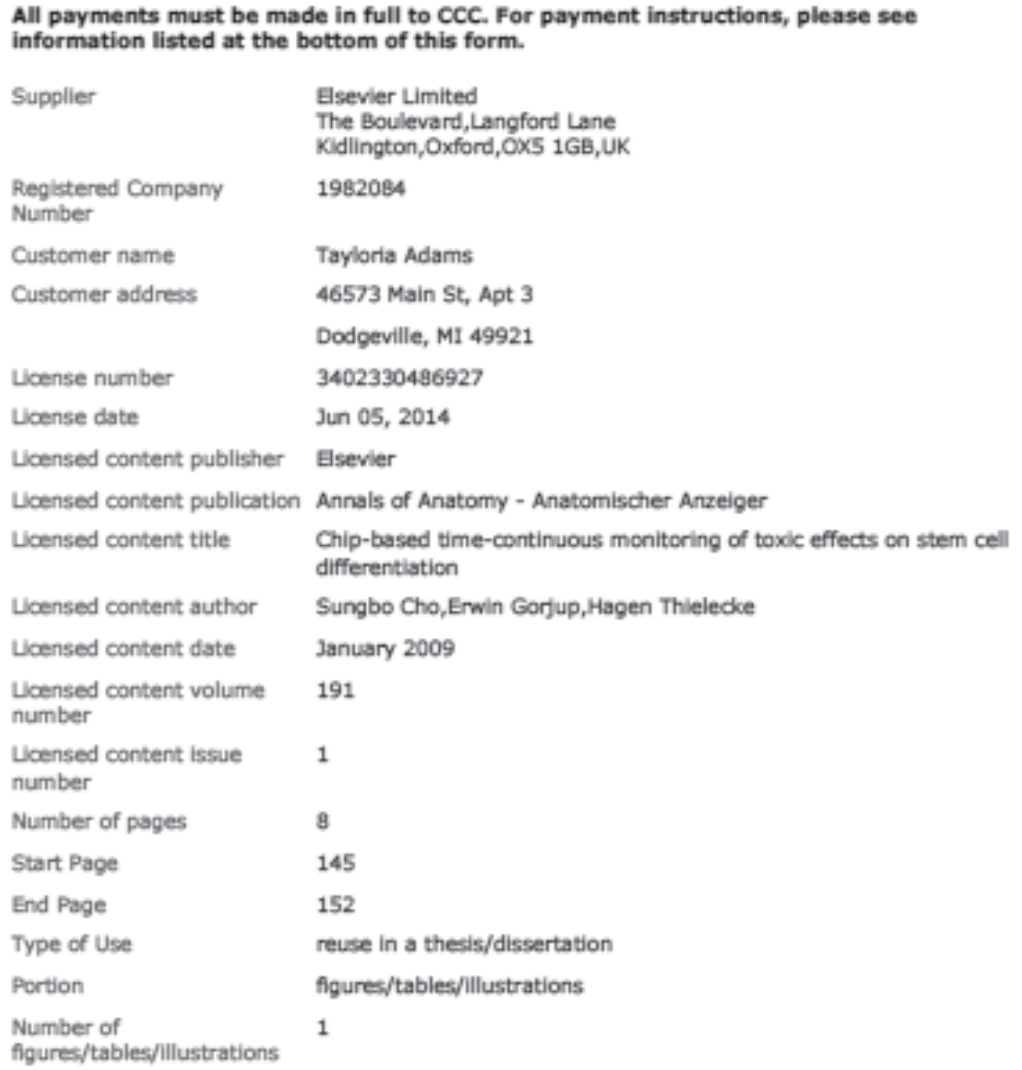


Format

Are you the author of this

Esevier artide?

Will you be translating?

Title of your

thesis/dissertation

Expected completion date

Estimated size (number of pages)

Elsevier VAT number

Permissions price

VAT/Local Sales Tax

Total

Terms and Conditions both print and electronic

No

No

Utllzing Dielectrophoresis to Determine the Physiological Differences of Eukaryotic Cells

Aug 2014

200

GB 494627212

0.00 USD

0.00 USD / $0.00 \mathrm{GeP}$

0.00 USD

\section{INTRODUCTION}

1. The publisber for this copyrighted material is Elsevier. By clicking "accept" in connection with completing this licensing transaction, you agree that the following terms and conditions apply to this transaction (along with the Billing and Payment terms and conditions established by Copyright Clearance Center, Inc. ("CCC"), at the time that you opened your Rightslink account and that are available at any time at htro://mvaccount coovright. com).

\section{GENERAL TERMS}

2. Elsevier hereby grants you permission to reproduce the aforementioned material subject to the terms and conditions indicated.

3. Acknowledgement: If any part of the material to be used (for example, figures) has appeared in our publication with credit or acknowledgement to another source, permission must also be sought from that source. If such permission is not obtained then that material may not be included in your publication/copies. Suitable acknowledgement to the source must be made, either as a footnote or in a reference list at the end of your publication, as follows:

"Reprinted from Publication title, Vol /edition number, Author(s), Title of article / title of chapter, Pages No., Copyright (Year), with permission from Elsevier [OR APPLICABLE SOCIETY COPYRIGHT OWNER]." Also Lancet special credit - "Reprinted from The Lancet, Vol. number, Author(s), Title of article, Pages No., Copyright (Year), with permission from Elsevier."

4. Reproduction of this material is confined to the purpose and/or media for which permission is bereby given. 
5. Altering/Modifying Material: Not Permitted. However figures and illustrations may be altered/adapted minimally to serve your work. Any other abbreviations, additions, deletions and/or any other alterations shall be made only with prior written authorization of Elsevier Ltd. (Please contact Blsevier at permissions@elsevier.com)

6. If the permission fee for the requested use of our material is waived in this instance, please be advised that your future requests for Blsevier materials may attract a fee.

7. Reservation of Rights: Publisher reserves all rights not specifically granted in the combination of (i) the license details provided by you and accepted in the course of this licensing transaction, (ii) these terms and conditions and (iii) CCC's Billing and Payment terms and conditions.

8. License Contingent Upon Payment. While you may exercise the rights licensed immediately upon issuance of the license at the end of the licensing process for the transaction, provided that you have disclosed complete and accurate details of your proposed use, no license is firally effective unless and until full payment is received from you (either by publisher or by $\mathrm{COC}$ ) as provided in $\mathrm{CCC}$ s Billing and Payment terms and conditions. If full payment is not received on a timely basis, then any license preliminarily granted shall be deemed automatically revoked and shall be void as if never granted. Further, in the event that you breach any of these terms and conditions or any of CCCs Billing and Payment terms and conditions, the license is automatically revolked and shall be void as if never granted. Use of materials as described in a revoked license, as well as any use of the materials beyond the scope of an urrevoked license, may constitute copyright infringement and publisher reserves the right to take any and all action to protect its copyright in the materials.

9. Warranties: Publisher makes no representations or warranties with respect to the licensed material.

10. Indemnity. You hereby indemnify and agree to hold harnless publisher and $C C C$, and their respective officers, directors, employees and agents, from and against any and all claims arising out of your use of the licensed material other than as specifically authorized pursuant to this license.

11. No Transfer of License: This license is personal to you and may not be sublicensed, assigned, or transferred by you to any other person without publisher's written permission.

12. No Amendment Except in Writing: This license may not be amended except in a writing signed by both parties (or, in the case of publisher, by CCC on publisher's behalf).

13. Objection to Contrary Terms: Publisber bereby objects to any terms contained in any purchase order, acknowledement, check endorsement or other writing prepared by you, which terms are inconsistent with these terms and conditions or CCC's Billing and Payment terms and conditions. These terms and conditions, together with $\mathrm{CCC}$ 's Billing and Payment terms and conditions (which are incorporated herein), comprise the entire agreement between you and publisher (and CCC) concening this licensing transaction. In the event of any conflict between your obligations established by these terms and conditions and those 
established by CCC's Billing and Payment terms and conditions, these terms and conditions shall control.

14. Revocation: Elsevier or Copyright Clearance Center may deny the permissions described in this License at their sole discretion, for any reason or no reason, with a full refurd payable to you. Notice of such denial will be made using the contact information provided by you. Failure to receive such notice will not alter or invalidate the denial. In no event will Elsevier or Copyright Clearance Center be responsible or liable for any costs, expenses or damage incurred by you as a result of a denial of your permission request, other than a refund of the amount(s) paid by you to Elsevier and/or Copyright Clearance Center for denied permissions.

\section{LIMTTED LICENSE}

The following terms and conditions apply only to specific license types:

15. Translation: This permission is granted for non-exclusive world English rights only ulless your license was granted for translation rights. If you licensed translation rights you may only translate this content into the languages you requested. A professional translator must perform all translations and reproduce the content word for word preserving the integrity of the article. If this license is to re-use 1 or 2 figures then permission is granted for non exclusive world rights in all languages.

16. Posting licensed content on any Website: The following terms and conditions apply as follows: Licensing material from an Elsevier jourual: All content posted to the web site must maintain the copyright information line on the bottom of each image; $A$ hyper-text must be included to the Homepage of the journal from which you are licensing at

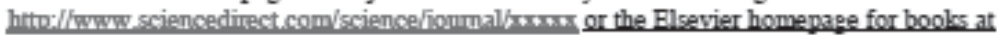
httr://Wwwwelsevjer.com; Central Storage: This license does not include permission for a scanned version of the material to be stored in a central repository such as that provided by Heron $\mathrm{X}$ anEdn.

Licensing material from an Blsevier book: A hyper-text link must be included to the Elsevier homepage at httip://www elsevier com. All content posted to the web site must maintain the copyright information line on the bottom of each image.

Posting licensed content on Electronic reserve: In addition to the above the following clauses are applicable: The web site must be password-protected and made available only to bona fide students registered on a relevant course. This permission is granted for 1 year only. You may obtain a new license for future website posting.

For journal authors: the following clauses are applicable in addition to the above: Permission granted is limited to the author accepted manuscript version ${ }^{*}$ of your paper.

*Accepted Author Manuscript (AAM) Definition: An accepted author manuscript (A.AM) is the author's version of the manuscript of an article that has been accepted for publication and which may include any author-incorporated changes suggested through the processes of submission processing, peer review, and editor-author commmications. A.AMs do not 
include other publisher value-added contributions such as copy-editing, formatting, technical enhancements and (if relevant) pagination.

You are not allowed to download and post the published journal article (whether PDF or HTML, proof or final version), nor may you scan the printed edition to create an electronic version. A hyper-text must be included to the Homepage of the journal from which you are

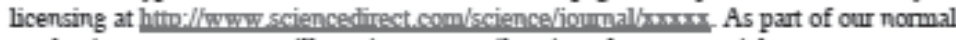
production process, you will receive an e-mail notice when your article appears on Elsevier's online service ScienceDirect (www.sciencedirect.com). That e-mail will include the article's Digital Object Identifier (DOI). This number provides the electronic link to the published article and should be included in the posting of your personal version. We ask that you wait until you receive this e-mail and have the DOI to do any posting.

Posting to a repository: Authors may post their A.AM immediately to their employer's instimutional repository for internal use only and may malse their manuscript publically available after the journal-specific embargo period has ended.

Please also refer to Blsevier's Anticle-Postine Policy for further information.

18. For book authors the following clauses are applicable in addition to the above: Authors are permitted to place a brief summary of their work online only.. You are not allowed to download and post the published electronic version of your chapter, nor may you scan the printed edition to create an electronic version. Posting to a repository: Authors are permitted to post a summary of their chapter only in their institution's repository.

20. Thesis/Dissertation: If your license is for use in a thesis/dissertation your thesis may be submitted to your institution in either print or electronic form. Should your thesis be published commercially, please reapply for permission. These requirements include permission for the Library and Archives of Canada to supply single copies, on demand, of the complete thesis and include permission for UMI to supply single copies, on demand, of the complete thesis. Should your thesis be published commercially, please reapply for permission.

\section{Elsevier Open Access Terms and Conditions}

Elsevier publisbes Open Access articles in both its Open Access journals and via its Open Access articles option in subscription joumals.

Authors publishing in an Open Access joumal or who choose to make their article Open Access in an Elsevier subscription journal select one of the following Creative Commons user licenses, which define how a reader may reuse their work: Creative Commons Attribution License (CC BY), Creative Commons Attribution - Non Commercial ShareAlike (CC BY NC SA) and Creative Commons Attribution - Non Commercial - No Derivatives (CC BY NC ND)

Terms \& Conditions applicable to all Elsevier Open Access articles: 
Any reuse of the article must not represent the author as endorsing the adaptation of the article nor should the article be modified in such a way as to damage the author's honour or reputation.

The author(s) must be appropriately credited.

If any part of the material to be used (for example, figures) has appeared in our publication with credit or acknowledgement to another source it is the responsibility of the user to ensure their reuse complies with the terms and conditions determined by the rights holder.

\section{Additional Terms \& Conditions applicable to each Creative Commons user license:}

CC BY: You may distribute and copy the article, create extracts, abstracts, and other revised versions, adaptations or derivative works of or from an article (such as a translation), to include in a collective work (such as an anthology), to text or data mine the article, including for commercial purposes without permission from Elsevier

CC BY NC SA: For non-commercial pupposes you may distribute and copy the article, create extracts, abstracts and other revised versions, adaptations or derivative works of or from an article (such as a translation), to include in a collective work (such as an anthology), to text and data mine the article and license new adaptations or creations under identical terms without permission from Elsevier

CC BY NC ND: For non-commercial purposes you may distribute and copy the article and include it in a collective work (such as an anthology), provided you do not alter or modify the article, without permission from Elsevier

Any commercial reuse of Open Access articles published with a CC BY NC SA or CC BY NC ND license requires permission from Elsevier and will be subject to a fee.

Commercial reuse includes:

- Promotional purposes (advertising or marketing)

- Commercial exploitation (e.g- a product for sale or loan)

- Systematic distribution (for a fee or free of charge)

Please refer to Elceviers Open. Access. Policy for further information.

\section{Other Conditions:}

$\mathrm{vl} .7$

If you would like to pay for this license now, please remit this license along with your 
payment made payable to "COPYRIGHT CLEARANCE CENTER" otherwise you will be

invoiced within $\mathbf{4 8}$ hours of the license date. Payment should be in the form of a check or money order referencing your account number and this involce number 501320381. Once you receive your invoice for this order, you may pay your invoice by credit card. Please follow instructions provided at that time.

Make Payment To:

Copyright Clearance Center

Dept 001

P.O. Box 843006

Boston, MA 02284-3006

For suggestions or comments regarding this order, contact RightsLink Customer

Support: customercarefecomrioht.com or $+1-877-622-5543$ (toll free in the US) or +1 978-646-2777.

Gratis licenses (referencing $\$ 0$ in the Total field) are free. Please retain this printable license for your reference. No payment is required. 


\section{B.4 Figure 2.7 Permission}

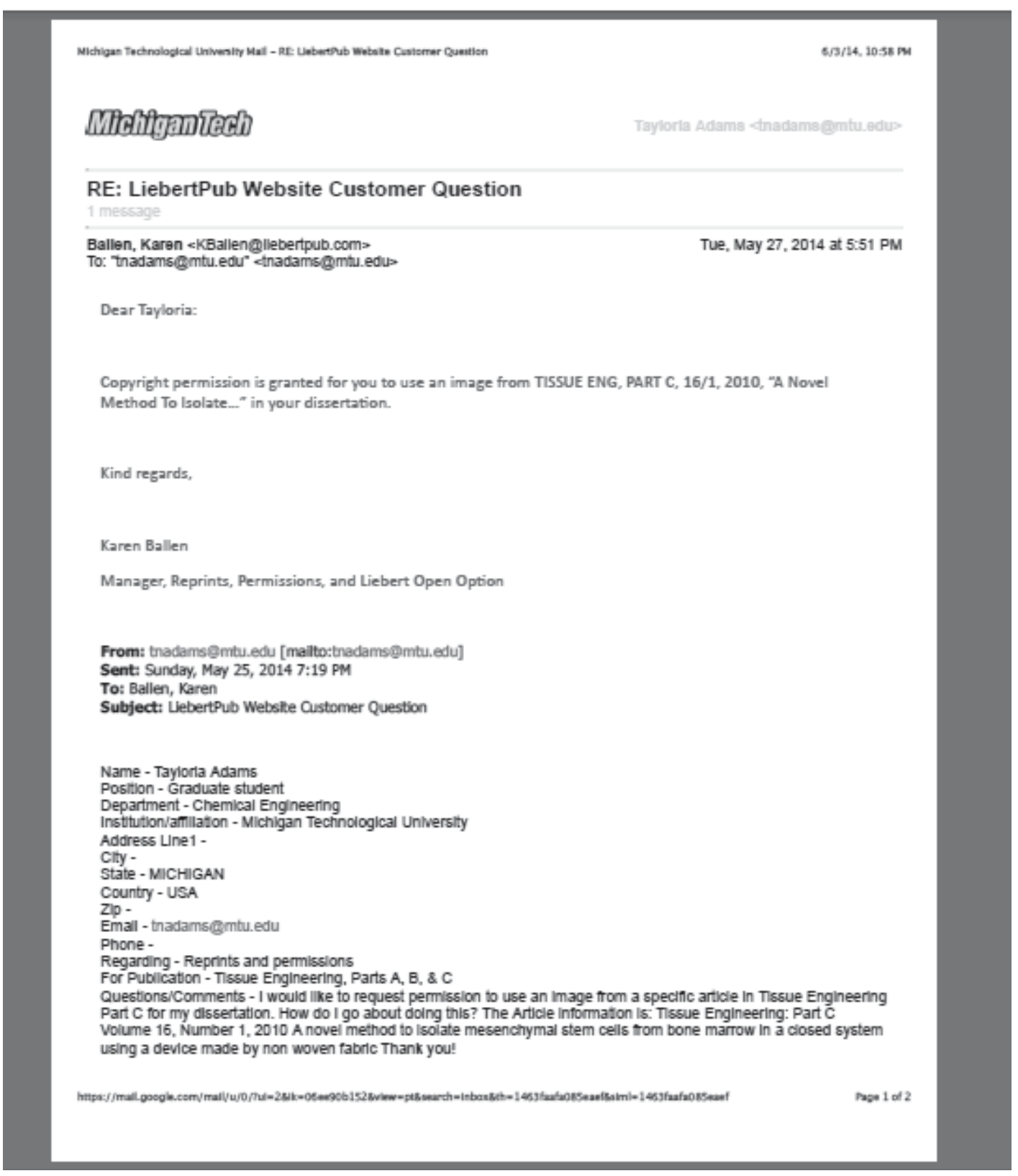




\title{
B.5 Figure 3.7a Permission
}

Reghtsink Mintuble Lemse

SPRINGER LICENSE

TERMS AND CONDITIONS

Jun 09, 2014

This is a License Agreement between Tayloria Adams ("You") and Springer ("Springer") provided by Copyright Clearance Center ("CCC"). The license consists of your order details, the terms and conditions provided by Springer, and the payment tems and conditions.

\author{
All payments must be made in full to $C C C$. For payment instructions, please see \\ information listed at the bottom of this form. \\ License Number $\quad 3404600131894$ \\ License date Jun 09, 2014 \\ Licensed content publisher Springer \\ Licensed content publication Analytical and Bioanalytical Chemistry \\ Licensed content title Selective trapping of single mammallan breast cancer cells by \\ insulator-based dielectrophoresis \\ Ucensed content author Sanchari Bhattacharya \\ Licensed content date Jan 1, 2014 \\ Volume number $\quad 406$ \\ Issue number 7 \\ Type of Use Thesis/Dissertation \\ Portion Figures \\ Author of this Springer No \\ article \\ Order reference number None \\ Original figure numbers Figure $1 \mathrm{a}$ \\ Thtle of your thesis / Utilizing Dielectrophoresis to Determine the Physiological \\ dissertation \\ Differences of Eukaryotic Cells \\ Expected completion date Aug 2014 \\ Estimated size(pages) $\quad 200$ \\ Total $\quad 0.00$ USD \\ Terms and Conditions \\ Introduction \\ The publisher for this copyrighted material is Springer Science + Business Media. By \\ clicking "accept" in connection with completing this licensing transaction, you agree that the
}

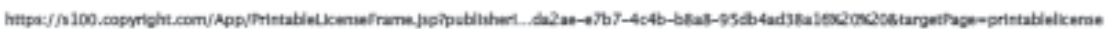

$\operatorname{mop} 1$ of 4 
following terms and conditions apply to this transaction (along with the Billing and Payment terms and conditions establisbed by Copyright Clearance Center, Inc. (" $\mathrm{CCC}$ "), at the time that you opened your Rightslink account and that are available at any time at

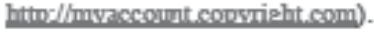

\section{Limited License}

With reference to your request to reprint in your thesis material on which Springer Science and Business Media control the copyright, permission is granted, free of charge, for the use indicated in your enquiry.

Licenses are for one-time use only with a maximum distribution equal to the number that you identified in the licensing process.

This License includes use in an electronic form, provided its password protected or on the university's intranet or repository, including UMI (according to the definition at the Sherpa website: http://www sherpa.ac.uk/romeo/). For any other electronic use, please contact Springer at (permissions.dordrecht $₫$ springer.com or permissions heidelberg $@$ springer.com).

The material can only be used for the purpose of defending your thesis limited to uriversityuse only. If the thesis is going to be publisbed, permission needs to be re-obtained (selecting "book/textbook" as the type of use).

Although Springer holds copyright to the material and is entitled to negotiate on rights, this license is only valid, subject to a courtesy information to the author (address is given with the article/chapter) and provided it concerns original material which does not canry references to other sources (if material in question appears with credit to another source, authorization from that source is required as well).

Permission free of charge on this occasion does not prejudice any rights we might have to charge for reproduction of our copyrighted material in the future.

Altering/Modifying Material: Not Peruitted

You may not alter or modify the material in any manner. Abbreviations, additions, deletions and/or any other alterations shall be made only with prior written authorization of the author(s) and/or Springer Science + Business Media. (Please contact Springer at (permissions.dordrecht@springer.com or permissions.heidelberg@springer.com)

\section{Reservation of Rights}

Springer Science + Business Media reserves all rights not specifically granted in the combination of (i) the license details provided by you and accepted in the course of this licensing transaction, (ii) these terms and conditions and (iii) COCs Billing and Payment terms and conditions.

Copyright Notice:Disclaimer

You must include the following copyright and permission notice in connection with any reproduction of the licensed material: "Springer and the original publisher/journal title, volume, year of publication, page, chapter/article title, name(s) of author(s), figure number(s), original copyright notice) is given to the publication in which the material was 
originally published, by adding; with kind permission from Springer Science and Business Media"

Wananties: None

Example 1: Springer Science + Business Media makes no representations or warranties with respect to the licensed material.

Bxample 2: Springer Science + Business Media makes no representations or wanranties with respect to the licensed material and adopts on its own behalf the limitations and disclaimers established by CCC on its behalf in its Billing and Payment terms and conditions for this licensing transaction.

\section{Indemuity}

You here by indemnify and agree to hold harmless Springer Science + Business Media and $\triangle C C$, and their respective officers, directors, employees and agents, from and against any and all claims arising out of your use of the licensed material other than as specifically authorized pursuant to this license.

No Transfer of License

This license is personal to you and may not be sublicensed, assigned, or transferred by you to any other person without Springer Science + Business Media's written permission.

No Amendment Bxcept in Writing

This license may not be amended except in a writing signed by both parties (or, in the case of Springer Science + Business Media, by CCC on Springer Science + Business Media's behalf).

\section{Objection to Contrary Terms}

Springer Science + Business Media hereby objects to any terms contained in any purchase order, acknowledgment, check endorsement or other writing prepared by you, which terms are inconsistent with these terms and conditions or $\mathrm{CCC}$ 's Billing and Payment terms and conditions. These terms and conditions, together with $\mathrm{CCC}^{\prime}$ s Billing and Payment terms and conditions (which are incorporated berein), comprise the entire agreement between you and Springer Science + Business Media (and CCC) concerning this licensing transaction. In the event of any conflict between your obligations established by these terms and conditions and those establisbed by $\mathrm{CCC}^{\prime}$ Billing and Payment terms and conditions, these terms and conditions shall control.

Jurisdiction

All disputes that may arise in connection with this present License, or the breach thereof, shall be settled exclusively by arbitration, to be beld in The Netherlands, in accordance with Dutch law, and to be conducted under the Rules of the 'Netherlands Arbitrage Instinut (Netherlands Institute of Arbitration) $O R$ :

All disputes that may arise in connection with this present License, or the breach thereof, shall be setfled exclnsively by arbitration, to be held in the Federal Republic of Germany, in accordance with German law. 
Pughtalink Printuble Lemis

Other terms and conditions:

v1.3

If you would like to pay for this license now, please remit this license along with your payment made payable to "COPYRIGHT CLEARANCE CENTER" otherwise you will be

invoiced within $\mathbf{4 8}$ hours of the license date. Payment should be in the form of a check or money order referencing your account number and this invoice number 501323074.

Once you recelve your involce for this order, you may pay your invoice by credit card.

Please follow instructions provided at that time.

Make Payment To:

Copyright Clearance Center

Dept 001

P.O. Box 843006

Boston, MA 02284-3006

For suggestions or comments regarding this order, contact RightsLink Customer Supporti customercarefcopyilght.com or $+1-877-622-5543$ (toll free in the US) or +1 978-646-2777.

Gratis licenses (referencing $\mathbf{\$ 0}$ in the Total field) are free. Please retain this printable license for your reference. No payment is required. 


\section{B.6 Figure 3.7b Permission}

Roghtilink Mintuble Ucente

SMP14, 1:22AM

\section{JOHN WILEY AND SONS LICENSE}

TERMS AND CONDITIONS

Jun 09,2014

This is a License Agreement between Tayloria Adams ("You") and John Wiley and Sons ("John Wiley and Sons") provided by Copyright Clearance Center ("CCC"). The license consists of your order details, the terms and conditions provided by John Wiley and Sons, and the payment terms and conditions.

All payments must be made in full to CCC. For payment instructions, please see information listed at the bottom of this form.

License Number

3404600697169

Ucense date

Jun 09,2014

Licensed content publisher

John Wiley and Sons

Licensed content publication Electrophoresis

Licensed content title

Silicon insulator-based dielectrophoresis devices for minimized heating effects

Licensed copyright line

9 2012 WILYY-VCH Verlag GmbH \& Co. KGaA, Weinheim

Licensed content author

Phillip Zellner,Masoud Agah

Licensed content date

Aug 17, 2012

Start page

2498

End page

2507

Type of use

Dissertation/Thesis

Requestor type

University/Academic

Format

Print and electronic

Portion

Figure/table

Number of figures/tables

Original Wiley figure/table number(s)

Will you be translating?

Titie of your thesis /

dissertation

1

Figure 2, B1 and B2

No

Utilizing Dielectrophoresis to Determine the Physiological Differences of Eukaryotic Cells

Expected completion date

Aug 2014

Expected size (number of 200 pages)

Total

0.00 USD 
Terms and Conditions

\section{TERMS AND CONDITIONS}

This copyrighted material is owned by or exclusively licensed to Jobn Wiley \& Sons, Inc. or one of its group comparies (each a"Wiley Company") or handled on behalf of a society with which a Wiley Company has exclusive publishing rights in relation to a particular work (collectively "WILEY"). By clicking 0 accept $\hat{\theta}$ in connection with completing this licensing transaction, you agree that the following terms and conditions apply to this transaction (along with the billing and payment terms and conditions established by the Copyright Clearance Center Inc., ("CCC's Billing and Payment terms and conditions"), at the time that you opened your Rightslink account (these are available at any time at http://myaccourt.copyripht.com).

\section{Terms and Conditions}

- The materials you have requested permission to reproduce or reuse (the "Wiley Materials") are protected by copyright.

- You are bereby granted a personal, non-exclusive, non-sub licensable (on a standalone basis), non-transferable, worldwide, limited license to reproduce the Wiley Materials for the purpose specified in the licensing process. This license is for a onetime use only and limited to any maximum distribution number specified in the license. The first instance of republication or reuse granted by this licence must be completed within two years of the date of the grant of this licence (although copies prepared before the end date may be distributed thereafter). The Wiley Materials shall not be used in any other manner or for any other purpose, beyond what is granted in the license. Permission is granted subject to an appropriate ackrowledgement given to the author, title of the material/book/journal and the publisher. You shall also duplicate the copyright notice that appears in the Wiley publication in your use of the Wiley Material. Permission is also granted on the understanding that nowhere in the text is a previously published source acknowledged for all or part of this Wley Material. Any third party content is expressly excluded from this permission.

- With respect to the Wiley Materials, all rights are reserved. Except as expressly granted by the terms of the license, no part of the Wiley Materials may be copied, modified, adapted (except for minor reformatting required by the new Publication), translated, reproduced, transferred or distributed, in any form or by any means, and no derivative works may be made based on the Wiley Materials without the prior permission of the respective copyright owner. You may not alter, remove or suppress in any manner any copyright, trademark or other notices displayed by the Wiley Materials. You may not license, rent, sell, loan, lease, pledge, offer as security, transfer or assign the Wiley Materials on a stand-alone basis, or any of the rights granted to you bereunder to any other person.

- The Wiley Materials and all of the intellectual property rights therein shall at all times 
remain the exclusive property of John Wiley \& Sons Inc, the Wiley Companies, or their respective licensors, and your interest therein is only that of having possession of and the right to reproduce the Wiley Materials pursuant to Section 2 herein during the continuance of this Agreement. You agree that you own no right, title or interest in or to the Wiley Materials or any of the intellectual property rights therein. You shall have no rights hereunder other than the license as provided for above in Section 2. No right, license or interest to any trademark, trade name, service mark or other branding ("Marks") of WILEY or its licensors is granted bereunder, and you agree that you shall not assert any such right, license or interest with respect thereto.

- NEITHER WILEY NOR ITS LICENSORS MAKES ANY WARRANTY OR REPRESENTATION OF ANY KIND TO YOU OR ANY THIRD PARTY, EXPRESS, IMPLIED OR STATUTORY, WITH RESPECT TO THE MATERIALS OR THE ACCURACY OF ANY INFORMATION CONTAINED IN THE MATERIALS, DNCLUDING, WITHOUT LIMITATION, ANY IMPLIED WARRANTY OF MERCHANTABIITY, ACCURACY, SATISFACTORY QUALITY, FITNESS FOR A PARTICULAR PURPOSE, USABIIITY, INTEGRATION OR NON-INFRINGEMENT AND ALL SUCH WARRANTIES ARE HEREBY EXCLUDED BY WILEY AND ITS LICENSORS AND WAIVED BY YOU

- WILEY shall have the right to terminate this Agreement immediately upon breach of this Agreement by you.

- You shall indennify, defend and hold harmless WII.EY, its Licensors and their respective directors, officers, agents and employees, from and against any actual or threatened claims, demands, causes of action or proceedings arising from any breach of this Agreement by you.

- IN NO EVENT SHALL WILEY OR ITS LICENSORS BE LIABLE TO YOU OR ANY OTHER PARTY OR ANY OTHER PERSON OR ENTITY FOR ANY SPECIAL, CONSEQUENTIAL, INCIDENTAL, INDIRECT, EXEMPLARY OR PUNIIIVE DAMAGES, HOWEVER CAUSED, ARISING OUT OF OR IN CONNECTION WITH THE DOWNLOADING, PROVISIONING, VIEWING OR USE OF THE MATERIALS REGARDLESS OF THE FORM OF ACTION, WHETHER FOR BREACH OF CONTRACT, BREACH OF WARRANTY, TORT, NEGLIGENCE, INFRINGEMENT OR OTHERWISE (INCLUDING, WITHOUT LIMITATION, DAMAGES BASED ON LOSS OF PROFITS, DATA, FILES, USE, BUSINESS OPPORTUNITY OR CLAIMS OF THIRD PARTIES), AND WHETHER OR NOT THE PARTY HAS BEEN ADVISED OF THE POSSIBIITYY OF SUCH DAMAGES. THIS LIMITATION SHALL APPLY NOTWITHSTANDING ANY FAIIURE OF ESSENTIAL PURPOSE OF ANY LIMITED REMEDY PROVIDED HEREIN.

- Should any provision of this Agreement be held by a court of competent jurisdiction to be illegal, invalid, or unenforceable, that provision shall be deemed amended to achieve as nearly as possible the same economic effect as the original provision, and 
the legality, validity and enforceability of the remaining provisions of this Agreement shall not be affected or impaired thereby.

- The failure of either party to enforce any term or condition of this Agreement shall not constitute a waiver of either party's right to enforce each and every term and condition of this Agreement. No breach under this agreement shall be deemed waived or excused by either party unless such waiver or consent is in writing signed by the party granting such waiver or consent. The waiver by or consent of a party to a breach of any provision of this Agreement shall not operate or be construed as a waiver of or consent to any other or subsequent breach by such other party.

- This Agreement may not be assigned (including by operation of law or otherwise) by you without WILEY's prior written consent.

- Any fee required for this permission shall be non-refundable after thirty (30) days from receipt by the CCC.

- These terms and conditions together with CCC 2 s Billing and Payment terms and conditions (which are incorporated berein) form the entire agreement between you and WIILY concerning this licensing transaction and (in the absence of fraud) supersedes all prior agreements and representations of the parties, oral or written. This Agreement may not be amended except in writing signed by both parties. This Agreement shall be binding upon and inure to the benefit of the parties' successors, legal representatives, and authorized assigns.

- In the event of any conflict between your obligations established by these terms and conditions and those established by CCC 2 s Billing and Payment terms and conditions, these terms and conditions shall prevail.

- WILEY expressly reserves all rights not specifically granted in the combination of (i) the license details provided by you and accepted in the course of this licensing transaction, (ii) these terms and conditions and (iii) CCC $\theta_{s}$ Billing and Payment terms and conditions.

- This Agreement will be void if the Type of Use, Format, Circulation, or Requestor Type was misrepresented during the licensing process.

- This Agreement shall be governed by and construed in accordance with the laws of the State of New York, USA, without regards to such state ${ }^{\text {s }}$ conflict of law rules. Any legal action, suit or proceeding arising out of or relating to these Terms and Conditions or the breach thereof shall be instituted in a court of competent jurisdiction in New York County in the State of New York in the United States of America and each party hereby consents and submits to the personal jurisdiction of such court, waives any objection to verue in such court and consents to service of process by registered or certified mail, return receipt requested, at the last known address of such party. 


\section{WILEY OPEN ACCESS TERMS AND CONDITIONS}

Wiley Publisbes Open Access Articles in fully Open Access Journals and in Subscription journals offering Online Open. Although most of the fully Open Access joumals publish open access articles under the terms of the Creative Commons Attribution (CC BY) License only, the subscription journals and a few of the Open Access Joumals offer a choice of Creative Commons Licenses:: Creative Commons Attribution (CC-BY) license Creative Commons Attribution. Non-Commercial (CC-BY-NC) license and Creative Commons Attribution Non-Commercial-NoDerivs (CC-BYCNC-ND) License. The license type is clearly identified on the article.

Copyright in any research article in a journal published as Open Access under a Creative Commons License is retained by the author(s). Authors grant Wiley a license to publish the article and identify itself as the original publisber. Authors also grant any third party the right to use the article freely as long as its integrity is maintained and its original authors, citation details and publisher are identified as follows: [Title of Article/Author/Journal Title and Volume/Issue. Copyright (c) [year] [copyright owner as specified in the Jourual]. Links to the final article on Wiley $\partial_{5}$ website are encouraged where applicable.

\section{The Creative Commons Attribution License}

The Creative Commons Attribution License (CC-BY) allows users to copy, distribute and trarsmit an article, adapt the article and make commercial use of the article. The CC-BY license permits commercial and non-commercial re-use of an open access article, as long as the author is properly attributed.

The Creative Commons Attribution License does not affect the moral rights of authors, including without limitation the right not to have their work subjected to derogatory treatment. It also does not affect any other rights beld by authors or third parties in the article, including without limitation the rights of privacy and publicity. Use of the article must not assert or imply, whether implicitly or explicitly, any connection with, endorsement or sponsorship of such use by the author, publisher or any other party associated with the article.

For any reuse or distribution, users must include the copyright notice and make clear to others that the article is made available under a Creative Conmons Attribution license, linking to the relevant Creative Commons web page.

To the fullest extent permitted by applicable law, the article is made available as is and without representation or warranties of any kind whether express, implied, statutory or otherwise and including, without limitation, warranties of title, merchantability, fitness for a particular purpose, non-infringement, absence of defects, accuracy, or the presence or absence of enrors.

\section{Creative Commons Attribution Non-Commercial License}

The Creative Commons Attribution Non-Commercial (CC-BY-NC) License permits use, distribution and reproduction in any medium, provided the original work is properly cited 
and is not used for commercial purposes.(see below)

Creative Commons Attribution-Non-Commercial-NoDerivs License

The Creative Commons Attribution Non-Commercial-NoDerivs License (CC-BY-NC-ND) permits use, distribution and reproduction in any medium, provided the original work is properly cited, is not used for commercial purposes and no modifications or adaptations are made. (see below)

\section{Use by non-commercial users}

For non-commercial and non-promotional purposes, individual users may access, download, copy, display and redistribute to colleagues Wiley Open Access articles, as well as adapt, trarslate, text- and data-mine the content subject to the following conditions:

- The authors' moral rights are not compromised. These rights include the right of "paternity" (also krown as "attribution" - the right for the author to be identified as such) and "integrity" (the right for the author not to have the work altered in such a way that the author's reputation or integrity may be inmpugned).

- Where content in the article is identified as belonging to a third party, it is the obligation of the user to ensure that any reuse complies with the copyright policies of the owner of that content.

- If article content is copied, downloaded or otherwise reused for non-commercial research and education pupposes, a link to the appropriate bibliographic citation (authors, journal, article title, volume, issue, page numbers, DOI and the link to the definitive published version on Wiley Online Library) should be maintained. Copyright notices and disclaimers must not be deleted.

- Any translations, for which a prior translation agreement with Wiley has not been agreed, must prominently display the statement: "This is an unofficial translation of an article that appeared in a Wiley publication. The publisber has not endorsed this translation."

\section{Use by commercial "for-profit" organisations}

Use of Wiley Open Access articles for conmercial, promotional, or marketing purposes requires further explicit permission from Wiley and will be subject to a fee. Commercial purposes include:

- Copying or downloading of articles, or linking to such articles for further redistribution, sale or licensing;

- Copying, downloading or posting by a site or service that incorporates advertising with such content;

- The inclusion or incorporation of article content in other works or services (other than 
normal quotations with an appropriate citation) that is then available for sale or licensing, for a fee (for example, a compilation produced for marketing purposes, inclusion in a sales pack)

- Use of article content (other than normal quotations with appropriate citation) by forprofit organisations for promotional purposes

- Linking to article content in e-mails redistributed for promotional, marketing or educational purposes;

- Use for the purposes of monetary reward by means of sale, resale, licence, loan, transfer or other form of commercial exploitation such as marketing products

- Print reprints of Wiley Open Access articles can be purchased from: corroratesales

Further details can be found on Wiley Online Library

htto//olabout. wileys com/WileyCDA/Sectionlid-410895/html

Other Terms and Conditions:

v1.9

If you would like to pay for this license now, please remit this license along with your payment made payable to "COPYRIGHT CLEARANCE CENTER" otherwise you will be

invoiced within $\mathbf{4 8}$ hours of the license date. Payment should be in the form of a check or money order referencing your account number and this invoice number 501323078 . Once you receive your invoice for this order, you may pay your invoice by credit card. Please follow instructions provided at that time.

Make Payment To:

Copyright Clearance Center

Dept 001

P.0. Box 843006

Boston, MA 02284-3006

For suggestions or comments regarding this order, contact RightsLink Customer Supporti customercare ecopyright.com or +1-877-622-5543 (toll free in the US) or +1978-646-2777.

Gratis licenses (referencing $\mathbf{\$ 0}$ in the Total fleld) are free. Please retain this printable license for your reference. No payment is required. 


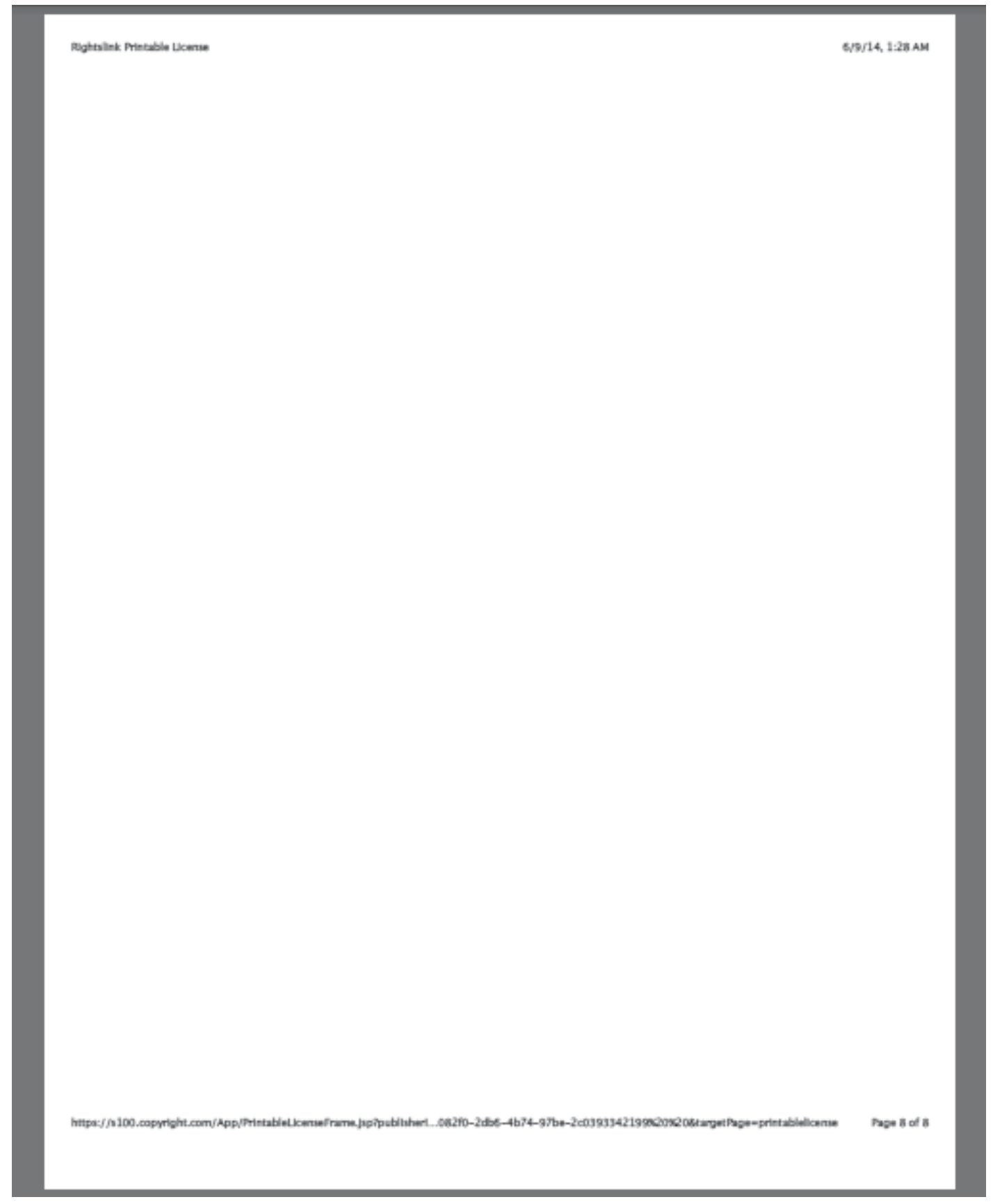




\section{B.7 Figure 3.7c Permission}

Wothilnk Mintuble Leanse

S1914, 1:31 AM

SPRINGER LICENSE

TERMS AND CONDITIONS

Jun 09, 2014

This is a License Agreement between Tayloria Adams ("You") and Springer ("Springer") provided by Copyright Clearance Center (" $\mathrm{CCC}$ "). The license consists of your order details, the terms and conditions provided by Springer, and the payment terms and conditions.

All payments must be made in full to CCC. For payment instructions, please see information listed at the bottom of this form.

Ucense Number

3404600912462

Ucense date

Jun 09, 2014

Licensed content publisher

Springer

Licensed content publication

Licensed content title

Licensed content author

Particle focusing in a contactless dielectrophoretic microfluidic chip with insulating structures

Licensed content date Chun-Ping Jen

Volume number

Jan 1, 2012

Issue number

18

Type of Use

11

Portion

Thesis/Dissertation

Author of this Springer

Figures

article

Order reference number

Original figure numbers

Title of your thesis /

dissertation

No

None

Figure $7 b$

Utilizing Dielectrophoresis to Determine the Physiological Differences of Eukaryotic Cells

Expected completion date Aug 2014

Estimated size(pages) $\quad 200$

Total

0.00 USD

Terms and Conditions

Introduction

The publisher for this copyrighted material is Springer Science + Business Media. By clicking "accept" in connection with completing this licensing transaction, you agree that the 
following terms and conditions apply to this transaction (along with the Billing and Payment terms and conditions established by Copyright Clearance Center, Inc. ("CCC"), at the time that you opened your Rightslink account and that are available at any time at htin///mwaccount copsrieht. com).

\section{Limited License}

With reference to your request to reprint in your thesis material on which Springer Science and Business Media control the copyright, permission is granted, free of charge, for the use indicated in your enquiry.

Licenses are for one-time use only with a maxinnum distribution equal to the rumber that you identified in the licensing process.

This License includes use in an electronic form, provided its password protected or on the uriversity's intranet or repository, including UMI (according to the definition at the Sherpa website: http: //www sherpa ac.uk/romeo/). For any other electronic use, please contact Springer at (permissions.dordrecht $₫$ springer.com or permissions heidelberg $@$ springer.com).

The material can only be used for the purpose of defending your thesis limited to uriversityuse only. If the thesis is going to be publisbed, permission needs to be re-obtained (selecting "book/textbook" as the type of use).

Although Springer holds copyright to the material and is entitled to negotiate on rights, this license is only valid, subject to a courtesy information to the author (address is given with the article/chapter) and provided it concerns original material which does not cany references to other sources (if material in question appears with credit to another source, authorization from that source is required as well).

Permission free of charge on this occasion does not prejudice any rights we might have to charge for reproduction of our copyrighted material in the future.

Altering/Modifying Material: Not Permitted

You may not alter or modify the material in any manner. Abbreviations, additions, deletions and/or any other alterations shall be made only with prior written authorization of the author(s) and/or Springer Science + Business Media. (Please contact Springer at (permissions dordrecht@springer.com or permissions heidelberg $@$ springer.com)

Reservation of Rights

Springer Science + Business Media reserves all rights not specifically granted in the combination of (i) the license details provided by you and accepted in the course of this licensing transaction, (ii) these terms and conditions and (iii) $\mathrm{CCO}$ s Billing and Payment terms and conditions.

Copyright Notice:Disclaimer

You must include the following copyright and permission notice in connection with any reproduction of the licensed material: "Springer and the original publisher /journal title, volume, year of publication, page, chapter/article title, name(s) of author(s), figure number(s), original copyright notice) is given to the publication in which the material was 
originally published, by adding; with kind permission from Springer Science and Business Media"

\section{Warranties: None}

Bxample 1: Springer Science + Business Media makes no representations or warranties with respect to the licensed material.

Bxample 2: Springer Science + Business Media makes no representations or warranties with respect to the licensed material and adopts on its own behalf the limitations and disclaimers established by CCC on its behalf in its Billing and Payment terms and conditions for this licensing transaction.

\section{Indemuity}

You hereby indemnify and agree to hold harmless Springer Science + Business Media and $\triangle C C$, and their respective officers, directors, employees and agents, from and against any and all claims arising out of your use of the licensed material other than as specifically authorized pursuant to this license.

No Transfer of License

This license is personal to you and may not be sublicensed, assigned, or transferred by you to any other person without Springer Science + Business Media's written permission.

No Amendment Bxcept in Writing

This license may not be amended except in a writing signed by both parties (or, in the case of Springer Science + Business Media, by CCC on Springer Science + Business Media's behalf).

Objection to Contrary Terms

Springer Science + Business Media hereby objects to any terms contained in any purchase order, acknowledgment, check endorsement or other writing prepared by you, which terms are inconsistent with these terms and conditions or CCC's Billing and Payment terms and conditions. These terms and conditions, together with $C C C$ 's Billing and Payment terms and conditions (which are incorporated berein), comprise the entire agreement between you and Springer Science + Business Media (and CCC) concening this licensing transaction. In the event of any conflict between your obligations established by these terms and conditions and those establisbed by $\mathrm{CCC}$ 's Billing and Payment terms and conditions, these terms and conditions shall control.

Jurisdiction

All disputes that may arise in connection with this present License, or the breach thereof, shall be settled exclusively by arbitration, to be beld in The Netherlands, in accordance with Dutch law, and to be conducted under the Rules of the 'Netherlands Arbitrage Instinut' (Netherlands Institute of Arbitration) $O R$ :

All disputes that may arise in connection with this present License, or the breach thereof, shall be setfled exclusively by arbitration, to be held in the Federal Republic of Germany, in accordance with German law. 
Other terms and conditions:

v1.3

If you would like to pay for this license now, please remit this license along with your payment made payable to "COPYRIGHT CLEARANCE CENTER" otherwise you will be invoiced within 48 hours of the license date. Payment should be in the form of a check or money order referencing your account number and this invoice number 501323081. Once you recelve your invoice for this order, you may pay your invoice by credit card. Please follow instructions provided at that time.

Make Payment Tot

Copyright Clearance Center

Dept 001

P.O. Box 843006

Boston, MA 02284-3006

For suggestions or comments regarding this order, contact RightsLink Customer Supporti customencareecopyright.com or +1-877-622-5543 (toll free in the US) or +1 978-646-2777.

Gratis licenses (referencing $\$ 0$ in the Total field) are free. Please retain this printable license for your reference. No payment is required. 


\section{B.8 Figure 3.7d Permission}

Moghtink Mintuble Ucense

JOHN WILEY AND SONS LICENSE

TERMS AND CONDITIONS

Jun 09,2014

This is a License Agreement between Tayloria Adams ("You") and John Wiley and Sons ("John Wiley and Sons") provided by Copyright Clearance Center ("CCC"). The license consists of your order details, the terms and conditions provided by John Wiley and Sons, and the payment terms and conditions.

All payments must be made in full to CCC. For payment instructions, please see information listed at the bottom of this form.

License Number

Uicense date

Uicensed content publisher

Uicensed content publication

Uicensed content title

Licensed copyright line

Licensed content author

Licensed content date

Start page

End page

Type of use

Requestor type

Format

Portion

Number of figures/tables

Original Wiley figure/table number(s)

Will you be translating?

Title of your thesis /

dissertation

Expected completion date

Expected size (number of pages)
3404601099129

Jun 09, 2014

John Wiley and Sons

Electrophoresis

Dynamic microparticle manipulation with an electrocsmotic flow gradient in low-frequency altemating current dielectrophoresis

9 2013 WILFY-VCH Verlag GmbH \& Co. KGaA, Weinheim

Aytug Gencoglu,David Olney,Alexandra LaLonde,Karuna S. Koppula,Blanca H. Lapizco-Encinas

Dec 5, 2013

362

373

Dissertation/Thesis

University/Academic

Print and electronic

Figure/table

1

Figure 5e-E

No

Utilizing Dielectrophoresis to Determine the Physiological Differences of Eukaryotic Cells

Aug 2014

200 
Terms and Conditions

\section{TERMS AND CONDITIONS}

This copyriehted material is owned by or exclusively licensed to Jobn Wiley \& Sons, Inc. or one of its group comparies (each a"Wiley Company") or handled on behalf of a society with which a Wiley Company has exclusive publishing rights in relation to a particular work (collectively "WILEY"). By clicking 2 accept in conrection with completing this licensing transaction, you agree that the following terms and conditions apply to this transaction (along with the billing and payment terms and conditions established by the Copyright Clearance Center Inc., ("CCC's Billing and Payment terms and conditions"), at the time that you opened your Rightslink account (these are available at any time at hitin//mavaccount copsrieht. com).

\section{Terms and Conditions}

- The materials you have requested permission to reproduce or reuse (the "Wiley Materials") are protected by copyright.

- You are hereby granted a personal, non-exclusive, non-sub licensable (on a standalone basis), non-transferable, worldwide, limited license to reproduce the Wiley Materials for the purpose specified in the licensing process. This license is for a onetime use only and limited to any maximum distribution number specified in the license. The first instance of republication or reuse granted by this licence must be completed within two years of the date of the grant of this licence (although copies prepared before the end date may be distributed thereafter). The Wiley Materials shall not be used in any other manner or for any other purpose, beyond what is granted in the license. Permission is granted subject to an appropriate acknowledgement given to the author, title of the material/book/journal and the publisher. You shall also duplicate the copyright notice that appears in the Wiley publication in your use of the Wiley Material. Permission is also granted on the understanding that nowhere in the text is a previously published source acknowledged for all or part of this Wiley Material. Any third party content is expressly excluded from this permission.

- With respect to the Wiley Materials, all rights are reserved. Except as expressly granted by the terms of the license, no part of the Wiley Materials may be copied, modified, adapted (except for minor reformatting required by the new Publication), translated, reproduced, transferred or distributed, in any form or by any means, and no derivative works may be made based on the Wiley Materials without the prior permission of the respective copyright owner. You may not alter, remove or suppress in any manner any copyright, trademark or other notices displayed by the Wiley Materials. You may not license, rent, sell, loan, lease, pledge, offer as security, transfer or assign the Wiley Materials on a stand-alone basis, or any of the rights granted to you bereunder to any other person. 
- The Wiley Materials and all of the intellectual property rights therein shall at all times remain the exclusive property of Jobn Wiley \& Sons Inc, the Wiley Comparies, or their respective licensors, and your interest therein is only that of having possession of and the right to reproduce the Wiley Materials pursuant to Section 2 herein during the continuance of this Agreement. You agree that you own no right, title or interest in or to the Wiley Materials or any of the intellectual property rights therein. You shall have no rights hereunder other than the license as provided for above in Section 2. No right, license or interest to any trademark, trade name, service mark or other branding ("Marks") of WILEY or its licensors is granted bereunder, and you agree that you shall not assert any such right, license or interest with respect thereto.

- NEITHER WILEY NOR ITS LICENSORS MAKES ANY WARRANTY OR REPRESENTATION OF ANY KIND TO YOU OR ANY THIRD PARTY, EXPRESS, IMPLIED OR STATUTORY, WITH RESPECT TO THE MATERIALS OR THE ACCURACY OF ANY INFORMATION CONTAINED IN THE MATERIALS, INCLUDING, WITHOUT LIMITATION, ANY IMPLIED WARRANTY OF MERCHANTABILITY, ACCURACY, SATISFACTORY QUALITY, FITNESS FOR A PARTICULAR PURPOSE, USABIITY, INTEGRATION OR NON-INFRINGEMENT AND ALL SUCH WARRANTIES ARE HEREBY BXCLUDED BY WILEY AND ITS LICENSORS AND WAIVED BY YOU

- WILEY shall have the right to terminate this Agreement immediately upon breach of this Agreement by you.

- You shall indennify, defend and hold harmless WILEY, its Licensors and their respective directors, officers, agents and employees, from and against any actual or threatened claims, demands, causes of action or proceedings arising from any breach of this Agreement by you.

- IN NO EVENT SHALL WILEY OR ITS LICENSORS BE LIABLE TO YOU OR ANY OTHER PARTY OR ANY OTHER PERSON OR ENTITY FOR ANY SPECIAL, CONSEQUENTIAL, INCIDENTAL, INDIRECT, EXEMPLARY OR PUNIIIVE DAMAGES, HOWBVER CAUSED, ARISING OUT OF OR IN CONNECTION WITH THE DOWNLOADING, PROVISIONING, VIEWING OR USE OF THE MATERIALS REGARDLESS OF THE FORM OF ACTION, WHETHER FOR BREACH OF CONTRACT, BREACH OF WARRANTY, TORT, NEGLIGENCE, INFRINGEMENT OR OTHERWISE (INCLUDING, WITHOUT LIMITATION, DAMAGES BASED ON LOSS OF PROFITS, DATA, FILES, USE, BUSINESS OPPORTUNITY OR CLAIMS OF THIRD PARTIES), AND WHETHER OR NOT THE PARTY HAS BEEN ADVISED OF THE POSSIBIIITY OF SUCH DAMAGES. THIS LIMITATION SHALL APPLY NOTWITHSTANDING ANY FAILURE OF ESSENTIAL PURPOSE OF ANY LIMITED REMEDY PROVIDED HERENN.

- Should any provision of this Agreement be held by a court of competent jurisdiction to be illegal, invalid, or unenforceable, that provision shall be deemed amended to 
achieve as nearly as possible the same economic effect as the original provision, and the legality, validity and enforceability of the remaining provisions of this Agreement shall not be affected or impaired thereby.

- The failure of either party to enforce any term or condition of this Agreement shall not constitute a waiver of either party's right to enforce each and every term and condition of this Agreement. No breach under this agreement shall be deemed waived or excused by either party unless such waiver or consent is in writing signed by the party granting such waiver or consent. The waiver by or consent of a party to a breach of any provision of this Agreement shall not operate or be construed as a waiver of or consent to any other or subsequent breach by such other party.

- This Agreement may not be assigned (including by operation of law or otherwise) by you without WILEY's prior written consent.

- Any fee required for this permission shall be non-refundable after thirty (30) days from receipt by the CCC.

- These terms and conditions together with CCC 8 s Biling and Payment terms and conditions (which are incorporated berein) form the entire agreement between you and WIIEY concening this licensing transaction and (in the absence of fraud) supersedes all prior agreements and representations of the parties, oral or written. This Agreement may not be amended except in writing signed by both parties. This Agreement shall be binding upon and inure to the benefit of the parties' successors, legal representatives, and authorized assigns.

- In the event of any conflict between your obligations established by these terms and conditions and those established by CCC 0 s Billing and Payment terms and conditions, these terms and conditions shall prevail.

- WILEY expressly reserves all rights not specifically granted in the combination of (i) the license details provided by you and accepted in the course of this licensing transaction, (ii) these terms and conditions and (iii) $\mathrm{CCC} \mathrm{v}_{\mathrm{s}}$ Billing and Payment terms and conditions.

- This Agreement will be void if the Type of Use, Format, Circulation, or Requestor Type was misrepresented during the licensing process.

- This Agreement shall be governed by and construed in accordance with the laws of the State of New York, USA, without regards to such state 0 s conflict of law rules. Any legal action, suit or proceeding arising out of or relating to these Terms and Conditions or the breach thereof shall be instituted in a court of competent jurisdiction in New York County in the State of New York in the United States of America and each party hereby consents and submits to the personal jurisdiction of such court, waives any objection to verue in such court and consents to service of process by registered or certified mail, retum receipt requested, at the last known address of such party. 


\section{WIIEY OPEN ACCESS TERMS AND CONDITIONS}

Wiley Publisbes Open Access Articles in fully Open Access Journals and in Subscription journals offering Online Open. Although most of the fully Open Access joumals publish open access articles under the terms of the Creative Commons Attribution (CC BY) License only, the subscription journals and a few of the Open Access Journals offer a choice of Creative Commons Licenses:: Creative Commons Attribution (CC-BY) license Creative Commons Antribution.Non-Commercial,(CC-BY-NC) license and Creative-Commons. Attribution Non-Commercial-NoDerivs (CC-BYCNC-ND) License. The license type is clearly identified on the article.

Copyright in any research article in a journal published as Open Access under a Creative Commons License is retained by the author(s). Authors grant Wiley a license to publish the article and identify itself as the original publisber. Authors also grant any third party the right to use the article freely as long as its integrity is maintained and its original authors, citation details and publisher are identified as follows: [Title of Article/Author/Journal Title and Volume/Issue. Copyright (c) [year] [copyright owner as specified in the Jourual]. Links to the final article on Wiley 2 s website are encouraged where applicable.

\section{The Creative Commons Attribution License}

The Creative Commons Attribution License (CC-BY) allows users to copy, distribute and transmit an article, adapt the article and make commercial use of the article. The CC-BY license permits commercial and non-commercial re-use of an open access article, as long as the author is properly attributed.

The Creative Commons Attribution License does not affect the moral rights of authors, including without limitation the right not to have their work subjected to derogatory treatment. It also does not affect any other rights beld by authors or third parties in the article, including without limitation the rights of privacy and publicity. Use of the article must not assert or imply, whether implicitly or explicitly, any connection with, endorsement or sponsorship of such use by the author, publisher or any other party associated with the article.

For any reuse or distribution, users must include the copyright notice and make clear to others that the article is made available under a Creative Commons Attribution license, linking to the relevant Creative Commons web page.

To the fullest extent permitted by applicable law, the article is made avaliable as is and without representation or wanranties of any kind whether express, implied, statutory or otherwise and including, without limitation, wanranties of title, merchantability, fitness for a particular purpose, non-infringement, absence of defects, accuracy, or the presence or absence of enrors.

\section{Creative Commons Attribution Non-Commercial License}


The Creative Commons Attribution Non-Commercial (CC-BY-NC) License permits use, distribution and reproduction in any medium, provided the original work is properly cited and is not used for commercial purposes.(see below)

\section{Creative Commons Attribution-Non-Commercial-NoDerivs License}

The Creative Commons Attribution Non-Commercial-NoDeriws License (CC-BY-NC-ND) permits use, distribution and reproduction in any medium, provided the original work is properly cited, is not used for commercial purposes and no modifications or adaptations are made. (see below)

\section{Use by non-commercial users}

For non-commercial and non-promotional purposes, individual users may access, download, copy, display and redistribute to colleagues Wiley Open Access articles, as well as adapt, translate, text- and data-mine the content subject to the following conditions:

- The authors' moral rights are not compromised. These rights include the right of "paternity" (also krown as "attribution" - the right for the author to be identified as such) and "integrity" (the right for the author not to have the work altered in such a way that the author's reputation or integrity may be impugned).

- Where content in the article is identified as belonging to a third party, it is the obligation of the user to ensure that any reuse complies with the copyright policies of the owner of that content.

- If article content is copied, downloaded or otherwise reused for non-commercial research and education purposes, a link to the appropriate bibliographic citation (authors, journal, article title, volume, issue, page numbers, DOI and the link to the definitive published version on Wiley Online Library) should be maintained. Copyright notices and disclaimers must not be deleted.

- Any translations, for which a prior translation agreement with Wiley has not been agreed, must prominently display the statement: "This is an unofficial trarslation of an article that appeared in a Wiley publication. The publisber has not endorsed this translation."

\section{Use by commercial "for-profit" organisations}

Use of Wiley Open Access articles for conmercial, promotional, or marketing purposes requires further explicit permission from Wiley and will be subject to a fee. Commercial purposes include:

- Copying or downloading of articles, or linking to such articles for further redistribution, sale or licensing;

- Copying, downloading or posting by a site or service that incorporates advertising with such content; 
- The inclusion or incorporation of article content in other works or services (other than normal quotations with an appropriate citation) that is then available for sale or licensing, for a fee (for example, a compilation produced for marketing purposes, inclusion in a sales pack)

- Use of article content (other than normal quotations with appropriate citation) by forprofit organisations for promotional purposes

- Linking to article content in e-mails redistributed for promotional, marketing or educational purposes;

- Use for the purposes of monetary reward by means of sale, resale, licence, loan, transfer or other form of commercial exploitation such as marketing products

- Print reprints of Wiley Open Access articles can be purchased from: corporatesales (1) wiley.com

Further details can be found on Wiley Online Library

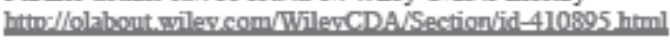

Other Terms and Conditions:

v1.9

If you would like to pay for this license now, please remit this license along with your payment made payable to "COPYRIGHT CLEARANCE CENTER" otherwise you will be invoiced within $\mathbf{4 8}$ hours of the license date. Payment should be in the form of a check or money order referencing your account number and this invoice number 501323082. Once you recelve your involce for this order, you may pay your invoice by credit card. Please follow instructions provided at that time.

Make Payment To:

Copyright Clearance Center

Dept 001

P.O. Box 843006

Boston, MA 02284-3006

For suggestions or comments regarding this order, contact RightsLink Customer Supporti customercarefecomoriehthcom or +1-877-622-55.43 (toll free in the US) or +1 978-646-2777.

Gratis licenses (referencing $\$ 0$ in the Total fleld) are free. Please retain this printable license for your reference. No payment is required. 


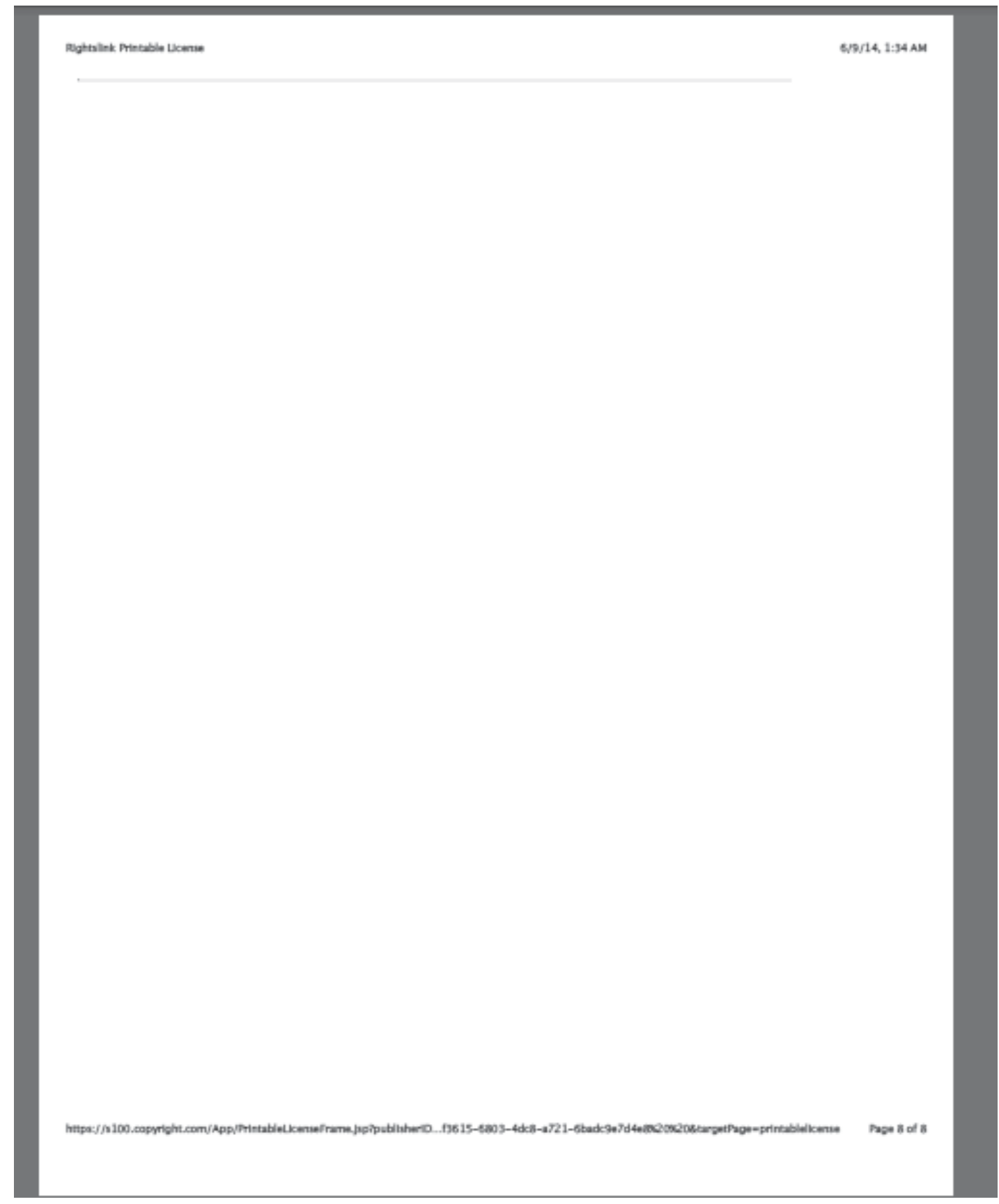




\section{B.9 Figure 3.8 Permission}

$$
\text { ELSEVIER LICENSE }
$$

TERMS AND CONDITIONS

This is a License Agreement between Tayloria Adams ("You") and Elsevier ("Elsevier") provided by Copyright Clearance Center ("CCC"). The license consists of your order details, the terms and conditions provided by Elsevier, and the payment terms and conditions.

All payments must be made in full to CCC. For payment instructions, please see information listed at the bottom of this form.

Supplier

Elsevier Limited

The Boulevard, Langford Lane

Kidlington,Oxdord, OxS 1GB, UK

Registered Company

1982084

Number

Tayloria Adams

Customer name

46573 Main St, Apt 3

Customer address

Dodgeville, MI 49921

License number

3405520294352

License date

Jun 10, 2014

Licensed content publisher

Elsevier

Licensed content publication

Licensed content title

Sensors and Actuators B: Chemical

Licensed content author dielectrophoresis

Licensed content author

Sander van den Driesche, Vlvek Ras, Dietmar Puchberger-

Licensed content date Enengl, Wojclech Witarski,Michael J. Veliekoop

Licensed content volume

31 July 2012

number

170

Uicensed content issue

None

number

Number of pages 8

Start Page $\quad 207$

End Page 214

Type of Use reuse in a thess/dissertation

Portion

Number of flgures/tables

/ilustrations

Format

figures/tables/illustrations

2

both print and electronic

Are you the author of this

Ekevier article?

Will you be translating? 
Estimated stae (number of

pages)

Elsevier VAT number

Aug 2014

200

Permiselons price

GB 494627212

VAT/Local Sales Tax 0.00 USD

Total

Terms and Conditions

\section{INTRODUCTION}

1. The publisher for this copyrighted material is Elsevier. By clicking "accept" in connection with completing this licensing transaction, you agree that the following terms and conditions apply to this transaction (along with the Billing and Payment terms and conditions established by Copyright Clearance Center, Inc. ("CCC"), at the time that you opened your Rightslink account and that are available at any time at http://myaccount.copyright.com).

\section{GENERAL TERMS}

2. Elsevier hereby grants you permission to reproduce the aforementioned material subject to the terms and conditions indicated.

3. Acknowledgement. If any part of the material to be used (for example, figures) has appeared in our publication with credit or acknowledgement to another source, permission must also be sought from that source. If such pennission is not obtained then that material may not be included in your publication/copies. Suitable acknowledgement to the source must be made, either as a footnote or in a reference list at the end of your publication, as folows:

"Reprinted from Publication title, Vol /edition number, Author(s). Title of article / title of chapter, Pages No., Copyright (Year), with permission from Elsevier [OR APPLICABLE SOCIETY COPYRIGHT OWNER]." Also Lancet special credit - "Reprinted from The Lancet, Vol number, Author(s). Title of article, Pages No., Copyright (Year), with permission from Elsevier."

4. Reproduction of this material is confined to the purpose and/or media for which permission is hereby given.

5. Altering/Modifying Material: Not Permitted. However figures and illustrations may be altered/adapted minimally to serve your work. Any other abbreviations, additions, deletions and/or any other alterations shall be made only with prior written authorization of Elsevier Ltd. (Please contact Elsevier at permissions@elsevier.com)

6. If the permission fee for the requested use of our material is waived in this instance, please be advised that your future requests for Elsevier materials may attract a fee. 
7. Reservation of Rights: Publisher reserves all rights not specifically granted in the combination of (i) the license details provided by you and accepted in the course of this licensing transaction, (ii) these terms and conditions and (iii) CCC's Billing and Paywent terms and conditions.

8. License Contingent Upon Payment: While you may exercise the rights licensed immediately upon issuance of the license at the end of the licensing process for the transaction, provided that you have disclosed complete and accurate details of your proposed use, no license is finally effective unless and until full payment is received from you (either by publisher or by $(\mathrm{CC})$ as provided in CCC's Billing and Payment terms and conditions. If full payment is not received on a timely basis, then any license preliminarily granted shall be deemed automatically revolked and shall be void as if never granted. Further, in the event that you breach any of these terms and conditions or any of $\mathrm{CCC}^{\prime}$ B Billing and Payment terms and conditions, the license is automatically revoked and shall be void as if never granted. Use of materials as described in a revolked license, as well as any use of the materials beyond the scope of an unrevoked license, may constitute copyright infringement and publisher reserves the right to take any and all action to protect its copyright in the materials.

9. Warranties: Publisher makes no representations or warranties with respect to the licensed material.

10. Indemnity: You hereby indemnify and agree to hold harmless publisher and $C C C$, and their respective officers, directors, employees and agents, from and against any and all claims arising out of your use of the licensed material other than as specifically authorized pursuant to this license.

11. No Transfer of License: This license is personal to you and may not be sublicensed, assigned, or transferred by you to any other person without publisher's written permission.

12. No Amendment Except in Writing: This license may not be amended except in a writing signed by both parties (or, in the case of publisher, by $\mathrm{CCC}$ on publisher's behalf).

13. Objection to Contrary Terms: Pablisher hereby objects to any terms contained in any purchase order, acknowledgment, check endorsement or other writing prepared by you, which terms are inconsistent with these terms and conditions or CCC's Billing and Payment terms and conditions. These terms and conditions, together with $\mathrm{CCC}^{\prime}$ s Billing and Payment terms and conditions (which are incorporated herein), comprise the entire agreement between you and publisher (and CCC) concerning this licensing transaction. In the event of any conflict between your obligations established by these terms and conditions and those established by CCC's Billing and Payment terms and conditions, these terms and conditions shall control.

14. Revocation: Elsevier or Copyright Clearance Center may deny the permissions described in this License at their sole discretion, for any reason or no reason, with a full refund payable to you. Notice of such denial will be made using the contact information provided by you. Failure to receive such notice will not alter or invalidate the denial. In no event will Elsevier or Copyright Clearance Center be responsible or liable for any costs, expenses or damage 
incurred by you as a result of a denial of your permission request, other than a refund of the amount(s) paid by you to Elsevier and/or Copyright Clearance Center for denied permissions.

\section{LIMITED LICENSE}

The following terms and conditions apply only to specific license types:

15. Translation: This permission is granted for non-exclusive world English rights only unless your license was granted for translation rights. If you licensed translation rights you may only translate this content into the languages you requested. A professional translator must perform all translations and reproduce the content word for word preserving the integrity of the article. If this license is to re-use 1 or 2 figures then permission is granted for non-exclusive world rights in all languages.

16. Posting licensed content on any Website: The following terms and conditions apply as folows: Licensing material from an Elsevier joumal: All content posted to the web site must maintain the copyright information line on the bottom of each image; A hyper-text must be included to the Homepage of the joumal from which you are licensing at http:/Www sciencedirect.com/science/joumal/sxxx or the Elsevier homepage for books at http://www.elsevier.com; Central Storage: This license does not include permission for a scanned version of the material to be stored in a central repository such as that provided by Heron/XanEdu.

Licensing material from an Elsevier book: A hyper-text link must be included to the Elsevier homepage at http://wrww.elsevier.com . All content posted to the web site must maintain the copyright information line on the bottom of each image.

Posting licensed content on Electronic reserve: In addition to the above the following clauses are applicable: The web site must be password-protected and made available only to bona fide students registered on a relevant course. This permission is granted for 1 year only. You may obtain a new license for furure website posting

For journal authors: the following clauses are applicable in addition to the above: Permission granted is limited to the author accepted manuscript version ${ }^{*}$ of your paper.

*Accepted Author Manuscript (AAM) Definition: An accepted author manuscript (AAM) is the author's version of the manuscript of an article that has been accepted for publication and which may include any author-incorporated changes suggested through the processes of submission processing, peer review, and editor-author communications. A.A.Ms do not include other publisher value-added contributions such as copy-editing, formatting, technical enhancements and (if relevant) pagination.

You are not allowed to download and post the published joumal article (whether PDF or HTML, proof or final version), nor may you scan the printed edition to create an electronic version. A hyper-text must be included to the Homepage of the journal from which you are

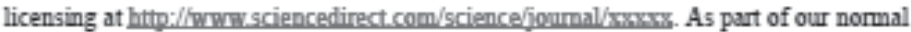
production process, you will receive an e-mail notice when your article appears on Elsevier's online service ScienceDirect (www sciencedirectcom). That e-mail will include 
the article's Digital Object Identifier (DOI). This number provides the electronic link to the published article and should be included in the posting of your personal version. We ask that you wait until you receive this e-mail and have the DOI to do any posting.

Posting to a repository: Authors may post their A.AM immediately to their employer's institutional repository for intermal use only and may make their manuscript publically available after the journal-specific embargo period has ended.

Please also refer to Elsevier's Article Posting Policy for further information.

18. For book authors the following clauses are applicable in addition to the above: Authors are permitted to place a brief summary of their work online only.. You are not allowed to download and post the published electronic version of your chapter, nor may you scan the printed edition to create an electronic version. Posting to a repository: Authors are permitted to post a summary of their chapter only in their institution's repository.

20. Thesis/Dissertation: If your license is for use in a thesis/dissertation your thesis may be submitted to your institution in either print or electronic form. Should your thesis be published commercially, please reapply for penmission. These requirements include permission for the Library and Archives of Canada to supply single copies, on demand, of the complete thesis and include permission for UMI to supply single copies, on demand, of the complete thesis. Should your thesis be published commercially, please reapply for permission.

\section{Elsevier Open Access Terms and Conditions}

Elsevier publishes Open Access articles in both its Open Access journals and via its Open Access articles option in subscription joumals.

Authors publishing in an Open Access joumal or who choose to make their article Open Access in an Elsevier subscription journal select one of the following Creative Commons user licenses, which define how a reader may reuse their work: Creative Commons Atrnibution License (CC BY). Creative Commons Attribution - Non Commercial -

ShareAlike (CC BY NC SA) and Creative Commons Attribution - Non Commercial - No Derivatives (CC BY NC ND)

Terms \& Conditions applicable to all Elsevier Open Access articles:

Any reuse of the article must not represent the author as endorsing the adaptation of the article nor should the article be modified in such a way as to damage the author's honour or reputation.

The author(s) must be appropriately credited.

If any part of the material to be used (for example, figures) has appeared in our publication with credit or acknowledgement to another source it is the responsibility of the user to ensure their reuse complies with the terms and conditions determined by the rights holder. 


\section{Additional Terms \& Conditious applicable to each Creative Commons user license:}

CC BY: You may distribute and copy the article, create extracts, abstracts, and other revised versions, adaptations or derivative works of or from an article (such as a translation), to include in a collective work (such as an anthology), to text or data mine the article, including for commercial purposes without permission from Elsevier

CC BY NC SA: For non-commercial purposes you may distribute and copy the article, create extracts, abstracts and other revised versions, adaptations or derivative works of or from an article (such as a translation), to include in a collective work (such as an anthology). to text and data mine the article and license new adaptations or creations under identical terms without permission from Elsevier

CC BY NC ND: For non-commercial purposes you may distribute and copy the article and include it in a collective work (such as an anthology), provided you do not alter or modify the article, without permission from Elsevier

Any commercial reuse of Open Access articles published with a CC BY NC SA or CC BY NC ND license requires permission from Elsevier and will be subject to a fee.

Commercial reuse includes:

Promotional purposes (advertising or marketing)

Commercial exploitation (e.g. a product for sale or loan)

Systematic distribution (for a fee or free of charge)

Please refer to Elsevier's Open Access Policy for further information.

\section{Other Conditions:}

v1.7

If you would like to pay for this license now, please remit this license along with you payment made payable to "COPYRIGHT CLEARANCE CENTER" otherwise you will be payment made payable to "COPYRIGHT CLEARANCE CENTER" otherwise you will be
involced within 48 hours of the license date. Payment should be in the form of a check Involced within $\mathbf{4 8}$ hours of the license date. Payment should be in the form of a check
or money order referending your account number and this involice number 501324860 . Once you receive your invoice for this order, you may pay your invoice by credit card. Please follow instructions provided at that time.

Make Payment To:

Copyright Clearance Center

Dept 001

P.O. Box 843006

Boston, MA 02284-3006 
For suggestions or comments regarding this order, contact RightsLink Customer Support! customercaree copyright.com or +1-877-622-5543 (toll free in the US) or $+1-978-646-2777$.

Gratis licenses (referending $\$ 0$ in the Total fleid) are free. Please retain this printable license for your reference. No payment is required. 


\section{B.10 Figure 3.9 Permission}

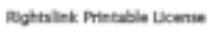

s/op14, $5.56 \mathrm{~m}$

ELSEVIER LICENSE

TERMS AND CONDITIONS

Aug 10, 2014

This is a License A reement between Tayloria Adams ("You") and Elsevier ("Elsevier") provided by Copyright Clearance Center (" $\mathrm{CCC}$ "). The license consists of your order details, the terms and conditions provided by Elsevier, and the payment terms and conditions.

All payments must be made in full to CCC. For payment instructions, please see information listed at the bottom of this form.

Supplier

Elsevier Limited

The Boulevard, Langford Lane

Kidlington, Oxford, $0 \times 5$ 1GB,UK

Registered Company $\quad 1982084$

Number

Customer name

Tayloria Adams

Customer address

46573 Main St, Apt 3

Dodgeville, MI 49921

Ucense number

3445561073137

License date

Aug 10, 2014

Licensed content publisher

Elsevier

Licensed content publication

Licensed content title

Plasma membrane characterization, by scanning electron

microscopy, of multipotent myoblasts-derived populations sorted using dielectrophoresis

Uicensed content author

Massimo Muratore, Steve Mitchell,Martin Waterfall

Licensed content date

6 September 2013

Ucensed content volume

438

number

Licensed content issue

number

Number of pages 7

Start Page 666

End Page $\quad 672$

Type of Use reuse in a thesis/dissertation

Portion figures/tables/illustrations

Number of 
figures/tables/illustrations

Format

Are you the author of this

Elsevier artide?

Will you be translating?

Title of your

thesis/dissertation

Expected completion date

Estimated size (number of

pages)

Elsevier VAT number

both print and electronic

Permissicns price

VAT/Local Sales Tax

Total

No

No

Utilizing Dielectrophoresis to Determine the Physiological Differences of Eukaryotic Cells

Terms and Conditions

Aug 2014

200

GB 494627212

0.00 USD

0.00 USD / $0.00 \mathrm{GBP}$

0.00 USD

\section{INTRODUCTION}

1. The publisber for this copyrighted material is Elsevier. By clicking "accept" in connection with completing this licensing transaction, you agree that the following terms and conditions apply to this transaction (along with the Billing and Payment terms and conditions established by Copyright Clearance Center, Inc. ("CCC"), at the time that you opened your Rightslink account and that are available at any time at httm//muraccosutit. copvrieht.eom).

\section{GENERAL TERMS}

2. Elsevier bereby grants you permission to reproduce the aforementioned material subject to the terms and conditions indicated.

3. Acknowledgement: If any part of the material to be used (for example, figures) has appeared in our publication with credit or acknowledgement to another source, permission must also be sought from that source. If such permission is not obtained then that material may not be included in your publication/copies. Suitable ackrowledgement to the source must be made, either as a footnote or in a reference list at the end of your publication, as follows:

"Reprinted from Publication title, Vol /edition number, Author(s), Title of article / title of chapter, Pages No., Copyright (Year), with permission from Elsevier [OR APPLICABLE SOCIETY COPYRIGHT OWNBR]." Also Lancet special credit - "Reprinted from The Lancet, Vol. number, Author(s), Title of article, Pages No., Copyright (Year), with permission from Elsevier."

4. Reproduction of this material is confined to the purpose and/or media for which permission is bereby given. 
5. Altering/Modifying Material: Not Permitted. However figures and illustrations may be altered/adapted minimally to serve your work. Any other abbreviations, additions, deletions and/or any other alterations shall be made only with prior written authorization of Blsevier Ltd. (Please contact Blsevier at permissions@elsevier.com)

6. If the permission fee for the requested use of our material is waived in this instance, please be advised that your future requests for Elsevier materials may attract a fee.

7. Reservation of Rights: Publisher reserves all rights not specifically granted in the combination of (i) the license details provided by you and accepted in the course of this licensing transaction, (ii) these terms and conditions and (iii) $0 \mathrm{CO}$ s Billing and Payment terms and conditions.

8. License Contingent Upon Payment: While you may exercise the rights licensed immediately upon issuance of the license at the end of the licensing process for the transaction, provided that you have disclosed complete and accurate details of your proposed use, no license is finally effective unless and until full payment is received from you (either by publisher or by $(C C)$ ) as provided in $\mathrm{CCC}^{\prime}$ Billing and Payment terms and conditions. If full payment is not received on a timely basis, then any license preliminarily granted shall be deemed automatically revoked and shall be void as if never granted. Further, in the event that you breach any of these terms and conditions or any of $\mathrm{CCO}_{\text {s }}$ Billing and Payment terms and conditions, the license is automatically revolked and shall be void as if never granted. Use of materials as described in a revolsed license, as well as any use of the materials beyond the scope of an uruevoked license, may constitute copyright infringement and publisber reserves the right to take any and all action to protect its copyright in the materials.

9. Warranties: Publisher makes no representations or warranties with respect to the licensed material.

10. Indennity: You hereby indemnify and agree to hold harmless publisher and CCC, and their respective officers, directors, employees and agents, from and against any and all claims arising out of your use of the licensed material other than as specifically autborized pursuant to this license.

11. No Transfer of License: This license is personal to you and may not be sublicensed, assigned, or transferred by you to any other person without publisher's written permission.

12. No Amendment Bxcept in Writing: This license may not be amended except in a writing signed by both parties (or, in the case of publisher, by CCC on publisher's behalf).

13. Objection to Contrary Terms: Publisher hereby objects to any terms contained in any purchase order, acknowledgment, check endorsement or other writing prepared by you, which terms are inconsistent with these terms and conditions or $\mathrm{CCO}$ s Billing and Payment terms and conditions. These terms and conditions, together with $\mathrm{CCC}^{\prime}$ B Billing and Payment terms and conditions (which are incorporated herein), comprise the entire agreement between you and publisber (and CCC) concening this licensing transaction. In the event of any conflict between your obligations established by these terms and conditions and those 
established by $\mathrm{CCO}_{5}$ Billing and Payment terms and conditions, these terms and conditions shall control.

14. Revocation: Elsevier or Copyright Clearance Center may deny the permissions described in this License at their sole discretion, for any reason or no reason, with a full refund payable to you. Notice of such denial will be made using the contact information provided by you. Failure to receive such notice will not alter or invalidate the denual. In no event will Elsevier or Copyright Clearance Center be responsible or liable for any costs, expenses or damage incurred by you as a result of a denial of your permission request, other than a refund of the amount(s) paid by you to Elsevier and/or Copyright Clearance Center for denied permissions.

\section{LIMITED LICENSE}

The following terms and conditions apply only to specific license types:

15. Translation: This permission is granted for non-exclusive world English rights only unless your license was granted for translation rights. If you licensed translation rights you may only translate this content into the languages you requested. A professional translator must perform all translations and reproduce the content word for word preserving the integrity of the article. If this license is to re-use 1 or 2 figures then permission is granted for non-exclusive world rights in all languages.

16. Posting licensed content on any Website: The following terms and conditions apply as follows: Licensing material from an Elsevier journal: All content posted to the web site must maintain the copyright information line on the bottom of each image; A hyper-text must be included to the Homepage of the journal from which you are licensing at

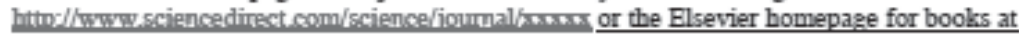
hitp $/ /$ www elseviercom; Central Storage: This license does not include permission for a scanned version of the material to be stored in a central repository such as that provided by HerorVXanBdu.

Licensing material from an Elsevier book: A hyper-text link must be included to the Elsevier homepage at $h(t n-/ / w w w e l s e v i e r c o m$. All content posted to the web site must maintain the copyright information line on the bottom of each image.

Posting licensed content on Electronic reserve: In addition to the above the following clauses are applicable: The web site must be password-protected and made available only to bona fide students registered on a relevant course. This permission is granted for 1 year only. You may obtain a new license for future website posting.

For journal authors: the following clauses are applicable in addition to the above: Permission granted is limited to the author accepted manuscript version ${ }^{*}$ of your paper.

*Accepted Author Manuscript (AAM) Definition: An accepted author manuscript (A.AM) is the author's version of the manuscript of an article that has been accepted for publication and which may include any author-incorporated changes suggested through the processes of submission processing, peer review, and editor-author communications. A.M.Ms do not 
include other publisher value-added contributions such as copy-editing, formatting, technical enhancements and (if relevant) pagination.

You are not allowed to download and post the published journal article (whether PDF or HTML, proof or final version), nor may you scan the printed edition to create an electronic version. A hyper-text must be included to the Homepage of the journal from which you are

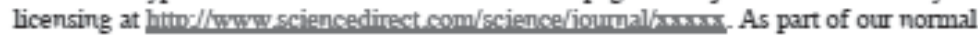
production process, you will receive an e-mail notice when your article appears on Elsevier's online service ScienceDirect (www sciencedirect.com). That e-mail will include the article's Digital Object Identifier (DOI). This number provides the electronic link to the published article and should be included in the posting of your personal version. We ask that you wait until you receive this e-mail and have the DOI to do any posting.

Posting to a repository: Authors may post their AAM immediately to their employer's institutional repository for internal use only and may make their manuscript publically available after the journal-specific embargo period has ended.

Please also refer to Elsevier's Article Postine Policy for further information.

18. For book authors the following clauses are applicable in addition to the above: Authors are permitted to place a brief summary of their work online only.. You are not allowed to download and post the published electronic version of your chapter, nor may you scan the printed edition to create an electronic version. Posting to a repository: Autbors are permitted to post a summary of their chapter only in their institution's repository.

20. Thesis/Dissertation: If your license is for use in a thesis/dissertation your thesis may be submitted to your institution in either print or electronic form. Should your thesis be published commercially, please reapply for permission. These requirements include permission for the Library and Archives of Canada to supply single copies, on demand, of the complete thesis and include permission for UMI to supply single copies, on demand, of the complete thesis. Should your thesis be published commercially, please reapply for permission.

\section{Elsevier Open Access Terms and Conditions}

Elsevier publishes Open Access articles in both its Open Access journals and via its Open Access articles option in subscription journals.

Authors publishing in an Open Access journal or who choose to make their article Open Access in an Elsevier subscription journal select one of the following Creative Commons user licenses, which define how a reader may reuse their work: Creative Commons Attribution License (CC BY), Creative Commons Attribution - Non Commercial ShareAlike (CC BY NC SA) and Creative Commons Attribution - Non Commercial - No Derivatives (CC BYNC ND)

Terms \& Conditions applicable to all Elsevier Open Access articles: 
Any reuse of the article must not represent the author as endorsing the adaptation of the article nor should the article be modified in such a way as to damage the author"s honour or reputation.

The author(s) must be appropriately credited.

If any part of the material to be used (for example, figures) has appeared in our publication with credit or acknowledgement to another source it is the responsibility of the user to ensure their reuse complies with the terms and conditions determined by the rights holder.

Additional Terms \& Conditions applicable to each Creative Commons user license:

CC BY: You may distribute and copy the article, create extracts, abstracts, and other revised versions, adaptations or derivative works of or from an article (such as a translation), to include in a collective work (such as an anthology), to text or data mine the article, including for conmercial purposes without permission from Elsevier

CC BY NC SA: For non-commercial purposes you may distribute and copy the article, create extracts, abstracts and other revised versions, adaptations or derivative works of or from an article (such as a translation), to include in a collective work (such as an anthology), to text and data mine the article and license new adaptations or creations urder identical terms without permission from Elsevier

CC BY NC ND: For non-commercial purposes you may distribute and copy the article and include it in a collective work (such as an anthology), provided you do not alter or modify the article, without permission from Blsevier

Any commercial reuse of Open Access articles published with a CC BY NC SA or CC BY NC ND license requires permission from Elsevier and will be subject to a fee.

Commercial reuse includes:

Promotional purposes (advertising or marketing)

Commercial exploitation (e.g. a product for sale or loan)

Systematic distribution (for a fee or free of charge)

Please refer to Blsevier's Open. Access. Policy for further information.

\section{Other Conditions:}

vl.6

You will be invoiced within $\mathbf{4 8}$ hours of this transaction date. You may pay your invoice 
by credit card upon receipt of the invoice for this transaction. Please follow instructions provided at that time.

To pay for this transaction now; please remit a copy of this document along with your payment. Payment should be in the form of a check or money order referencing your account number and this involce number RLNKS01373864.

Make payments to "COPYRIGHT CLEARANCE CENTER" and send to:

Copyright Clearance Center

\section{Dept 001}

P.O. Box 843006

Boston, MA 02284-3006

Please disregard electronic and mailed copies if you remit payment in advance.

Questions? customercaref comrilaht.com or +1-855-239-3415 (toll free in the US) or

+1-978-646-2777.

Gratis licenses (referencing $\$ 0$ in the Total fleld) are free. Please retain this printable license for your reference. No payment is required. 


\section{B.11 Figure 3.10 and 3.11 Permission}

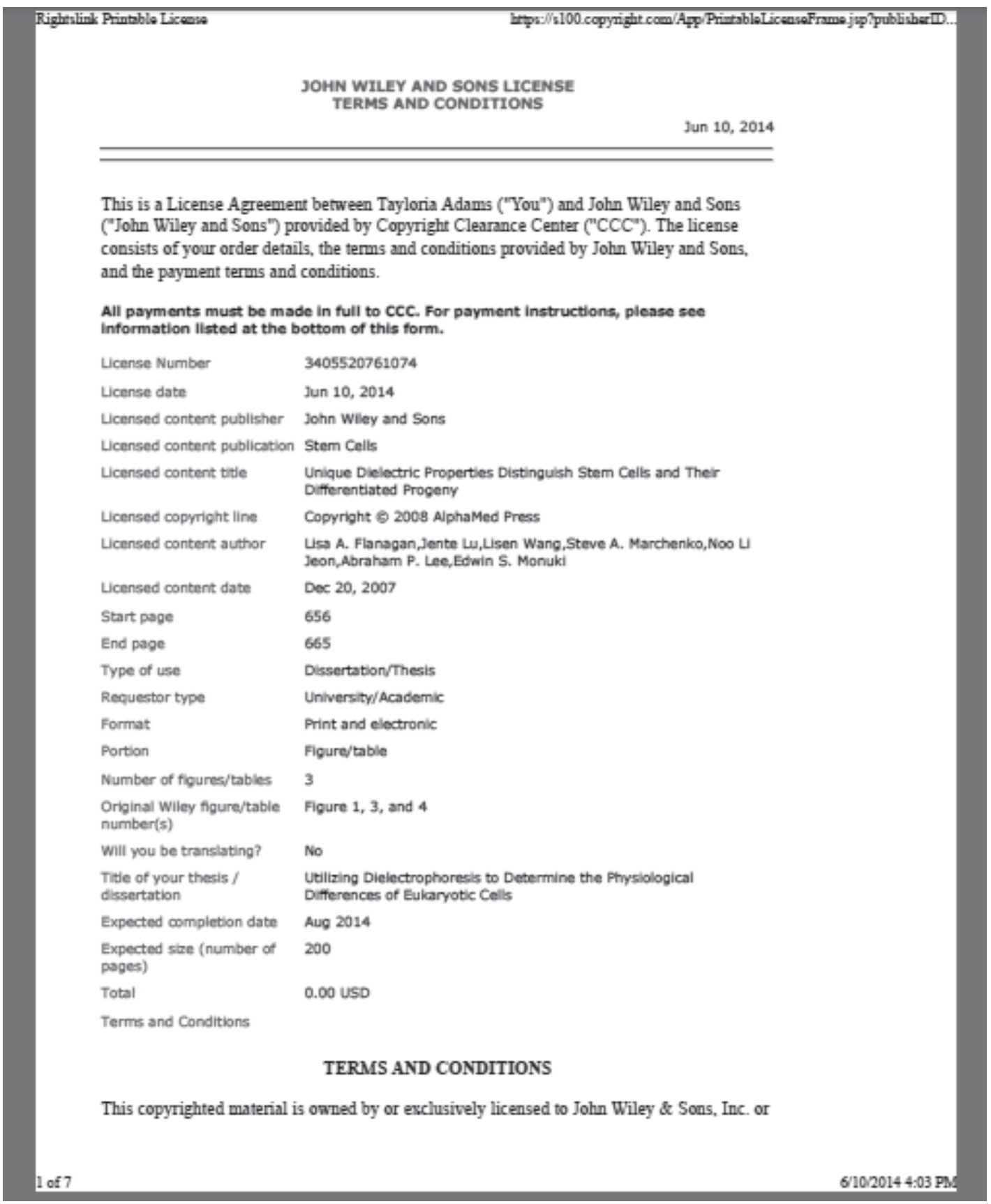


one of its group companies (each a"Wiley Company") or handled on behalf of a society with which a Wiley Company has exclusive publishing rights in relation to a particular work (collectively "WILEY"). By clicking $\uparrow$ accept $\$$ in connection with completing this licensing transaction, you agree that the following terms and conditions apply to this transaction (along with the billing and payment terms and conditions established by the Copyright Clearance Center Inc., ("CCC's Billing and Payment terms and conditions"), at the time that you opened your Rightslink account (these are available at any time at hittp://mraccount.copyright.com)

\section{Terms and Conditions}

- The materials you have requested permission to reproduce or reuse (the "Wiley Materials") are protected by copyright.

- You are hereby granted a personal, non-exclusive, non-sub licensable (on a stand-alone basis), non-transferable, worldwide, limited license to reproduce the Whey Materials for the purpose specified in the licensing process. This license is for a one-time use only and limited to any maximum distribution number specified in the license. The first instance of republication or reuse granted by this licence must be completed within two years of the date of the grant of this licence (although copies prepared before the end date may be distributed thereafter). The Wiley Materials shall not be used in any other manner or for any other purpose, beyond what is granted in the license. Permission is granted subject to an appropriate acknowledgement given to the author, title of the material/book/journal and the publisher. You shall also duplicate the copyright notice that appears in the Wiley publication in your use of the Wiley Material Permission is also granted on the understanding that nowhere in the text is a previously published source acknowledged for all or part of this Wiley Material. Any third party content is expressly excluded from this permission.

- With respect to the Wiley Materials, all rights are reserved. Except as expressly granted by the terms of the license, no part of the Wiley Materials may be copied. modified, adapted (except for minor reformatting required by the new Publication), translated, reproduced, transferred or distributed, in any form or by any means, and no derivative works may be made based on the Wiley Materials without the prior permission of the respective copyright owner. You may not alter, remsove or suppress in any manner any copyright, trademark or other notices displayed by the Wiley Materials. You may not license, rent, sell, loan, lease, pledge, offer as security, transfer or assign the Wiley Materials on a stand-alone basis, or any of the rights granted to you hereunder to any other person.

- The Wiley Materials and all of the intellectual property rights therein shall at all times remain the exclusive property of John Wiley \& Sons Inc, the Wiley Companies, or their respective licensors, and your interest therein is only that of having possession of and the right to reproduce the Wiley Materials pursuant to Section 2 herein during the continuance of this Agreement. You agree that you own no right, title or interest in or to the Wiley Materials or any of the intellectual property rights therein. You shall have 


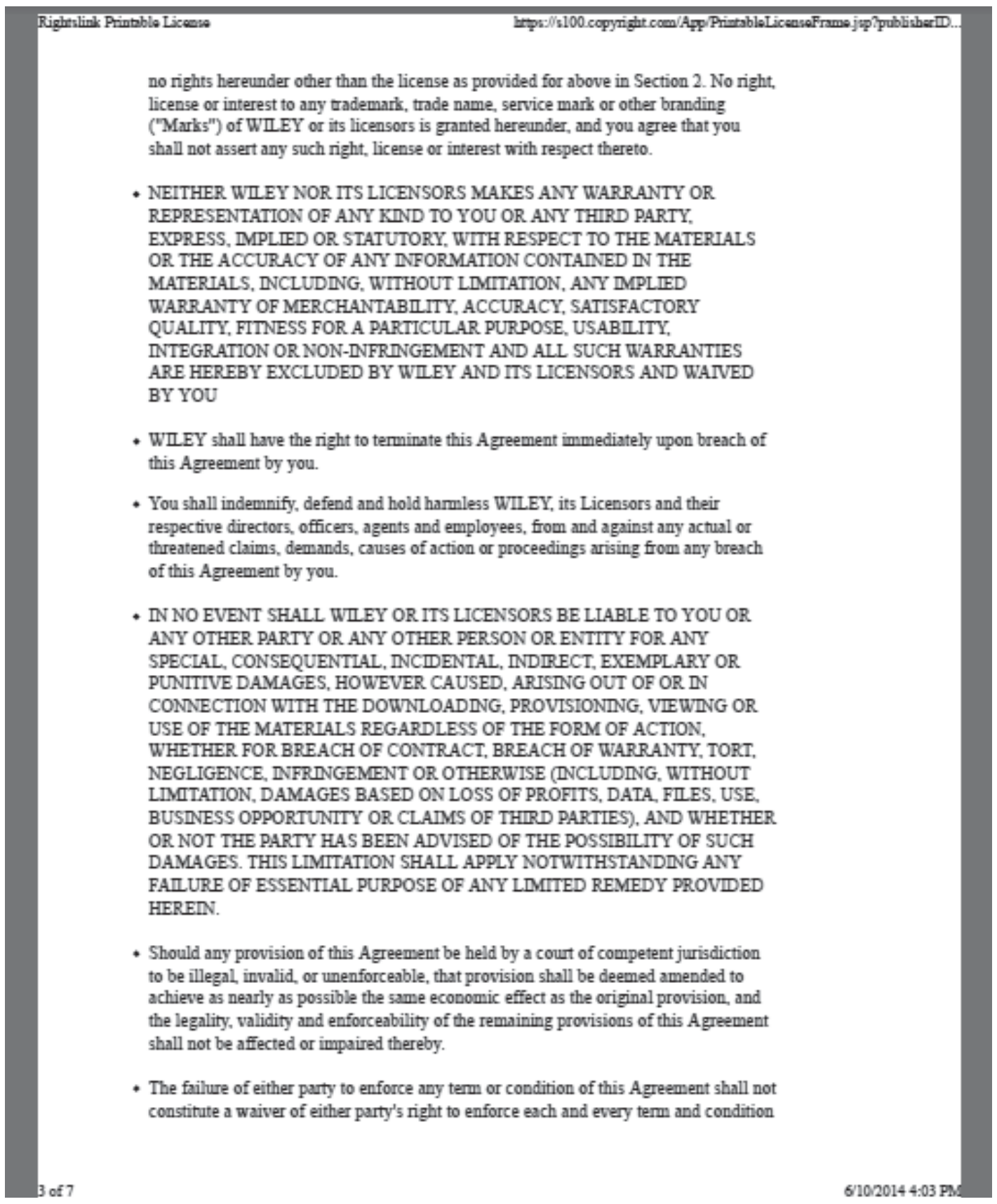




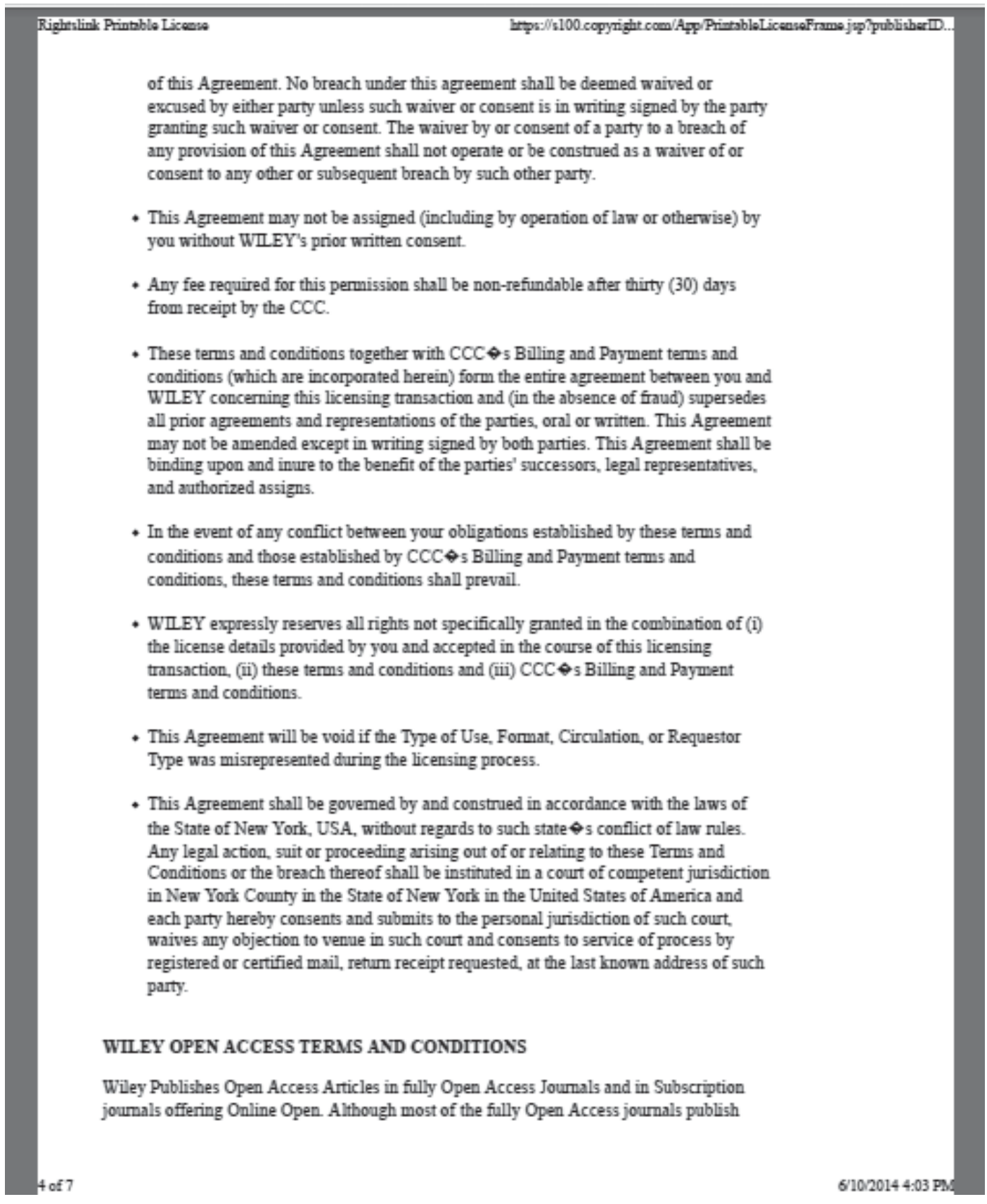


open access articles under the terms of the Creative Commons Attribution (CC BY) License only, the subscription joumals and a few of the Open Access Joumals offer a choice of Creative Commons Licenses:: Creative Commons Attribution (CC-BY) license Creative Commons Attribution Non-Commercial (CC-BY-NC) license and Creative Commons Attribution Non-Commercial-NoDerivs (CC-BY-NC-ND) License. The license type is clearly identified on the article.

Copyright in any research article in a joumal published as Open Access under a Creative Commons License is retained by the author(s). Authors grant Wiley a license to publish the article and identify itself as the original publisher Authors also grant any third party the right to use the article freely as long as its integrity is maintained and its original authors, citation details and publisher are identified as follows: [Title of Article/Author/Journal Title and Volume/Issue. Copyright (c) [year] [copyright owner as specified in the Joumal]. Links to the final article on Wiley $\vartheta_{5}$ website are encouraged where applicable.

\section{The Creative Commons Attribution License}

The Creative Commons Attribution License (CC-BY) allows users to copy, distribute and transmit an article, adapt the article and malse commercial use of the article. The CC-BY license permits commercial and non-commercial re-use of an open access article, as long as the author is properly attributed.

The Creative Commons Attribution License does not affect the moral rights of authors, including without limitation the right not to have their work subjected to derogatory treatment. It also does not affect any other rights held by authors or third parties in the article, including without limitation the rights of privacy and publicity. Use of the article must not assert or imply, whether implicitly or explicitly, any connection with, endorsement or sponsorship of such use by the author, publisher or any other party associated with the article.

For any reuse or distribution, users must include the copyright notice and make clear to others that the article is made available under a Creative Commons Attribution license, linking to the relevant Creative Commons web page.

To the fullest extent permitted by applicable law, the article is made available as is and without representation or warranties of any kind whether express, implied, statutory or otherwise and inchding, without limitation, wananties of title, merchantability, fitness for a particular purpose, non-infringement, absence of defects, accuracy, or the presence or absence of enrors.

\section{Creative Commons Attribution Non-Commercial License}

The Creative Commons Attribution Non-Commercial (CC-BY-NC) License permits use, distribution and reproduction in any medium, provided the original work is properly cited and is not used for commercial purposes.(see below)

\section{Creative Commons Attribution-Non-Commercial-NoDerivs License}

The Creative Commons Attribution Non-Commercial-NoDerivs License (CC-BY-NC-ND) 


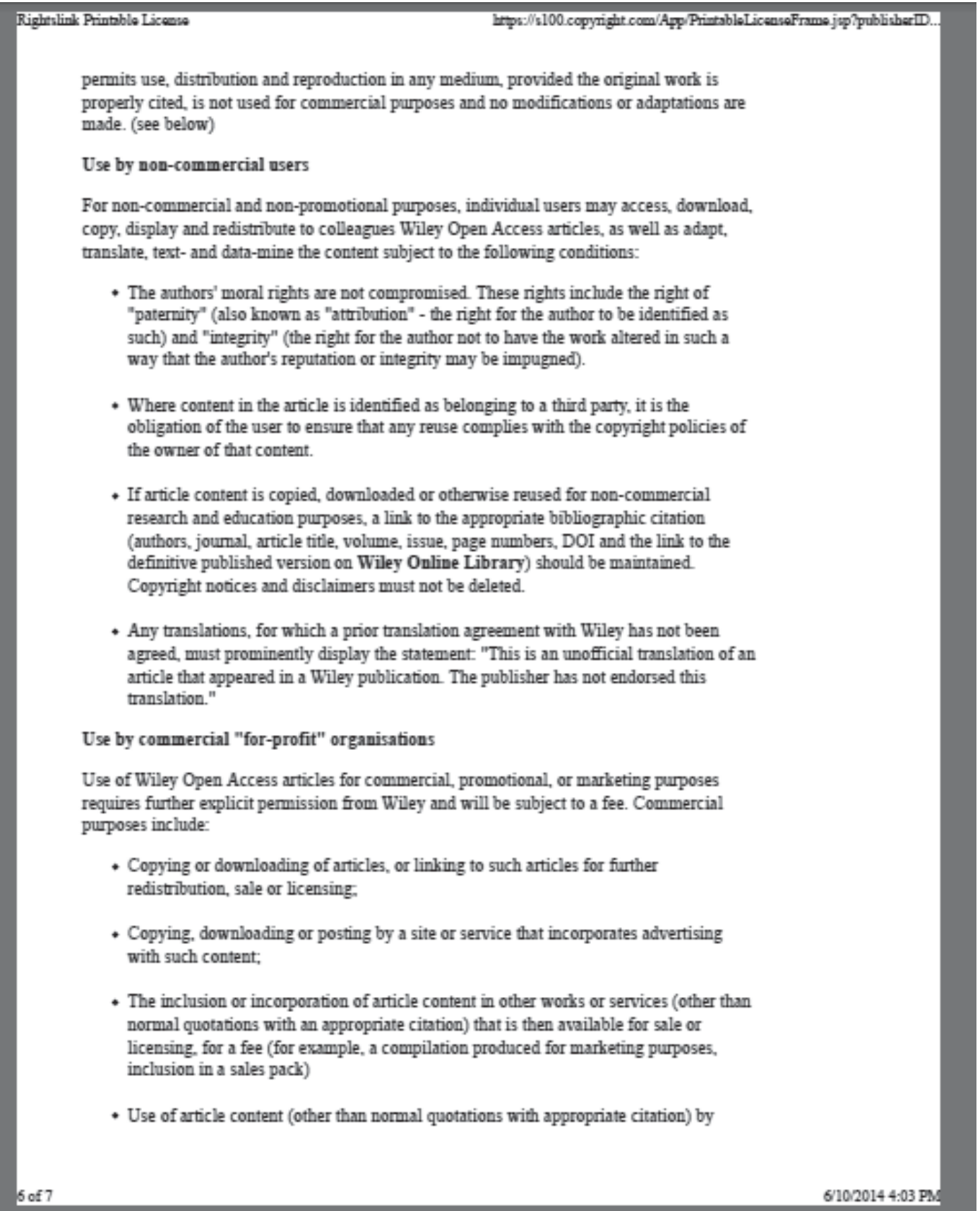




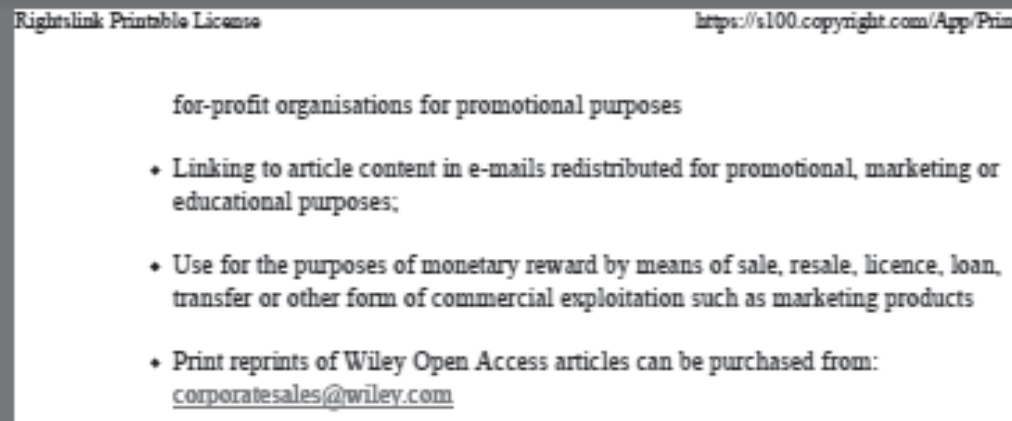

- Linking to article content in e-mails redistributed for promotional, marketing or educational purposes;

- Use for the purposes of monetary reward by means of sale, resale, licence, loan, transfer or other form of commercial exploitation such as marketing products

- Print reprints of Wiley Open Access articles can be purchased from: corporatesales@wiley.com

Further details can be found on Wiley Online Library hitp///olabout.wiley.com/WileyCDA /Section/id-410895.html

Other Terms and Conditions:

v1.9

If you would like to pay for this license now, please remit this license along with your payment made payable to "COPYRIGHT CLEARANCE CENTER" otherwise you will be involced within $\mathbf{4 8}$ hours of the license date. Payment should be in the form of a check or money order referencing your account number and this invoice number 501324869. Once you receive your involce for this order, you may pay your involce by credit card.

Please follow instructions provided at that time.

Make Payment To:

Copyright Clearance Center

Dept 001

P.O. Box 843006

Boston, MA 02284-3006

For suggestions or comments regarding this order, contact RightsLink Customer Support! customercare copyright:com or +1-877-622-5543 (toll free in the US) or +1-978-646-2777.

Gratis licenses (referending $\$ 0$ in the Total fleid) are free. Please retain this printable license for your reference. No payment is required. 


\section{B.12 Chapter 4 Permission}

Dottalink Mintabl Lemu

\section{AIP PUBLISHING LLC LICENSE TERMS AND CONDITIONS}

Dec 04, 2014

All payments must be made in full to CCC. For payment instructions, please see information listed at the bottom of this form.

License Number

Order Date

Publisher

Publication

Article Title

Author

Online Publication Date

Volume number

Issue number

Type of Use

Requestor type

Format

Portion

Will you be translating?

Title of your thesis /

dissertation

Expected completion date

Estimated size (number of pages)

Total

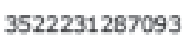

Dec 04, 2014

AIP Publishing ULC

Biomicrofluidics

Characterizing the dielectric properties of human mesenchymal stem cells and the effects of charged elastin-like polypeptide copolymer treatment

T. N. G. Adams,P. A. Tumer,A. V. Janorkar, et al.

$\operatorname{Sep} 16,2014$

8

5

Thesis/Dissertation

Author (original article)

Print and electronic

Excerpt (> 800 words)

No

UTILIZING DIELECTROPHORESIS TO DETERMINE THE PHYSIOLOGICAL DIFFERENCES OF EUKARYOTIC CELLS

Dec 2014

250

0.00 USD

Terms and Conditions

AIP Publishing $\amalg C$ - Terms and Conditions: Permissions Uses

AIP Publishing LC ("AIPP") hereby grants to you the non-exclusive right and license to use and/or distribute the Material according to the use specified in your order, on a one-time basis, for the specifled term, with a maximum distribution equal to the number that you have ordered. Any links or other content accompanying the Material are not the subject of this license.

1. You agree to include the following copyright and permission notice with the reproduction of the Material:"Reprinted with permission from [FULL CITATION]. Copyright [PUBLICATION YEAR], AIP Publishing LLC." For an article, the copyright and permission notice must be printed on the first page of the article or book chapter. For photographs, covers, or tables, the copyright and permission notice may appear with the Material, in a footnote, or in the reference list.

2. If you have licensed reuse of a figure, photograph, cover, or table, it is your responsibility to ensure that the material is original to AIPP and does not contain the copyright of another entity, and that the copyright notice of the figure, photograph, cover, or table does not 
indicate that it was reprinted by AIPP, with permission, from another source. Under no circumstances does AIPP, purport or intend to grant permission to reuse material to which it does not hold copyright.

3. You may not alter or modify the Material in any manner. You may transiate the Material into another language only if you have licensed translation rights. You may not use the Material for promotional purposes. AIPP reserves all rights not specifically granted herein.

4. The foregoing license shall not take effect unless and until AIPP or its agent, Copyright Clearance Center, receives the Payment in accordance with Copyright Clearance Center Billing and Payment Terms and Conditions, which are incorporated herein by reference.

5. AIPP or the Copyright Clearance Center may, within two business days of granting this license, revoke the license for any reason whatsoever, with a full refund payable to you. Should you violate the terms of this license at any time, AIPP, AIP Publishing LLC, or Copyright Clearance Center may revoke the license with no refund to you. Notice of such revocation will be made using the contact information provided by you. Failure to receive such notice will not nullify the revocation.

6. AIPP makes no representations or warranties with respect to the Material, You agree to indemnify and hold harmless AIPP, AIP Publishing UC, and their officers, directors, employees or agents from and against any and all claims arising out of your use of the Material other than as specifically authorized herein.

7. The permission granted herein is personal to you and is not transferable or assignable without the prior written permission of AIPP. This license may not be amended except in a writing signed by the party to be charged.

8. If purchase orders, acknowledgments or check endorsements are lissued on any forms containing terms and conditions which are inconsistent with these provisions, such inconsistent terms and conditions shall be of no force and effect. This document, includin? the CCC Billing and Payment Terms and Conditions, shall be the entire agreement between the parties relating to the subject matter hereof.

This Agreement shall be governed by and construed in accordance with the laws of the State of New York. Both parties bereby submit to the jurisdiction of the courts of New York County for purposes of resolving any disputes that may arise hereunder.

Questions? customercarefecopyright-com or +1-855-239-3415 (toll free in the US) or +1-978-646-2777.

Gratis licenses (referencing $\mathbf{\$ 0}$ in the Total fleld) are free. Please retain this printable license for your reference. No payment is required. 


\section{B.13 Chapter 5 Permission}

Moghtilink Mintable Lewne

MION14, 6:12 MM

\section{AIP PUBLISHING LC LICENSE TERMS AND CONDITIONS}

All payments must be made in full to CCC. For payment instructions, please see information listed at the bottom of this form.

License Number 3445570572494

Order Date

Aug 10, 2014

Publlsher

AIP Publishing $u C$

Publication

Biomicrofluidics

Article Tnile

Frequency sweep rate dependence on the dielectrophoretic response of polystyrene beads and red blood cells

Author

Online Publication Date

T. N. G. Adams, K. M. Leonard,A. R. Minerick

Volume number

Dec 10, 2013

Issue number

7

Type of Use

6

Requestor type

Thesis/Dissertation

Format

Portion

Will you be translating?

Title of your thesis /

dissertation

Author (original article)

Print and electronic

Excerpt ( $>800$ words)

No

Utilizing Dielectrophoresis to Determine the Physiological Differences of Eukaryotic Cells

Expected completion date Aug 2014

Estimated size (number of 200

pages)

Total

0.00 USD

Terms and Conditions

AIP Publishing LLC -- Terms and Conditions: Permissions Uses

AIP Publishing LLC ("AIPP") hereby grants to you the non-ewdusive right and license to use and/or distribute the Material according to the use specified in your order, on a one-time basis, for the specified term, with a maximum distribution equal to the number that you have ordered. Any liniks or other content accompanying the Material are not the subject of this license.

1. You agree to include the following copyright and permission notice with the reproduction of the Material:"Reprinted with permission from [FULL CITATION]. Copyright [PUBLICATION YEAR], AIP Publishing UC. For an article, the copyright and permission notice must be printed on the first page of the article or book chapter. For photographs, covers, or tables, the copyright and permission notice may appear with the Material, in a footnote, or in the reference list.

2. If you have licensed reuse of a figure, photograph, cover, or table, it is your responsibility to ensure that the material is original to AIPP and does not contain the copyright of another entity, and that the copyright notice of the figure, photograph, cover, or table does not indicate that it was reprinted by AIPP, with permission, from another source. Under no 
circumstances does AIPP, purport or intend to grant permission to reuse material to which it does not hold copyright.

3. You may not alter or modify the Material in any manner. You may translate the Material into another language only if you have licensed translation rights. You may not use the Material for promotional purposes. AIPP reserves all rights not specifically granted herein.

4. The foregoing license shall not take effect unless and until AIPP or its agent, Copyright Clearance Center, receives the Payment in accordance with Copyright Clearance Center Billing and Payment Terms and Conditions, which are incorporated herein by reference.

5. AIPP or the Copyright Clearance Center may, within two business days of granting this license, revoke the license for any reason whatsoever, with a full refund payable to you. Should you violate the terms of this license at any time, AIPP, AIP Publlshing LLC, or Copyright Clearance Center may revolke the license with no refund to you. Notice of such revocation will be made using the contact information provided by you. Failure to recelve such notice will not nullify the revocation.

6. AIPP makes no representations or warranties with respect to the Material. You agree to indemnify and hoid harmless AIPP, AIP Publishing LLC, and their officers, directors, employees or agents from and against any and all daims arising out of your use of the Material other than as specifically authorized herein.

7. The permission granted herein is personal to you and is not transferable or assignable without the prior written permission of AIPP. This license may not be amended except in a writing signed by the party to be charged.

8. If purchase orders, acknowledgments or check endorsements are issued on any forms containing terms and conditions which are inconsistent with these provisions, such inconsistent terms and conditions shall be of no force and effect. This document, induding the $C C C$ Billing and Payment Terms and Conditions, shall be the entire agreement between the parties relating to the subject matter hereof.

This Agreement shall be governed by and construed in accordance with the laws of the State of New York. Both parties bereby submit to the jurisdiction of the courts of New York County for purposes of resolving any disputes that may arise bereunder.

You will be involced within $\mathbf{4 8}$ hours of this transaction date. You may pay your invoice by credit card upon receipt of the invoice for this transaction. Please follow instructions provided at that time.

To pay for this transaction now; please remit a copy of this document along with your payment. Payment should be in the form of a check or money order referencing your account number and this invoice number RLNKS01373866.

Make payments to "COPYRIGHT CLEARANCE CENTER" and send to:

\section{Copyright Clearance Center}

Dept 001

P.O. Box 843006

Boston, MA 02284-3006

Please disregard electronic and mailed copies if you remit payment in advance.

Questions? customercarefecorriahthcom or +1-855-239-3415 (toll free in the US) or +1-978-646-2777.

Gratis licenses (referencing $\$ 0$ in the Total field) are free. Please retain this printable license for your reference. No payment is required. 


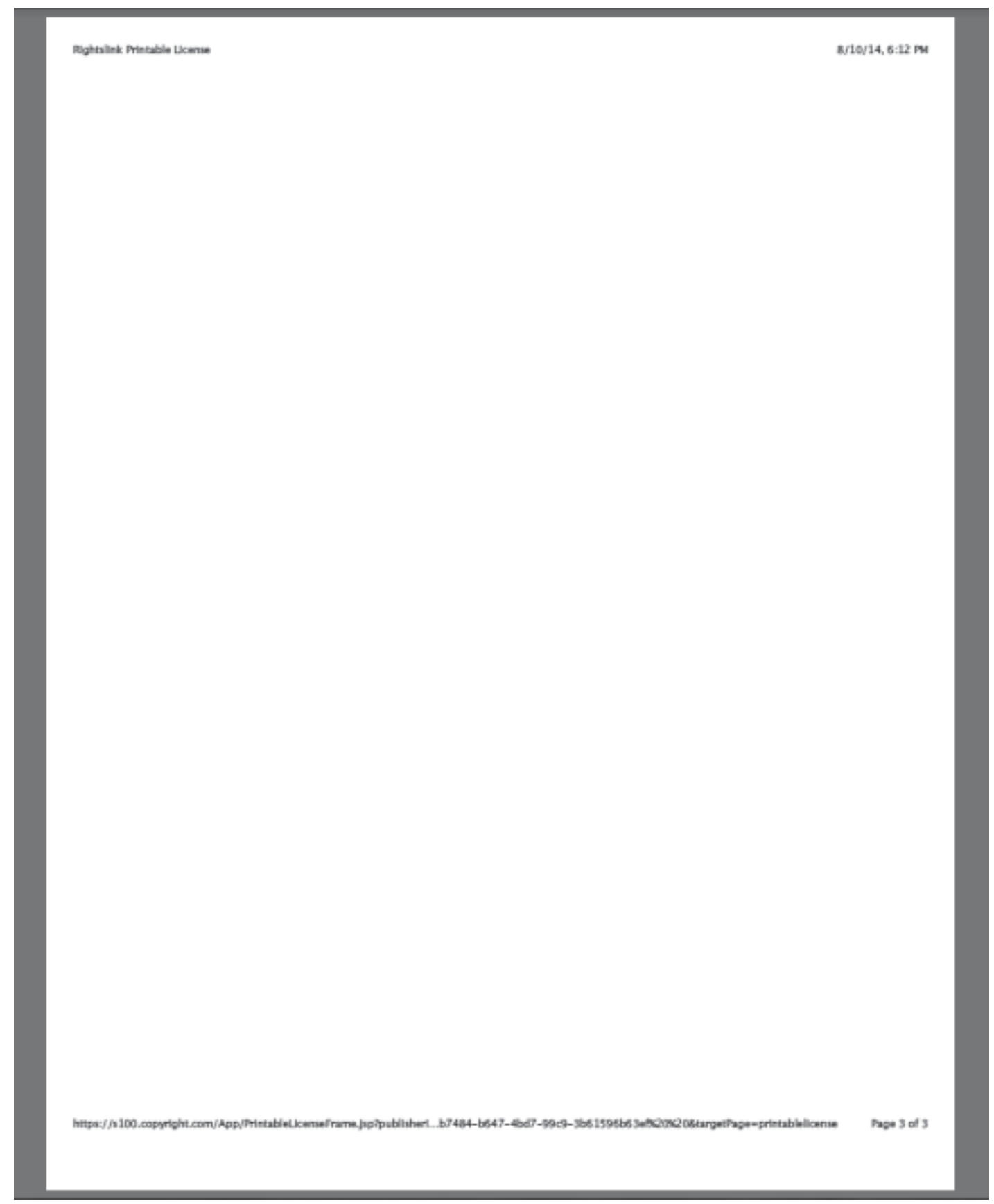




\section{B.14 Appendix A Permission}

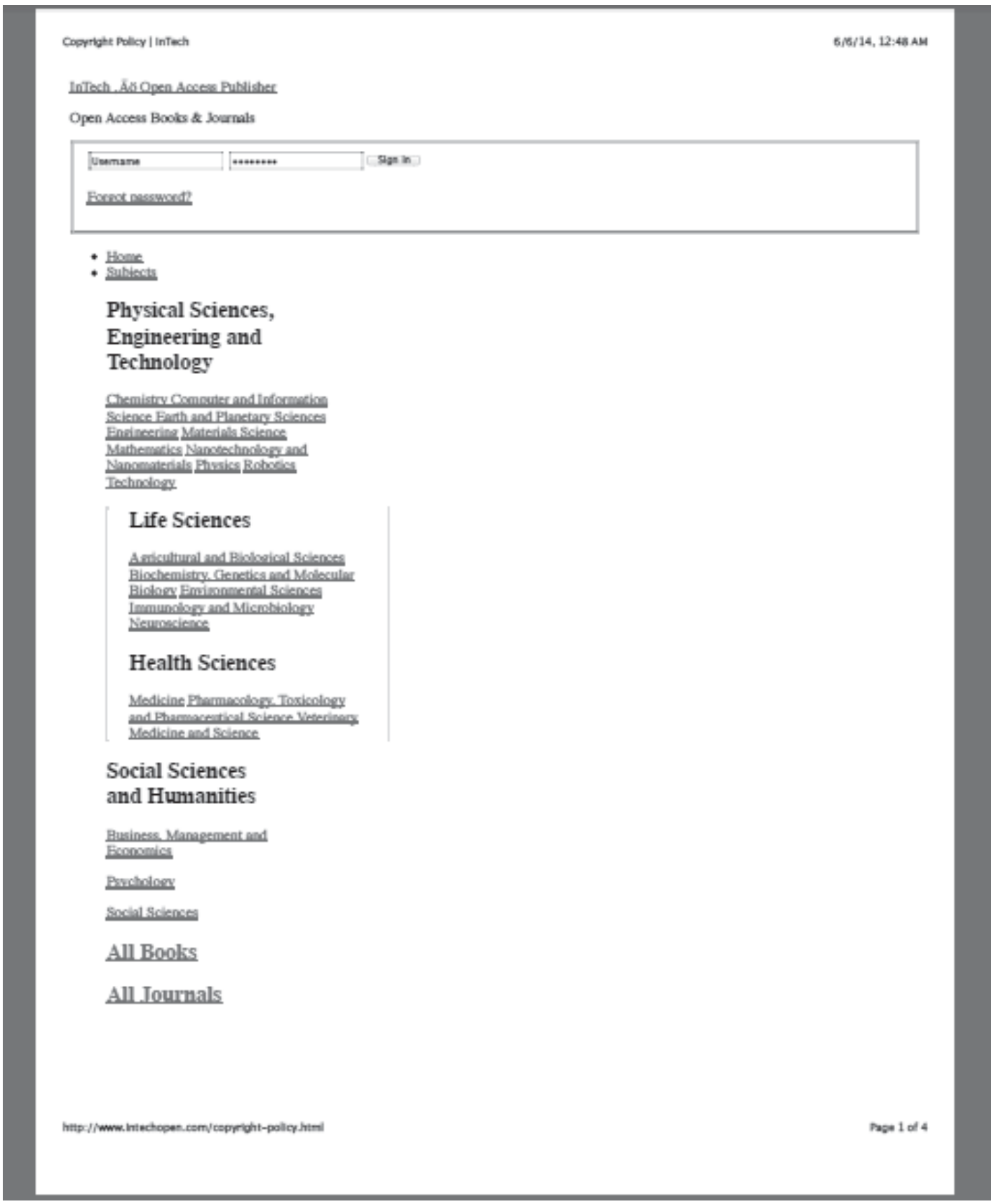


Read, Share and

Download for FREE

* OarAuthos

- Open Access

- Atbout intreal

Searth putlication.

Bublishwith InTech

\section{Copyright Policy}

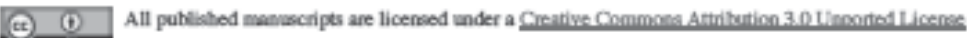

Our Copgright Folicy aims to guanantee that original material is peblished while at the same time giving significant freedom to our wathors. InTech upbolds a very flevible copyright policy meaning that there is mo copyright tmanter to the publisher and wuthors hold exclusive copyright to their work

When submirting their manuscripes auboes are required to accept the terms and conditions set forth in our Copyrighe Agreement as follows:

\section{Author's Retentice of Rights}

InTech ackaowlodges and agrees that the Author(s) retain(s) the copyright to the work submittod for publication, and islare allowedt

1. to revise, reprodice, distribute, publicly perform, and publicly display the ChapteviArticle

2 to republish the Work, as loeg as InTech is citod as the source of fist poblication of the Work

3. to prepere derivative works from the Cometer/Arsele

4 to apply all other proprietary rights to the Wod (such as pontents), and

$s$ to authorize ochers to make any use of the Chapteri'Article.

Users are granted the right to copy, use, distribute, transmit and display the Work publicly, and to create and distribute derivative works in any medium and for any respoesible purpose, as long as the Author receives credit as author, and the Bookijournal in which the Chagceri'Article has been published is citod las the source of fist publication of the Work:

\section{Permbsaben to use}

The author aed ges to InTech all rights:

1. to publish the Wodk, in whole or in pert, by various means such as hand copy or electronic copy formats, and in uny and all forms of media. now or hereafter kown; and

2 to republish, distribute, promote, publicly perform, and publicly display the Work at any time and in all InTech Publishing Grocep projects.

\section{Author's responsibillties}

InTech distritutes is publicabios throughout the wodd and wants to ensure that the material submitied to its publications is progerly avallable to the readership of those publications. Aurhoss must ensure that their Chapeer/Article meets the requiremense, inclading poovisbos covering originality, anthorship, author responsibilities and athor miscoeduct. Therefore:

1. the Aurhor(s) should caly submit original work that has neither appearod elsewhere for publication, nor is under review for anocher refereed publicason

2 the Aurbos(s) should detemine whether disclosure of their material, content and photographs requires the prior consene of ceter parties and, if so, should obecin it; and

3. revearch carnied out in collaborasion with other scholars necessitutes that all aurhors approve of submining the ChapteriArticle. 


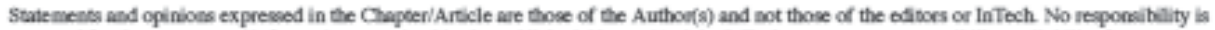
acceped for the accuracy of information consained in the peblished Chapterifarticle. InTech assumes no respoesibility or liablity for any damage

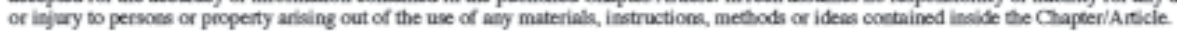

\section{General terms}

By acoepting the Copyright Agreement the cosesponding Author:

1. warnants that heishe is the Aurhor and hea the power and athority to malo and execure this assignmene

2 aftirms thac, for jointly authored Chaptend Articles, heishe is empoweved to accept this form on behalf of all authors

3. warmants that the ChapteríArticle is original, has not previously been pablished and is soc currently under coesideration for publication by any other enciky

4. affirma that permission will be obtainod for all previously publishod andior copynighted material contained in this manuscript (to the exient that the chapter inocorporites text, passages, figures, disa or other muserial from the work of others), it is the authors' collective responsibility to obceain all copyright permibebions - they can be obtained during the publibhing process and need to be collectod before publication and

5. agrees to indemnify lif fech against all bosses, costs and expenses (including legal ooss and expences) arising from claims made by other

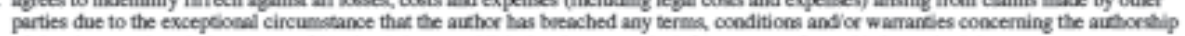
of the chapter (the whole or parts of in), the rights to poblish the chagter or the infringement of any thind party's rights.

If the Aubor has prepared the work as part of that hisher offlcial duties as an employee of officer of a Government anency, public instizion of company and has copyrighes that belong to the Governmens agency. public institution, or oompeny. InTech agrees to accept their terms and conditions if they are nok in coeflict widh InTech's Copyright Folicy.

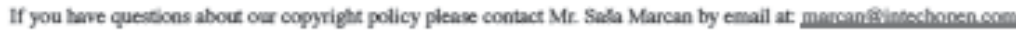

\section{Treostones}

\section{About us}

- About inTech

- Scientific Boond

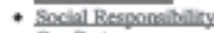

- Dor Priners

* DohisitinTeal

* Coninact

Open Aceess

* Alocut Dien Acaresa

+ How it Whats

- Benefisis of Coven Acocese

- InTech and Open Accex

* Eoclingaries

- OALPMH

\section{Participate}

- Puklish with InTech

- InTechls Publishing Principlea

* Start Your Oern Poon

+ Aufhorí Actmouledgments

+ EAOS

Policies

+ Priwav Pollicy

- Copyrigir Policy

* Authorshis Policas

- Peer Review Policy

- Prior Pukliontion Folicy 


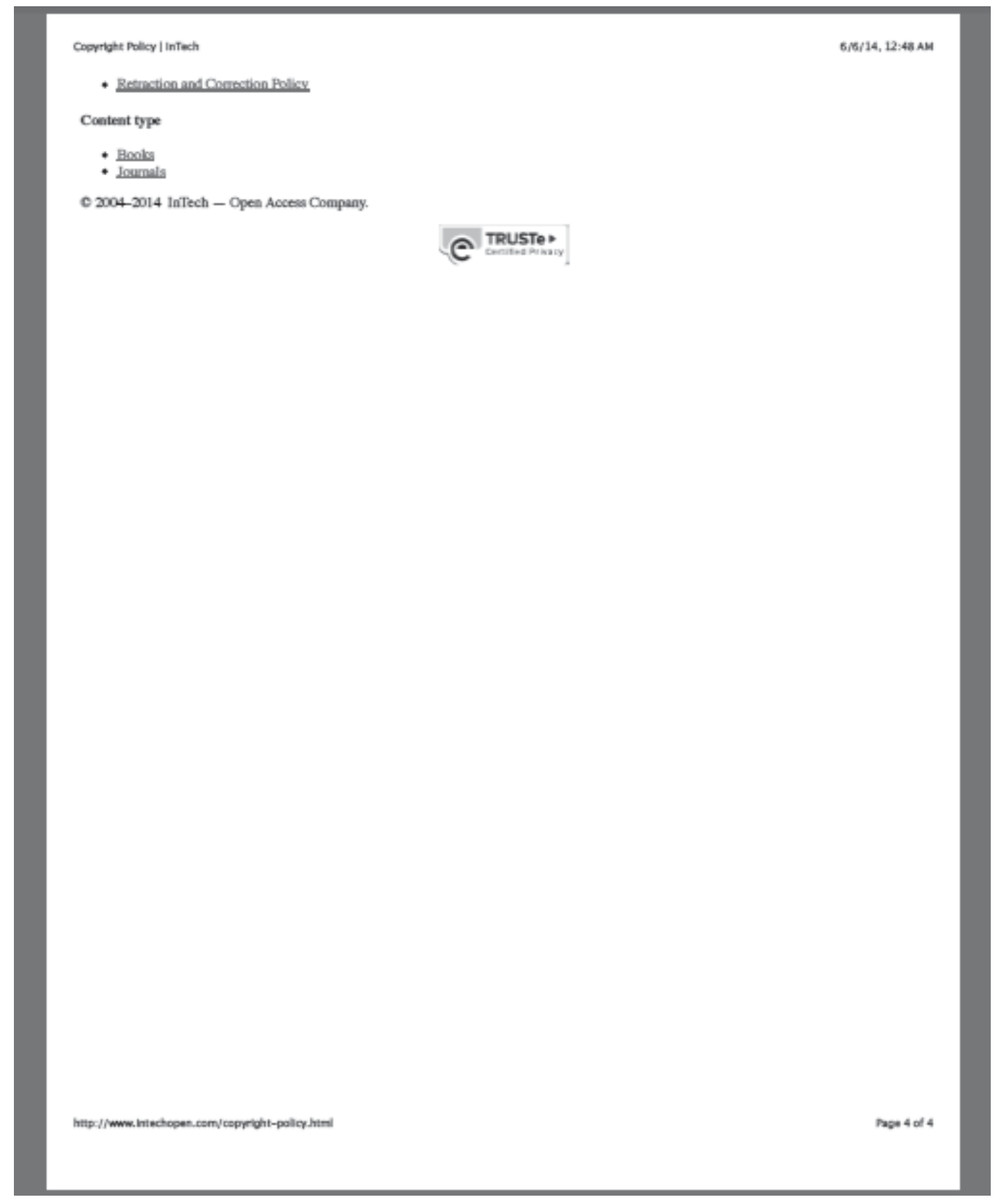

